\title{
Improved Arterial Input Function For Dynamic Contrast-Enhanced Magnetic Resonance Imaging Using Phase and $\mathrm{T}_{1}$ Measurements
}

by

\section{Nicholas Majtenyi}

A thesis submitted to the Faculty of Graduate and Postdoctoral Affairs

in partial fulfillment of the requirements for the degree of

Doctor of Philosophy

in

Physics

Specialization in Medical Physics

Ottawa-Carleton Institute for Physics

Carleton University

Ottawa, Ontario

(C)2019

Nicholas Majtenyi 


\section{Abstract}

Perfusion is a bodily function that describes the transport of blood and nutrients to an organ. If characteristics of the blood flow to an organ can be measured, clinically useful quantitative data about the organ can be obtained. This is particularly useful in the diagnosis of tumours as their rapid and poorly-formed capillary network is unique compared to healthy tissue. Tumour perfusion information can be obtained by performing Dynamic Contrast-Enhanced (DCE)-MRI. Using DCE-MRI, blood flow into a tumour from a nearby artery can be computed by measuring the concentration of an injected contrast agent as a function of time. This measurement, known as the arterial input function (AIF), can be computed based on the change of either the intensity or the phase of the MR signal from the blood, due to the presence of a contrast agent. While both methods can be used to acquire the AIF, the change in phase is preferred due to its superior accuracy. Even so, the method conventionally used to obtain the phase-derived AIF has deficiencies that lead to an AIF intensity overestimation.

The goal of this thesis was to determine a better method to obtain AIF data for patients with brain tumours by using a combination of MR signal phase and accurate $T_{1}$ relaxation measurements. This was done by first characterizing and deriving equations for phase measurement errors and simulating these effects on the AIF using realistic, clinical parameters. $T_{1}$ measurement methodology was developed and validated in static- 
water phantoms and applied to a flowing-water phantom system on which phase AIF data were also acquired. The novel AIF method was tested on this system before it was applied clinically to patients diagnosed with high-grade gliomas. The differences between the quantitative perfusion parameters from the new method and previous AIF methods were compared.

The novel AIF measurement method presented in this thesis was designed to be less prone to experimental error than current clinical methods. Theoretical predictions, computed simulations, experimental work with MRI test objects, and clinical results all show that the new method for measuring the AIF is significantly superior to procedures currently used clinically. 


\section{Acknowledgments}

Firstly, I would like to thank my supervisor, Dr. Ian Cameron, for his continued support and encouragement throughout my time as a graduate student. He never missed an opportunity to share his knowledge and passion for MRI research. I cannot express how appreciative I am for all of the time and care he has spent with me over the course of this work. His guidance has allowed me to grow into a well-rounded scientist and I could not have imagined having a better advisor and mentor for my Ph.D.

I would like to thank the researchers and medical professionals at The Ottawa Hospital who helped me during the course of my research. I would specifically like to thank Dr. Thanh Nguyen for establishing the framework for a significant portion of the work presented here. His many insights and thoughts on this project have proved to be invaluable. A special thanks goes to Dr. Gregory Otis Cron for his never-ending guidance. From his help with study development, to spending many long days at the scanner, to our love and passion for GCSC, Dr. Cron will never stop being a friend and mentor to me.

Last but not least, I would like to thank those close to me, especially my wife Laura. This thesis could not be possible without your continuous love and support, especially when I needed it the most. I'd also like to thank my siblings and parents who helped to develop my love of science from a young age. They have supported me endlessly and have always been my biggest cheerleaders not only for this work, but for my entire life. 


\section{Table of Contents}

Abstract

Acknowledgments $\quad$ iv

Table of Contents $\quad$ v

List of Tables $\quad$ ix

List of Illustrations $\quad$ xi

List of Acronyms and Symbols $\quad$ xvi

1 Introduction 1

1.1 Principal Contributions $\ldots \ldots \ldots \ldots \ldots \ldots \ldots \ldots \ldots \ldots \ldots \ldots \ldots \ldots \ldots \ldots \ldots \ldots \ldots$

2 Anatomy and MRI Background $\quad 5$

2.1 Biology of Gliomas. . . . . . . . $5 \ldots \ldots \ldots \ldots \ldots \ldots \ldots \ldots \ldots \ldots \ldots \ldots \ldots \ldots$

2.1 .1 Glioma Formation $\ldots \ldots \ldots \ldots \ldots \ldots \ldots \ldots \ldots \ldots \ldots \ldots \ldots \ldots \ldots$

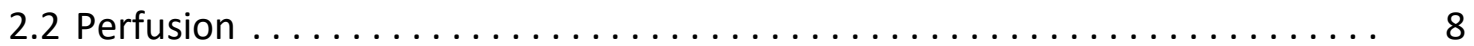

2.2.1 Tumour Vasculature Properties $\ldots \ldots \ldots \ldots \ldots \ldots \ldots \ldots \ldots$

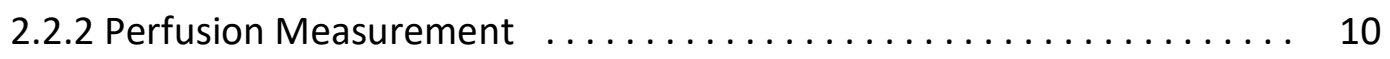

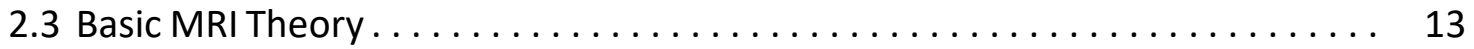

2.3.1 Magnetic Moments . . . . . . . . . . . . . . . . . . . 13

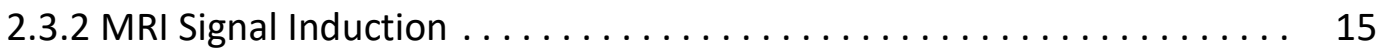

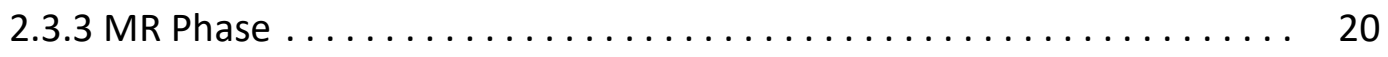

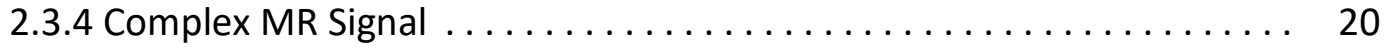

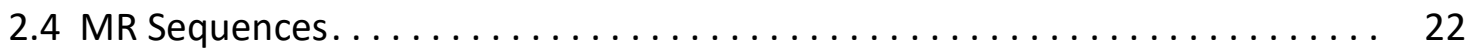

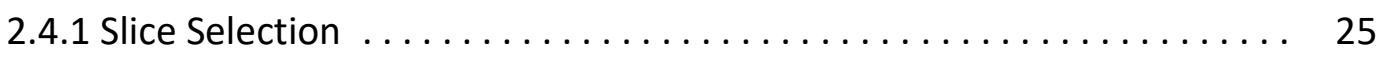

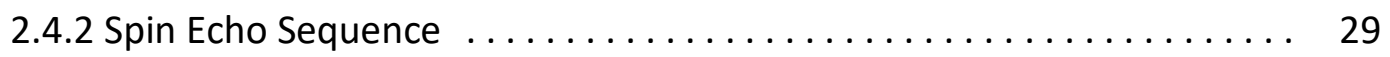


2.4.3 Gradient Echo Sequence $\ldots \ldots \ldots \ldots \ldots \ldots \ldots \ldots \ldots \ldots \ldots \ldots$

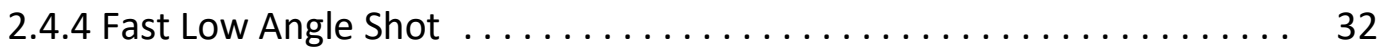

2.4 .5 Inversion Recovery $T_{1}$ Sequences $\ldots \ldots \ldots \ldots \ldots \ldots \ldots \ldots \ldots \ldots \ldots$

2.4.6 Look-Locker IR Sequences . . . . . . . . . . . . . . . . . 38

2.5 MRI Contrast Enhancement ......................... 40

2.5.1 Magnetic Susceptibility for an Infinite Cylinder . . . . . . . . . . . 43

2.5.2 Arterial Input Function $\ldots \ldots \ldots \ldots \ldots \ldots \ldots \ldots \ldots \ldots \ldots \ldots \ldots \ldots \ldots \ldots \ldots$

2.5.3 GBCA Effect on MR Signal Magnitude and Phase . . . . . . . . . . 47

3 Partial Volume Averaging and the Accuracy of the Arterial Input Function 51

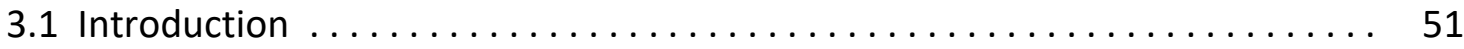

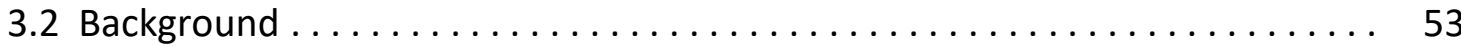

3.2.1 Comparison of Signal Intensity Versus Signal Phase for AIF Measurement. 53

3.2.2 Arterial Input Function Versus Venous Output Function . . . . . . . . . 55

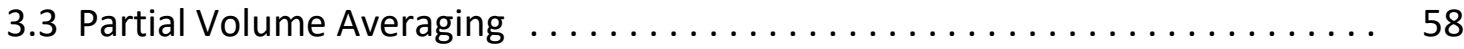

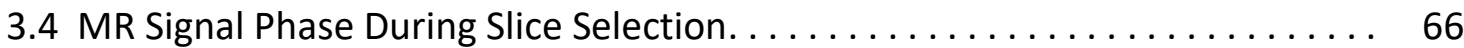

3.4.1 Phase Change Due to Magnetic Susceptibility . . . . . . . . . . . . . 67

3.4.2 Phase Change Due to Blood Flow ................. 70

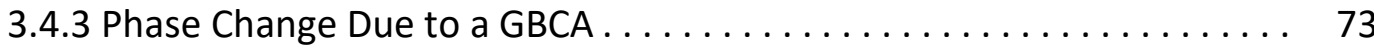

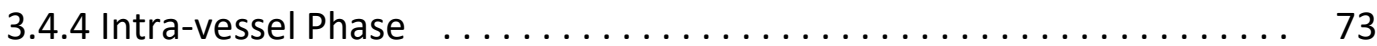

3.5 Phase Error Computations for Realistic Parameters . . . . . . . . . . . . . . . . 75

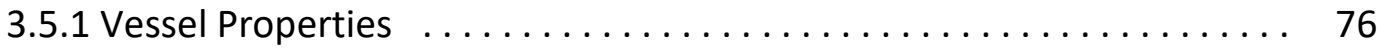

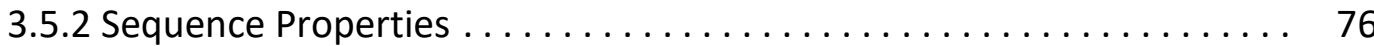

3.5.3 ROI and Vessel Models . . . . . . . . . . . . . . . . . . . . . . . . . . . . 77

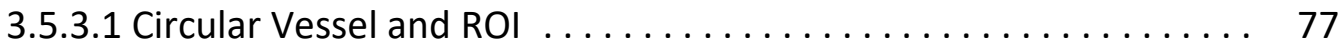

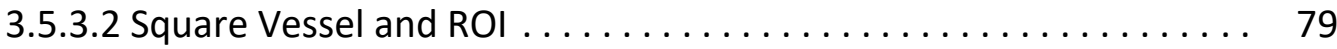

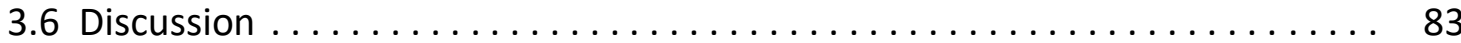

4 The Phase and $T_{1}$ Arterial Input Function 91

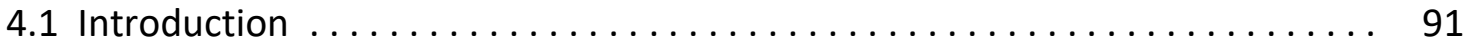

4.2 "Phase-Only" AlF and "Phase and $\mathrm{T}_{1}$ " AlF ................. 93

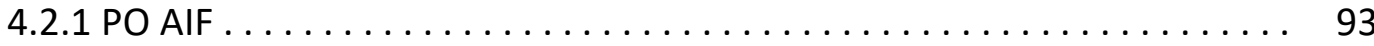

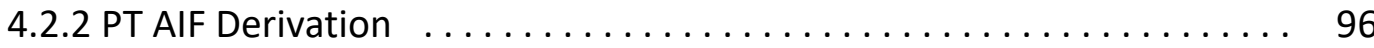


4.3 Simulation Methods . . . . . . . . . . . . . . . . . . . . . . 99

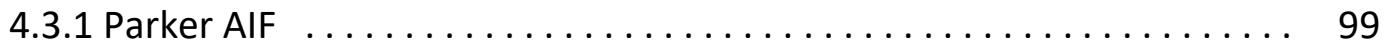

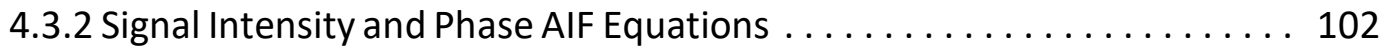

4.3.3 Simulated Noise . . . . . . . . . . . . . . . . . . . . . . . 102

4.3.4 Partial Volume Averaging . . . . . . . . . . . . . . . . . . . . . 105

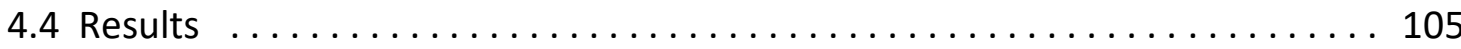

4.4.1 Simulated AlFs with Added Noise . . . . . . . . . . . . . . . . . . . . 105

4.4.2 The Effects of Partial Volume Averaging . . . . . . . . . . . . . . . . . . . 110

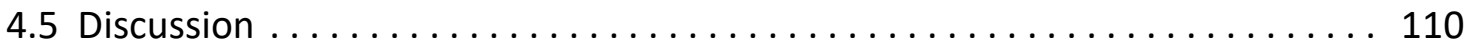

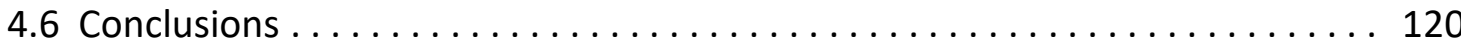

5 AIF Experiments Using Phantoms $\quad 121$

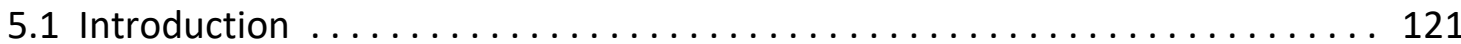

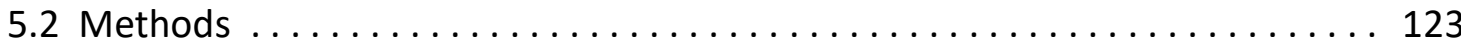

5.2.1 Phantom Design and Creation ....................... 123

5.2.1.1 Static Phantoms . . . . . . . . . . . . . . . . . . . . . . 123

5.2.1.2 Dynamic Phantom . . . . . . . . . . . . . . . . . . . 126

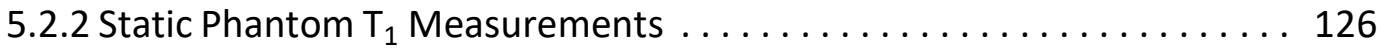

5.2.2.1 $\mathrm{T}_{1}$ Analysis Method. . . . . . . . . . . . . . . . . . . . . 130

5.2.3 Dynamic Phantom Measurements . . . . . . . . . . . . . . . . 132

5.3 Results . . . . . . . . . . . . . . . . . . . . . . . . . . . . . 137

5.3.1 Static Phantom Results . . . . . . . . . . . . . . . . . . . . . . . 137

5.3.2 Dynamic Phantom Results . . . . . . . . . . . . . . . . . . . . 141

5.4 Discussion ................................... 146

5.4.1 Static Phantom Data . . . . . . . . . . . . . . . . . . . . 147

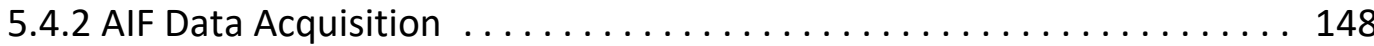

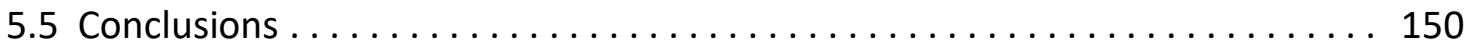

6 Clinical DCE-MRI Study 152

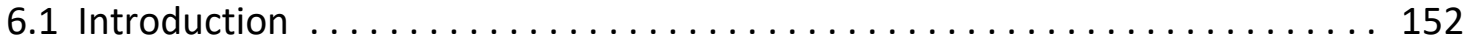

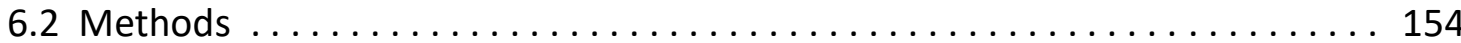

6.2.1 Study Design . . . . . . . . . . . . . . . . . . . . . . . . . . 154

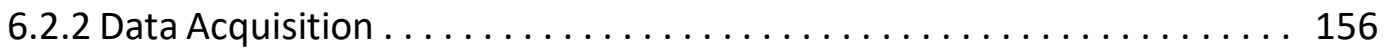




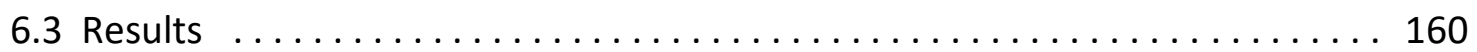

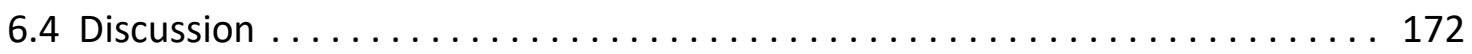

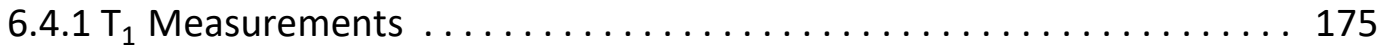

6.4.2 AlF Measurements ........................... 176

6.4.3 DCE-MRI Parameters . . . . . . . . . . . . . . . . . . . . . . . . . . . . . . . . 177

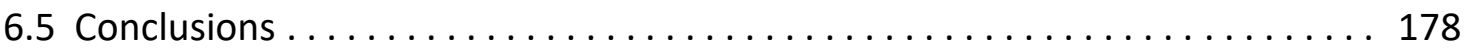

7 Conclusions 180

List of References 186 


\section{List of Tables}

3.1 Pixel fractions for the three circular vessels shown in Fig. $3.2 \ldots \ldots \ldots \ldots \ldots$

3.2 Summary of parameters used in $\Delta \phi_{\mathrm{PV}}$ calculations. The Hct value of 0.40 was used for females, 0.45 for males, and 0.431 as a weighted average

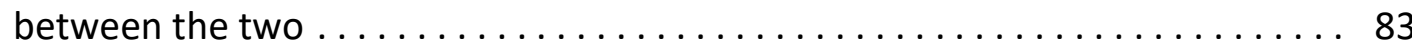

3.3 Phase error computation for $\mathrm{Hct}=0.45$ (males) and $\mathrm{F}=0 \ldots \ldots \ldots \ldots$

3.4 Phase error computation for $\mathrm{Hct}=0.40$ (females) and $\mathrm{F}=0 \ldots \ldots \ldots \ldots \ldots$

3.5 Phase error computation for $\mathrm{Hct}=0.431$ (study average) and $\mathrm{F}=0 \ldots \ldots 6$

3.6 Phase error computation for $\mathrm{Hct}=0.431$ (study average), $\mathrm{F}=3.7 \mathrm{~cm}^{3} \mathrm{~s}^{-1}$,

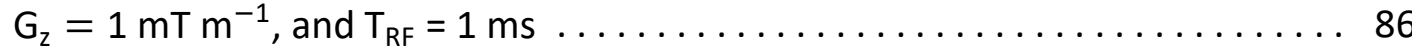

3.7 Phase error computation for $\mathrm{Hct}=0.431$ (study average), $\mathrm{F}=3.7 \mathrm{~cm}^{3} \mathrm{~s}^{-1}$,

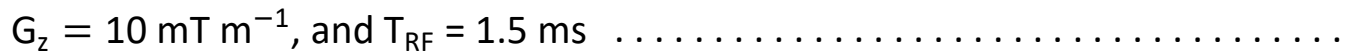

3.8 Phase error computation for $\mathrm{Hct}=0.431$ (study average), $\mathrm{F}=3.7 \mathrm{~cm}^{3} \mathrm{~s}^{-1}$,

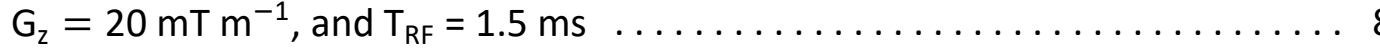

3.9 Summary of the baseline and washout phases $\left(\phi_{\mathrm{b}}\right.$ and $\phi_{\mathrm{w}^{\prime}}$, respectively) and the difference between the two for $\mathrm{f}=0.5, \mathrm{~F}=3.7 \mathrm{~cm}^{3} \mathrm{~s}^{-1}$, Hct $=0.431$

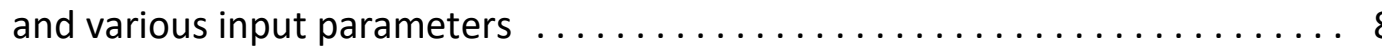

4.1 Parameters and standard deviation (SD) values used to create $\operatorname{AIF}_{\mathrm{p}}$ (Eq. 4.13), as given by Parker et al. [67]

4.2 Parameters used to compute the SI- and $\phi$-derived AlFs from the known AlF $F_{P}$ values. $M_{0}$ was chosen arbitrarily, while the values for $\alpha, T_{R}, T_{E}$, and $B_{0}$ were chosen based on the clinical DCE-MRI acquisition performed later in this work

5.1 GBCA volume $\left(V_{\mathrm{GBCA}}\right)$, water volume $\left(\mathrm{V}_{\mathrm{w}}\right)$, and their respective $[\mathrm{Gd}]$ with uncertainty for the 14 static phantoms 
5.2 Summary of $T_{1}$ and $T_{1}^{*}$ values and standard deviations from 12 static phantoms. Data are marked in terms of their statistical significant difference from the EPI-IR data using an unpaired $t$-test: $* * * P \leq 0.001$,

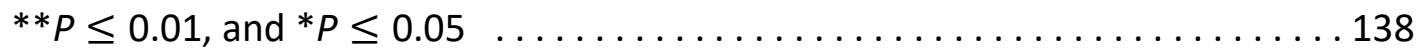

5.3 $r_{1}, r_{1}^{*}$, and Pearson correlation values computed for each $T_{1}$ method used $\ldots 138$

5.4 Dynamic phantom washout [Gd] values and standard deviations measured using the phase and $M O L L I_{\text {comb }} T_{1}$ methods. The values obtained from phase and $T_{1}$ are denoted $[\mathrm{Gd}]_{\phi}$ and $[\mathrm{Gd}]_{\mathrm{T}_{1}}$, respectively. The final column indicates the difference in the two previous columns $\ldots \ldots \ldots \ldots \ldots \ldots 145$

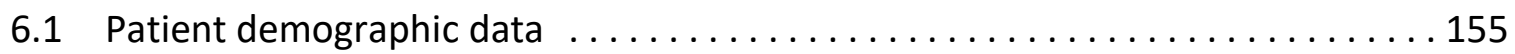

6.2 $\mathrm{MOLLI}_{0} \mathrm{~T}_{1,0}^{*}$ and $\mathrm{T}_{1,0}$ data measured in the SSS. The average \pm SD were obtained using error propagation for each patient. Note that patient 41 was not included in the analysis . . . . . . . . . . . . . . . . . 164

6.3 $\mathrm{MOLLI}_{0}$ and $\mathrm{MOLLI}_{1} \mathrm{~T}_{1, \mathrm{w}}^{*}$ and $\mathrm{T}_{1, \mathrm{w}}$ data measured in the SSS. The average \pm SD were obtained using error propagation for each patient. Note that patient 41 was not included in the analysis . . . . . . . . . . . . . . 165

6.4 $\mathrm{AlF}_{\mathrm{w}}^{*}$ and $\mathrm{AlF}_{\mathrm{w}}$ data and average $\pm \mathrm{SD}$ obtained using error propagation for each patient. Note that patient 41 was not included in the analysis . . . . . . 166

6.5 Difference in PT AIF ${ }_{w}^{*}$ and PT AIF ${ }_{w}$ values compared to the PO AIF value $_{w}$ for each patient. The average value does not include patient $41 \ldots \ldots \ldots$

6.6 DCE-MRI parameter values from the enhancing volumes for HGGs of grade III $(n=3)$, grade IV $(n=18)$, and grades III and IV together. The final column indicates the percent difference between the PO AIF values and (A) PT AlF ${ }_{\mathrm{w}}$ or (B) PT AIF ${ }_{\mathrm{w}}^{*}$ values. Data are listed as mean \pm standard deviation

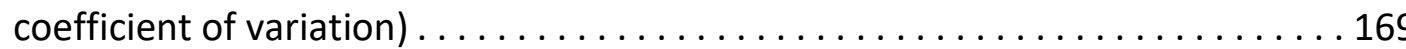




\section{List of Illustrations}

2.1 The comparison of two perfusion models. (A) The Kety model of perfusion includes a single-compartment where the GBCA moves from the vascular space into the EES. (B) For the extended Tofts-Kety model, the intravascular [Gd] is non-negligible and $v_{p}$ is included. In each image, the dotted background indicates what is included for each voxel $\ldots \ldots \ldots \ldots \ldots \ldots \ldots \ldots$

2.2 Motion of the magnetization after being tipped (A) from the longitudinal into the transverse plane. (B) The spins will dephase at different rates. (C) Eventually, the net transverse magnetization goes to zero $\ldots \ldots \ldots \ldots \ldots 21$

2.3 Schematic representation of the MR signal in the complex plane $\ldots \ldots \ldots \ldots 22$

2.4 A sequence diagram for a basic gradient echo MR sequence. Gradient pulses are shown as trapezoidal objects. The gradient pulses for the $\mathrm{Y}$-axis have different amplitudes each time the basic sequence block is repeated. $T_{R}$ and $\mathrm{T}_{\mathrm{E}}$ are the repetition time and echo time, respectively. The example shown

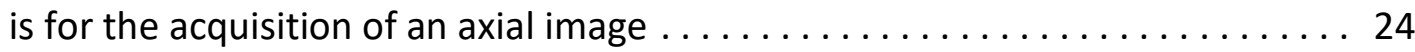

2.5 An example of the magnetization during a spin echo sequence in the $x^{\prime}-y^{\prime}$ plane. In this example of all the nuclear spins are slightly off resonance with

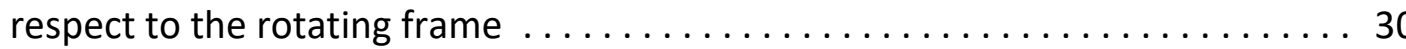

2.6 An example of a GRE sequence. This figure shows the pair of gradients of opposite polarity required to generate the desired phase evolution . . . . . 32

2.7 Example of the FLASH sequence. The GRE is generated by the first two gradients in the frequency encoding direction (see also Fig. 2.4). The rest of the gradient pulses in this sequence (other than the spoilers) are used to encode spatial information into the signal. The squares with the horizontal lines indicate gradient pulses where the amplitude is different each time the

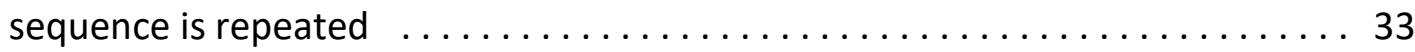

2.8 Example of a spin echo inversion recovery sequence $\ldots \ldots \ldots \ldots \ldots \ldots$

2.9 An example of an EPI-IR sequence diagram $\ldots \ldots \ldots \ldots \ldots \ldots \ldots \ldots \ldots$ 
2.10 Example of a modified Look-Locker inversion recovery sequence. The part of the sequence within the dashed box corresponds to a SSFP sequence known as true Fast Imaging with Steady-state Precision (trueFISP) [35]. The trueFISP readout is repeated for as many $\mathrm{TI}$ values as desired $\ldots \ldots \ldots \ldots \ldots \ldots \ldots$

2.11 Schematic for the calculation of magnetic field outside and inside of an

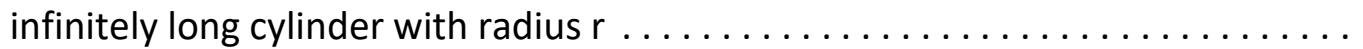

2.12 Example of an acquired AIF, which includes the AIF baseline (left shaded column), the main passage of the GBCA (white box) and the washout (right shaded column). This example was measured in a phantom $\ldots \ldots \ldots \ldots \ldots$

3.1 Representation of the location of the MCA on a pixel grid. (A) The MCA is in the center of four pixels and each pixel contains approximately $60 \%$ MCA, and $40 \%$ other tissues. (B) The MCA is placed in a shifted position, where the right-side pixels contain approximately $71 \%$ of MCA and the two left-side

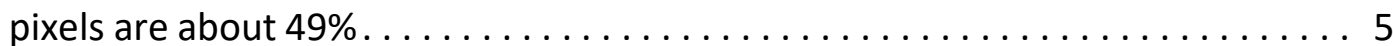

3.2 Circular vessel and ROI model used in phase error calculations: $(\mathbf{A})$ the vessel is centered with respect to the pixel grid; (B) the vessel is shifted by half of a pixel to the right; (C) the vessel is shifted to the right by half of a pixel and

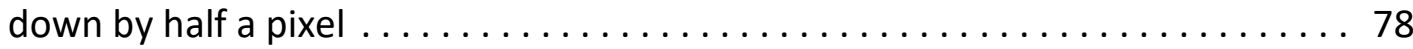

3.3 The square pixel method used in the phase error simulations. Here, the solid square represents the vessel and the dashed plus-sign line represents the

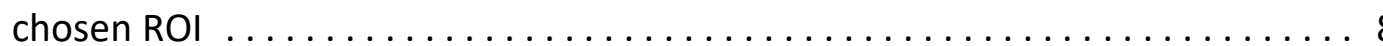

4.1 The difference in acquired MR phase based on a constant experimental error (shown with the vertical arrow). For low signal magnitude data (the solid arrows), the phase uncertainty is much higher than in high signal magnitude

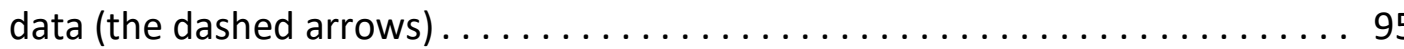

4.2 AlF $_{\mathrm{p}}$ created using Eq. 4.13 and data in Table 4.1. The curve was shifted in time to place the peak AIF value at a time that resembles a clinical acquisition. The temporal resolution was changed from $4.97 \mathrm{~s}$ in the original work to 3.5 $s$ to match the clinical temporal resolution of the DCE-MRI acquisition used in this work .101

4.3 AIF curves derived from SI-calculated (squares) and $\phi$-calculated (diamonds) AIFs computed from the original AIF $_{P}$ curve (line). The three data sets have identical AIF values over the entire imaged range $\ldots \ldots \ldots \ldots \ldots \ldots \ldots \ldots$ 
4.4 (A) The measured phase values from AlF (circles) along with a +0.05 rad (upward triangle) and -0.05 rad (downward triangle) constant error. (B) The calculated $\mathrm{AlF}_{\mathrm{O}}$ values for each of the three curves in $(\mathbf{A})$. Note that the use of Eq. 4.2 removes any baseline error values from the rest of the $A I F_{O}$ curve resulting in uniform AIF values in (B) . . . . . . . . . . . . . . . 107

4.5 (A) The $\phi$ values from AlF $_{\mathrm{P}}$ (circles) along with a linearly-proportional error such that AIF $\phi$-error $=0$ at peak and was +0.10 rad (upward triangle) and $0.10 \mathrm{rad}$ (downward triangle). (B) The calculated AIF values for each of the

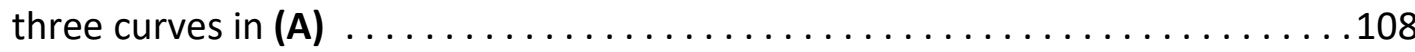

4.6 The $\phi$ values from AIF (circles) along with a linearly-proportional error such that AIF $\phi$-error $=0$ at peak and was +0.50 rad (upward triangle) and -0.50 rad (downward triangle). (B) The calculated AIF values for each of the three curves in (A)

4.7 $\mathrm{AlF}_{\mathrm{O}}$ computation using $\mathrm{SI}$ based on the average of five simulations with defined input SNR values of (A) 3 and (B) 7. Error bars represent the standard deviation of the five simulation results $\ldots \ldots \ldots \ldots \ldots \ldots 11$

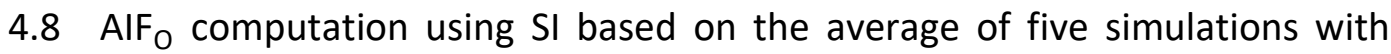
defined input SNR values of (A) 10 and (B) 20. Error bars represent the standard deviation of the five simulation results

4.9 $\mathrm{AlF}_{\mathrm{O}}$ computation using $\mathrm{SI}$ based on the average of five simulations with defined input SNR values of (A) 50 and (B) 100. Error bars represent the standard deviation of the five simulation results

4.10 $\mathrm{AlF}_{\mathrm{O}}$ computation using $\Delta \phi$ based on the average of five simulations with defined input SNR values of (A) 3 and (B) 7. Error bars represent the standard deviation of the five simulation results

4.11 $\mathrm{AlF}_{\mathrm{O}}$ computation using $\Delta \phi$ based on the average of five simulations with defined input SNR values of (A) 10 and (B) 20. Error bars represent the standard deviation of the five simulation results $\ldots \ldots \ldots \ldots \ldots \ldots \ldots \ldots \ldots \ldots$

4.12 $\mathrm{AlF}_{\mathrm{O}}$ computation using $\Delta \phi$ based on the average of five simulations with defined input SNR values of (A) 50 and (B) 100. Error bars represent the standard deviation of the five simulation results

4.13 The effects of PVA on the measurement of the PO AIF in comparison with the PT AIF to the true input, AIF $F_{p}$. The curve was limited to this time-frame (1-6 $\mathrm{min}$ ) to better observe the difference in acquisition values at the AIF peak ..117

5.1 An example of the tube phantoms used to measure $T_{1}$ 
5.2 Schematic of the single-compartment system of the dynamic phantom [82]. AIF data were obtained by placing an ROI within the input tubing as it appeared on the images. The tubing of the input and output were connected to a reservoir of water to simulate the distribution of blood throughout the body. This illustration was used, with permission, by Gabrani-Juma et al. [83] . .127

5.3 A schematic of the dynamic phantom system used during experiments. The blue components indicate modifications from the original phantom and pump system created by Shelley Medical for use in nuclear medicine, MR, and computed tomography. This image was used, with permission, by Gabrani-Juma et al. [83]

5.4 Example of IR SI curves following a single $180^{\circ}$ inversion pulse for both EPI-IR and $\mathrm{MOLLI}_{0}$ for the $0.26 \mathrm{mM}$ static phantom. The solid circles indicate the raw SI values outputted from the scanner, before SI polarity inversion. The

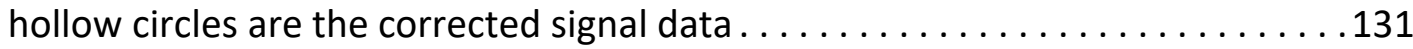

5.5 An example of the inadequate $\mathrm{TI}$ spacing of $\mathrm{MOLLI}_{0}$ to sample the $\mathrm{SI}$ of a high (1.5 $\mathrm{mM})[\mathrm{Gd}]$ phantom and $\mathrm{MOLLI}_{1}$ to sample the $\mathrm{SI}$ of a low $(0.1 \mathrm{mM})[\mathrm{Gd}]$ phantom

5.6 Example of a dynamic phantom AIF. It is characterized by the initial, baseline phase values, a high peak following GBCA injection, a local minimum, a second peak due to recirculation of the GBCA, followed by steady-state

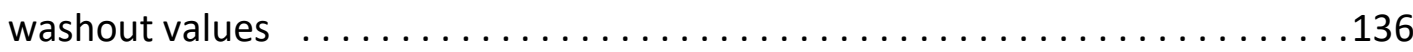

5.7 IR data acquired from static phantoms compared to the literature fit data from Stalder et al. [43] (solid line) . . . . . . . . . . . . . . . . . . . . . 139

5.8 $\mathrm{MOLLI}_{0}$ and $\mathrm{MOLLI}_{1} \mathrm{~T}_{1}^{*}$ data acquired for static phantoms plotted with the $E P I-I R T_{1}$ line of best fit

5.9 $\mathrm{MOLLI}_{0}$ and $\mathrm{MOLLI}_{1} \mathrm{~T}_{1}$ data acquired for static phantoms plotted with the EPI-IR $T_{1}$ line of best fit

5.10 The flow ratio acquired using $\mathrm{MOLLI}_{0}$ and $\mathrm{MOLLI}_{1}$ from the baseline and washout, respectively, for two different measurements. Each column represents the average $T_{1}^{*}$ value over multiple slices and the error bars represent the standard deviation. The horizontal dashed line indicates a constant measurement of $\mathrm{T}_{1}^{*}$ between the two flow states.

5.11 Six different PO AIFs measured using the dynamic phantom system along with the set flow rate. The AIFs were shifted in time so that the peak value occurred at the same time . . . . . . . . . . . . . . . . . . . . . . . . . 143 
5.12 Comparison of [Gd] measurement techniques for concentrations measured in the AIF washout. MOLLI T1 data (using MOLLI $_{\text {comb }}$ ) correlated well with the EPI-IR data.

5.13 The average phantom PO AIF (circles) shown with the newly-formed PT AIF (triangles). The gold-standard AIF washout value determined using EPI-IR (square) was inserted for reference although it was not necessarily measured at the time shown

6.1 Example of an ROI placed on an axial slice over the enhancing tissue volume of an astrocytoma for a $\mathrm{T}_{1}$-weighted anatomical image. Note that the region in between the blue lines of the "figure- 8 " shape (i.e. the enhancing, orange portion) are included in the ROI . . . . . . . . . . . . . . . . . . 161

6.2 The AIF $_{\mathrm{w}}$ for the PO AIF (circles) for each patient as well as AIF $\mathrm{w}_{\mathrm{w}}^{*}$ measured with $\mathrm{T}_{1, \mathrm{w}}^{*}$ (diamonds) and AlF $_{\mathrm{w}}$ measured with $\mathrm{T}_{1, \mathrm{w}}$ (squares). The horizontal dotted, solid, and dashed lines represent the average values of the PO AIF

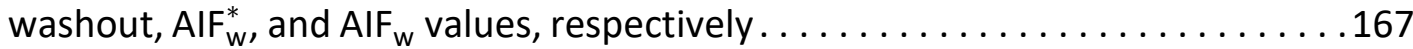

6.3 An example of a PO AIF and PT AIF acquired for patient 54 compared to a literature AIF $\left(\right.$ AIF $\left._{\mathrm{P}}\right)$ [67]. AIF was shifted so that its peak value matched, in time, the peak from the PO and PT AlFs . . . . . . . . . . . . . . . 168

6.4 DCE-MRI parameters for enhancing tumour tissue computed using the two AIF methods for 21 patients with HGG. A significant difference between the two techniques was observed for each DCE-MRI parameter $(* * *$ indicates $P \leq$ 0.001 and $* * P \leq 0.01)$. Error bars represent the standard error of the mean. Parameter units are in $\%$ for $v_{p}, v_{e}$ and $\min ^{-1}$ for $\left.\mathrm{K}^{\text {trans }}, \mathrm{k}_{\mathrm{ep}}\right) \ldots \ldots \ldots \ldots \ldots . \ldots 168$

6.5 Parameter differences between the PO and both PT AIF methods for each patient for $(A) v_{p}$ and $(B) K^{\text {trans }}$

6.6 Parameter differences between the PO and both PT AIF methods for each

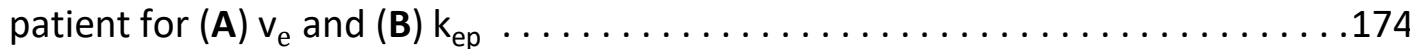




\title{
List of Acronyms and Symbols
}

\author{
AIF Arterial Input Function \\ AIF $_{\mathrm{P}} \quad$ Arterial Input Function from Literature \\ AIF $_{\mathrm{w}} \quad$ Arterial Input Function Washout Value \\ B $_{0} \quad$ External Magnetic Field \\ B $_{1} \quad$ Magnetic Field of Applied RF Pulse \\ BBB Blood Brain Barrier \\ DCE Dynamic Contrast-Enhanced \\ EES Extravascular Extracellular Space \\ EPI Echo Planar Imaging \\ $\mathrm{f}^{\prime} \quad$ Fraction of $\mathrm{k}^{\text {th }}$ Rim-Zone Pixel Inside Vessel \\ $f_{k}^{\prime} \quad$ Ratio of Signal Intensity Between Outside and Inside Pixel k \\ FID Free Induction Decay \\ FLASH Fast Low Angle SHot \\ $f(\theta) \quad$ Cylinder Geometry Factor \\ G Magnetic Field Gradient \\ GBCA Gadolinium-Based Contrast Agent \\ GBM Glioblastoma Multiforme \\ [Gd] GBCA Concentration \\ GRE GRadient Echo \\ h Transport Function \\ Hct Hematocrit Ratio \\ HGG High-Grade Glioma \\ IR Inversion Recovery \\ $\mathrm{k}_{\mathrm{ep}} \quad$ Rate Constant

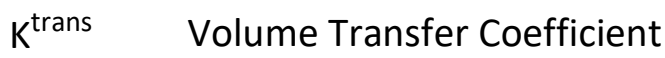 \\ M Magnetization Vector
}




\begin{tabular}{|c|c|}
\hline MOLLI & Modified Look-Locker Inversion Recovery \\
\hline$M_{0}$ & Equilibrium Magnetization \\
\hline $\mathrm{M}_{\mathrm{xy}}$ & Transverse Components of Magnetization \\
\hline $\mathrm{M}_{\mathrm{z}}$ & Longitudinal Component of Magnetization \\
\hline NMR & Nuclear Magnetic Resonance \\
\hline$r_{1}$ & Longitudinal Relaxivity \\
\hline$r_{2}$ & Transverse Relaxivity \\
\hline PO AIF & Phase-Only Arterial Input Function \\
\hline PT AIF & Phase and $T_{1}$ Arterial Input Function \\
\hline PVA & Partial Volume Averaging \\
\hline $\mathrm{RF}$ & Radiofrequency \\
\hline ROI & Region of Interest \\
\hline S & Complex MRI Signal \\
\hline SSFP & Steady-State Free Precession \\
\hline SE & Spin Echo \\
\hline SI & Signal Intensity \\
\hline $\mathrm{S}_{\mathrm{I}}$ & Imaginary Component of MRI Signal \\
\hline SNR & Signal-to-Noise Ratio \\
\hline$S_{R}$ & Real Component of MRI Signal \\
\hline SSS & Superior Sagittal Sinus \\
\hline $\mathrm{T}_{1}$ & Longitudinal Relaxation Time \\
\hline $\mathrm{T}_{1,0}$ & Baseline $T_{1}$ Value \\
\hline $\mathrm{T}_{1}^{*}$ & Apparent Longitudinal Relaxation Time \\
\hline $\mathrm{T}_{2}$ & Transverse Relaxation Time \\
\hline $\mathrm{T}_{2,0}$ & Baseline $T_{2}$ Value \\
\hline $\mathrm{T}_{2}^{*}$ & Effective Longitudinal Relaxation Time \\
\hline TI & Inversion Time \\
\hline $\mathrm{T}_{\mathrm{E}}$ & Echo Time \\
\hline
\end{tabular}




\begin{tabular}{|c|c|}
\hline $\mathrm{T}_{\mathrm{R}}$ & Repetition Time \\
\hline trueFISP & True Fast Imaging with Steady-State Precision \\
\hline$v_{e}$ & EES Volume Fraction of Tissue \\
\hline$v_{p}$ & Blood Plasma Volume Fraction of Tissue \\
\hline VOF & Venous Output Function \\
\hline $\mathrm{Y}$ & Blood Oxygen Concentration \\
\hline$\alpha$ & Flip Angle \\
\hline$\gamma$ & Gyromagnetic Ratio \\
\hline$\theta$ & Angle between $\mathbf{B}_{\mathbf{0}}$ and Vessel \\
\hline$\phi$ & Phase of MR Signal \\
\hline$\phi_{0}$ & Baseline Phase \\
\hline$\phi^{\text {in }}$ & Phase Inside a Vessel \\
\hline$\phi^{\text {out }}$ & Phase Outside a Vessel \\
\hline$\Delta \phi_{\mathrm{F}}$ & Change in Phase Due to Blood Flow \\
\hline$\Delta \phi_{\mathrm{GBCA}}$ & Change in Phase Due to a GBCA \\
\hline$\Delta \phi_{\mathrm{PV}}$ & Phase Error due to Partial Volume Averaging \\
\hline$\phi_{\mathrm{RF}}$ & Phase of RF Pulse \\
\hline$\phi_{\mathrm{w}}$ & Washout Phase \\
\hline$\Delta \phi_{\chi}$ & Change in Phase Due to Magnetic Susceptibility \\
\hline$\rho$ & MR Proportionality Constant \\
\hline$\mu$ & Magnetic Dipole Moment \\
\hline$x$ & Magnetic Susceptibility \\
\hline$x_{\mathrm{e}}$ & Magnetic Susceptibility of Tissue \\
\hline$\chi_{\mathrm{i}}$ & Magnetic Susceptibility Inside Infinite Cylinder \\
\hline$x_{m}$ & Molar Susceptibility of GBCA \\
\hline$\omega_{0}$ & Larmor Frequency \\
\hline$\omega_{\mathrm{RF}}$ & Angular Frequency of RF Pulse \\
\hline $\mathbf{\Omega}$ & Frequency of Rotating Frame \\
\hline
\end{tabular}




\section{Chapter 1}

\section{Introduction}

Cancer is a devastating disease that has affected or will affect most Canadians in their lifetime. According to the Canadian Cancer Society, nearly one out of every two people in Canada is expected to be diagnosed with cancer at some point in their life [1]. From 1988 to 2017 , cancer mortality rates have decreased significantly for both Canadian males (a $32 \%$ decrease) and females (a $17 \%$ decrease) [2]. This is due, primarily, to advances in cancer therapy as well as earlier and more effective cancer detection methods. Currently, the primary method to detect cancer is diagnostic imaging. Since the introduction of magnetic resonance imaging (MRI) as a clinical imaging technology, it has played an important role in the diagnosis and characterization of almost all forms of cancer. MRI has advantages over other imaging methods as it has superior soft tissue contrast relative to $x$-ray imaging methods while not exposing the patient to ionizing radiation. MRI can also produce various types of contrast between tissues based on their specific and unique 
properties. This often means that MRI is the preferred imaging modality when cancer is suspected, for example, for brain tumours such as gliomas.

As tumours develop, they require access to nutrients (e.g. $\mathrm{O}_{2}$ ) and an ability to discard cellular waste products (e.g. $\mathrm{CO}_{2}$ ). These functions are provided by the tumour blood vessels in a process known as perfusion. As tumours grow larger, they require that the vascular network expands along with them. This is done via a process called angiogenesis. However, in most solid tumours, the development of new blood vessels is very erratic and the properties of the blood flow through these vessels as a result is distinct when compared with healthy tissues.

Tumour perfusion can be measured with MRI in several different ways, each one having characteristic advantages and disadvantages. These will be reviewed in Chapter 2 . The work presented in this thesis will focus on one of these perfusion methods known as Dynamic Contrast-Enhanced (DCE)-MRI. DCE-MRI has been shown previously to be a useful imaging method in the diagnosis of various tumours in vivo.

A DCE-MRI procedure involves the injection of a gadolinium-based contrast agent (GBCA) as a tracer to enhance specific areas of interest in the body. With DCE-MRI, valuable information about a tissue is gained by tracking the concentration of the GBCA ([Gd]) as it passes through the tissue. Additionally, the blood flow in an artery that feeds the tissue of interest is measured in what is known as the arterial input function (AIF). With knowledge of the tissue perfusion and the AIF, pharmacokinetic tracer kinetic modeling can be used to get quantitative parameters to characterize the tumour vasculature and blood flow. Accurate knowledge of these properties can help with the 
proper diagnosis of tumours. The passage of the GBCA through a tissue can be tracked via changes to either the magnitude or the phase of the complex MRI signal. At most sites, the use of the magnitude is the preferred approach since most MRI radiologists and technologists have very little experience dealing with the phase of the MR signal. This is unfortunate since using the phase provides more reliable results for a variety of reasons. These two approaches in DCE-MRI will be compared in more detail using simulations in Chapter 4.

However, one drawback of the currently accepted method for using the phase for DCE-MRI is that the accuracy of the results is affected by systematic experimental errors of the phase at baseline (i.e. when there is no GBCA). In Chapter 3, an analysis of the phase error will be presented. Based on the results of this analysis a new, more accurate, method for using the phase of the MRI signal to perform DCE-MRI is proposed in Chapter 4. The novel method was first tested experimentally on plastic phantoms and then with human subjects with high-grade gliomas. The results of these tests are presented in Chapters 5 and 6 , respectively.

The focus of this thesis is to: (i) characterize the errors present when using phase to acquire the AIF, (ii) introduce a novel technique for AIF measurements using a combination of phase and the $T_{1}$ relaxation, and (iii) compare the difference between quantitative DCE-MRI parameters computed using an AIF measured with phase only and an AIF determined using both the phase and $T_{1}$. 


\subsection{Principal Contributions}

In this thesis, several novel contributions to the field of DCE-MRI are presented. First, the derivation of the effect of partial volume averaging (PVA) on the measured phase was theoretically derived and validated with simulations. A novel method to overcome the PVA effects on the AIF was first theoretically established and subsequently validated with both AIF simulations and with test objects. Finally, both the conventional phase-based AIF and the AIF measured with the new approach were used in vivo to observe the effect on the derived DCE-MRI parameters. These promising preliminary clinical results are also consistent with the expectations based on the theoretical predictions. Some of the results given in the thesis were previously presented at conferences [3-5]. 


\section{Chapter 2}

\section{Anatomy and MRI Background}

\subsection{Biology of Gliomas}

Gliomas represent $60 \%$ of all primary brain tumours [6]. Gliomas are very aggressive with no known cure, and only limited progress in disease control has been made in the last several decades [7]. Gliomas consist of astrocytomas, oligodendrogliomas, and a mix of the two (oligoastrocytomas), based on their histological appearance [8]. The World Health Organization classifies the severity of gliomas with a grading system, where grade I and II tumours are non-malignant and grades III and IV are malignant [9]. Malignant gliomas are often referred to as high-grade gliomas (HGG). Grade IV tumours are also known as glioblastoma multiforme (GBM). GBM are the most common form and have the poorest survival rate with $<5 \%$ of patients surviving five years following their diagnosis $[10]$. 


\subsubsection{Glioma Formation}

Gliomas are a direct result of transformed glial cells. Normal brain cell development involves the differentiation of stem cells into progenitor cells and finally into fully differentiated cells. Tumour cells can be derived from multipotent stem cells or committed progenitor cells which then transform into the tumour cells (e.g. astrocytoma, oligodendroglioma) directly [11]. Alternatively, the progenitor cell can form a neuroglial tumour stem cell which then can produce glial tumour cells. Once the glioma cells form, the tumour cells rapidly reproduce and eventually grow enough to develop a structure, complete with vasculature, within the brain.

As mentioned previously, the grade of a glioma dictates the severity of the lesion(s). Grades I and II tend to be composed of well-differentiated cells, with increased cell density and cellular anomalies, or atypias [4]. Higher grade tumours (grades III and IV) are anaplastic, have increased vessel density, atypias, high mitotic activity and a high cell density. GBMs (grade IV tumours) display vascular endothelial proliferation, necrosis, very high cell density, and atypias [4].

One of the key characteristics of tumour formation is the rapid increase in blood vessel creation (capillaries), known as angiogenesis. This allows tumours to get nutrient supply at a higher rate and outgrow the surrounding, healthy tissues. Glioma capillaries specifically show endothelial cell proliferation which is a feature of high-grade gliomas (HGG) $[12,13]$. Glioma cells additionally secrete factors that promote angiogenesis [14]. It has also been shown that glioma cells use this newly added vasculature for malignant purposes and can migrate to different parts of the brain $[15,16]$. The higher blood flow, 
or perfusion, in gliomas is an important factor that allows for their visualization with diagnostic imaging methods, such as magnetic resonance imaging (MRI).

Angiogenesis is one of the hallmarks of tumour development. One of the key features of tumour angiogenesis is the fact that most of the vasculature is produced very quickly, resulting in it being poorly-formed and highly-permeable [17]. For gliomas specifically, this typically results in the blood brain barrier (BBB) - the protective lining of the capillary walls in the brain - being disrupted. One type of molecule that would be able to exit the bloodstream in this instance is an exogenous gadolinium-based contrast agent (GBCA) such as those typically used in MRI. As explained in Section 2.5, the GBCA affects the local MR signal and allows for better observation of blood flow through a tumour. This can be used to obtain information about the vascular permeability and anatomy of the tumour. By tracking a bolus injection of GBCA travelling through tumour vasculature, several quantitative properties of that tumour can be obtained. These properties allow for more effective diagnosis and better characterization of tumours in vivo. Computed tomography (CT) and positron emission tomography (PET) can also be used to obtain similar information about tumour perfusion, using their own respective contrast agents and techniques.

In general, the process of angiogenesis creates a series of blood vessels within an organ, ranging from large (arteries and veins) to small vessels (capillaries). It is the capillary 'bed' that is the most important structure created during angiogenesis as it is the site of cellular respiration. It is where the arteries and veins intersect and a transfer of oxygen and other nutrients from the blood to tissues occurs. Additionally, the capillaries 
extract tissue waste materials, such as carbon dioxide, and excrete them into the venous system.

\subsection{Perfusion}

\subsubsection{Tumour Vasculature Properties}

It is useful to define certain properties of the capillary structure and how they relate to perfusion modelling for MRI. The first is what is known as the permeability-surface area product per unit volume of tissue (PS $)$ for tumour capillaries. $\mathrm{PS}_{\mathrm{a}}$ is actually a product of two terms, where $\mathrm{P}$ is the permeability of the capillaries (in units of $\mathrm{cm} \mathrm{s}^{-1}$ ) and $\mathrm{S}_{\mathrm{a}}$ is the capillary surface area per unit mass of tissue (in units of $\mathrm{cm}^{2} \mathrm{~g}^{-1}$ ). Additionally, it is pertinent to be able to quantify the blood plasma volume per unit volume of tissue, $v_{p}$ (expressed as a percentage of tissue volume). Similarly, the region outside of the vasculature and the cells (i.e. the extravascular extracellular space, EES) can be quantified by $v_{e}$, which is a measure of the EES volume per unit volume of tissue (expressed as a percentage of tissue volume). Finally, the volume transfer coefficient, $\mathrm{K}^{\text {trans }}$, is related to capillary permeability, but has multiple interpretations depending on the significance of the vasculature permeability and the type of perfusion model used. The two most common interpretations of $\mathrm{K}^{\text {trans }}$ are described below.

The Kety model $[18,19]$ assumes that the tissue has a flow-limited uptake of GBCA from the vasculature and that the vascular permeability is high. The original model was developed for inhalation of a gas, and it has been adapted into a blood perfusion model. 
In this model, it is assumed that arterial and venous blood have well-defined, unique GBCA concentrations ([Gd]) and the venous blood has a [Gd] that is in equilibrium with the tissue at all times due to the tissue's high vascular permeability. Therefore, after GBCA injection has occurred, most of the GBCA enter the tissue space. The [Gd] of the intravascular space will then be insignificant compared to the tissue [Gd] and is not taken into account (i.e. $v_{p}=0$ ). In this scenario, $K^{\text {trans }}$ is defined as [20]:

$$
\mathrm{K}^{\mathrm{trans}}=\mathrm{F}_{\mathrm{t}} \rho_{\mathrm{t}}(1-\mathrm{Hct})
$$

where $F_{t}$ is the blood flow rate per unit mass of the respective tissue (in $\mathrm{mL} \mathrm{s}^{-1} \mathrm{~g}^{-1}$ ), $\rho_{t}$ is the density of the tissue (in $\mathrm{g} \mathrm{mL}^{-1}$ ), and Hct is the hematocrit (i.e. the ratio of the volume of red blood cells to the total blood volume). Hct typically ranges between $0.40-0.54$ in adult males and 0.36-0.48 in adult females [21].

The extended Tofts-Kety perfusion model is relevant when vasculature permeability is low; this is equivalently described as the permeability being limited to the $\mathrm{PS}_{\mathrm{a}}$ of the capillaries [22]. This occurs when the blood flow rate is high so that blood plasma can be considered a single pool with equal concentrations in the arteries and veins. The leakage of the GBCA is slow enough to not significantly reduce the intravascular [Gd]. The rate of [Gd] uptake is then determined by the $\mathrm{PS}_{a}$ of the capillary and the difference in blood plasma and EES concentrations. With this model $\mathrm{K}^{\text {trans }}$ is defined as [20]:

$$
\mathrm{K}^{\text {trans }}=\mathrm{PS}_{\mathrm{a}} \cdot \rho_{\mathrm{t}}
$$

Regardless of the perfusion model used, the conventional units of $\mathrm{K}^{\text {trans }}$ are $\mathrm{min}^{-1}$. 


\subsubsection{Perfusion Measurement}

Quantitative MR perfusion methods, such as dynamic contrast-enhanced (DCE)-MRI, use models to track how the GBCA travels into, through, and out of the tumour vasculature and have been shown to be useful methods to diagnose tumours $[23,24]$. For clinical acquisitions, the GBCA is injected into the vascular system at a rate that is not instantaneous. The individual particles of the GBCA travel through the artery at a rate based on the blood flow and vascular structure [25]. The particles' transit is often described by a probability density function known as the transport function, $h(t)$. The [Gd] measured as a function of time, $t$, traveling through an artery feeding the tissue of interest is known as the arterial input function (AIF). The [Gd] in a vein fed by the tissue of interest is the venous output function (VOF). These functions are related by [26]

$$
\operatorname{VOF}(\mathrm{t})=\operatorname{AIF}(\mathrm{t}) * \mathrm{~h}(\mathrm{t})
$$

where $*$ denotes convolution. While the majority of the injected GBCA will pass through the tissue, a portion of it will remain. This quantity is expressed by the residue function, $R(t):$

$$
R(t)=1-\int_{0}^{t} h(\tau) d \tau
$$

Equation 2.4 indicates that the residue function decreases in time based on the definition of $h(t)$. The $[G d]$ can be measured as a function of time in the capillaries $\left([G d]_{c}\right)$, the parenchyma $\left([G d]_{p}\right)$, and for the whole tumour $\left([G d]_{t}\right)$ as the convolution of the AIF and the residue function for each of these volumes $\left(R_{c}, R_{p}\right.$, and $R_{c}+R_{p}$, respectively) multiplied by $F_{t}[26]$ : 


$$
\begin{aligned}
& {[\mathrm{Gd}]_{c}(\mathrm{t})=\mathrm{F}_{\mathrm{t}} \mathrm{R}_{\mathrm{c}}(\mathrm{t}) * \operatorname{AIF}(\mathrm{t}),} \\
& {[\mathrm{Gd}]_{\mathrm{p}}(\mathrm{t})=\mathrm{F}_{\mathrm{t}} \mathrm{R}_{\mathrm{p}}(\mathrm{t}) * \operatorname{AIF}(\mathrm{t}),}
\end{aligned}
$$

and

$$
[G d]_{t}(t)=F_{t}\left(R_{c}(t)+R_{p}(t)\right) * A I F(t) .
$$

Equations 2.5-2.7 assume that the blood and GBCA exist in a 'single compartment' where GBCA molecules from the vascular space are transported into the EES as shown in Fig. 2.1A.

For healthy tissue, where the BBB is intact and no GBCA leakage into the surrounding parenchyma occurs, the residue functions are defined as:

$$
\mathrm{R}_{\mathrm{c}}(\mathrm{t})=0
$$

and

$$
R_{p}(t)=\frac{K^{\text {trans }}}{F_{t}} e^{-k_{e p} t}
$$

$[\mathrm{Gd}]_{\mathrm{t}}(\mathrm{t})$ can be determined, based on Eqs. 2.7-2.9, to be:

$$
[G d]_{t}(t)=K^{\operatorname{trans}} \int_{0}^{t} \operatorname{AIF}(\tau) e^{-k_{e p}(t-\tau)} d \tau
$$

where $k_{e p}$, the rate constant for flow from the EES to the blood plasma, is defined as

$$
\mathrm{k}_{\mathrm{ep}} \equiv \mathrm{K}^{\text {trans }} / \mathrm{v}_{\mathrm{e}}
$$

In gliomas, where the BBB is often compromised, a single compartment model may not be an accurate description of the blood flow. Additionally, the intravascular concentration, as measured by $v_{p}$, is not negligible and must be considered (see Fig. 2.1B). This requires the use of a non-zero vasculature residue function, given by $[22,26]$ : 

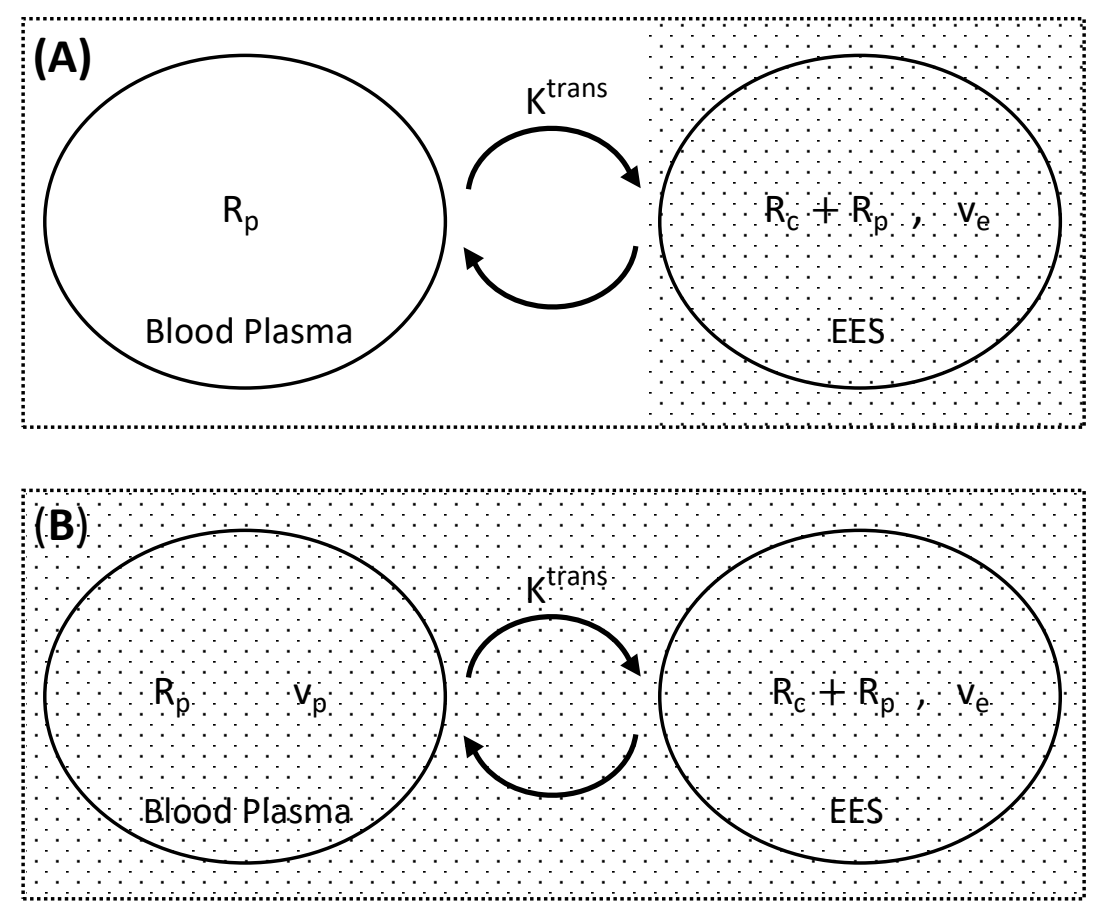

Figure 2.1: The comparison of two perfusion models. (A) The Kety model of perfusion includes a single-compartment where the GBCA moves from the vascular space into the EES. (B) For the extended Tofts-Kety model, the intravascular [Gd] is non-negligible and $v_{p}$ is included. In each image, the dotted background indicates what is included for each voxel. 


$$
R_{c}(t)=\left(\frac{v_{p}}{F_{t}}\right) \delta(t)
$$

where $\delta(t)$ is the Dirac delta function. Using this, the perfusion equation related to this scenario is computed from Eqs. 2.7, 2.9, and 2.12 to be:

$$
[G d]_{t}(t)=v_{p} A I F(t)+K^{\text {trans }} \int_{0}^{t} \operatorname{AIF}(\tau) e^{-k_{e p}(t-\tau)} d \tau
$$

This approach is often referred to as the extended Tofts-Kety model of perfusion [22]. Regardless of which perfusion model is chosen for the DCE-MRI analysis, the acquisition of data must be performed using high spatial and temporal resolutions [26]. The extended Tofts-Kety model is the perfusion-based blood flow model used for this thesis to account for the vascular permeability in HGGs.

\subsection{Basic MRI Theory}

\subsubsection{Magnetic Moments}

MRI is based on the principle of nuclear magnetic resonance (NMR). The NMR signal is generated from the interaction of an atomic nucleus with an external magnetic field. While all nuclei that possess either an odd number of protons or an odd number of protons and neutrons combined can produce an NMR signal, the most abundant and sensitive nucleus from which to clinically acquire MR data is the hydrogen nucleus. In general, nuclei possess an orbital angular momentum, L, and in the classical model of the atom, the nuclei spin about their own axis giving them a spin angular momentum, S. With respect to the total angular momentum $(\mathbf{J}), \mathbf{L}$ is significantly smaller than $\mathbf{S}$, so $\mathbf{J} \approx \mathbf{S}$. The 
"spinning" nuclei generate their own magnetic field, which is represented by the magnetic dipole moment, $\boldsymbol{\mu}$. The relationship between the net magnetization, $\mathbf{M}$, and the magnetic moments for a bulk object is

$$
\mathbf{M}=\sum_{i=1}^{N} \mu_{i}
$$

for a total of $\mathrm{N}$ spins. When an object is placed in an external magnetic field, $\mathbf{B}_{\mathbf{0}}$, the $\boldsymbol{\mu}_{\mathbf{i}}$ experience a torque perpendicular to $\mathbf{B}_{\mathbf{0}}$ which causes them to precess about $\mathbf{B}_{\mathbf{0}}$ in the shape of a cone at a fixed angle of 0.955 rad [27] with its axis parallel to $\mathbf{B}_{\mathbf{0}}$. The precession occurs in a clockwise direction with an angular frequency, $\boldsymbol{\omega}_{0}$, known as the Larmor frequency and given by

$$
\omega_{0}=-\gamma B_{0}
$$

where $\gamma$ is the gyromagnetic ratio and is unique to each element. For hydrogen nuclei, $\gamma$ is $2.675 \times 10^{8} \mathrm{rad} \mathrm{s}^{-1} \mathrm{~T}^{-1}$ [28].

In the absence of an external magnetic field, the $\boldsymbol{\mu}_{\mathbf{i}}$ are randomly oriented due to their thermal energy so no net magnetization exists. In the presence of a magnetic field, $\mathbf{B}_{\mathbf{0}}=\mathbf{B}_{0} \hat{\mathbf{z}}$, this randomness is disrupted and the spins are arranged into two energy states. The spins in the lower energy state align such that the $\mu_{\mathrm{zi}}$ are parallel to $\mathbf{B}_{\mathbf{0}}$ while the higher energy state spins align anti-parallel to $\mathbf{B}_{\mathbf{0}}$. The number of spins in each state is dictated by the Boltzmann distribution [29]:

$$
\frac{\mathrm{N}_{+}}{\mathrm{N}_{-}}=\exp \left[-\frac{\Delta \mathrm{E}}{\mathrm{k}_{\mathrm{B}} \mathrm{T}}\right]
$$

where $\mathrm{N}_{+}$is the number of spins parallel to $\mathbf{B}_{\mathbf{0}}, \mathrm{N}_{-}$is the number of spins anti-parallel to $\mathbf{B}_{0}, \Delta E$ is the energy difference between the two states $\left(\Delta E=\gamma \hbar B_{0}\right), k_{B}$ is the Boltzmann 
constant, and $\mathrm{T}$ is the absolute temperature of the system. Since a larger number of spins will be in the lower energy state and there is no preferred orientation in the plane perpendicular to $\mathbf{B}_{\mathbf{0}}, \mathbf{M}$ will also be parallel to $\mathbf{B}_{\mathbf{0}}$.

\subsubsection{MRI Signal Induction}

MR signal is obtained by measuring the production of current in a series of metal loops, or coils, due to a changing magnetic field as dictated by Faraday's Law [30]. Conventionally in $\mathrm{MRI}$, the direction of $\mathbf{B}_{\mathbf{0}}$ is taken to be the z-direction and the signal detection coils are configured to be sensitive to magnetic field changes in the $x-y$ plane. At equilibrium, even though the individual $\boldsymbol{\mu}_{\mathbf{i}}$ precess about $\mathbf{B}_{\mathbf{0}}$, the net magnetization is static and no current is induced in the detection coils.

If radiofrequency (RF) radiation is applied to the spin system with angular frequency $\omega_{\mathrm{RF}} \approx \omega_{0}$, where $\omega_{0}$ is the Larmor frequency of the spin system (Eq. 2.15), the orientation of $\mathbf{M}$ can be changed from the initial longitudinal direction (i.e. parallel to $\mathbf{B}_{\mathbf{0}}$ ). When $\omega_{\mathrm{RF}} \approx \omega_{0}$ the system is said to be on-resonance. The application of RF energy will cause a coherent transition of spins from the lower energy state to the higher energy state as well as create a phase coherence amongst the spins. The number of spins that will change states is based on the strength and duration of the applied radiation. RF radiation is only applied for short durations, so the term 'RF pulse' is typically used to describe the event. After the RF pulse, the components of the $\boldsymbol{\mu}_{\mathbf{i}}$ in the transverse plane (i.e. perpendicular to $\mathbf{B}_{\mathbf{0}}$ ) will no longer cancel meaning that $\mathbf{M}$ will have a component in the transverse plane that will precess about $\mathbf{B}_{\mathbf{0}}$. This time dependent magnetic field is 
what induces the current in the detection coils.

In a reference frame external to the spin system, referred to as the laboratory reference frame', the precession of $\mathbf{M}$ in a magnetic field $\mathbf{B}$ is dictated by [27]

$$
\frac{\mathrm{d} \mathbf{M}}{\mathrm{dt}}=\gamma \mathbf{M} \times \mathbf{B}
$$

In a reference frame that rotates at the Larmor frequency, $\mathbf{M}$ will rotate at the same frequency as the reference frame when $\mathbf{B}=\mathbf{B}_{\mathbf{0}}$ and will thus be perceived as static. This 'rotating reference frame' can be very convenient for describing the system. Note that in this thesis the axes in the laboratory reference frame will be written as $x, y$, and $z$ whereas for the rotating frame the notation $x^{\prime}, y^{\prime}$, and $z^{\prime}$ will be used.

The equation of motion for $\mathbf{M}$ in the rotating frame is given by [30]

$$
\frac{\mathrm{d} \mathbf{M}}{\mathrm{dt}}=\gamma \mathbf{M} \times\left(\mathbf{B}+\frac{\Omega}{\gamma}\right)
$$

where $\Omega / \gamma$ is known as a 'fictitious field' that translates the motion of $\mathbf{M}$ from the laboratory frame to the rotating frame and $\mathbf{B}$ is the magnetic field experienced by the magnetic moments. It is often the case that $\mathbf{B}=\mathbf{B}_{\mathbf{0}}$, but other important contributions sometimes need to be considered. When $\boldsymbol{\Omega}$ is set equal to $\boldsymbol{\omega}_{\mathbf{0}}=-\omega_{0} \hat{\mathbf{z}}$ and $\mathbf{B}=\mathbf{B}_{\mathbf{0}}$, it can be seen from Eq. 2.18 that $\mathbf{M}$ is static in the rotating frame. This is true independently of the orientation of $\mathbf{M}$.

In MRI it is advantageous to use circularly polarized RF pulses. Such a pulse in the laboratory frame can be written as [27]

$$
B_{1}(t)=B_{1}(t)\left[\cos \left(\omega_{R F} t+\phi_{R F}\right) \hat{x}-\sin \left(\omega_{R F} t+\phi_{R F}\right) \hat{y}\right]
$$

where $\phi_{\mathrm{RF}}$ is the phase of the RF pulse and $B_{1}(t)$ is a time-dependent modulation of the 
pulse which, in the simplest case, corresponds to turning the pulse on and off. This can also be written in complex notation as

$$
B_{1}^{c}(t)=B_{1}(t) e^{-i\left(\omega_{R F} t+\phi_{R F}\right)}
$$

where the real axis corresponds to the x-axis in the laboratory frame. In the on-resonance rotating frame with $\phi_{\mathrm{RF}}=0$, the equations for the RF pulse simplify to

$$
B_{1}(t)=B_{1}(t) \hat{\mathbf{x}}^{\prime}
$$

and

$$
\mathrm{B}_{1}^{\mathrm{c}}(\mathrm{t})=\mathrm{B}_{1}(\mathrm{t})
$$

The equation of motion for $\mathbf{M}$ in the rotating frame during the application of an RF pulse, for $B=B_{0}+B_{1}(t)$, is given by

$$
\frac{\mathrm{d} \mathbf{M}}{\mathrm{dt}}=\gamma \mathbf{M} \times\left(\mathbf{B}_{0}+\mathbf{B}_{1}(\mathrm{t})+\frac{\boldsymbol{\Omega}}{\gamma}\right) .
$$

As noted above, the effect of the RF pulse is to tip the magnetization away from the zaxis. When the angular frequency of the rotating frame, $\boldsymbol{\Omega}$, is set equal to $-\gamma \mathbf{B}_{0}$, the effective magnetic field in the rotating frame equals $\mathbf{B}_{\mathbf{1}}(\mathrm{t})$. By analogy with the laboratory frame situation, it can be seen that the spins will precess about the $x^{\prime}$-axis, away from their orientation parallel to $\hat{\mathbf{z}}$ towards the $\mathbf{x}^{\prime}-\mathrm{y}^{\prime}$ plane. The subsequent angle between $\mathbf{M}$ and $\mathbf{B}_{\mathbf{0}}$ after $\mathbf{B}_{\mathbf{1}}$ is applied is known as the flip angle, $\boldsymbol{\alpha}$, and is given by [27]:

$$
\alpha=\int_{0}^{T_{R F}} \gamma B_{1}(t) d t
$$

where $T_{R F}$ is the duration of the RF pulse. Such an RF pulse is often referred to as an $\alpha$ pulse. An RF pulse with $\alpha=\pi / 2$ rad provides the greatest amount of MR signal, (assuming complete $T_{1}$ relaxation is allowed), as the largest component of $\mathbf{M}$ is moved into the $\mathbf{x}^{\prime}-\mathrm{y}^{\prime}$ 
plane. This component of $\mathbf{M}$ is the transverse magnetization $\left(\mathbf{M}_{\mathbf{x y}}\right)$. When $\mathbf{B}_{\mathbf{1}}(\mathrm{t})=\mathrm{B}_{\mathbf{1}}(\mathrm{t}) \hat{\mathbf{x}}^{\prime}$, a $\pi / 2$ pulse tips the magnetization which is initially parallel to $\hat{\mathbf{z}}^{\prime}$, to an orientation which is parallel to $\hat{\mathbf{y}}^{\prime}$. When $\alpha=\pi \mathrm{rad}$, the magnetization becomes inverted (i.e. $M \hat{z}$ to $-M \hat{z}$ ). Such a pulse is called an inversion pulse.

In the rotating reference frame, after $\mathbf{M}$ has been rotated away from $\mathbf{B}_{\mathbf{0}}$ and the RF pulse is no longer acting on it, $\mathbf{M}$ will relax back to its equilibrium state parallel to $\mathbf{B}_{\mathbf{0}}$. This is a result of spins that were previously excited to the higher energy state returning down to their base state. $\mathbf{M}$ will return from its position following an RF pulse to the longitudinal axis at a rate dictated by the $T_{1}$ time constant, or the longitudinal relaxation time, of the sample. Additionally, $\mathbf{M}_{\mathbf{x y}}$ will immediately begin to decay from its maximum value as the spins dephase throughout the transverse plane. This rate of transverse magnetization decay is dictated by the $T_{2}$ time constant. The equation of motion of $\mathbf{M}$ undergoing $T_{1}$ and $T_{2}$ relaxations in the laboratory frame is given by the phenomenological Bloch equation [27,30]:

$$
\frac{\mathrm{d} \mathbf{M}}{\mathrm{dt}}=\gamma \mathbf{M} \times \mathbf{B}-\frac{\mathrm{M}_{\mathrm{x}} \hat{\mathbf{x}}+\mathrm{M}_{\mathrm{y}} \hat{\mathbf{y}}}{\mathrm{T}_{2}}-\frac{\mathrm{M}_{\mathrm{z}}-\mathrm{M}_{0}}{\mathrm{~T}_{1}} \hat{\mathbf{z}},
$$

where $\mathbf{B}=\mathbf{B}_{\mathbf{0}}+\mathbf{B}_{1}, \mathrm{M}_{\mathbf{x}}$ and $\mathrm{M}_{\mathrm{y}}$ are the separated components of $\mathbf{M}_{\mathbf{x y}}$, and $\mathbf{M}_{0}$ is the magnetization at equilibrium. The solution to the differential equation in Eq. 2.25 provides $\mathbf{M}$ in both longitudinal and transverse planes as a function of time, immediately following an RF pulse in the laboratory frame [30]:

$$
M_{z}(t)=M_{0}\left(1-e^{-t / T_{1}}\right)+M_{z}(\varepsilon) e^{-t / T_{1}}
$$

and 


$$
M_{x y}(t)=M_{x y}(\varepsilon) e^{-t / T_{2}} e^{-i \omega_{0} t}
$$

Here, $M_{z}(\varepsilon)$ and $M_{x y}(\varepsilon)$ represent the respective longitudinal and transverse magnetizations at a time $\varepsilon$ immediately following the RF pulse. These equations can be represented in terms of their corresponding equations in the rotating reference frame by:

$$
M_{z^{\prime}}(t)=M_{0}\left(1-e^{-t / T_{1}}\right)+M_{z^{\prime}}(\varepsilon) e^{-t / T_{1}}
$$

and

$$
M_{x^{\prime} y^{\prime}}(t)=M_{x^{\prime} y^{\prime}}(\varepsilon) e^{-t / T_{2}}
$$

As expected, there is no difference in the $M_{z}(t)$ term between the two reference frames.

If the initial $\alpha$-pulse of an MR sequence is applied in the $x^{\prime}$-direction (see Eq. 2.21) then at time $t=\varepsilon$ the transverse components of $\boldsymbol{\mu}_{\mathbf{i}}$ are aligned parallel to $\hat{y}^{\prime}$ and

$$
\mathbf{M}_{\mathrm{x}^{\prime} \mathrm{y}^{\prime}}(\varepsilon)=\mathrm{M}_{0} \sin \alpha \hat{\mathbf{y}}^{\prime}
$$

At $t=\varepsilon$, the spins are said to be in phase. For times $t>\varepsilon$, the spins will no longer be in phase since the $\boldsymbol{\mu}_{\mathbf{i}}$ experience slightly different magnetic fields (and resonance frequencies) due to imperfections in the applied magnetic field and contributions from other magnetic moments in the local environment of each nuclear spin. This dephasing causes an attenuation of the magnetization, which can be written as

$$
M_{x^{\prime} y^{\prime}}(t)=M_{0} \sin \alpha e^{-t / T_{2}^{*}}
$$

$T_{2}^{*}$ is the effective $T_{2}$ time constant given by

$$
\frac{1}{T_{2}^{*}}=\frac{1}{T_{2}}+\gamma \Delta B_{0},
$$

where $\Delta \mathrm{B}_{0}$ is the inhomogeneity of the $\mathrm{B}_{0}$ field. 


\subsubsection{MR Phase}

The magnitude of the magnetization is only one component of the total information obtained when performing an MR acquisition. Immediately following the application of an on-resonance $\pi / 2$ RF pulse, the phase of $M_{x y}$, for the purposes of this discussion, is defined as zero and the spins are considered to be pointing solely in the $y^{\prime}$-direction. In the laboratory frame, the spins will immediately dephase (see Fig. 2.2 ) in the transverse plane and the total phase, $\phi$, can be determined based on the change from the original phase, $\phi_{i}$, by

$$
\phi(t)=\phi_{i}-\int_{0}^{t} \omega \mathrm{dt}^{\prime}=\phi_{\mathrm{i}}-\gamma \mathrm{Bt}
$$

where the negative sign indicates rotation in the clockwise direction. More generally, the phase of a bulk object is not uniform over an entire object due to inhomogeneities in the main magnetic field. This can cause the main field to be different from the applied field and to vary with position and time, denoted as $\Delta B(r, t)$, such that the total phase in the rotating frame is then

$$
\phi(r, t)=-\int_{0}^{t} \gamma \Delta B\left(r, t^{\prime}\right) d t^{\prime}
$$

It is often useful to measure the change in phase, $\Delta \phi$, between two points in time:

$$
\Delta \phi(\mathrm{t})=\phi(\mathrm{t})-\phi_{\mathrm{i}}
$$

\subsubsection{Complex MR Signal}

The existence of both MR signal magnitude and phase lends itself to a complex 

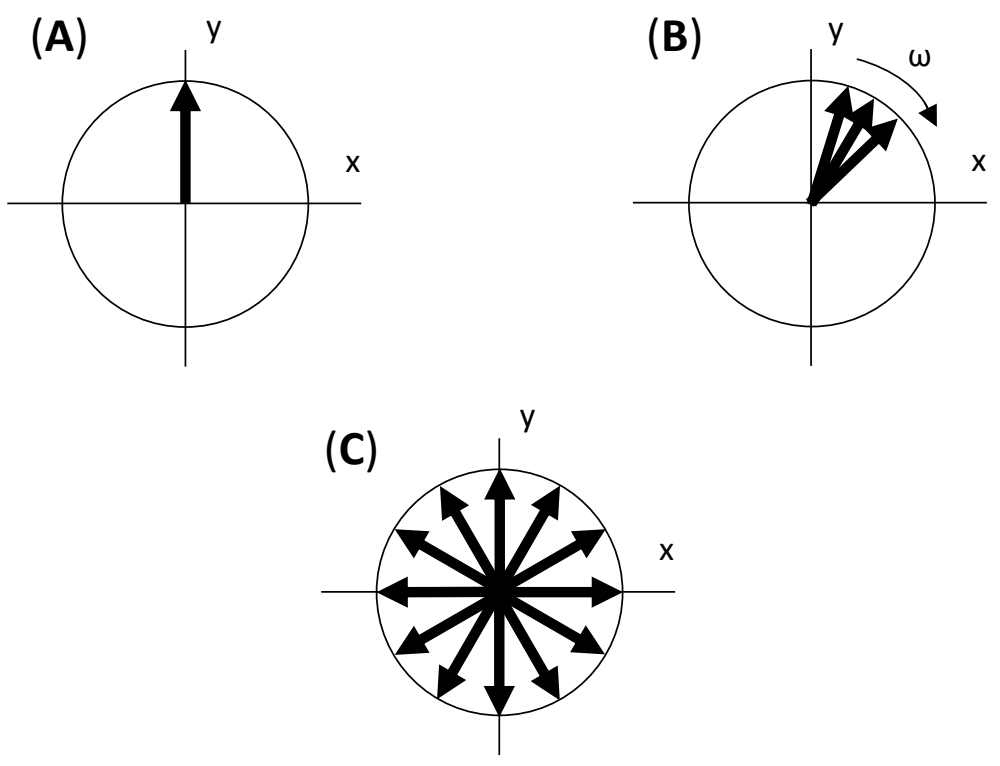

Figure 2.2: Motion of the magnetization after being tipped $(\mathbf{A})$ from the longitudinal into the transverse plane. (B) The spins will dephase at different rates. (C) Eventually, the net transverse magnetization goes to zero.

representation of the signal in the transverse plane. An example of the MR signal representation is provided in Fig. 2.3. The measured signal lies in the complex plane where the $x^{\prime}$ - and $y^{\prime}$-axes are taken to be real and imaginary axes, respectively. If the signal component along the real axis is $S_{R}$ and the component along the imaginary axis is $\mathrm{S}_{1}$, then the magnitude of the complex signal, also known as the signal intensity (SI), is then defined as

$$
S I=|S|=\sqrt{S_{R}^{2}+S_{I}^{2}} .
$$

It is this magnitude of the MR signal that is normally displayed in MR images. From this equation, the measured signal intensity data will be expressed simply as SI, unless otherwise stated. Additionally, the phase can be defined as the angle between the real 


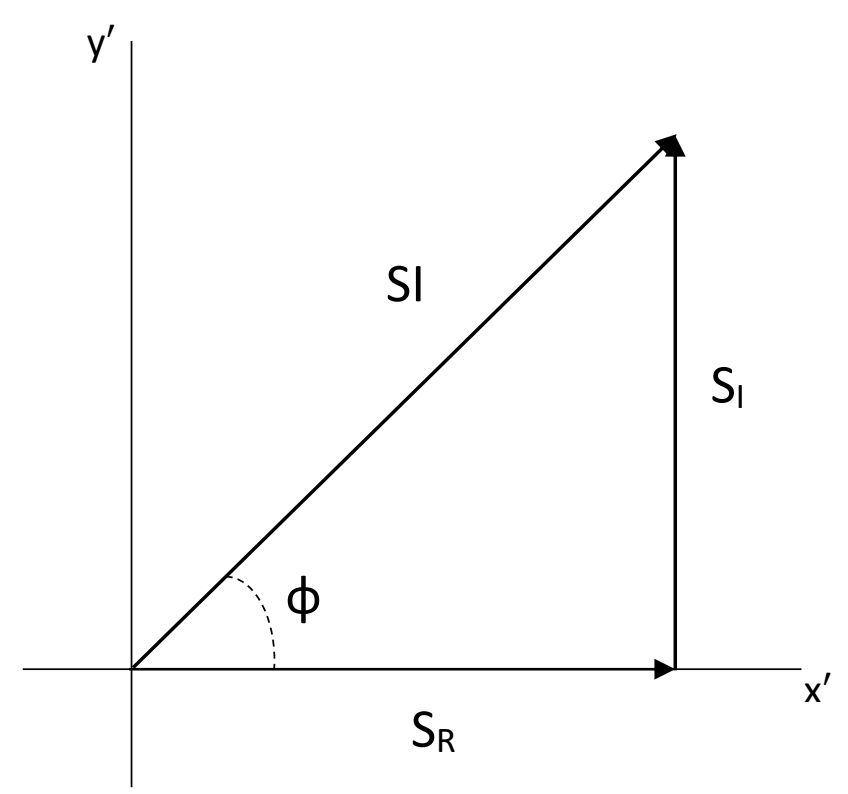

Figure 2.3: Schematic representation of the MR signal in the complex plane.

axis and the signal vector and can be computed from the real and imaginary signal components by:

$$
\phi=\tan ^{-1}\left(\frac{\mathrm{S}_{\mathrm{I}}}{\mathrm{S}_{\mathrm{R}}}\right)
$$

\subsection{MR Sequences}

MR data are acquired using combinations of RF pulses, timing techniques, and magnetic field gradient pulses. These "combinations" are what are known as MR sequences. A sequence diagram for a basic $M R$ sequence is shown in Fig 2.4. If an $N \times N$ image is desired, the basic sequence block must be repeated $\mathrm{N}$ times with one MR signal being acquired each time. This resulting data set is in a spatial frequency space known as kspace and each MR signal is referred to as a line of k-space. The image is then obtained 
by performing a 2D Fourier transform on this data set.

All MRI sequences begin with an $\alpha$-pulse that tips $\mathbf{M}$ such that it is no longer parallel to $\mathbf{B}_{\mathbf{0}}$. This creates a non-zero component of $\mathbf{M}$ in the transverse plane which precesses about $\mathbf{B}_{\mathbf{0}}$ causing a signal to be induced in the detection coil. This signal, known as the free induction decay (FID), is generated by all MRI sequences but it is not normally feasible to use the FID signal to create the image. Echo sequences are normally used instead. An echo is the refocused magnetization in the transverse plane after the initial signal dephasing [30]. Echoes can be obtained using RF pulses (such an echo is referred to as a 'spin echo', SE) or by rapidly changing magnetic field gradients (these echoes are known as 'gradient echoes', GRE). SE and GRE sequences each have certain advantages relative to the other and both are important since they have different dependences on the properties of the tissues.

To generate an MR image from a set of MR signals, spatial information must be encoded into the signals. This is accomplished with the use of gradient pulses such as the ones shown in Fig. 2.4. Since these gradients are imposed for a short period of time (on the order of $\mathrm{ms}$ ) they are referred to as magnetic field gradient pulses, or just gradient pulses. The gradient pulses selectively vary the total magnetic field in the direction of $\mathbf{B}_{\mathbf{0}}$ as a function of position in the $x_{-}^{-}, y^{-}$, or $z$-direction. The contribution to the total magnetic field due to the magnetic field gradient $(\mathbf{G})$, defined as $\mathbf{B}_{\mathbf{G}}$, is given by

$$
\mathbf{B}_{\mathbf{G}}=(\mathbf{G} \cdot \mathbf{r}) \hat{\mathbf{z}}
$$

or

$$
B_{G}=\left(G_{x} x+G_{y} y+G_{z} z\right) \hat{z}
$$




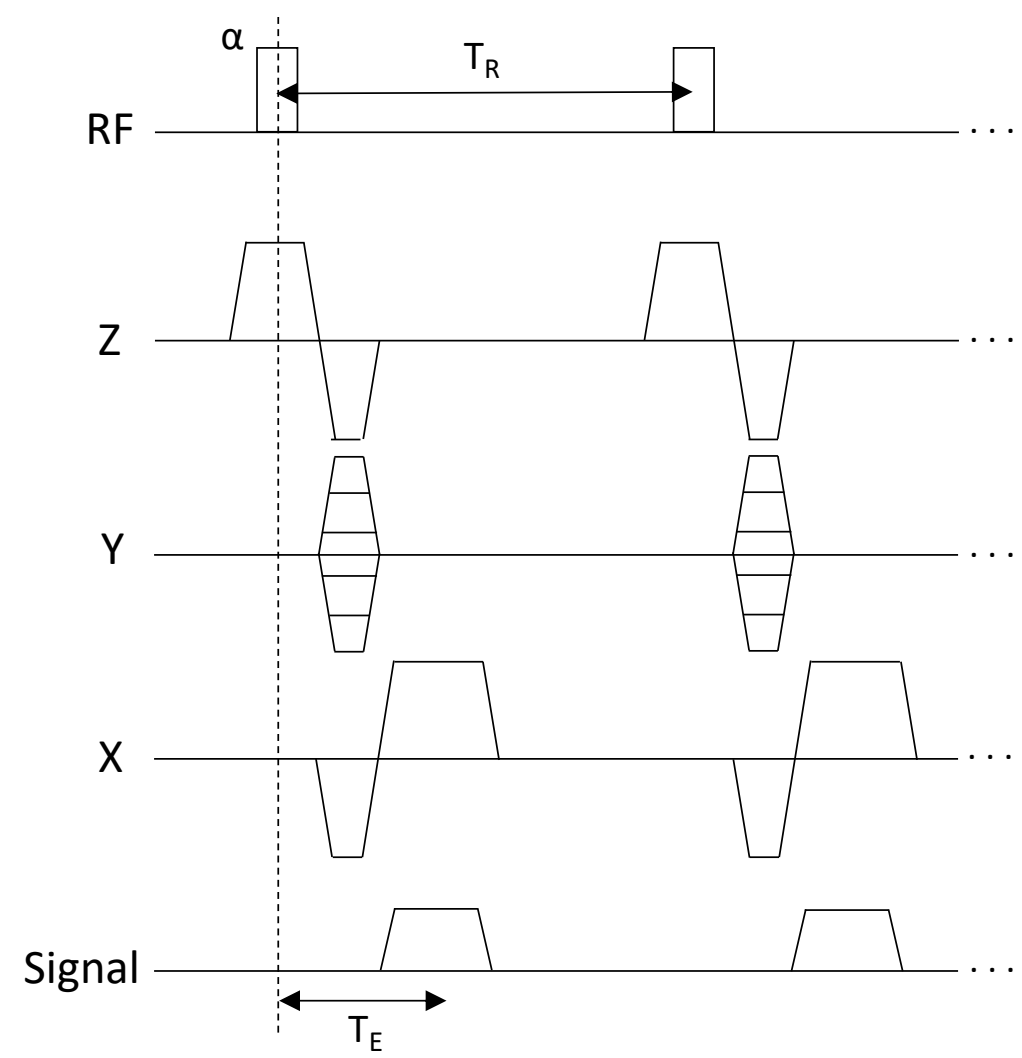

Figure 2.4: A sequence diagram for a basic gradient echo MR sequence. Gradient pulses are shown as trapezoidal objects. The gradient pulses for the $\mathrm{Y}$-axis have different amplitudes each time the basic sequence block is repeated. $T_{R}$ and $T_{E}$ are the repetition time and echo time, respectively. The example shown is for the acquisition of an axial image.

where $G_{x}, G_{y}$, and $G_{z}$ are the $x_{-}, y^{-}$, and z-gradients, respectively. It can be seen from these equations that the units for the gradients are in Tesla per unit distance. When the gradients are applied, the total magnetic field is given by:

$$
\mathbf{B}=\left(B_{0}+\mathbf{G} \cdot \mathbf{r}\right) \hat{\mathbf{z}}
$$

In a traditional 2D MRI sequence, such as the one depicted in Fig. 2.4, the first part of the sequence determines the plane of the image to be generated. This process, normally referred to as slice selection, will be considered in more detail in the following 
section. Encoding the spatial information into the measured signal about where the signal comes from in the transverse plane traditionally involves processes referred to as frequency encoding and phase encoding. For the sequence shown in Fig. 2.4, which would be used to acquire an axial image, slice selection, phase encoding, and frequency encoding are shown on the $Z, Y$, and $X$ axes, respectively.

With a 2D MRI sequence, images can be acquired in the $x-y$ plane (axial), the $x-z$ plane (coronal), or the $y-z$ plane (sagittal). They can also be acquired in a plane which is rotated relative to one of the primary planes (oblique) or even rotated from an oblique plane (double oblique).

\subsubsection{Slice Selection}

For MR sequences, it is normally desirable that the RF pulses excite only part of the object being studied at a time. This is accomplished by applying a gradient pulse at the same time as the RF pulse (see the z-axis in Fig. 2.4). Each RF pulse will have a certain bandwidth of frequencies. When applied in the presence of a magnetic field gradient, the RF pulse will excite the spins in a region of the object where the resonance frequency of the spins falls within the bandwidth of the RF pulse. This is known as the slice selection process.

When $\mathbf{B}_{\mathbf{1}}=\mathrm{B}_{1}(\mathrm{t}) \hat{\mathbf{x}}^{\prime}$ is applied in the presence of a slice selection gradient in the $\mathbf{z}-$ direction, given by

$$
\mathbf{B}_{\mathbf{G}}=(\mathbf{G} \cdot \mathbf{r}) \hat{\mathbf{z}}^{\prime}=\mathrm{G}_{\mathrm{z}} \mathrm{z} \hat{\mathbf{z}}^{\prime}
$$

the equation of motion (Eq. 2.23) can be written as 


$$
\frac{\mathrm{d} M(r, t)}{\mathrm{dt}}=\gamma M(r, t) \times\left[B_{0}+B_{G}+B_{1}(t)+\frac{\Omega}{\nu}\right] .
$$

If the angular frequency of the rotating frame, $\boldsymbol{\Omega}$, is set equal to the angular frequency of the RF pulse that corresponds to the position of the centre of the slice that is being excited, $z_{c}$, then

$$
\mathbf{\Omega}=-\left(\omega_{0}+\gamma G_{z} z_{c}\right) \hat{z}^{\prime}
$$

and Eq. (2.42) can be written as

$$
\begin{aligned}
\frac{d \mathbf{M}(r, t)}{d t} & =\gamma M(r, t) \times\left\{B_{1}(t) \hat{\mathbf{x}}^{\prime}+\left[B_{0}+G_{z} z-\frac{\left(\omega_{0}+\gamma G_{z} z_{c}\right)}{\nu}\right] \hat{\mathbf{z}}^{\prime}\right\} \\
& =\gamma M(r, t) \times\left[B_{1}(t) \hat{\mathbf{x}}^{\prime}+G_{z}\left(z-z_{c}\right)\right] \hat{\mathbf{z}}^{\prime} .
\end{aligned}
$$

This can also be written as:

$$
\begin{aligned}
& \frac{d M_{x^{\prime}}(r, t)}{d t}=\gamma G_{z}\left(z-z_{c}\right) M_{y^{\prime}}(r, t) \\
& \frac{d M_{y^{\prime}}(r, t)}{d t}=-\gamma G_{z}\left(z-z_{c}\right) M_{x^{\prime}}(r, t)+\gamma B_{1}(t) M_{z^{\prime}}(r, t) \\
& \frac{d M_{z^{\prime}}(r, t)}{d t}=-\gamma B_{1}(t) M_{y^{\prime}}(r, t) .
\end{aligned}
$$

This set of equations is very difficult to solve analytically. They can, however, be simplified by introducing the assumption that $\sin \alpha \approx \alpha$. Strictly speaking, this simplification is only valid for $\alpha$ values of a few degrees, but in practice it usually gives useful solutions even for $\pi / 2$ pulses.

In the small $\alpha$ approximation, $M_{z^{\prime}}(r, t)=M_{z}^{0}(r)$, where $M_{z}^{0}(r)$ is the initial equilibrium magnetization. Therefore, the resulting equations of motion become: 


$$
\begin{aligned}
& \frac{d M_{x^{\prime}}(r, t)}{d t}=\gamma G_{z}\left(z-z_{c}\right) M_{y^{\prime}}(r, t) \\
& \frac{d M_{y^{\prime}}(r, t)}{d t}=-\nu G_{z}\left(z-z_{c}\right) M_{x^{\prime}}(r, t)+\gamma B_{1}(t) M_{z}^{0}(r) \\
& \frac{d M_{z^{\prime}}(r, t)}{d t}=0 .
\end{aligned}
$$

The first two of these equations can be combined to get a single equation for the complex function $M_{x^{\prime} y^{\prime}}(r, t)$, defined by

$$
M_{x^{\prime} y^{\prime}}(r, t) \equiv M_{x^{\prime}}(r, t)+i M_{y^{\prime}}(r, t)
$$

to get:

$$
\begin{aligned}
\frac{d M_{x^{\prime} y^{\prime}}(r, t)}{d t} & =-i \gamma G_{z}\left(z-z_{c}\right)\left[M_{x^{\prime}}(r, t)+i M_{y^{\prime}}(r, t)\right]+i \gamma B_{1}(t) M_{z}^{0}(r) \\
& =-i \gamma G_{z}\left(z-z_{c}\right) M_{x^{\prime} y^{\prime}}(r, t)+i \gamma B_{1}(t) M_{z}^{0}(r) .
\end{aligned}
$$

This equation can be solved analytically using the method of undetermined multipliers. For details, the reader is referred to ref. [31].

The solution to this differential equation can be written as:

$$
\begin{array}{r}
\frac{M_{x^{\prime} y^{\prime}}(z, t)}{\gamma M_{z}^{0}(t)}=i \exp \left[-i \gamma \int_{0}^{t} G_{z}\left(t^{\prime}\right)\left(z-z_{c}\right) d t^{\prime}+i \gamma G_{z}\left(z-z_{c}\right) \frac{T_{R F}}{2}\right] \times \\
\int_{-\infty}^{\infty} B_{1}\left(\tau+\frac{T_{R F}}{2}\right) e^{i \gamma G_{z}\left(z-z_{c}\right) \tau} d \tau,
\end{array}
$$

where $T_{R F}$ again is the duration of the RF pulse. The integral can be seen to be an inverse Fourier Transform where the temporal frequency is given by

$$
f_{c} \equiv \forall G_{z}\left(z-z_{c}\right)
$$

and $\forall$ is $\gamma / 2 \pi$. Thus Eq. 2.49 can also be written as 


$$
\begin{gathered}
\frac{M_{x^{\prime} y^{\prime}}(z, t)}{\gamma M_{z}^{0}(t)}=i \exp \left[-i \nu \int_{0}^{t} G_{z}\left(t^{\prime}\right)\left(z-z_{c}\right) d t^{\prime}+i \gamma G_{z}\left(z-z_{c}\right) \frac{T_{R F}}{2}\right] \times \\
F T^{-1}\left\{B_{1}\left(\tau+\frac{T_{R F}}{2}\right)\right\}_{f=f_{c}} .
\end{gathered}
$$

It is normally the case that $B_{1}$ is real and even with respect to the centre of the pulse (i.e. $\tau=0$ in Eq. 2.49). When this is true, the magnitude of $M_{x^{\prime} y^{\prime}}(z, t)$ is given by:

$$
\left|M_{x^{\prime} y^{\prime}}(z, t)\right|=\gamma M_{z}^{0}(z) F T^{-1}\left\{B_{1}\left(\tau+\frac{T_{R F}}{2}\right)\right\}_{f=f_{c}}
$$

and the phase, $\phi(z, t)$, is:

$$
\phi(z, t)=-\nu \int_{0}^{t} G_{z}\left(t^{\prime}\right)\left(z-z_{c}\right) d t^{\prime}+\gamma G_{z}\left(z-z_{c}\right) \frac{T_{R F}}{2}+\frac{\pi}{2}
$$

since $\mathrm{i}=\mathrm{e}^{\mathrm{i} \pi / 2}$. This can also be written as

$$
\phi(z, t)=\gamma\left(z-z_{c}\right)\left[-\int_{0}^{t} G_{z}\left(t^{\prime}\right) d t^{\prime}+G_{z} \frac{T_{R F}}{2}\right]+\frac{\pi}{2}
$$

Equation 2.52 shows that the slice profile is directly proportional to the inverse Fourier Transform of the modulation function for the RF pulse. Since the desired slice profile normally requires uniform excitation across the slice of interest and no excitation elsewhere, this type of excitation corresponds to a rect function. Therefore, to get this slice profile function, the RF pulse needs to be modulated by a sinc function.

As mentioned in Section 2.3.2, the effect of an on-resonance RF pulse applied in the $\hat{x}^{\prime}$-direction is to tip the magnetization from its initial orientation parallel to $\hat{\mathbf{z}}^{\prime}$ towards the $\hat{y}^{\prime}$-axis. In other words, the phase in the on-resonance rotating frame should be $\pi / 2$. It can be seen from Eq. 2.54 that this is the case for $z=z_{c}$, but not true for other locations 
in the slice. To remove this unwanted phase shift for $z \neq z_{c}$, an extra gradient pulse can be applied in the slice selection direction. This gradient pulse should be equal in amplitude and opposite in polarity to the initial slice selection gradient and its duration should be $\frac{1}{2} T_{R F}$. From Eq. 2.54, the phase at $t=\frac{3}{2} T_{R F}$ (i.e. after the second gradient pulse) can be seen to be

$$
\begin{aligned}
\phi\left(z, \frac{3}{2} T_{R F}\right) & =\gamma\left(z-z_{c}\right)\left[-\int_{0}^{\frac{3}{2} T_{R F}} G_{z}\left(t^{\prime}\right) d t^{\prime}+G_{z} \frac{T_{R F}}{2}\right]+\frac{\pi}{2} . \\
& =\gamma\left(z-z_{c}\right)\left[-\int_{0}^{T_{R F}} G_{z}\left(t^{\prime}\right) d t^{\prime}-\int_{T_{R F}}^{\frac{3}{2} T_{R F}} G_{z}\left(t^{\prime}\right) d t^{\prime}+G_{z} \frac{T_{R F}}{2}\right]+\frac{\pi}{2} \\
& =\gamma G_{z}\left(z-z_{c}\right)\left[-T_{R F}+\frac{3}{2} T_{R F}-T_{R F}+\frac{T_{R F}}{2}\right]+\frac{\pi}{2} \\
& =\frac{\pi}{2} .
\end{aligned}
$$

In other words, the phase for all spins in the on-resonance rotating frame after the slice selection process, including the refocussing gradient pulse, is $\pi / 2$, the desired result.

\subsubsection{Spin Echo Sequence}

A spin echo (SE) is acquired using a minimum of two RF pulses. First, the MR signal must be re-oriented into the transverse plane, so a $\pi / 2$ pulse is applied. After a time delay, $t_{d}$, a $\pi$ pulse is applied in the $y^{\prime}$-direction to refocus the dephased spins back towards their original position in the transverse plane. $A \pi$ pulse in this context is known as a refocusing pulse. The full refocusing occurs after a total time of $2 t_{d}$. This is demonstrated in Fig. 2.5 

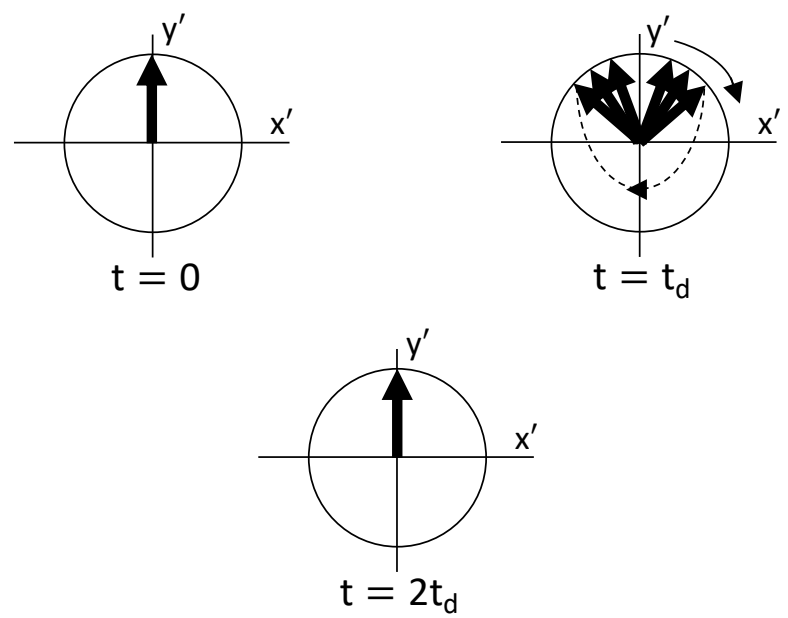

Figure 2.5: An example of the magnetization during a spin echo sequence in the $x^{\prime}-y^{\prime}$ plane. In this example of all the nuclear spins are slightly off resonance with respect to the rotating frame.

in the rotating reference frame. The fully refocussed magnetization at time $2 t_{d}$ is referred to as a spin echo. The time required to create this echo (i.e. $2 t_{d}$ ) is known as the echo time $\left(T_{E}\right)$. The $\pi$ pulses can be applied multiple times to generate further spin echoes as long as the $T_{2}$ of the sample is long enough. This is the basis of the turbo spin echo (TSE) sequence which can be used to acquire spin echo images more quickly since each of these echoes will correspond to a line of k-space.

At a time following the echo, or the final echo acquired in a TSE sequence, the transverse signal will decay completely, and magnetization will again solely relax back to the longitudinal direction. Once the magnetization achieves a value close to its equilibrium value, a $\pi / 2$ pulse is again applied and the application of $\pi$ pulses is repeated to fill more lines of $k$-space. The time between $\pi / 2$ RF pulses is known as the repetition time $\left(T_{R}\right)$. 


\subsubsection{Gradient Echo Sequence}

A gradient echo (GRE) is produced by using magnetic field gradients to dephase and rephase the transverse magnetization to produce one or more echoes. The contribution to the total phase in the on-resonance rotating reference frame due to a gradient pulse of duration $t_{G}$ can be determined by (see Section 2.3.3)

$$
\phi(r, t)=-v \int_{0}^{t_{G}}(\mathbf{G} \cdot r) d t^{\prime}
$$

where $t \geq t_{G}$. From this equation it can be seen that the application of a gradient pulse inherently induces dephasing of the MR signal since spins at different locations will have different phases. This dephasing can be completely reversed by applying a gradient pulse opposite in polarity and equal in magnitude and duration to the initial gradient. An example of a GRE acquisition is shown in Fig. 2.6. The phase after the second applied gradient is given by

$$
\phi(r, t)=-\nu \int_{0}^{t_{G}}(\mathbf{G} \cdot \mathbf{r}) \mathrm{dt}^{\prime}-\gamma \int_{\mathrm{t}_{\mathrm{G}}}^{\mathrm{t}}(-\mathbf{G} \cdot \mathbf{r}) \mathrm{dt}^{\prime}
$$

where $t \geq 2 t_{G}$. Eq. 2.57 indicates that after a time $2 t_{G}$, the phase has been refocused so that $\phi=0$ and a GRE has formed. Unlike a SE sequence, the rate of decay of the GRE signal is dictated by $T_{2}^{*}$ rather than $T_{2}$. This indicates that $\mathbf{B}_{\mathbf{0}}$ inhomogeneities don't allow for complete rephasing of the signal. Similar to the SE sequence, multiple gradient echoes can be generated by using more matched gradient pulse pairs. These additional echoes can be used to acquire data at a significantly faster rate; however, this is limited by the $T_{2}^{*}$ of the imaged object and the slew rate of the applied gradients. 


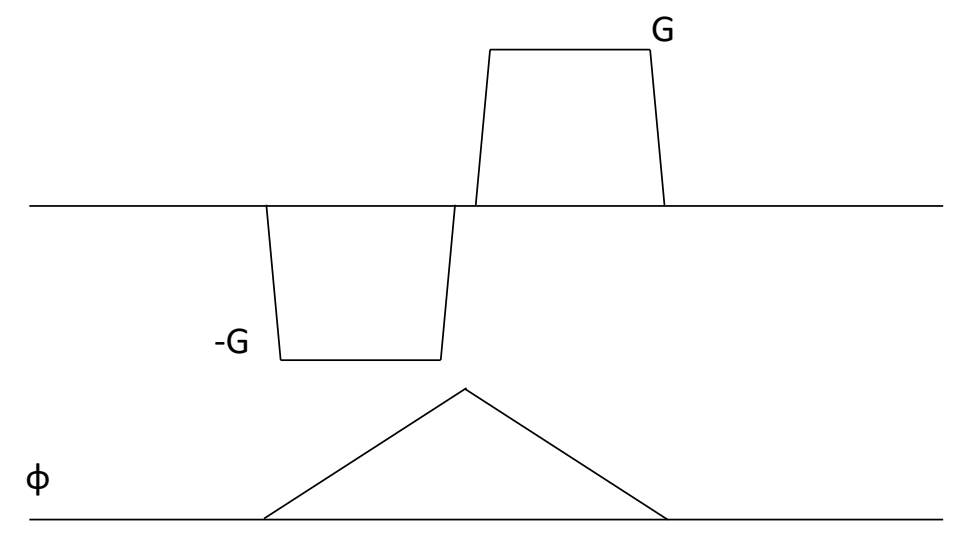

Figure 2.6: An example of a GRE sequence. This figure shows the pair of gradients of opposite polarity required to generate the desired phase evolution.

\subsubsection{Fast Low Angle Shot}

The Fast Low Angle SHot (FLASH) sequence is a GRE sequence that is designed to quickly measure changes in magnetization due to its use of small $\alpha$, spoiler gradients, and very short $T_{R}$ [32]. An MR sequence diagram for a conventional FLASH acquisition is shown in Fig. 2.7. The initial RF pulse tips the magnetization by an angle $\alpha$ such that for a time $t=\varepsilon$ immediately after the pulse, the components of the magnetization are given by

$$
M_{z}(\varepsilon)=M_{z}(0) \cos \alpha
$$

and

$$
M_{x y}(\varepsilon)=M_{z}(0) \sin \alpha
$$

Combining these with Eqs. 2.26 and 2.27, the components of the magnetization at the start of the next RF pulse $\left(t=T_{R}\right)$ in the laboratory frame are given by [27]

$$
M_{z}\left(T_{R}\right)=M_{0}\left(1-e^{-T_{R} / T_{1}}\right)+M_{z}(0) \cos \alpha e^{-T_{R} / T_{1}}
$$

and 


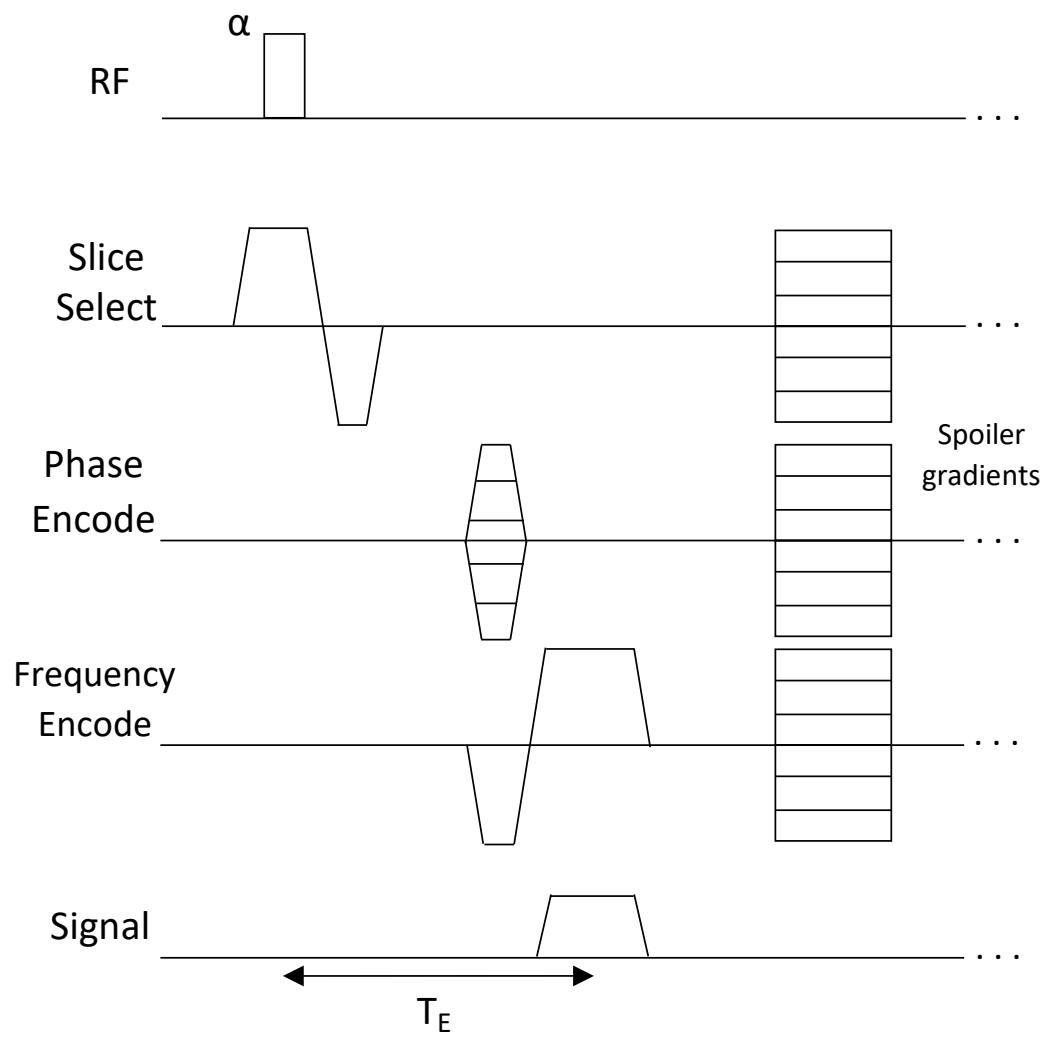

Figure 2.7: Example of the FLASH sequence. The GRE is generated by the first two gradients in the frequency encoding direction (see also Fig. 2.4). The rest of the gradient pulses in this sequence (other than the spoilers) are used to encode spatial information into the signal. The squares with the horizontal lines indicate gradient pulses where the amplitude is different each time the sequence is repeated.

$$
M_{x y}\left(T_{R}\right)=M_{z}(0) \sin \alpha e^{-T_{R} / T_{2}} e^{-i \omega_{0} t}
$$

As mentioned, an advantage of using FLASH is its speed. The ability to use a short $T_{R}$ is due to the use of spoiler gradients, which eliminates all remaining signal in the x-y plane. Once data acquisition from the initial RF pulse has concluded (i.e. filling a line of k-space), spoiling gradients are applied in all three gradient directions to quickly dephase the remaining magnetization in the transverse plane. In the rotating frame, the components of the magnetization before the application of the next RF pulse are, 


$$
M_{z^{\prime}}^{\prime}\left(T_{R}\right)=M_{0}\left(1-e^{-T_{R} / T_{1}}\right)+M_{z}(0) \cos \alpha e^{-T_{R} / T_{1}}
$$

and

$$
M_{x^{\prime} y^{\prime}}^{\prime}\left(T_{R}\right)=0
$$

where $\mathrm{M}_{\mathrm{z}^{\prime}}^{\prime}$ and $\mathrm{M}_{\mathrm{x}^{\prime} \mathrm{y}^{\prime}}^{\prime}$ indicate the magnetizations in the rotating frame before the second RF pulse. Note that the longitudinal magnetization is not affected by the application of the spoiler gradients and can be considered to be in a steady state, such that $\mathrm{M}_{\mathrm{z}^{\prime}}^{\prime}\left(\mathrm{T}_{\mathrm{R}}\right)=$ $\mathrm{M}_{\mathrm{z}}(0)$

Rearranging Eq. 2.62, this steady state magnetization $\left(\mathrm{M}_{\mathrm{z}, \mathrm{ss}}\right)$ can be expressed as

$$
M_{z, s s}=\frac{M_{0}\left(1-e^{-T_{R} / T_{1}}\right)}{1-\cos \alpha e^{-T_{R} / T_{1}}}
$$

As mentioned in Section 2.3.2, the GRE signal intensity follows a $\mathrm{T}_{2}^{*}$ decay, so the complex signal measured from the magnetization of the GRE generated at time, $t=T_{E}$, is given by

$$
S I_{G R E}=\rho M_{z, S S} \sin \alpha e^{-T_{E} / T_{2}^{*}} e^{\omega_{0} T_{E}}
$$

where $\rho$ is a proportionality constant determined primarily by the MRI scanner hardware. Therefore, the total measured signal for a FLASH sequence is obtained by combining Eqs. 2.64 and 2.65 to get:

$$
\mathrm{SI}_{\mathrm{FLASH}}\left(\mathrm{M}_{0}, T_{R}, T_{1}, \alpha, T_{E}, T_{2}^{*}\right)=\rho \frac{M_{0}\left(1-e^{-T_{R} / T_{1}}\right)}{1-\cos \alpha e^{-T_{R} / T_{1}}} \sin \alpha e^{-T_{E} / T_{2}^{*}} e^{\omega_{0} T_{E}} .
$$

Typically, only the magnitude of this signal is displayed or used in analysis. The intensity of the acquired signal is greatest when $\alpha$ is

$$
\alpha_{\mathrm{E}}=\arccos \left(\mathrm{e}^{-\mathrm{T}_{\mathrm{R}} / \mathrm{T}_{1}}\right)
$$

where $\alpha_{E}$ is known as the Ernst angle [30]. 


\subsubsection{Inversion Recovery $\mathrm{T}_{1}$ Sequences}

One important requirement for clinical imaging is the generation of contrast between tissues. Some sequences take advantage of the fact that different tissues have different $T_{1}$ values. A method to generate the greatest $T_{1}$ contrast is known as inversion recovery (IR). An example of an IR-SE sequence is shown in Fig. 2.8. Note that the first $\pi$ pulse in this sequence is an inversion pulse whereas the second one is a refocusing pulse. The refocusing pulse has no effect on the longitudinal magnetization (as long as $T_{E} \ll T_{1}$ ). The $\pi / 2$ pulse and the refocusing pulse work together to generate a spin echo. If the magnetization during the IR process in the rotating reference frame is $M_{z^{\prime}}$, and the initial longitudinal magnetization is $M_{0}$, then before any RF pulses are applied, $M_{z^{\prime}}=M_{0}$. Following an initial, perfect $\pi$ RF pulse,

$$
\mathrm{M}_{\mathrm{z}^{\prime}}=-\mathrm{M}_{0}
$$

The time at which the $\pi / 2$ RF pulse is applied is known as the inversion time (TI). The longitudinal magnetization at $\mathrm{t}=\mathrm{TI}$, from Eq. 2.28 , is then

$$
M_{z^{\prime}}(T I)=M_{0}\left(1-e^{-T I / T_{1}}\right)-M_{0} e^{-T^{-} / T_{1}}
$$

which gives

$$
M_{z^{\prime}}(T I)=M_{0}\left(1-2 e^{-T I / T_{1}}\right)
$$

The $\pi / 2$ pulse reorients the longitudinal magnetization into the transverse plane. Note that the refocusing $\pi$ pulse will then generate a SE but it will have no effect on $M_{z^{\prime}}$, as long as $T_{E} \ll T_{1}$, which is normally the case. Following the $\pi / 2$ pulse the longitudinal magnetization will relax back to its equilibrium value starting from $M_{z^{\prime}}=0$. Therefore, at a time $t=T_{R}$, the longitudinal magnetization becomes 

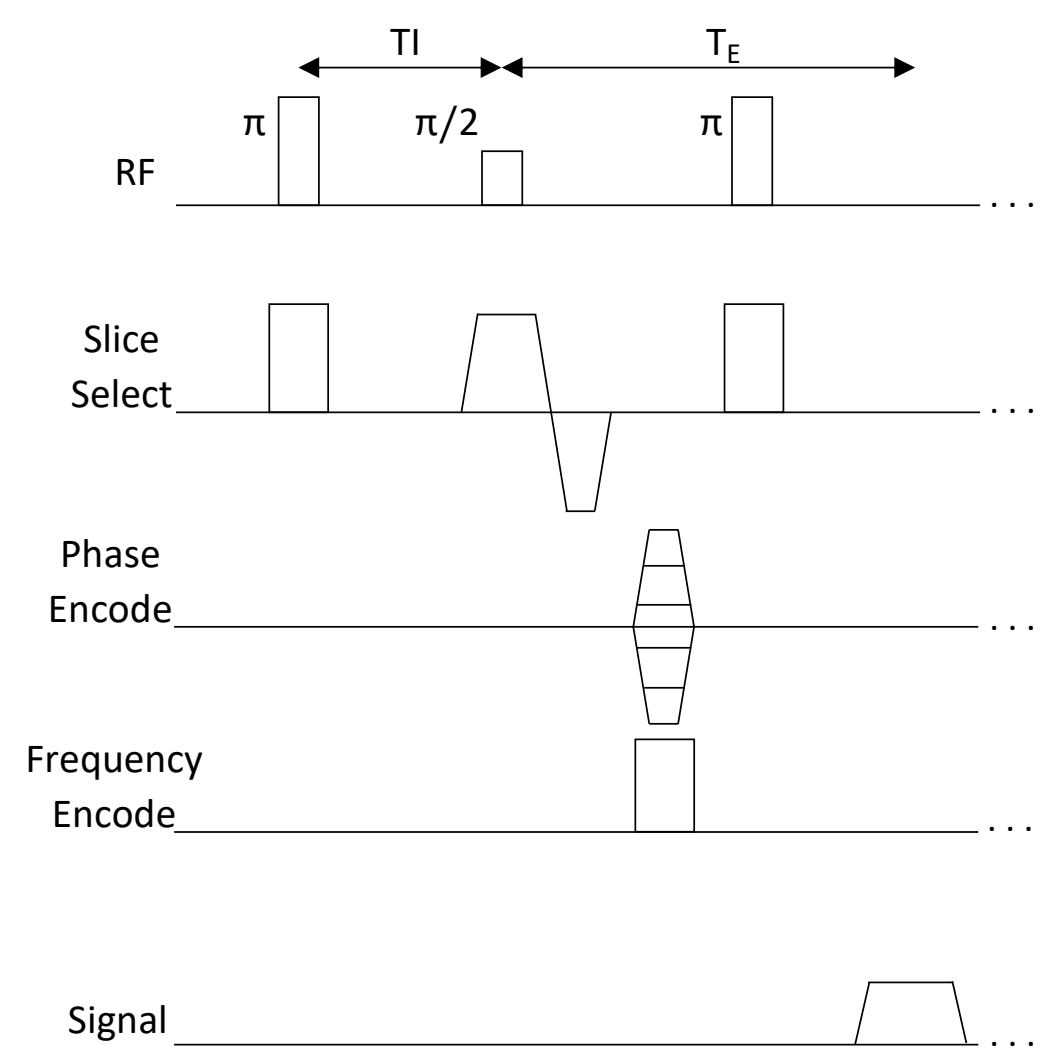

Figure 2.8: Example of a spin echo inversion recovery sequence.

$$
M_{z^{\prime}}\left(T_{R}\right)=M_{0}\left(1-e^{-\left(T_{R}-T I\right) / T_{1}}\right)
$$

It is desirable to have $\left(T_{R}-T I\right) \gg T_{1}$ so that the second term in Eq. 2.71 becomes negligible and the magnetization has fully relaxed back to its equilibrium value, $M_{0}$, before the next repetition of the sequence. However, in practice this is often impractical for IR imaging sequences. When this condition is not met the longitudinal magnetization at the beginning of a repetition period is not $M_{0}$ but rather it is equal to $M_{z^{\prime}}\left(T_{R}\right)$ from the previous repetition. Thus, immediately after the inversion pulse for the second repetition block

$$
M_{z^{\prime}}(\varepsilon)=-M_{0}\left(1-e^{-\left(T_{R}-T_{I}\right) / T_{1}}\right)
$$


so that

$$
\begin{aligned}
M_{z^{\prime}}(t) & =M_{0}\left(1-e^{-t / T_{1}}\right)-M_{0}\left(1-e^{-\left(T_{R}-T I\right) / T_{1}}\right) e^{-t / T_{1}} \\
& =M_{0}\left(1-2 e^{-t / T_{1}}+e^{-\left(t+T_{R}-T I\right) / T_{1}}\right) .
\end{aligned}
$$

Therefore, at $\mathrm{t}=\mathrm{TI}$ in this second repetition period, $\mathrm{M}_{\mathrm{z}^{\prime}}$ is given by

$$
M_{z^{\prime}}(T I)=M_{0}\left(1-2 e^{-T I / T_{1}}+e^{-T_{R} / T_{1}}\right)
$$

This will also be true for all subsequent $T_{R}$ periods.

The signal of interest in an IR sequence, $S_{I R}$, is the signal following the $\pi / 2$ pulse. Thus, from Eq. 2.74,

$$
S I_{I R}=\rho M_{0}\left(1-2 e^{-T I / T_{1}}+e^{-T_{R} / T_{1}}\right),
$$

where $\rho$ is a proportionality constant. Rather than measuring the signal induced by $\mathrm{M}_{\mathrm{z}^{\prime}}(\mathrm{TI})$ directly, a SE is generated, and it is this echo signal that is actually measured. Even so, if $T_{E}$ is short, Eq. 2.75 describes the signal measured with an IR sequence quite well.

For the work reported in this thesis the spatial encoding for the IR measurements was done using a single shot echo planar imaging (EPI) approach. An example of an EPIIR sequence is shown in Fig. 2.9. With single shot EPI-IR, the IR part of the sequence is executed only once so that $T_{R}$ is effectively infinite. For this case, the magnetization that generates the signal is given by Eq. 2.70 so that, in this case

$$
S I_{\mathbb{R}}=\rho M_{0}\left(1-2 e^{-T I / T_{1}}\right)
$$

This equation is of the form

$$
\mathrm{SI}=\mathrm{A}-\mathrm{B} \mathrm{e}^{-\mathrm{TI} / \mathrm{T}_{1}}
$$

where $A$ represents the signal at equilibrium and $B=2 A$, in the ideal case. In practice, it is very difficult to generate a $\pi$ pulse that will satisfy Eq. 2.68 perfectly. If the 

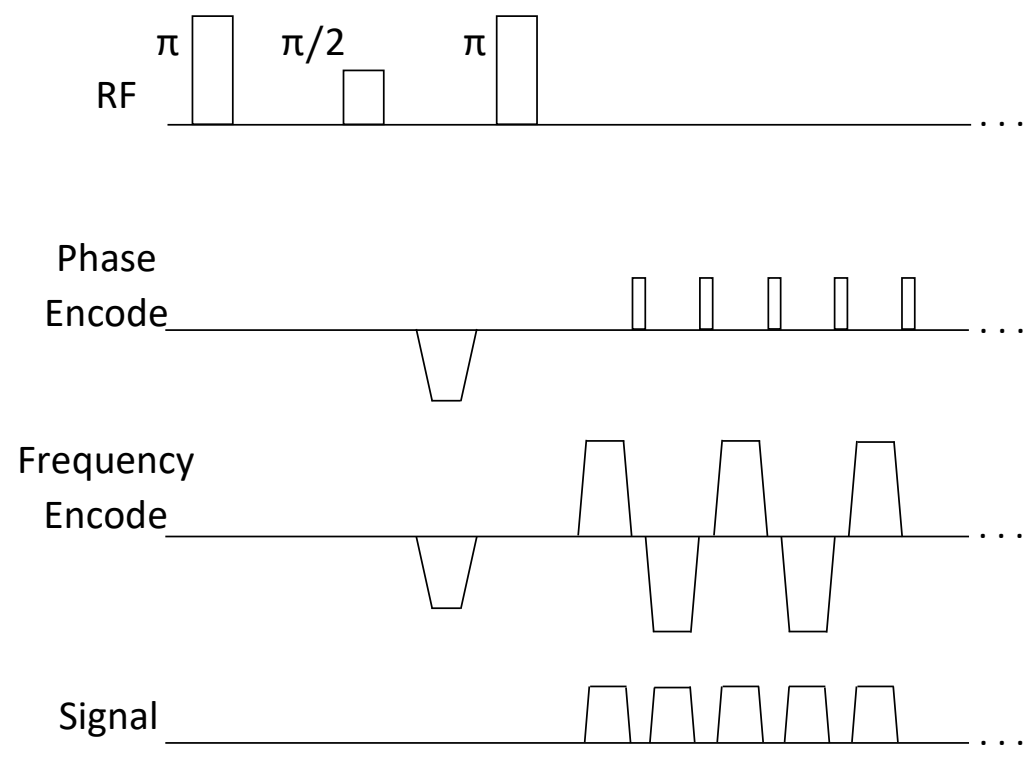

Figure 2.9: An example of an EPI-IR sequence diagram.

magnetization after the inversion pulse is given by $-M_{z^{\prime}}$ where $M_{z^{\prime}}<M_{0}$, then Eq. 2.70 becomes

$$
M_{z^{\prime}}(T I)=M_{0}-\left(M_{0}+M_{z^{\prime}}\right) e^{-T I / T_{1}}
$$

The signal generated from this magnetization will still be of the form given by Eq. 2.77 but, in this case, $B<2 A$. If an EPI-IR sequence is performed with multiple $\mathrm{TI}$ values, a relaxation curve can be created and the parameters $A, B$, and $T_{1}$ can easily be obtained using standard curve fitting techniques.

\subsubsection{Look-Locker IR Sequences}

While EPI-IR can be used to accurately measure $T_{1}$, it is a relatively slow technique. EPI-IR relies on the full relaxation of $M_{z}$ back to equilibrium before another $\mathrm{TI}$ value can be 
measured. A method was developed by Look and Locker [33] to circumvent this delay, while maintaining $T_{1}$ accuracy. Following an initial $\pi$ RF pulse, a series of $n$ small- $\alpha$ RF pulses are applied at times $\mathrm{n} \cdot \mathrm{TI}$, where $\mathrm{n}$ is an integer greater than zero. The RF pulses must be small enough that they perturb the $T_{1}$ regrowth minimally but, at the same time, provide a large enough transverse magnetization to give an acceptable signal intensity. With this approach, multiple points on the relaxation curve can be measured in one repetition of the sequence. The signal intensity at each $\mathrm{Tl}$ is given by an equation of the form

$$
S I(n \cdot T I)=A-B e^{-n \cdot T I / T_{1}^{*}}
$$

where $T_{1}^{*}$ is the effective relaxation time. Due to the multiple perturbations of the relaxation caused by the $\alpha$-pulses, $T_{1}^{*}$ will, in general, differ from the true $T_{1}$ but the two are related by

$$
\mathrm{T}_{1}=\mathrm{T}_{1}^{*}(\mathrm{~B} / \mathrm{A}-1)
$$

when $\alpha$ is small and $T_{R} \ll T_{1}^{*}<T_{1}[32]$.

The LL method has been adjusted to acquire $T_{1}$ data in cardiac sequences in a single breath-hold. This method, known as Modified Look-Locker Inversion Recovery (MOLLI), is designed to obtain data at specific points in the cardiac cycle, over multiple cycles, and combine data from multiple $\alpha$-pulses to form a single apparent relaxation curve [34] (see Fig. 2.10). This single curve is then used to determine the true $T_{1}$ of the data. While the modifications to the LL method are important for the acquisition of cardiac data, they have minimal added benefit for the $T_{1}$ data obtained in the brain.

After each $\alpha$-pulse of a LL sequence, the partially relaxed signal is measured by 
acquiring a full image using a fast imaging sequence. MOLLI uses what is known as a steady-state free precession (SSFP) sequence. SSFP refocuses transverse magnetization following the initial application of RF pulses and centered in time before the next RF pulse rather than spoiling it, like in a FLASH sequence. This has the added advantage of achieving a higher signal-to-noise ratio (SNR) and minimally affecting the $T_{1}$ relaxation curve [36].

The LL method has been used by several groups for studying $T_{1}$ relaxation in the brain [37-41]; however, MOLLI has not previously been used for brain studies. The MOLLI prototype sequence used for the work presented in this thesis was provided to The Ottawa Hospital by Siemens as part of a Works-in-Progress (WIP) package.

\subsection{MRI Contrast Enhancement}

The sequences described in the previous sections produce images based on the measured MR signal converted to grayscale data. Depending on the sequence used, a certain tissue may be darker or brighter compared to another and when multiple tissue types are imaged together, they can be differentiated based on the signal contrast. Certain tumour tissues, regardless of the MR sequence used, may not achieve enough contrast between themselves and normal healthy tissue to be identified with clinical imaging sequences. In order to produce a greater tumour tissue contrast, a material known as a contrast agent can be used.

A gadolinium-based contrast agent (GBCA) is the most common type of contrast agent used in MRI. GBCAs contain a ligand that chelates Gd ions. The ligand effectively 


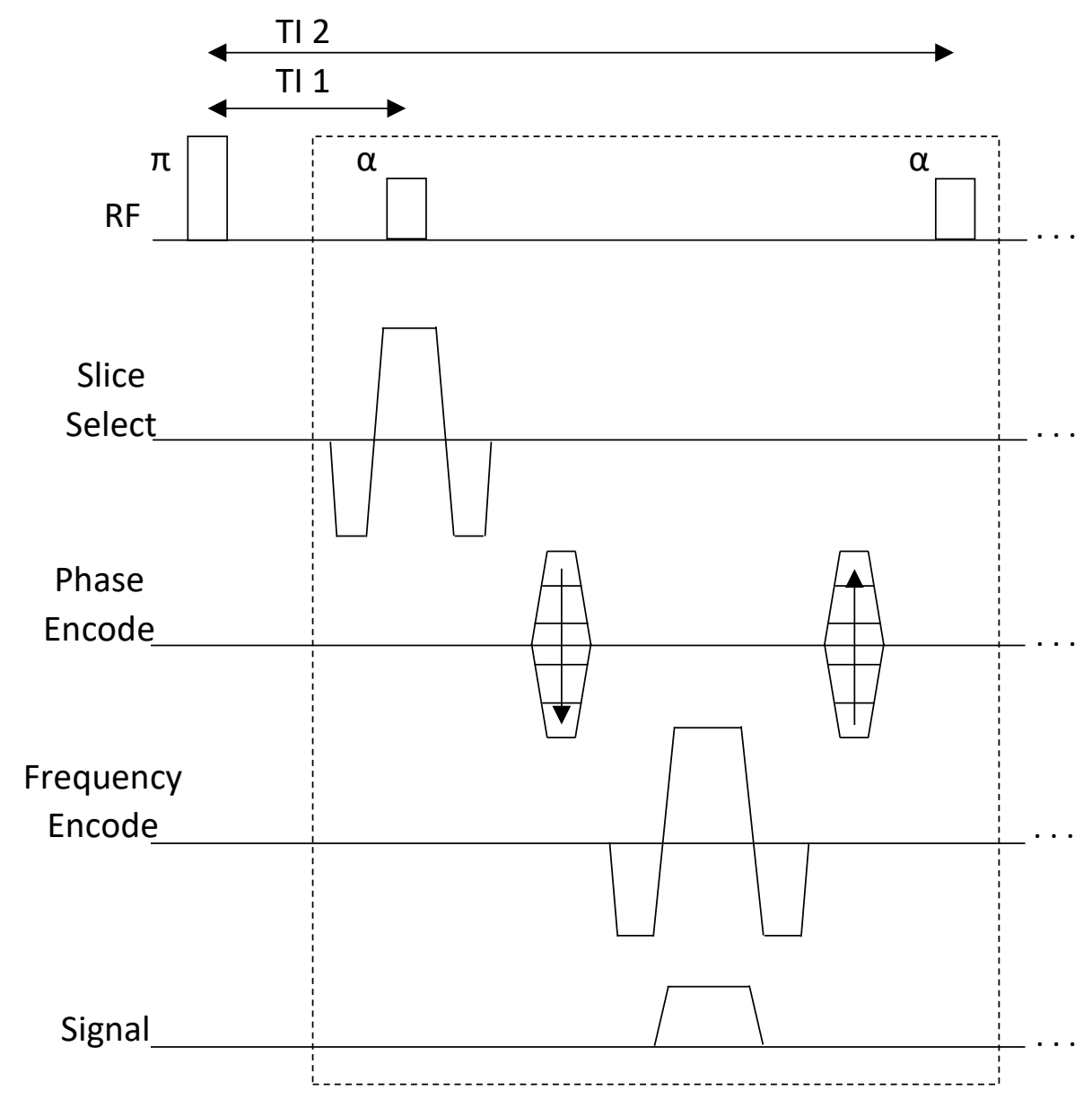

Figure 2.10: Example of a modified Look-Locker inversion recovery sequence. The part of the sequence within the dashed box corresponds to a SSFP sequence known as true Fast Imaging with Steady-state Precision (trueFISP) [35]. The trueFISP readout is repeated for as many TI values as desired.

'cages' the ion and prevents the highly toxic Gd ions from entering the bloodstream. This work used a GBCA with the gadobutrol molecule (Gd-DO3A-butrol) and is commercially produced with the name Gadovist ${ }^{\circledR}$ 1.0 M (Bayer HealthCare Pharmaceuticals, Toronto, Ontario, Canada). Gadobutrol is a paramagnetic GBCA that utilizes a macrocyclic structure to house a $\mathrm{Gd}^{3+}$ ion.

The $\mathrm{Gd}^{3+}$ ion is highly desirable to use as the acting factor in an MR contrast agent 
due to its unique electron structure, containing seven unpaired electrons, which allows for the efficient altering of the local magnetic field [42]. This electronic structure creates inhomogeneities in the magnetic field in the vicinity of the $\mathrm{Gd}^{3+}$ ion to selectively affect the MR signal and establish greater tissue contrast in regions with higher blood flow (e.g. tumours versus healthy tissue). These effects are time-dependent as the motion of the gadobutrol molecules continuously changes the bulk magnetic moment of the tissues affected by these molecules.

The change in magnetic field is due to the changing magnetic susceptibility of the tissues induced by the presence of the GBCA. Magnetic susceptibility, $\chi$, describes a material's ability to become magnetized in an external magnetic field and is the proportionality constant between the magnetization of an object $(\mathbf{M})$ and the magnetic field intensity $(\mathbf{H})$, given as

$$
\mathbf{M}=\chi \mathbf{H}
$$

The total magnetic field at a location inside a material is proportional to $\mathbf{H}$ and is given by

$$
\mathbf{B}=\mu_{0}(1+\chi) \mathbf{H}
$$

where $\mu_{0}$ is the permeability of free space $\left(\mu_{0}=4 \pi \times 10^{-7} \mathrm{~T} \mathrm{~m} \mathrm{~A}^{-1}\right)$. Additionally, the magnetic field can be expressed in terms of the magnetization by combining Eqs. 2.81 and 2.82 to give

$$
\mathbf{B}=\mu_{0} \frac{1+\chi}{\chi} \mathbf{M} .
$$

Another consequence of the presence of a GBCA is the shortening of both $T_{1}$ and $\mathrm{T}_{2}$ relaxation times based on the GBCA's relaxivity, which describes the rate of relaxation for a particular [Gd]. The relationships between the relaxivity values for $T_{1}$ and $T_{2}$ 
(denoted as $r_{1}$ and $r_{2}$, respectively) and [Gd] are given by:

$$
\frac{1}{T_{1}}=\frac{1}{T_{1,0}}+r_{1}[G d]
$$

and

$$
\frac{1}{T_{2}}=\frac{1}{T_{2,0}}+r_{2}[G d]
$$

where $T_{1,0}$ and $T_{2,0}$ are the relaxation terms when no GBCA is present. Equations 2.84 and 2.85 show that for one or more measured $T_{1}$ or $T_{2}$ values for given [Gd] values, the relaxivity of the [Gd] can be computed. Relaxivity values depend on the strength of the main magnetic field, the type of medium containing the GBCA, and the medium temperature $[43,44]$. The stated $r_{1}$ and $r_{2}$ values for gadobutrol in human plasma at body temperature at 3 T are $4.1 \pm 0.2 \mathrm{mM}^{-1} \mathrm{~s}^{-1}$ and $5.4 \pm 0.5 \mathrm{mM}^{-1} \mathrm{~s}^{-1}$, respectively [43]. For room-temperature water at $3 \mathrm{~T}, \mathrm{r}_{1}=4.7 \pm 0.2 \mathrm{mM}^{-1} \mathrm{~s}^{-1}$ and $\mathrm{r}_{2}=5.8 \pm 0.3 \mathrm{mM}^{-1} \mathrm{~s}^{-1}$ [43]. The values of $T_{1,0}$ for room-temperature water and human plasma at $37^{\circ} \mathrm{C}$ are $2591 \pm 45 \mathrm{~ms}$ and $3070 \pm 15 \mathrm{~ms}$, respectively. The corresponding values of $\mathrm{T}_{2,0}$ are $959 \pm$ $27 \mathrm{~ms}$ and $452 \pm 5 \mathrm{~ms}[43]$.

\subsubsection{Magnetic Susceptibility for an Infinite Cylinder}

The effect of altering the local magnetic field in a tissue due to the use of a GBCA results in a susceptibility effect in the resulting image which is geometry-dependent. For this work, MR phase was measured in the superior sagittal sinus (SSS), which can be approximated as a long, straight cylindrical vessel at the point of measurement. The susceptibility effect can be determined numerically for this shape both inside and outside 
of the cylinder. A schematic of this scenario is shown in Fig. 2.11 where the cylinder's central axis is parallel to the z-axis. The cylinder is assumed to contain a material of constant susceptibility and to be surrounded by free space. If $\mathbf{B}_{\mathbf{0}}$ is at an angle with the zaxis such that

$$
\mathbf{B}_{\mathbf{0}}=\mathrm{B}_{0}(\sin \theta \hat{\mathbf{x}}+\cos \theta \hat{\mathbf{z}})
$$

then the magnetic field at a point outside the cylinder, at a distance $\xi$ away from the central axis of the cylinder is given by [30]

$$
\mathbf{B}_{\text {out }}=B_{0} \cos \theta \hat{\mathbf{z}}+B_{0} \sin \theta\left[\hat{\mathbf{x}}+\frac{\chi / 2}{1+\chi / 2}\left(\frac{r}{\xi}\right)^{2} \cdot(\cos 2 \varphi \hat{\mathbf{x}}+\sin 2 \varphi \hat{\mathbf{y}})\right],
$$

and the magnetic field inside the cylinder is [30]

$$
\mathbf{B}_{\text {in }}=B_{0} \cos \theta(1+\chi) \hat{\mathbf{z}}+\frac{1+\chi}{1+\chi / 2} B_{0} \sin \theta \hat{\mathbf{x}}
$$

It is important to note that it is not necessarily the magnetic field itself in the SSS that is relevant, but the difference of magnetic field $(\Delta B)$ between the external $B_{0}$ field and the field due to the presence of the exogenous GBCA. As most GBCAs are paramagnetic, the limit where $\chi \ll 1$ can be used so that $\Delta B$ outside and inside the vessel can be found to be [30]:

$$
\begin{aligned}
\Delta \mathrm{B}_{\text {out }} & \simeq \frac{\chi}{2} \mathrm{~B}_{0} \sin ^{2} \theta\left(\frac{\mathrm{r}}{\xi}\right)^{2} \cos 2 \varphi \\
\Delta \mathrm{B}_{\text {in }} & \simeq \frac{\chi}{2} \mathrm{~B}_{0}\left(1+\cos ^{2} \theta\right),
\end{aligned}
$$

where the properties $\Delta \mathbf{B}=\mathbf{B}-\mathbf{B}_{\mathbf{0}}$ and $\Delta \mathrm{B}=\Delta \mathbf{B} \cdot \mathbf{B}_{\mathbf{0}}$ were used in both cases.

The derivation of these equations is based on a model which assumes that there is a continuous distribution of magnetic sources inside the cylinder. When the distance 


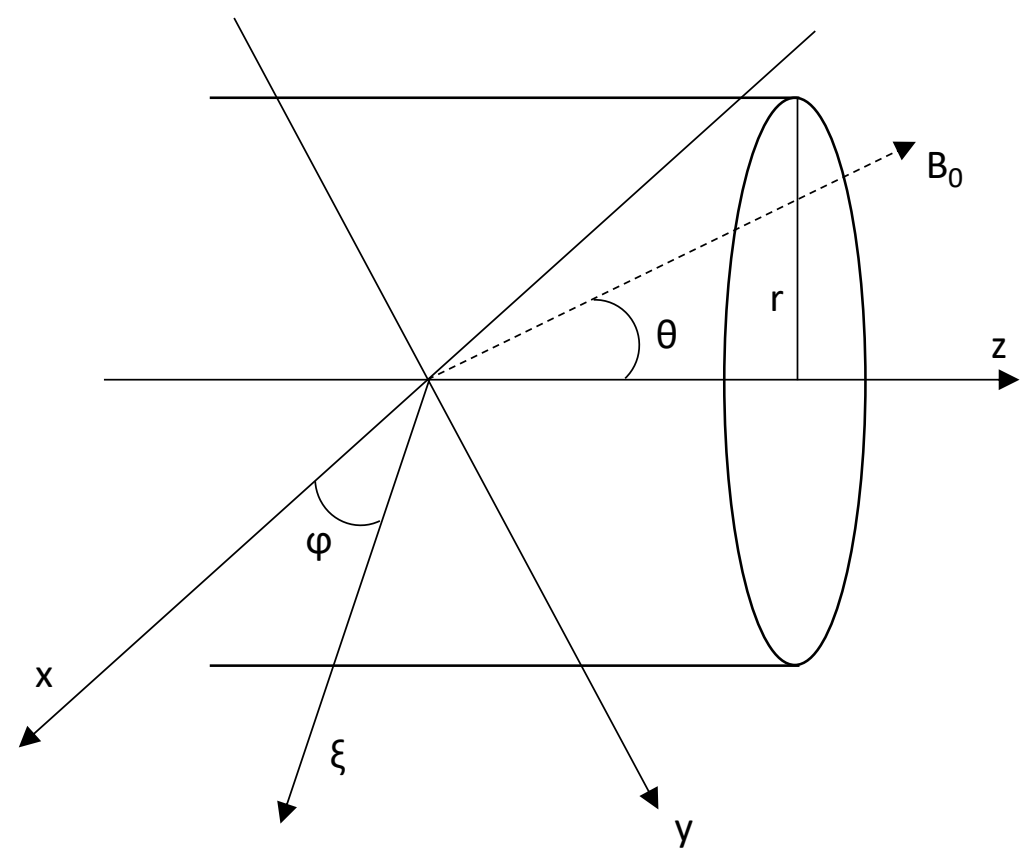

Figure 2.11: Schematic for the calculation of magnetic field outside and inside of an infinitely long cylinder with radius $r$.

between the position of interest and the magnetic field source is large, the assumption of a continuous distribution of sources is reasonable; however, this model breaks down inside the cylinder where neighbouring magnetic dipoles need to be considered. Furthermore, for the situation of interest here, the cylinder corresponds to a vein which is surrounded by tissue (not free space) with a non-zero susceptibility, $\chi_{e}$.

When corrections for these two considerations are applied, it can be shown [30] that $\Delta B_{i n, l o c a l}$, the change from the applied field to the local magnetic field experienced by the nuclear magnetic moments inside the cylinder (i.e. the vein) is given by

$$
\Delta B_{\text {in, local }}=\frac{1}{2} \Delta \chi B_{0}\left(\cos ^{2} \theta-\frac{1}{3}\right)+\frac{1}{3} \chi_{e} B_{0}
$$

where $\Delta \chi \equiv \chi_{i}-\chi_{e}$ and $\chi_{i}$ is the susceptibility inside the cylinder. It is assumed here that 
$x_{\mathrm{i}} \ll 1$ and $\chi_{\mathrm{e}} \ll 1$. In a similar way, it can be shown that when these corrections are applied, the corresponding change in magnetic field for spins outside the vessel, $\Delta \mathrm{B}_{\text {out,local }}$, is given by

$$
\Delta \mathrm{B}_{\text {out }, \text { local }}=\frac{\Delta \chi}{2} \mathrm{~B}_{0} \sin ^{2} \theta\left(\frac{\mathrm{r}}{\xi}\right)^{2} \cos 2 \varphi+\frac{1}{3} \chi_{\mathrm{e}} \mathrm{B}_{0} .
$$

Normally, the susceptibility inside the cylinder will be that of blood, $\chi_{0}$, but when a GBCA is present, the total susceptibility inside the cylinder becomes [45]

$$
\chi_{i}=\chi_{0}+\chi_{m}[G d]
$$

where $\chi_{m}$ is the molar susceptibility of the GBCA $\left(3.4 \times 10^{-4} \mathrm{M}^{-1}[46]\right)$ and [Gd] is the concentration of the GBCA. For many applications, the difference between $\chi_{\mathrm{e}}$ and $\chi_{0}$ can be considered to be negligible and the assumption that $\chi_{e}=\chi_{0}$ is often used. However, since the magnetic susceptibility of blood depends on the hematocrit and oxygenation level of the blood [30], this assumption will not be used here.

It is useful to define $\delta \chi$ to be the value of $\Delta x$ when $[G d]=0$. Then

$$
\delta \chi=\chi_{0}-\chi_{e}
$$

and

$$
\Delta \chi=\chi_{\mathrm{m}}[\mathrm{Gd}]+\delta \chi
$$

Inserting Eqs. 2.94 and 2.95 into Eq. 2.91 gives

$$
\begin{aligned}
\Delta \mathrm{B}_{\text {in,local }} & =\frac{1}{2}\left(\chi_{m}[G d]+\delta \chi\right) B_{0}\left(\cos ^{2} \theta-\frac{1}{3}\right)+\frac{1}{3}\left(\chi_{0}-\delta \chi\right) B_{0} \\
& =\frac{1}{3} \chi_{o} B_{0}+\frac{1}{2} \chi_{m}[G d] B_{0}\left(\cos ^{2} \theta-\frac{1}{3}\right)+\frac{1}{2} \delta \chi B_{0}\left(\cos ^{2} \theta-1\right) .
\end{aligned}
$$




\subsubsection{Arterial Input Function}

It is useful to identify here some key aspects of the AIF. The AIF reflects the passage of an injected GBCA as it passes through a volume of interest. The AIF has three main components, as shown in Fig. 2.12: the baseline (before injection of the GBCA; left shaded column), the passage of the GBCA (including the AIF peak; the white section), and the washout (when the $[G d]$ reaches an equilibrium value; right shaded column). $T_{1}$ values for the baseline and washout will be referred to as $T_{1,0}$ and $T_{1, w}$, respectively. Measured $T_{1,0}$ and $T_{1, w}$ values, along with a known $r_{1}$ value, can be used to compute [Gd] (see Eq. 2.84); however, $T_{1}$ measurements are typically too time-consuming to be a feasible method for determining the full AIF. Changes to either the MR signal magnitude or phase as the bolus passes through the tissue of interest are normally used to obtain the AIF instead.

\subsubsection{GBCA Effect on MR Signal Magnitude and Phase}

Equation 2.66 demonstrates the measured SI from a FLASH sequence. When a GBCA is present, the $T_{1}$ value will depend on [Gd] (see Eq. 2.84) and the acquired SI will change accordingly. Starting from Eq. 2.66, the [Gd] can be determined directly from the SI data. For convenience, $E([G d])$ and $M_{0}^{\prime}$ will be defined as

$$
E([G d])=e^{-T_{R}\left(1 / T_{1,0}+r_{1}[G d]\right)} .
$$

and

$$
M_{0}^{\prime}=M_{0} \sin \alpha e^{-T_{E} / T_{2}^{*}}
$$

respectively. The signal magnitude, from Eq. 2.66 can be represented as: 


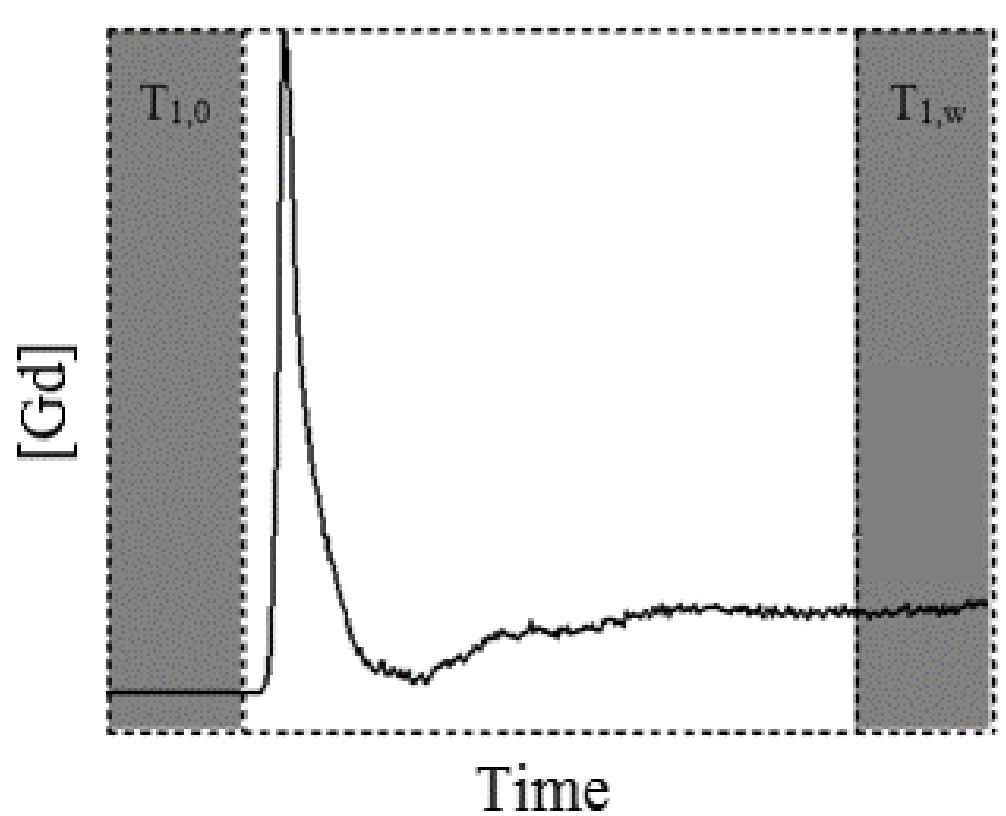

Figure 2.12: Example of an acquired AIF, which includes the AIF baseline (left shaded column), the main passage of the GBCA (white box) and the washout (right shaded column). This example was measured in a phantom.

$$
S I_{\mathrm{FLASH}}=\frac{1-\mathrm{E}([\mathrm{Gd}])}{1-\cos \alpha \mathrm{E}([\mathrm{Gd}])} \mathrm{M}_{0}^{\prime} \text {. }
$$

By rearranging Eq. 2.99 and substituting Eqs. 2.97 and 2.98 into it, the [Gd] as a function of measured signal intensity can be shown to be

$$
[\mathrm{Gd}](\mathrm{t})=\frac{1}{\mathrm{r}_{1}} \cdot\left\{-\frac{1}{\mathrm{~T}_{\mathrm{R}}} \cdot \ln \left[\frac{\mathrm{SI}_{\mathrm{FLASH}}-\mathrm{M}_{0}^{\prime}}{\mathrm{SI}_{\mathrm{FLASH}} \cdot \cos \alpha-\mathrm{M}_{0}^{\prime}}\right]-\frac{1}{\mathrm{~T}_{1,0}}\right\}
$$

The relationship between the intensity of the measured signal and [Gd] is non-linear and not intuitive; however, as will be shown below, there is a simple linear relationship between $[\mathrm{Gd}]$ and the phase of the measured signal.

From Eq. 2.34, the phase of the MR signal at a time $t=T_{E}$ is given by 


$$
\phi\left(r, T_{E}\right)=-\gamma \int_{0}^{T_{E}} B\left(r, t^{\prime}\right) d t^{\prime}
$$

As a bolus of GBCA passes through an artery or vein, $B(r, t)$ will change with time as [Gd] changes. This temporal dependence will be indicated here with the variable $\tau$. On the time scale of $T_{E}$ (i.e. a few $m s$ ), $B(r, t)$ can be considered to be time-independent and taken outside the integral in Eq. 2.101 which can now be written as

$$
\phi(r, \tau)=-\gamma B(r, \tau) T_{E}
$$

The total magnetic field, $\mathbf{B}(\mathbf{r}, \tau)$, is the sum of the external magnetic field and a contribution from the material in the magnet $\left(\mathbf{B}_{\mathbf{m}}\right)$ :

$$
B(r, t)=B_{0}+B_{m}(r, t) .
$$

If the artery or vein is taken to be an infinitely long cylinder, then $B_{m}(r, t)$ for positions inside the cylinder, is equal to $\Delta B_{\text {in,local }}$ given in Eq. 2.96:

$$
B_{m}(r, \tau)=\frac{1}{3} \chi_{0} B_{0}+\frac{1}{2} \chi_{m}[G d](\tau) B_{0}\left(\cos ^{2} \theta-\frac{1}{3}\right)+\frac{1}{2} \delta \chi B_{0}\left(\cos ^{2} \theta-1\right)
$$

where the notation $[G d](\tau)$ denotes the time-dependence of the concentration of the GBCA. For $\tau=0$, the bolus will not have arrived so that

$$
B_{m}(r, 0)=\frac{1}{3} \chi_{0} B_{0}+\frac{1}{2} \delta x B_{0}\left(\cos ^{2} \theta-1\right)
$$

and

$$
\phi_{0}(r)=\phi(r, 0)=-\frac{1}{3} \chi_{0} \omega_{0} T_{E}-\frac{1}{2} \delta \chi \omega_{0} T_{E}\left(\cos ^{2} \theta-1\right)
$$

When $[G d]>0$, the phase is given by

$$
\phi(r, \tau)=-\frac{1}{3} \chi_{0} \omega_{0} T_{E}-\frac{1}{2} \chi_{m}[G d](\tau) \omega_{0} T_{E}\left(\cos ^{2} \theta-\frac{1}{3}\right)-\frac{1}{2} \delta \chi \omega_{0} T_{E}\left(\cos ^{2} \theta-1\right) .
$$

The phase change can now be calculated as 


$$
\Delta \phi(\tau) \equiv \phi(r, \tau)-\phi_{0}(r)=-\frac{\omega_{0} T_{E}}{2} \chi_{m}[G d](\tau)\left(\cos ^{2} \theta-\frac{1}{3}\right)
$$

where the spatial dependence has been dropped since the GBCA is considered to be wellmixed inside the vessel such that [Gd] will be the same at all locations.

As [Gd] increases, the magnetic field experienced by the nuclear spins at this location also increases causing the spins to precess in the clockwise direction faster than the rotating frame. This explains the negative sign in the equation for $\Delta \phi(\tau)$. This sign is commonly dropped so that an increase in [Gd] results in an increased phase change (in the positive direction).

If $\Delta \phi(\tau)$ is measured over a period of time, then [Gd] can be calculated from [47]

$$
[\mathrm{Gd}](\tau)=\frac{-\Delta \phi(\tau)}{\omega_{0} \chi_{m} T_{E} f(\theta)}
$$

where

$$
f(\theta)=\frac{1}{2}\left[\cos ^{2} \theta-\frac{1}{3}\right]
$$

is the geometry factor for the infinite cylinder approximation.

It is important to note that $\delta x$ does not appear in Eq. 2.109. This means that the difference in magnetic susceptibility values between the extravascular space and the interior of the vessel, which is affected by the hematocrit and the oxygenation level of the blood, has no effect on the measured $[G d](\tau)$ values. It is also worth noting that the $\delta x$ term disappears from Eqs. 2.104-2.107 when $\theta=0$, which is the case for all of the experimental results presented in this thesis. 


\section{Chapter 3}

\section{Partial Volume Averaging and the Accuracy of the Arterial Input Function}

\subsection{Introduction}

The principles of Dynamic Contrast-Enhanced (DCE)-MRI and the Arterial Input Function (AIF) were briefly introduced in Sections 2.2.2 and 2.5.2. In the first part of this chapter, MRI techniques used for measuring the AIF will be reviewed. It is vital to have an accurately measured AIF to ensure that the pharmacokinetic parameter values obtained from the subsequent DCE-MRI analysis are as diagnostically reliable as possible. The primary focus of this chapter will be a careful assessment of how partial volume averaging (PVA) affects the accuracy of AIF measurements in MRI.

The effects of PVA on the real and imaginary components, the magnitude, and the phase $(\phi)$ of the MR signal are discussed in general terms in Section 3.3. The effect of PVA on phase accuracy is dealt with in considerable detail in Sections 3.4 and 3.5. The analysis shows that the phase error caused by PVA increases with the concentration of a 
gadolinium-based contrast agent (GBCA). In Chapter 4, a novel method is presented for the acquisition of the AIF which is more accurate than methods currently used clinically. The motivation for this method is the recognition that the experimental uncertainty for the phase at the baseline (i.e. the start of the AIF when the concentration of the GBCA; [Gd]) is considerably larger than it is for the washout (i.e. the end of the AIF when the [Gd] has reached equilibrium).

With DCE-MRI, a patient is given a bolus injection of a GBCA intravenously and the effect of this contrast agent on the MR signal as it passes through the tissues of interest is measured. The AIF is a function that characterizes the blood flow to the tissues that it supplies. As the bolus of GBCA passes through an artery, it has a strong effect on both the magnitude and phase of the MR signal where, accordingly, either can be used to measure the AIF. These two methods will be reviewed in Section 3.2, where it will be shown that using the phase is a superior approach.

One issue with both of these AIF measurement methods, when used for DCE-MRI of the brain, is that the cerebral arteries are very small. It is difficult to track the true changes to the MR signal caused by the GBCA without contamination from the MR signal of surrounding tissue (i.e. partial volume averaging, PVA). PVA will be discussed in detail in section 3.3.

The blood flow into a tissue, the AIF, is closely related to the blood flow leaving the tissue which is known as the venous output function (VOF). Since veins tend to be larger than the corresponding arteries, PVA is less of a problem when measuring the VOF. It is for this reason that the VOF is often used as a surrogate for the AIF. The ramifications 
of this substitution will be discussed in Section 3.2.

In Section 3.3, expressions are given for the magnitude and the phase of the MR signal when PVA is present. It can be seen from these equations that when the phase of the MR signal is the same inside the artery (or vein) as it is outside, the PVA has no effect on the measured phase. Unfortunately, this simple case does not apply to either AIF or VOF measurements. In Section 3.4, it will be shown that blood flow in the slice selection direction and a difference in the magnetic susceptibility of the venous blood compared to the surrounding tissue both cause a phase shift for the intravascular signal relative to the extravascular signal.

The results from Sections 3.2-3.4 will be combined in Section 3.5 where several examples, using realistic experimental parameter values, will be presented. This motivates a novel MR approach to the measurement of the AIF (or VOF) which significantly reduces the phase error associated with PVA. Since these phase errors have a direct effect on the concentration of the GBCA ([Gd]) values obtained from the phase measurements, it is important that these phase errors be as small as possible. Experimental validation of this new technique will be presented in Chapters 5 and 6.

\subsection{Background}

\subsubsection{Comparison of Signal Intensity Versus Signal Phase for AIF Measurement}

The MR signal is complex, such that when an acquisition is performed both real and imaginary parts of the signal are obtained simultaneously. From this, the signal intensity 
(SI) is computed (see Eq. 2.36) and this is what is commonly displayed in a typical MR image. The angle between the $x^{\prime}$-axis and the complex signal is $\phi$ (see Eq. 2.37 and Fig. 2.3). In Section 2.5.3, the change in both $\mathrm{SI}$ and $\phi$ as a function of changing [Gd] in time $([\mathrm{Gd}](\mathrm{t}))$ were derived in Eqs. 2.100 and 2.109, respectively. Based on their appearance, it seems that both functions are viable methods to measure $[\mathrm{Gd}](\mathrm{t})$. An evaluation of each of these two methods will be provided here.

$[G d](t)$ cannot be extracted simply from SI. It also requires knowledge of several properties of the sequence, such as the repetition time $\left(T_{R}\right)$, the flip angle $(\alpha)$, the echo time $\left(T_{E}\right)$, as well as knowledge about the object being imaged, such as its baseline $T_{1}$ value $\left(T_{1,0}\right)$, its longitudinal relaxivity value $\left(r_{1}\right)$, as well as its equilibrium magnetization value $\left(M_{0}\right)$. The $M_{0}$ of a sample is typically not known, so assumptions would have to be made in order to determine it accurately. One of the most significant issues when using SI to compute the AIF, is the fact that the measured MR signal saturates at moderately high [Gd]. This is due to the insensitivity of MR signal to changes in $\mathrm{T}_{2}^{*}$ as the measured $\mathrm{SI}$ will be below the noise floor $[48,49]$. Significant errors in the calculation of cerebral blood flow and cerebral blood volume have been observed when a SI-saturated AIF was used [48].

A solution to these issues is to use the MR phase to compute the AIF. The change in phase is dependent solely on the strength of the magnetic field $\left(\mathbf{B}_{\mathbf{0}}\right)$, the $T_{E}$ of the sequence, and the orientation of the vessel with respect to $\mathbf{B}_{\mathbf{0}}$. While $\mathbf{B}_{\mathbf{0}}$ and $T_{E}$ are known for each acquisition, the angle between the vessel and $\mathbf{B}_{\mathbf{0}}$ is easily calculable from acquired images. One of the main advantages in using $\phi$ is that the relationship between 
the change in $\phi$ and $[\mathrm{Gd}](\mathrm{t})$ is linear. This means that $\phi$ will not saturate over the entire range of $[\mathrm{Gd}]$ in the AIF. Using $\phi$ also has the added advantage of having a higher signalto-noise ratio (SNR) than an SI-based AIF [50]. Since the SI and $\phi$ data are acquired simultaneously within an MR acquisition, no additional sequences or calculations are required to use the $\phi$ data. It is for these reasons that $\phi$ data is the method of choice to acquire the AIF in this thesis.

\subsubsection{Arterial Input Function Versus Venous Output Function}

Quantitative DCE-MRI analysis relies heavily on the accurate measurement of the AIF as a bolus of GBCA enters a tissue of interest. There are many factors that can negatively affect the measurement of the AIF in a clinical DCE-MRI acquisition (e.g. PVA, inadequate temporal resolution, and patient movement). The latter two examples are difficult to moderate as the temporal resolution is limited by the need for higher spatial resolution while human subjects are prone to movement. The PVA, however, is a well-known effect in DCE-MRI and solutions have been investigated [51-54] yet no standard solution exists. A brief discussion of the reduction of PVA effects will be presented.

For the clinical study presented later in this thesis, brain tumours are the tissue of interest. This means that the AIF is typically measured within an artery in the head that directly feeds the brain tissue; some arteries that have been used previously are the internal carotid artery (ICA) and middle cerebral artery (MCA). The diameters of these arteries, for a healthy population, have been found to be approximately $5 \mathrm{~mm}$ [55] and 3 $\mathrm{mm}$ [56], respectively. The voxel size used in the clinical study presented later in the thesis 
was $1.7 \mathrm{~mm} \times 1.7 \mathrm{~mm} \times 5 \mathrm{~mm}$. In diagnostic imaging, it is not possible to ensure that any part of an imaged object is placed in a specific manner on a pixel grid. Therefore, with the dimensions of the pixel and MCA stated above, the chance that a single pixel would be wholly within the MCA is approximately only $40 \%$ while the chance that two pixels lie within the MCA is $0 \%$.

A demonstration of how the position of a vessel can affect the SI measurement on a $2 \times 2$ pixel grid is shown in Fig. 3.1. If the MCA is the vessel of choice and has a true $\mathrm{SI}$ of 50 and is surrounded by tissue with a true SI of 100, then for the placement of the vessel as shown in Fig. 3.1A all four pixels would have a measured $\mathrm{SI}$ of about 70. If the MCA was shifted, as in Fig. 3.1B, then the two left-most pixels have a measured SI of about 76 and the two right-most pixels have an SI of 65 . While the position of the $2 \times 2$ pixel grid does not change for each situation shown in Fig. 3.1, this example shows that, given the same composition of objects within a region of measurement, the SI can still vary on a pixel-by-pixel basis when the objects of interest are very small. This is a direct example of PVA.

To solve this issue, at least one of two things are required: either the pixel size is reduced, or the vessel needs to be larger. The spatial resolution of an MR acquisition is often a difficult option to improve as time considerations are a significant factor in clinical imaging. Therefore, the superior sagittal sinus (SSS) was chosen as the location to acquire $[\mathrm{Gd}](\mathrm{t})$ data.

It should be noted that the SSS is a vein and located in the top and back of the head. The diameter of the SSS, in healthy patients, is $9.9 \pm 2.4 \mathrm{~mm}$ [57]. This is nearly 

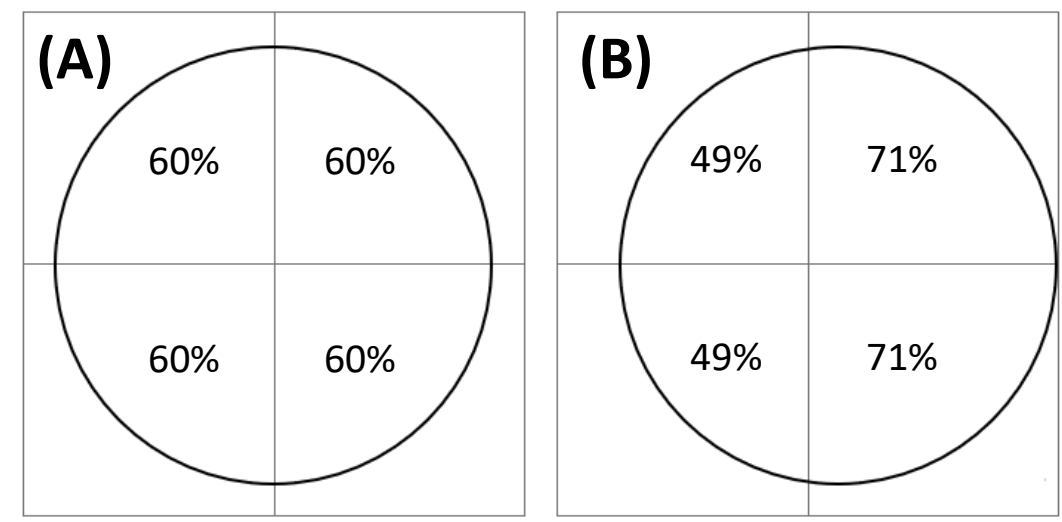

Figure 3.1: Representation of the location of the MCA on a pixel grid. (A) The MCA is in the center of four pixels and each pixel contains approximately $60 \% \mathrm{MCA}$, and $40 \%$ other tissues. (B) The MCA is placed in a shifted position, where the right-side pixels contain approximately $71 \%$ of MCA and the two left-side pixels are about $49 \%$.

twice as large as the ICA and three times as large as the MCA. This significantly larger size therefore allows for the placement of a region of interest (ROI) to include multiple pixels to lie wholly within the SSS. If the same pixel dimensions mentioned above are considered here, then a minimum of 16 pixels can lie within the SSS. This is a considerable factor in the reduction of PVA, as the ROI inside the SSS would only include blood and no other surrounding tissues. However, even though the ROI itself may not include surrounding tissues, their signal contributions may still contribute to the signal measured within the ROI by means of the point spread function.

One other consideration in using the VOF is the shape of the measured curve. In a clinical MR scan, GBCA is injected into a vein as a bolus where it passes through the heart, into the arterial system, and then finally back into the venous system. During that process, there will be a small amount of GBCA uptake by the body. Additionally, the GBCA bolus will undergo dispersion with time, such that the [Gd] peak of the VOF will be lower than 
the AIF peak. This also means that the [Gd] peak of the VOF will occur at a later point in time compared to the AIF. Regardless of these differences, the areas under the curve between these two acquisitions are nearly equal, which is important as this value represents the total volume of GBCA passing through the vascular system. With this, and the fact the VOF and AIF curves have very similar washout [Gd] values [58], indicates that their use in computing quantitative DCE-MRI parameters should, theoretically, produce similar results. In one study where the AIF was measured in multiple vessels, it was found that the 'AIF' measured in the SSS produced DCE-MRI parameters that were more accurate and more consistent than those measured in cranial arteries [59].

For these reasons, the simulations and clinical work presented in the rest of this thesis use the SSS as the vessel of measurement for all DCE-MRI acquisitions. The terminology AIF will still be used, however, to maintain consistency with the literature.

\subsection{Partial Volume Averaging}

For the DCE-MRI work in this thesis, AIF curves are presented for a phantom with flowing water, termed 'dynamic phantom', as well as for patients presenting with a high-grade glioma (HGG). In the following discussion, instead of referring specifically to an artery (or vein) or the tubing of the dynamic phantom, the more general term 'vessel' will be used to include both situations.

The AIF curves are generated from the average phase values measured as a function of time. These phase values are obtained by placing an ROI over the vessel of interest and calculating the mean phase over the ROI. This is done for several slices and 
the average over the slices is then computed to get the final value. In the following discussion, the average over slices will be ignored, and only the intra-slice situation will be considered. While the details may change from slice-to-slice, the general discussion should apply to all slices.

In the ideal case where the ROI and the pixels in the ROI line up exactly, such that none of the pixels span the border of the ROI, it is reasonable to assume that the average over the ROI is completely determined by the material inside the volume of tissue corresponding to the ROI. However, this is an ideal case, and it is much more common for pixels at the edge of the ROI to span this boundary. Consequently, an average over an ROI is implicitly contaminated by signal from outside the volume of interest.

In general, the placement of an ROI on an image will contain some pixels that are completely inside the object of interest, some that are completely outside, and some that have contributions from both regions. These three zones will be labelled 'in', 'out', and 'rim', respectively. For the rim-zone, a fraction, $f_{k}$, of the $k^{\text {th }}$ rim voxel volume (and pixel area) will be inside the vessel and $\left(1-f_{k}\right)$ of the voxel volume (and pixel area) will be outside the vessel. Thus, $f_{k}$ can be written as:

$$
f_{k}=\frac{V_{k}^{i n}}{V_{k}}=\frac{A_{k}^{i n}}{A_{k}},
$$

where $V_{k}$ is the total volume of the $k^{\text {th }}$ voxel and $V_{k}^{\text {in }}$ is the volume of voxel $k$ that is inside the vessel. $A_{k}$ and $A_{k}^{\text {in }}$ are the corresponding pixel areas. In general, $f_{k}$ will be different for each of the pixels in the rim-zone.

For each voxel in the rim-zone, the corresponding pixel value will be a weighted 
average of contributions from inside and outside of the vessel. Similarly, when an average over multiple pixels is computed, where at least one of the pixels is from the rim-zone, there will be contributions from both the interior and the exterior of the vessel. Both of these situations are examples of PVA.

MR images are generated from the measured MR signals by assigning a positive integer as the pixel value for each voxel such that the pixel value is proportional to the measured signal for the voxel and is constrained to be within a convenient range of values. Since the measured MR signal is complex, it will be useful to treat the resulting images and their pixel values as complex functions and numbers, respectively. In the following discussion, the $k^{\text {th }}$ pixel value, $P_{k}$, will be considered to be complex with $P_{R k}$ and $\mathrm{P}_{\mathrm{lk}}$ being the real and imaginary parts, respectively. Alternatively, $\mathrm{P}_{\mathrm{k}}$ can be expressed in terms of its magnitude and phase, denoted $\left|P_{k}\right|$ and $\phi_{k}$, respectively. $\left|P_{k}\right|$ and $\phi_{k}$ are computed from $\mathrm{P}_{\mathrm{Rk}}$ and $\mathrm{P}_{\mathrm{lk}}$ (which correspond to the complex signal that is actually measured for voxel k) using:

$$
\left|P_{k}\right|=\sqrt{P_{R k}^{2}+P_{l k}^{2}}
$$

and

$$
\phi_{\mathrm{k}}=\tan ^{-1}\left(\frac{\mathrm{P}_{\mathrm{lk}}}{\mathrm{P}_{\mathrm{Rk}}}\right) \text {, }
$$

respectively.

Pixel values from the interior, exterior, and rim-zones will be denoted $\mathrm{P}_{\mathrm{k}}^{\text {in }}, \mathrm{P}_{\mathrm{k}}^{\text {out }}$, and $\mathrm{P}_{\mathrm{k}}^{\mathrm{rim}}$, respectively. Using Eq. 3.1, $\mathrm{P}_{\mathrm{k}}^{\text {rim }}$ can be written in terms of $\mathrm{P}_{\mathrm{k}}^{\text {in }}$ and $\mathrm{P}_{\mathrm{k}}^{\text {out }}$ as:

$$
P_{k}^{\text {rim }}=f_{k} P_{k}^{\text {in }}+\left(1-f_{k}\right) P_{k}^{\text {out }}
$$




$$
P_{k}^{\text {rim }}=f_{k}\left(P_{R k}^{\text {in }}+i P_{l k}^{\text {in }}\right)+\left(1-f_{k}\right)\left(P_{R k}^{\text {out }}+i P_{l k}^{\text {out }}\right)
$$

For a complex image expressed in terms of its real and imaginary components, the mean complex pixel value averaged over the ROI can be written as:

$$
\begin{gathered}
\left\langle P_{k}\right\rangle=\left\langle P_{k}^{\text {in }}\right\rangle+\left\langle P_{k}^{\text {rim }}\right\rangle+\left\langle P_{k}^{\text {out }}\right\rangle \\
=\frac{1}{n} \sum_{k=1}^{n} P_{k}=\frac{1}{n} \sum_{k=1}^{n^{\text {in }}} P_{k}^{\text {in }}+\frac{1}{n} \sum_{k=1}^{n^{\text {rim }}} P_{k}^{\text {rim }}+\frac{1}{n} \sum_{k=1}^{n^{\text {out }}} P_{k}^{\text {out }} \\
=\frac{1}{n} \sum_{k=1}^{n^{\text {in }}} P_{k}^{\text {in }}+\frac{1}{n} \sum_{k=1}^{n^{\text {rim }}}\left[f_{k} P_{k}^{\text {in }}+\left(1-f_{k}\right) P_{k}^{\text {out }}\right]+\frac{1}{n} \sum_{k=1}^{n^{\text {out }}} P_{k}^{\text {out }} .
\end{gathered}
$$

For the case where all positions inside the vessel have the same MR signal (i.e. $P_{k}^{\text {in }}=P^{\text {in }}$ for all $k$ ) and all positions outside the vessel have the same MR signal but the extra-vessel signal is different from the intra-vessel signal (i.e. $P_{k}^{\text {out }}=\mathrm{P}^{\text {out }} \neq \mathrm{P}^{\text {in }}$ for all $k$ ), Eq. 3.7 simplifies to

$$
\begin{aligned}
& \left\langle P_{k}\right\rangle=\frac{1}{n}\left[n^{\text {in }} P^{\text {in }}+n^{\text {rim }} f_{k} P^{\text {in }}+n^{\text {rim }}\left(1-f_{k}\right) P^{\text {out }}+n^{\text {out }} p^{\text {out }}\right] \\
& =\frac{1}{n}\left\{\left[n^{\text {in }}+n^{\text {rim }} f_{k}\right] P^{\text {in }}+\left[n^{\text {rim }}\left(1-f_{k}\right)+n^{\text {out }}\right] P^{\text {out }}\right\} .
\end{aligned}
$$

This is just a weighted average of $\mathrm{P}^{\text {in }}$ and $\mathrm{P}^{\text {out }}$ where the same weighting applies to both the real and imaginary parts of complex pixel values. For the trivial case where $n^{\text {in }}=$ $\mathrm{n}^{\text {out }}=\mathrm{n}^{\text {rim }}=\mathrm{n} / 3$ and $\mathrm{f}_{\mathrm{k}}=0.5$, Eq. 3.8 reduces to

$$
\left\langle P_{k}\right\rangle=\frac{1}{2} P^{\text {in }}+\frac{1}{2} P^{\text {out }}
$$

as expected.

Although the MR signals are always measured in terms of their real and imaginary components, the corresponding real and imaginary images are not very useful. 
Magnitude images are used most of the time, but phase images are also important in certain situations (e.g. DCE-MRI). Unfortunately, the effects of PVA on these images do not reduce to a simple weighted average as was the case for $\mathrm{P}_{\mathrm{Rk}}$ and $\mathrm{P}_{\mathrm{Ik}}$.

For a magnitude image the value of pixel $k,\left|P_{k}\right|$, is calculated from $P_{R k}$ and $P_{l k}$ using Eq. 3.2. For interior, exterior, and rim-zone pixels, this gives:

$$
\begin{gathered}
\left|P_{k}^{\text {in }}\right|=\sqrt{\left(P_{R k}^{\text {in }}\right)^{2}+\left(P_{\mathrm{lk}}^{\text {in }}\right)^{2}} \\
\left|P_{k}^{\text {out }}\right|=\sqrt{\left(P_{R k}^{\text {out }}\right)^{2}+\left(P_{\mathrm{lk}}^{\text {out }}\right)^{2}} \\
\left|P_{k}^{\text {rim }}\right|=\sqrt{\left(P_{R k}^{\text {rim }}\right)^{2}+\left(P_{\mathrm{lk}}^{\text {rim }}\right)^{2}} \\
=\left\{\left[f_{k} P_{R k}^{\text {in }}+\left(1-f_{k}\right) P_{R k}^{\text {out }}\right]^{2}+\left[f_{k} P_{l k}^{\text {in }}+\left(1-f_{k}\right) P_{l k}^{\text {out }}\right]^{2}\right\}^{1 / 2} .
\end{gathered}
$$

Expressed in terms of the magnitude and phase of interior and exterior pixels, this becomes:

$$
\begin{aligned}
& \left|P_{k}^{\text {rim }}\right|=\left\{\left[f_{k}\left|P_{k}^{\text {in }}\right| \cos \phi_{k}^{\text {in }}+\left(1-f_{k}\right)\left|P_{k}^{\text {out }}\right| \cos \phi_{k}^{\text {out }}\right]^{2}\right. \\
& \left.+\left[f_{k}\left|P_{k}^{\text {in }}\right| \sin \phi_{k}^{\text {in }}+\left(1-f_{k}\right)\left|P_{k}^{\text {out }}\right| \sin \phi_{k}^{\text {out }}\right]^{2}\right\}^{1 / 2} .
\end{aligned}
$$

If $f_{k}^{\prime}$ is defined as:

$$
f_{k}^{\prime}=\frac{\left|P_{k}^{\text {out }}\right|}{\left|P_{k}^{\text {in }}\right|},
$$

then $\left|P_{k}^{\text {rim }}\right|$ can be written as

$$
\begin{aligned}
\left|P_{k}^{\text {rim }}\right|=\left|P_{k}^{\text {in }}\right|\left\{\left[f_{k} \cos \phi_{k}^{\text {in }}+\left(1-f_{k}\right) f_{k}^{\prime} \cos \phi_{k}^{\text {out }}\right]^{2}\right. & \\
& \left.+\left[f_{k} \sin \phi_{k}^{\text {in }}+\left(1-f_{k}\right) f_{k}^{\prime} \sin \phi_{k}^{\text {out }}\right]^{2}\right\}^{1 / 2}
\end{aligned}
$$


so that

$$
\begin{gathered}
\left|P_{k}^{\text {iim }}\right|=\left|P_{k}^{\text {in }}\right|\left\{f_{k}^{2} \cos ^{2} \phi_{k}^{\text {in }}+2 f_{k}\left(1-f_{k}\right) f_{k}^{\prime} \cos \phi_{k}^{\text {in }} \cos \phi_{k}^{\text {out }}+\left(1-f_{k}\right)^{2} f_{k}^{\prime 2} \cos ^{2} \phi_{k}^{\text {out }}\right. \\
\left.+f_{k}^{2} \sin ^{2} \phi_{k}^{\text {in }}+2 f_{k}\left(1-f_{k}\right) f_{k}^{\prime} \sin \phi_{k}^{\text {in }} \sin \phi_{k}^{\text {out }}+\left(1-f_{k}\right)^{2} f_{k}^{2} \sin ^{2} \phi_{k}^{\text {out }}\right\}^{1 / 2} \\
=\left|P_{k}^{\text {in }}\right|\left\{f_{k}^{2}+\left(1-f_{k}\right)^{2} f_{k}^{\prime 2}+2 f_{k}\left(1-f_{k}\right) f_{k}^{\prime} \cos \left(\phi_{k}^{\text {in }}-\phi_{k}^{\text {out }}\right)\right\}^{1 / 2}
\end{gathered}
$$

It can be seen from this equation that $\left|P_{k}^{\text {rim }}\right|$ is not a simple weighted average of $\left|P_{k}^{\text {in }}\right|$ and $\left|P_{\mathrm{k}}^{\text {out }}\right|$.

For a phase image, the value of pixel $k, \phi_{k}$, is calculated from $P_{R k}$ and $P_{I k}$ using Eq.

3.3. For interior, exterior, and rim-zone pixels, this gives:

$$
\begin{gathered}
\phi_{k}^{\text {in }}=\tan ^{-1}\left[\frac{P_{\mathrm{lk}}^{\text {in }}}{P_{\mathrm{Rk}}^{\text {in }}}\right] \\
\phi_{\mathrm{k}}^{\text {out }}=\tan ^{-1}\left[\frac{P_{\mathrm{lk}}^{\text {out }}}{P_{\mathrm{Rk}}^{\text {out }}}\right] \\
\phi_{\mathrm{k}}^{\text {rim }}=\tan ^{-1}\left[\frac{P_{\mathrm{lk}}^{\text {rim }}}{P_{\mathrm{Rk}}^{\text {rim }}}\right] \\
=\tan ^{-1}\left[\frac{f_{k} P_{\mathrm{lk}}^{\text {in }}+\left(1-f_{k}\right) P_{\mathrm{lk}}^{\text {out }}}{f_{\mathrm{k}} P_{\mathrm{Rk}}^{\text {in }}+\left(1-f_{k}\right) P_{\mathrm{Rk}}^{\text {out }}}\right] \\
=\tan ^{-1}\left[\frac{f_{k}\left|P_{k}^{\text {in }}\right| \sin \phi_{k}^{\text {in }}+\left(1-f_{k}\right)\left|P_{k}^{\text {out }}\right| \sin \phi_{k}^{\text {out }}}{f_{k}\left|P_{k}^{\text {in }}\right| \cos \phi_{k}^{\text {in }}+\left(1-f_{k}\right)\left|P_{k}^{\text {out }}\right| \cos \phi_{k}^{\text {out }}}\right] .
\end{gathered}
$$

Using Eq. 3.14, this can be written as:

$$
\phi_{k}^{\text {rim }}=\tan ^{-1}\left[\frac{f_{k} \sin \phi_{k}^{\text {in }}+\left(1-f_{k}\right) f_{k}^{\prime} \sin \phi_{k}^{\text {out }}}{f_{k} \cos \phi_{k}^{\text {in }}+\left(1-f_{k}\right) f_{k}^{\prime} \cos \phi_{k}^{\text {out }}}\right] .
$$

This can also be expressed as:

$$
\tan \phi_{k}^{\text {rim }}=\tan \left(\phi_{k}^{\text {in }}\right) \cdot\left[\frac{1+\left(1-f_{k}\right)\left(f_{k}^{\prime} / f_{k}\right)\left(\sin \phi_{k}^{\text {out }} / \sin \phi_{k}^{\text {in }}\right)}{1+\left(1-f_{k}\right)\left(f_{k}^{\prime} / f_{k}\right)\left(\cos \phi_{k}^{\text {out }} / \cos \phi_{k}^{\text {in }}\right)}\right]
$$


as long as $\sin \phi_{\mathrm{k}}^{\mathrm{in}} \neq 0, \cos \phi_{\mathrm{k}}^{\mathrm{in}} \neq 0$, and the denominator as a whole is not equal to zero. From Eq. 3.21, it can be seen that $\phi_{\mathrm{k}}^{\text {rim }}$ is not a simple weighted average of contributions from inside and outside the vessel.

The mean phase angle, averaged over the ROI, can be written as (see also Eq. 3.6):

$$
\left\langle\phi_{k}\right\rangle=\frac{1}{n} \sum_{k=1}^{n} \phi_{k}=\frac{1}{n} \sum_{k=1}^{n^{\text {in }}} \phi_{k}^{\text {in }}+\frac{1}{n} \sum_{k=1}^{n^{\text {rim }}} \phi_{k}^{\text {rim }}+\frac{1}{n} \sum_{k=1}^{n^{\text {out }}} \phi_{k}^{\text {out }}
$$

Again, it is difficult to simplify this expression in terms of the interior and exterior contributions. However, as with the magnitude, the trivial case where $\phi_{\mathrm{k}}^{\text {in }}=\phi_{\mathrm{k}}^{\text {out }}=\phi^{\text {in }}$ for all voxels yields the expected result of $\left\langle\phi_{k}\right\rangle=\phi^{\text {in }}$.

When the average phase over the ROI is assumed to be equal to the average phase of the signal coming from the vessel interior (i.e. $\left.\left\langle\phi_{\mathrm{k}}^{\mathrm{in}}\right\rangle\right)$ then $\left\langle\phi_{\mathrm{k}}^{\text {rim }}\right\rangle+\left\langle\phi_{\mathrm{k}}^{\text {out }}\right\rangle$ can be identified as an additive phase error, $\Delta \phi$ and Eq. 3.22 can be written as

$$
\left\langle\phi_{k}\right\rangle=\left\langle\phi_{k}^{\text {in }}\right\rangle+\Delta \phi
$$

where

$$
\begin{gathered}
\Delta \phi \equiv\left\langle\phi_{\mathrm{k}}^{\text {rim }}\right\rangle+\left\langle\phi_{\mathrm{k}}^{\text {out }}\right\rangle \\
=\frac{1}{\mathrm{n}} \sum_{\mathrm{k}=1}^{\mathrm{n}^{\text {rim }}} \phi_{\mathrm{k}}^{\text {rim }}+\frac{1}{\mathrm{n}} \sum_{\mathrm{k}=1}^{\mathrm{n}^{\text {out }}} \phi_{\mathrm{k}}^{\text {out }} .
\end{gathered}
$$

In principle, this phase error can be calculated using Eqs. 3.18 and 3.20; however, this is not very useful in practice, since the parameters $f_{k}, f_{k}^{\prime}, \phi_{k}^{\text {in }}$, and $\phi_{k}^{\text {out }}$ are not generally known. In fact, if the $\phi_{\mathrm{k}}^{\text {in }}$ were known, this analysis would not be necessary.

For the experimental work reported later in this thesis, the ROls used to obtain the average phase values were circular, they had nominally the same diameter as the 
vessel and they were centred with the vessel. In this case, there is no contribution from voxels that are completely outside the vessel. For these experiments, it is generally assumed that the GBCA is well mixed such that there is no phase variation over the interior of the vessel. This means that $\phi_{k}^{\text {in }}=\phi^{\text {in }}$ for all k. It also means the phase value in the interior part of all of the rim-zone voxels will be the same.

For this situation, Eq. 3.22 can be written as:

$$
\begin{gathered}
\left\langle\phi_{k}\right\rangle=\frac{n^{\text {in }}}{n} \phi^{\text {in }}+\frac{1}{n} \sum_{k=1}^{n^{\text {rim }}} \phi_{k}^{\text {rim }} \\
=\frac{n-n^{\text {rim }}}{n} \phi^{\text {in }}+\frac{1}{n} \sum_{k=1}^{n^{\text {rim }}} \phi_{k}^{\text {rim }} \\
=\phi^{\text {in }}+\frac{n^{\text {rim }}}{n}\left[\frac{1}{n^{\text {rim }}} \sum_{k=1}^{n^{\text {rim }}} \phi_{k}^{\text {rim }}-\phi^{\text {in }}\right] .
\end{gathered}
$$

If $\left\langle\phi_{k}\right\rangle$ and $\phi^{\text {in }}$ are identified as the measured and true values, respectively, the second term in Eq. 3.25 is an additive phase error and the equation can be written as

$$
\left\langle\phi_{\mathrm{k}}\right\rangle=\phi^{\mathrm{in}}+\Delta \phi_{\mathrm{PV}}
$$

where $\Delta \phi_{\mathrm{PV}}$, the phase error due to PVA, is:

$$
\Delta \phi_{P V}=\frac{n^{\text {rim }}}{n}\left[\frac{1}{n^{\text {rim }}} \sum_{k=1}^{n^{\text {rim }}} \phi_{k}^{\text {rim }}-\phi^{\text {in }}\right] .
$$

When a bolus of GBCA passes through the vessel, $f_{k}^{\prime}$ and $\phi^{\text {in }}$ will change with [Gd] which means that the phase error is different for each [Gd]. Since the magnitude of the signal inside the vessel increases with increasing [Gd], in the limit [Gd] $\rightarrow \infty, f_{k}^{\prime}=0$ for all $k$ and the phase error due to PVA disappears. The behaviour of this phase error term will 
be re-visited in Section 3.5, where examples using realistic parameter values will be discussed.

By inserting Eq. 3.20 into Eq. 3.27, the phase error can also be written as

$$
\Delta \phi_{P V}=\frac{n^{\text {rim }}}{n}\left\{\frac{1}{n^{\text {rim }}} \sum_{k=1}^{n^{\text {rim }}} \tan ^{-1}\left[\frac{f_{k} \sin \phi_{k}^{\text {in }}+\left(1-f_{k}\right) f_{k}^{\prime} \sin \phi_{k}^{\text {out }}}{f_{k} \cos \phi_{k}^{\text {in }}+\left(1-f_{k}\right) f_{k}^{\prime} \cos \phi_{k}^{\text {out }}}\right]-\phi^{\text {in }}\right\}
$$

It is often reasonable to assume that the material immediately outside the vessel is uniform enough that the signal in the external part of each rim-zone voxel can be considered to be the same. In other words, $f_{k}^{\prime}=f^{\prime}$ and $\phi_{k}^{\text {out }}=\phi^{\text {out }}$ for all rim-zone pixels. In this case, Eq. 3.28 simplifies to

$$
\Delta \phi_{P V}=\frac{n^{\text {rim }}}{n}\left\{\frac{1}{n^{\text {rim }}} \sum_{k=1}^{n^{\text {rim }}} \tan ^{-1}\left[\frac{f_{k} \sin \phi^{\text {in }}+\left(1-f_{k}\right) f^{\prime} \sin \phi^{\text {out }}}{f_{k} \cos \phi^{\text {in }}+\left(1-f_{k}\right) f^{\prime} \cos \phi^{\text {out }}}\right]-\phi^{\text {in }}\right\} .
$$

In most situations, the $f_{k}$ will be significantly different from one rim-zone pixel to another so it is not generally reasonable to assume that $f_{k}=f$ for all $k$. However, for the special case where this is true, the equation for $\Delta \phi_{\mathrm{PV}}$ further simplifies to

$$
\Delta \phi_{\mathrm{PV}}=\frac{\mathrm{n}^{\mathrm{rim}}}{\mathrm{n}}\left\{\tan ^{-1}\left[\frac{\mathrm{f} \sin \phi^{\text {in }}+(1-f) f^{\prime} \sin \phi^{\text {out }}}{f \cos \phi^{\text {in }}+(1-f) f^{\prime} \cos \phi^{\text {out }}}\right]-\phi^{\text {in }}\right\} .
$$

\subsection{MR Signal Phase During Slice Selection}

In the previous section, PVA was considered and an equation for the phase error caused by PVA was derived. However, it can easily be seen from Eq. 3.29 that the phase error vanishes when $\phi^{\text {in }}=\phi^{\text {out }}$. In this section, three sources of phase shift for the internal voxels relative to the external voxels will be examined: (i) a difference in magnetic 
susceptibility between the inside of the vessel and the outside, (ii) motion of the nuclear spins during the slice selection process, and (iii) the presence of a GBCA inside the vessel.

\subsubsection{Phase Change Due to Magnetic Susceptibility}

It was shown in Section 2.3.3 that the MR signal phase for a constant magnetic field, B, is given by Eq. 2.33. When the values of the magnetic susceptibility inside and outside of the vessel are not the same, the magnetic fields in these regions will differ and, as a result, the phases will also be different. The change in phase due to the different magnetic susceptibility values, $\Delta \phi_{\chi}$, is given by

$$
\Delta \phi_{\chi}=\phi^{\text {out }}-\phi^{\text {in }}=-\gamma\left(B^{\text {out }}-B^{\text {in }}\right) t
$$

where $B^{\text {out }}$ and $B^{\text {in }}$ are the magnetic fields outside (Eq. 2.91) and inside the vessel (Eq. 2.92), respectively [30].

When the vessel is modelled as a long straight cylinder where the length is much greater than the diameter the infinite cylinder approximation is often used. It has previously been shown that the infinite cylinder approximation is valid for measurements taken within the SSS [60]. Inserting the expressions for $\mathrm{B}^{\text {out }}$ and $\mathrm{B}^{\text {in }}$ into Eq. 3.31 and setting $t=T_{E}$ and $\omega_{0}=\gamma B_{0}$ gives:

$$
\begin{gathered}
\Delta \phi_{\chi}=-\left[\frac{\Delta \chi}{2} \omega_{0} \sin ^{2} \theta\left(\frac{r}{\xi}\right)^{2} \cos 2 \varphi+\frac{1}{3} \chi_{e} \omega_{0}-\frac{\Delta \chi}{2} \omega_{0}\left(\cos ^{2} \theta-\frac{1}{3}\right)-\frac{1}{3} \chi_{e} \omega_{0}\right] T_{E} \\
=-\frac{\Delta \chi}{2} \omega_{0} T_{E}\left[\sin ^{2} \theta\left(\frac{r}{\xi}\right)^{2} \cos 2 \varphi-\cos ^{2} \theta+\frac{1}{3}\right]
\end{gathered}
$$

where $r$ is the radius of the cylinder, $\theta$ is the angle between $B_{0}$ and the $z$-axis, $\xi$ is the 
distance from the central axis to the point of measurement, $\varphi$ is the azimuthal angle to $\xi$, $\Delta x=\chi_{i}-\chi_{e^{\prime}} \chi_{i}$ is the magnetic susceptibility inside the cylinder, and $\chi_{e}$ is the magnetic susceptibility of surrounding tissue.

This equation is complicated by the presence of $r$ and $\varphi$ which suggests that $\Delta \phi$ will depend on the voxel position outside the vessel. To simplify matters, $\Delta \phi_{\chi}$ averaged over all $\varphi$ could be considered. In this case, Eq. 3.32 reduces to

$$
\left\langle\phi_{\chi}\right\rangle=\frac{1}{2} \Delta \chi \omega_{0} T_{E}\left(\cos ^{2} \theta-\frac{1}{3}\right)
$$

This is consistent with the equations given in the literature for $\Delta \phi_{\chi}$ [61]. However, it is not necessary to introduce this average for the situation considered here since the only case of interest is when the cylinder is parallel to the applied magnetic field, $\mathbf{B}_{\mathbf{0}}$. Accordingly, when $\theta=0$ is inserted into Eq. 3.32 it simplifies to

$$
\Delta \phi_{\chi}=\frac{1}{3} \Delta \chi \omega_{0} T_{E}
$$

According to Haacke et al. [30], the magnetic susceptibility of the total blood system, $\chi_{b}$, can be expressed in terms of the blood oxygen concentration, $Y$, and the hematocrit, Hct, as:

$$
\chi_{b}=H c t\left[Y \chi_{o x}+(1-Y) \chi_{d}\right]+(1-H c t) X_{p}
$$

where $\chi_{o x}, \chi_{d}$ and $\chi_{p}$ are the magnetic susceptibilities for fully oxygenated blood, fully deoxygenated blood, and plasma, respectively.

It has also been observed that $\chi_{\mathrm{ox}} \approx \chi_{\mathrm{e}}$ and it is expected that $\chi_{\mathrm{p}} \approx \chi_{\mathrm{ox}} \approx \chi_{\mathrm{e}}[30]$. Based on this and Eq. 3.35, the magnetic susceptibility difference between inside and outside of the vessel, $\Delta \mathrm{\chi}$, can be written 


$$
\begin{aligned}
& \Delta \chi=\chi_{i}-\chi_{e}=\chi_{b}-\chi_{e} \\
= & \operatorname{HctY} \chi_{o x}+\operatorname{Hct} \chi_{d}-\operatorname{HctY} \chi_{d}+\chi_{o x}-H c t \chi_{o x}-\chi_{o x} \\
= & \operatorname{Hct}_{\mathrm{ox}}(Y-1)+\operatorname{Hct}_{\mathrm{d}}(1-Y) \\
= & \operatorname{Hct}_{\mathrm{do}}(1-Y)
\end{aligned}
$$

where

$$
\chi_{\mathrm{do}} \equiv \chi_{\mathrm{d}}-\chi_{\mathrm{ox}}
$$

The value of $\chi_{\text {do }}$ has been measured by several groups. Weisskoff et al. [62] reported a value of $0.18 \mathrm{ppm}$ (in cgs units) whereas more recently Spees et al. [63] and Jain et al. [64] both reported $\chi_{\text {do }}=0.27 \mathrm{ppm}$ (in cgs units). The more recent work will be considered to be the most reliable. Accordingly, $\chi_{\mathrm{do}}=4 \pi \cdot 2.7 \times 10^{-7}$ (in SI units) will be used here.

Inserting this back into Eq. 3.36 and then Eq. 3.34 gives:

$$
\Delta \phi_{\chi}=\phi^{\text {out }}-\phi^{\text {in }}=\frac{1}{3} H_{c t} \chi_{\text {do }}(1-Y) \omega_{0} T_{E}
$$

If it is assumed that (i) the reference frequency and phase are the same for the transmitter, receiver, and rotating frame, (ii) the frequency is set to the resonance frequency of the extra-vessel material, and (iii) the initial phase of the RF pulse is zero, then for an RF pulse applied along the $\hat{x}^{\prime}$-axis, $\phi^{\text {out }}$ will be equal to $\pi / 2$ and $\phi^{\text {in }}$ will be given by

$$
\phi^{\text {in }}=\phi^{\text {out }}-\Delta \phi_{\chi}=\frac{\pi}{2}-\frac{1}{3} \operatorname{Hct}_{\mathrm{do}}(1-Y) \omega_{0} T_{E}
$$

The value of Hct, on average, is approximately 0.40 for women and 0.45 for men $[18,30]$. The value of $Y$ can be expected to change from person to person and with activity level. Haacke et al. [30] report a resting state value of $Y=0.544$. 


\subsubsection{Phase Change Due to Blood Flow}

In Chapter 2, it was shown that when an RF pulse with phase $\phi_{\mathrm{RF}}$ is applied to a system of on-resonance stationary spins the magnetization is tipped from an orientation parallel to $\hat{\mathbf{z}}^{\prime}$ towards the $x^{\prime}-y^{\prime}$ plane where the $x^{\prime} y^{\prime}$ component will have a phase of $\phi_{R F}+\pi / 2$. The situation, however, is more complicated in the presence of a magnetic field gradient when the spins are moving along that gradient. In this section, the phase after the slice selection process will be considered for spins moving perpendicular to an axial slice. In the following discussion it will be assumed that $\phi_{\mathrm{RF}}=0$, for convenience.

In Section 2.4.1, it was shown that the phase after slice selection for an axial slice is $\pi / 2$ for stationary spins (see Eq. 2.55 ). It was also shown that the phase at time t during the slice selection process is given by Eq. 2.54 (see also ref. 27):

$$
\phi(z(t), t)=-\gamma \int_{0}^{t} G_{z}\left(t^{\prime}\right)\left[z\left(t^{\prime}\right)-z_{c}\right] d t^{\prime}+\gamma G_{z}\left[z(t)-z_{c}\right] \frac{T_{R F}}{2}+\frac{\pi}{2},
$$

where $G_{z}$ is the amplitude of the slice selection gradient and $T_{R F}$ is the duration of the RFpulse. In this equation, explicit time dependence for $z$ was inserted. The phase after the slice selection process corresponds to $t \geq \frac{3}{2} T_{R F}$ where

$$
G_{z}(t)=\left\{\begin{array}{ccc}
G_{z} & \text { for } & t=0 \text { to } T_{R F} \\
-G_{z} & \text { for } & t=T_{R F} \text { to } 3 / 2 T_{R F} \\
0 & \text { for } & t>3 / 2 T_{R F}
\end{array}\right.
$$

When there is no blood flow to consider, the first two terms in this equation cancel and the phase after the slice selection process, $\phi\left(z, \frac{3}{2} T_{R F}\right)$, is $\pi / 2$.

When blood flow in the direction of the slice selection gradient (i.e. the z-direction 
for an axial acquisition) is present, the first two terms in Eq. 3.40 will not cancel causing the phase angle to differ from $\pi / 2$ by $\Delta \phi_{F}$, Thus for this case

$$
\phi\left(\mathrm{z}(\mathrm{t}), \frac{3}{2} \mathrm{~T}_{\mathrm{RF}}\right)=\frac{\pi}{2}+\Delta \phi_{\mathrm{F}}
$$

Blood flow can be introduced into the calculation by assuming that spins are at position $z_{1}$ for the full duration of the slice selection gradient and they jump instantaneously to position $z_{2}$ for the full duration of the refocussing gradient. For this model, the phase can be calculated from Eq. 3.40 to give:

$$
\begin{gathered}
\phi\left(z(t), t \geq \frac{3}{2} T_{R F}\right)=\phi\left(z(t), t=\frac{3}{2} T_{R F}\right) \\
=-\nu \int_{0}^{3 / 2^{T} T_{R F}} G_{z}\left(t^{\prime}\right)\left[z\left(t^{\prime}\right)-z_{c}\right] d t^{\prime}+\gamma G_{z}\left[z(t)-z_{c}\right] \frac{T_{R F}}{2}+\frac{\pi}{2} \\
=-\nu G_{z}\left(z_{1}-z_{c}\right) T_{R F}-\gamma\left(-G_{z}\right)\left(z_{2}-z_{c}\right)\left(\frac{3}{2} T_{R F}-T_{R F}\right)+\gamma G_{z}\left(z_{1}-z_{c}\right) \frac{T_{R F}}{2}+\frac{\pi}{2} \\
=-\gamma G_{z}\left[\left(z_{1}-z_{c}\right) T_{R F}-\left(z_{2}-z_{c}\right) \frac{T_{R F}}{2}-\left(z_{1}-z_{c}\right) \frac{T_{R F}}{2}\right]+\frac{\pi}{2} \\
=-\nu G_{z} \frac{T_{R F}}{2}\left(z_{1}-z_{2}\right)+\frac{\pi}{2} .
\end{gathered}
$$

From Eqs. 3.42 and 3.43, it can be seen that the change in phase due to the blood flow, $\Delta \phi_{F}$, is given by

$$
\Delta \phi_{\mathrm{F}}=-\gamma \mathrm{G}_{\mathrm{z}} \frac{\mathrm{T}_{\mathrm{RF}}}{2}\left(\mathrm{z}_{1}-\mathrm{z}_{2}\right) .
$$

Note that this expression is independent of position within the slice; it just depends on the displacement. For stationary spins, $\Delta \phi_{\mathrm{F}}$ will be zero, as expected. When the spins are moving in the head-to-foot direction, as would be the case for the venous blood in the superior sagittal sinus, $z_{1}>z_{2}$, which means that the phase will be less than $\pi / 2$. For 
motion in the opposite direction, the phase will be greater than $\pi / 2$.

Since the duration of the refocussing gradient is half the duration of the slice selection gradient, the phase acquisition during the RF-pulse can be considered to occur from $t=\frac{1}{2} T_{R F}$ to $T_{R F}$. For constant fluid flow in the vessel, $z_{1}$ can be considered to be the average position over this time interval while $z_{2}$ can be considered to the average position over the time interval $t=T_{R F}$ to $\frac{3}{2} T_{R F}$. Thus, $z_{2}-z_{1}$ corresponds to the displacement that occurs over a time period of $\Delta t=\frac{5}{4} \mathrm{~T}_{\mathrm{RF}}-\frac{3}{4} \mathrm{~T}_{\mathrm{RF}}=\frac{1}{2} \mathrm{~T}_{\mathrm{RF}}$. Consider a vessel of diameter $\mathrm{d}$, with a constant flow, $F$, given by

$$
F=\pi\left(\frac{d}{2}\right)^{2} \frac{\Delta z}{\Delta t}
$$

If $\mathrm{F}$ and $\mathrm{d}$ are known, the displacement $\Delta \mathrm{z}=\mathrm{z}_{2}-\mathrm{z}_{1}$ of the fluid during the slice selection process can be expressed in terms of these physiological parameters and the sequence parameter $\mathrm{T}_{\mathrm{RF}}$ as

$$
\Delta z=\frac{4 T_{R F} F}{\pi d^{2}} .
$$

Inserting this back into Eq. 3.42 gives:

$$
\begin{gathered}
\phi\left(\mathrm{z}(\mathrm{t}), \mathrm{t}=\frac{3}{2} \mathrm{~T}_{\mathrm{RF}}\right)=\frac{\pi}{2}+\Delta \phi_{\mathrm{F}} \\
=\frac{\pi}{2}-\frac{\gamma \mathrm{G}_{\mathrm{z}} \mathrm{T}_{\mathrm{RF}}}{2} \frac{4 \mathrm{~T}_{\mathrm{RF}} \mathrm{F}}{\pi \mathrm{d}^{2}} \\
=\frac{\pi}{2}-\frac{2 \gamma \mathrm{G}_{\mathrm{z}} \mathrm{T}_{\mathrm{RF}}^{2} \mathrm{~F}}{\pi \mathrm{d}^{2}}
\end{gathered}
$$

and thus

$$
\Delta \phi_{\mathrm{F}}=-\frac{2 \gamma \mathrm{G}_{\mathrm{z}} \mathrm{T}_{\mathrm{RF}}^{2} \mathrm{~F}}{\pi \mathrm{d}^{2}} .
$$


For blood moving in the SSS, the flow will be negative since $\Delta z<0$. For convenience, flow will be treated here as a positive quantity and a negative sign was explicitly inserted into Eq. 3.47 as a result. Consequently, Eq. 3.47 and 3.48 are only strictly valid for blood flow in the head to foot direction, the only situation of interest for the work presented in this thesis.

\subsubsection{Phase Change Due to a GBCA}

When a GBCA is present inside a vessel but not outside, $\phi^{\text {in }}$ will be different from $\phi^{\text {out }}$. Furthermore, $\phi^{\text {in }}$ will depend on [Gd]. This is the principle on which the use of phase for measuring the AIF is based. However, it can be seen from Eq. 3.30 that the phase error (i.e. error due to PVA) is also a function of $\phi^{\text {in }}$. Thus, the value of $\phi^{\text {in }}$ used to calculate the phase error will be affected by [Gd].

The change in phase from the baseline phase caused by the presence of a GBCA is given by Eqs. 2.109 and 2.110 as [47]:

$$
\Delta \phi_{\mathrm{GBCA}}=-\frac{\omega_{0} T_{\mathrm{E}}}{2} \chi_{\mathrm{m}}[\mathrm{Gd}]\left(\cos ^{2} \theta-\frac{1}{3}\right)
$$

where the notation $\Delta \phi_{\mathrm{GBCA}}$ has been introduced to be consistent with Sections 3.4.1 and 3.4.2.

\subsubsection{Intra-vessel Phase}

From the discussion presented above, the phase at time $T_{E}$ for blood flowing inside the vessel where $[\mathrm{Gd}]>0$ is given by 


$$
\phi^{\text {in }}=\frac{\pi}{2}-\Delta \phi_{\mathrm{GBCA}}+\Delta \phi_{\chi}+\Delta \phi_{\mathrm{F}}
$$

where $\Delta \phi_{\mathrm{GBCA}}$ is the change in phase due to the presence of the GBCA, $\Delta \phi_{\chi}$ is the change in phase due to the change in magnetic susceptibility, and $\Delta \phi_{F}$ is the change in phase due to blood flow. If the average over all interior and rim-zone voxels is computed, per Eq. 3.26:

$$
\begin{gathered}
\left\langle\phi_{\mathrm{k}}\right\rangle=\phi^{\mathrm{in}}+\Delta \phi_{\mathrm{PV}} \\
=\frac{\pi}{2}-\Delta \phi_{\mathrm{GBCA}}+\Delta \phi_{\chi}+\Delta \phi_{\mathrm{F}}+\Delta \phi_{\mathrm{PV}} .
\end{gathered}
$$

When $[\mathrm{Gd}]=0$, Eq. 3.51 gives the baseline phase as measured with an ROI as

$$
\left\langle\phi_{\mathrm{k}}\right\rangle_{0}=\frac{\pi}{2}+\Delta \phi_{\chi}+\Delta \phi_{\mathrm{F}}+\Delta \phi_{\mathrm{PV}, 0}
$$

where $\Delta \phi_{\mathrm{PV}, 0}$ is the value of $\Delta \phi_{\mathrm{PV}}$ when [Gd] $=0$. From Eqs. 3.38 and 3.48, it is apparent that $\Delta \phi_{\chi}$ and $\Delta \phi_{F}$ are not affected by the presence of a GBCA, respectively. However, the same cannot be said for $\Delta \phi_{\mathrm{PV}}$. Thus, for phases averaged over the ROI, the difference between the phase values for $[\mathrm{Gd}]>0$ and $[\mathrm{Gd}]=0$ is given by

$$
\left\langle\phi_{\mathrm{k}}\right\rangle-\left\langle\phi_{\mathrm{k}}\right\rangle_{0}=-\frac{\omega_{0} \mathrm{~T}_{\mathrm{E}}}{2} \chi_{\mathrm{m}}[\mathrm{Gd}]\left(\cos ^{2} \theta-\frac{1}{3}\right)+\Delta \phi_{\mathrm{PV}}-\Delta \phi_{\mathrm{PV}, 0}
$$

where the term $\Delta \phi_{\mathrm{GBCA}}$ has been replaced with Eq. 3.49. This is representative of the actual experimental situation. Note that $\Delta \phi_{\chi}$ and $\Delta \phi_{F}$ do not appear explicitly in Eq. 3.53. However, since they both affect $\phi^{\text {in }}$ (see Eq. 3.50), it can be seen from Eq. 3.30 that they will have an effect on $\Delta \phi_{\mathrm{PV}}$ and, therefore, cannot be ignored.

While the difference considered in Eq. 3.53 is between the phase for $[\mathrm{Gd}]>0$ and $[G d]=0$, any two $[G d]$ values could have been considered. For example, if the first term 
is the GBCA concentration at time $\tau$ and the second term is the concentration corresponding to the AIF washout (i.e. at time $\tau_{w}$ ), the expression becomes

$$
\begin{aligned}
\left\langle\phi_{\mathrm{k}}\right\rangle(\tau)-\left\langle\phi_{\mathrm{k}}\right\rangle\left(\tau_{\mathrm{w}}\right)= & -\frac{\omega_{0} \mathrm{~T}_{\mathrm{E}}}{2} \chi_{\mathrm{m}}\left\{[\mathrm{Gd}](\tau)-[\mathrm{Gd}]\left(\tau_{\mathrm{w}}\right)\right\}\left(\cos ^{2} \theta-\frac{1}{3}\right) \\
& +\Delta \phi_{\mathrm{PV}}(\tau)-\Delta \phi_{\mathrm{PV}}\left(\tau_{\mathrm{w}}\right) .
\end{aligned}
$$

Again, $\Delta \phi_{\chi}$ and $\Delta \phi_{\mathrm{F}}$ cancel out.

\subsection{Phase Error Computations For Realistic Parameters}

In Section 3.3, a general expression was derived for the MR signal phase averaged over an ROI with PVA considered explicitly (see Eq. 3.22). When the ROI is the same size and shape as the vessel, it is centred with the vessel and the phase variation across the interior of the vessel is negligible, it was shown in Eq. 3.26 that the average phase is the sum of the true phase and a phase error term due to PVA, $\Delta \phi_{\mathrm{PV}}$. When the material immediately outside the vessel is uniform enough that it is reasonable to assume that $f_{k}^{\prime}=f^{\prime}$ and $\phi_{\mathrm{k}}^{\text {out }}=\phi^{\text {out }}$ for all $\mathrm{k}$, then $\Delta \phi_{\mathrm{PV}}$ is given by Eq. 3.29.

In Section 3.4, it was noted that $\Delta \phi_{\mathrm{PV}}=0$ when $\phi^{\text {in }}=\phi^{\text {out}}$; however, it was suggested that this is not likely to be the case for the vessels considered in this thesis. Three sources of phase shift between the interior and exterior of the vessel were presented: (i) different magnetic susceptibilities for inside and outside of the vessel; (ii) intra-vessel flow during the slice selection process; and (iii) the presence of a GBCA inside the vessel. In this section, each of these will be considered separately to determine their effects on the $\Delta \phi_{\mathrm{PV}}$ values. Anticipating the experimental work presented later in the 
thesis, values of $\Delta \phi_{\mathrm{PV}}$ will be computed using Eq. 3.29 with vessel, sequence, and ROI parameters relevant to this work.

\subsubsection{Vessel Properties}

For both the SSS and the dynamic phantom tubing, the vessel is considered to be round with an inside diameter, $d$, of $10 \mathrm{~mm}$. While the flow in the SSS is about $280 \mathrm{~cm}^{3} \mathrm{~min}^{-1}$ [65], an $\mathrm{F}$ value of $220 \mathrm{~cm}^{3} \mathrm{~min}^{-1}$, or $3.7 \mathrm{~cm}^{3} \mathrm{~s}^{-1}$, was used to approximate the flow rate set in the phantom experiments performed in Chapter 5 (flow was set between 140 and $\left.250 \mathrm{~cm}^{3} \min ^{-1}\right)$.

It should be noted that for the arterial system, the blood flow is very pulsatile and the normal blood velocity profile is generally laminar at systole, which means that the flow varies significantly both in time and in position within the vessel lumen [66]. Venous blood flow, however, is not pulsatile and the velocity profile is quite uniform. Thus, it is reasonable to assume that $F$ is constant in the SSS whereas if an artery was being considered, this assumption would not be valid.

\subsubsection{Sequence Properties}

The MRI sequence used for the AIF measurements reported later in the thesis is a dual echo 3D FLASH sequence. The details of the RF and gradient pulses are not available; however, it should be possible to make reasonable estimates. For the purposes of the calculations done in this section, $T_{R F}$ will be considered to be between 1 and 2 ms and $G_{z}$ 
will be assumed to be between 10 and $20 \mathrm{mT} \mathrm{m}^{-1}$.

\subsubsection{ROI and Vessel Models}

\subsubsection{Circular Vessel and ROI}

Consider the following situation for the vessel and ROI model:

a) the vessel of interest has a circular cross section and inner dimeter of $10 \mathrm{~mm}$;

b) the vessel has a wall thickness of $0.5 \mathrm{~mm}$ and the wall contributes no signal;

c) the image pixels are $2 \mathrm{~mm} \times 2 \mathrm{~mm}$;

d) the ROI is circular, has a diameter of $5 \mathrm{~mm}$ and is centered with the vessel.

This vessel and ROI model system is shown in Fig. 3.2 for three different situations: (i) the vessel is centred with the pixel grid; (ii) the vessel is shifted by a half pixel in one direction; and (iii) the vessel is shifted by a half pixel in both directions. Note, when the rim-zone pixels are considered, the circular ROI effectively becomes a square in Fig. 3.2A and an irregular shape in Figs 3.2B and 3.2C.

The variables $n, n^{\text {in }}$, and $n^{\text {out }}$ were determined by inspection for the three cases shown in Fig 3.2 and the values are given in Table 3.1. From this analysis, $n^{r i m} / n$ can be expected to be approximately $40-50 \%$ for the model system being considered. It can also be seen from Fig. 3.2 that the inside of the vessel corresponds to approximately $70-$ $85 \%$ of the total pixel area.

It is also apparent from Fig. 3.2 that the $f_{k}$ will be different, in general, for each pixel in the rim-zone. This is caused primarily by having a circular vessel and a circular ROI on a square pixel grid. Another factor that will affect $f_{k}$ is any offset of the ROI from the 


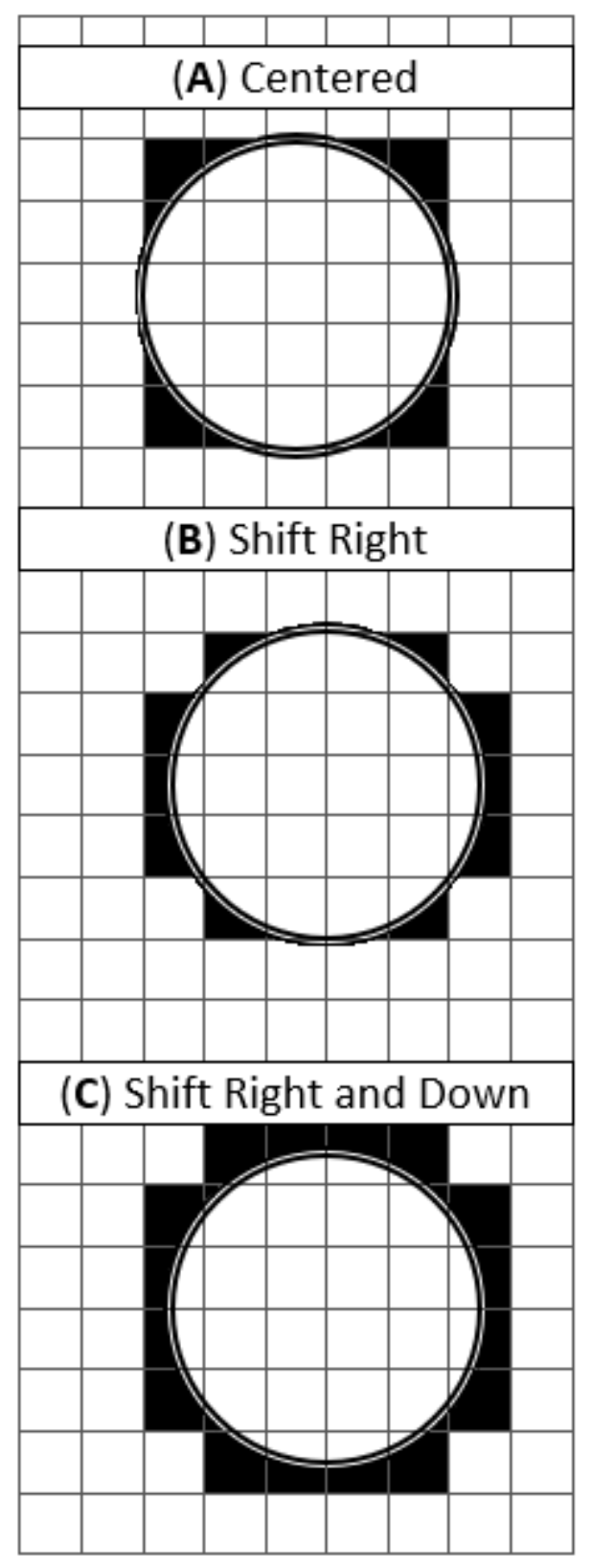

Figure 3.2: Circular vessel and ROI model used in phase error calculations: $(\mathbf{A})$ the vessel is centered with respect to the pixel grid; (B) the vessel is shifted by half of a pixel to the right; (C) the vessel is shifted to the right by half of a pixel and down by half a pixel. 
Table 3.1: Pixel fractions for the three circular vessels shown in Fig. 3.2.

\begin{tabular}{cccccc}
\hline Case & $\mathrm{n}$ & $\mathrm{n}^{\text {in }}$ & $\mathrm{n}^{\text {in }} / \mathrm{n}(\%)$ & $\mathrm{n}^{\text {rim }}$ & $\mathrm{n}^{\text {rim }} / \mathrm{n}(\%)$ \\
\hline 1 & 25 & 13 & 52.0 & 12 & 48.0 \\
2 & 26 & 16 & 61.5 & 10 & 38.5 \\
3 & 32 & 16 & 50.0 & 16 & 50.0 \\
\hline
\end{tabular}

vessel. The figure shows only the case where they are perfectly aligned. The examples shown in Fig. 3.2 also illustrate that $f_{k}$ may have values approaching 0 and $100 \%$ on the same image. By inspection, it can also be seen from Fig. 3.2 that, for these examples, the total fraction of the rim-zone voxel volume (not including the vessel wall) that corresponds to the inside of the vessel is roughly $75 \%, 50 \%$, and $25 \%$ for Fig. $3.2 \mathrm{~A}$ to $3.2 \mathrm{C}$, respectively.

\subsubsection{Square Vessel and ROI}

The ROI shape does not directly influence the equation for $\Delta \phi_{\mathrm{PV}}$ given in Eq. 3.29. The ROI shape does affect the values of $n, n^{\text {rim }}$, and the $f_{k}$; however, any ROI that has similar values for these parameters will give similar results for $\Delta \phi_{\mathrm{PV}}$. In a similar way, it can be argued that the shape of the vessel considered does not affect the $\Delta \phi_{\mathrm{PV}}$ values computed with Eq. 3.29. With this rationale, a square model is introduced.

Consider the following (see Fig. 3.3):

a) the vessel of interest has a square cross-section where the side length is between 10 and $14 \mathrm{~mm}$; 
b) the vessel wall thickness is negligible, or equivalently, it behaves the same as the extra-vessel space;

c) the image pixels are $2 \mathrm{~mm} \times 2 \mathrm{~mm}$;

d) the ROI has an irregular "plus-sign-like" shape;

e) the ROI is centred with the vessel;

f) the vessel is offset from the pixel grid in both directions.

For this model $n=45, n^{\text {in }}=25(55.6 \%)$, and $n^{\text {rim }}=20(44.4 \%)$.

The main advantage of using this square vessel model is that the $f_{k}$ will be the same for all rim-zone pixels. This simplifies the computation of $\Delta \phi_{\mathrm{PV}}$ considerably. In the example shown in Fig. 3.3, $f_{k}=0.5$ for all rim-zone pixels. Other values of $f_{k}$, where $f_{k}=f$ for all rim-zone pixels, can be considered by changing the size of the square vessel for the same ROI. As the vessel wall length decreases from $12 \mathrm{~mm}$ (the size shown in Fig. 3.3) to $10 \mathrm{~mm}$, the fraction of the rim-zone pixels that corresponds to the inside of the vessel reduces to zero (i.e. $f_{k}=f \rightarrow 0$ ) for all rim-zone pixels. Conversely, as the vessel wall length increases to $14 \mathrm{~mm}, f_{k}=f \rightarrow 1$ for all rim-zone pixels. It should be noted that since neither $\left\langle\phi_{k}\right\rangle$ nor $\phi^{\text {rim }}$ depend on vessel size explicitly (see Eqs. 3.25 and 3.20, respectively), changing the vessel size does not introduce any loss of generality.

Since $f$ can have any value between 0 and 1 with approximately equal likelihood, $\mathrm{f}$ will be treated as an independent variable for the purposes of these computations. For the two special cases of $f=1.0$ and $f=0.0$, the phase errors can easily be determined from Eq. 3.29 to be $\Delta \phi_{P V}=0$ and $\Delta \phi_{P V}=\frac{n^{\text {rim }}}{n}\left(\phi^{\text {out }}-\phi^{\text {in }}\right)$. However, since $f=0.0$ and $f=$ 1.0 cannot occur with a circular vessel on a square pixel grid, these cases will not be 


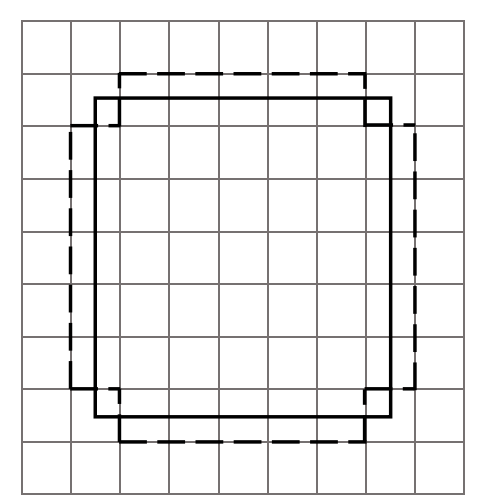

Figure 3.3: The square pixel method used in the phase error simulations. Here, the solid square represents the vessel and the dashed plus-sign line represents the chosen ROI.

considered further. $f$ values considered will be restricted to the range $0.25 \leq f \leq 0.75$.

The values of $f^{\prime}$ can, in principle, be determined experimentally from the magnitude images that are acquired at the same time as phase images. When $[\mathrm{Gd}]=0$, the vessel is normally darker than the surrounding tissue. Based on this empirical observation, $f^{\prime}$ is considered here to be approximately equal to 1.2. As [Gd] increases, the vessel becomes much brighter and $f^{\prime}$ decreases accordingly. For the peak of the AIF, the signal magnitude inside the vessel will be considered to be about 15 times larger than the magnitude of the signal coming from outside the vessel. For the washout section of the AIF, it will be considered to be about 5 times greater. This gives $f^{\prime}$ values of 0.20 and 0.067 for the AIF washout and peak, respectively. The corresponding [Gd] values for the baseline, washout, and peak will be considered to be $0,0.5$, and $6 \mathrm{mM}$, respectively.

Three sources of phase shift between the interior and exterior of the vessel were previously introduced in Section 3.4. All three sources affect $\phi^{\text {in }}$, the phase inside the vessel, as well as the $\left\langle\phi_{k}\right\rangle$, the ROI averaged phase. This was shown in Eqs. 3.50 and 3.51. 
However, when the phase difference for $[G d]>0$ relative to baseline (i.e. $[G d]=0$ ) is considered, $\Delta \phi_{\chi}$ and $\Delta \phi_{F}$ cancel out and do not appear explicitly in the resulting equation (see Eq. 3.53). While $\Delta \phi_{\chi}$ and $\Delta \phi_{F}$ are not explicitly in the equation for the phase difference relative to baseline, they do affect $\Delta \phi_{\mathrm{PV}}$ via their effect on $\phi^{\text {in }}$ (see Eqs. 3.29 and 3.50) and therefore cannot be ignored.

In Section 2.4.1, it was shown that the phase after slice selection is $\pi / 2$ for stationary spins when $\phi_{\mathrm{RF}}=0,[\mathrm{Gd}]=0$, and the magnetic susceptibility of the location being considered is the same as for the space exterior to the vessel. All of these conditions apply to the extra-vessel space so that

$$
\phi^{\text {out }}=\frac{\pi}{2}
$$

For the intra-vessel space, the situation is more complicated. The nominal value for $\phi^{\text {in }}$ will be considered to be $\pi / 2$ and the effects of $\chi^{\text {in }} \neq \chi^{\text {out }}, F \neq 0$, and $[G d] \neq 0$ will be introduced as separate phase shifts. In Section 3.4, $\phi^{\text {in }}$ was given in Eq. 3.50 where $\Delta \phi_{\chi^{\prime}}, \Delta \phi_{\mathrm{F}}$, and $\Delta \phi_{\mathrm{GBCA}}$ were defined in Eqs. 3.38, 3.48, and 3.49, respectively.

Values of $\Delta \phi_{\mathrm{PV}}$ can now be calculated using the parameter values discussed above and summarized in Table 3.2. Note that the Hct values were given for females, males, and an average value of 0.431 which reflects the subject group for the clinical study reported later in the thesis. For this study, there were 13 males and 8 females. $\Delta \phi_{\mathrm{PV}}$ values were calculated for the baseline, the washout, and the peak. The $f^{\prime}$ values used for these three scenarios were $1.2,0.2$, and 0.067 , respectively. The corresponding [Gd] values were considered to be $0,0.5$, and $6 \mathrm{mM}$, respectively. 
Table 3.2: Summary of parameters used in $\Delta \phi_{\mathrm{PV}}$ calculations. The Hct value of 0.40 was used for females, 0.45 for males, and 0.431 as a weighted average between the two.

\begin{tabular}{cc}
\hline Parameter & Value \\
\hline $\mathrm{n}^{\text {rim }}$ & 20 \\
$\mathrm{n}$ & 45 \\
$\mathrm{f}$ & $0.25,0.50$, and 0.75 \\
$\phi^{\text {out }}$ & $\pi / 2$ \\
$\mathrm{Hct}$ & $0.40(\mathrm{f}), 0.45(\mathrm{~m}), 0.431$ (avg) \\
$\chi_{\mathrm{do}}$ & $4 \pi \cdot 2.7 \times 10^{-7}$ \\
$\chi_{\mathrm{m}}$ & $3.4 \times 10^{-4} \mathrm{M}^{-1}$ \\
$\mathrm{Y}$ & 0.544 \\
$\mathrm{~T}_{\mathrm{E}}$ & $3.85 \mathrm{~ms}^{-1}$ \\
$\omega_{0}$ & $2 \pi \cdot 128 \mathrm{MHz}^{-1}$ \\
$\mathrm{Y}$ & $2.675 \times 10^{8} \mathrm{~T}^{-1} \mathrm{~s}^{-1}$ \\
$\mathrm{G}_{\mathrm{z}}$ & $0.5-20 \mathrm{mT} \mathrm{m}^{-1}$ \\
$\mathrm{~T}_{\mathrm{RF}}$ & $0.5-1.5 \mathrm{~ms}^{3} \mathrm{~s}^{-1}$ \\
$\mathrm{~F}$ & $3.7 \mathrm{~cm}^{3}$ \\
$\mathrm{~d}$ & $1.0 \mathrm{~cm}^{\circ}$ \\
$\theta$ & 0
\end{tabular}

$\Delta \phi_{\mathrm{PV}}$ values for the baseline, washout, and peak sections of the AIF are given in Tables 3.3 to 3.9 for $f=0.25,0.50$, and 0.75. In Tables 3.3 to 3.5, Hct values corresponding to males, females, and an average of the clinical study population are considered with the flow set to zero so that only $\Delta \phi_{\mathrm{GBCA}}$ and $\Delta \phi_{\chi}$ have an effect on the computed $\Delta \phi_{\mathrm{PV}}$ values. Tables 3.6 to 3.9 give values of $\Delta \phi_{P V}$ with $F=3.7 \mathrm{~cm}^{3} \mathrm{~s}^{-1}$ for various values of $\mathrm{G}_{\mathrm{z}}$ and $\mathrm{T}_{\mathrm{RF}}$.

\subsection{Discussion}

Equations 3.26 and 3.27 show that PVA can be expected to cause a phase error, which is additive. This conclusion is independent of the size and shape of both the ROI and the 
vessel. When $\left\langle\phi_{\mathrm{k}}^{\text {out }}\right\rangle$ can be ignored and the material immediately outside the vessel is uniform, the additive error term is given by Eq. 3.29.

For the special case where $\phi^{\text {in }}=\phi^{\text {out }}$, the phase error, $\Delta \phi_{\mathrm{PV}}$, will always be zero.

This can be seen from Eq. 3.29 since

$$
\begin{gathered}
\Delta \phi_{P V}=\frac{n^{\text {rim }}}{n}\left\{\frac{1}{n^{\text {rim }}} \sum_{k=1}^{n^{\text {rim }}} \tan ^{-1}\left[\frac{f_{k} \sin \phi^{\text {in }}+\left(1-f_{k}\right) f^{\prime} \sin \phi^{\text {in }}}{f_{k} \cos \phi^{\text {in }}+\left(1-f_{k}\right) f^{\prime} \cos \phi^{\text {in }}}\right]-\phi^{\text {in }}\right\} \\
=\frac{n^{\text {rim }}}{n}\left\{\frac{1}{n^{\text {rim }}} \sum_{k=1}^{n^{\text {rim }}} \tan ^{-1}\left[\tan \phi^{\text {in }} \frac{f_{k}+\left(1-f_{k}\right) f^{\prime}}{f_{k}+\left(1-f_{k}\right) f^{\prime}}\right]-\phi^{\text {in }}\right\} \\
=\frac{n^{\text {rim }}}{n}\left\{\frac{1}{n^{\text {rim }}} \sum_{k=1}^{n^{\text {rim }}}\left(\phi^{\text {in }}-\phi^{\text {in }}\right)\right\}=0 .
\end{gathered}
$$

However, $\phi^{\text {in }}$ and $\phi^{\text {out }}$ will not normally be equal since it was shown that both the venous blood flow during the slice selection process and the different magnetic susceptibilities between the blood and the surrounding tissues will cause these two phases to be different. Furthermore, during a DCE-MRI acquisition, the presence of a GBCA in the vessel will also affect $\phi^{\text {in }}$.

To investigate the behaviour of $\Delta \phi_{\mathrm{PV}}$, a square vessel model was considered where all of the rim-zone pixels had the same value for $f_{k}$. While this was useful for simplifying the computations, it must be remembered that such square vessels do not occur in real life. It is quite feasible to consider $f=0.0$ and $f=1.0$ in the square vessel model; however, these situations cannot occur with a circular vessel on a square pixel grid. Conversely, the behaviour of $\Delta \phi_{P V}$ for the square vessel model with $0.25 \leq f \leq 0.75$ is expected to reflect the behaviour of $\Delta \phi_{P V}$ for circular vessels with the same range of $f$ 
Table 3.3: Phase error computation for $\mathrm{Hct}=0.45$ (males) and $\mathrm{F}=0$.

\begin{tabular}{cccccccccr}
\hline $\mathrm{f}$ & $\mathrm{f}^{\prime}$ & {$[\mathrm{Gd}]$} & $\Delta \phi_{\mathrm{GBCA}}$ & $\Delta \phi_{\chi}$ & \multicolumn{4}{c}{$\Delta \phi_{\mathrm{F}}$} & \multicolumn{4}{c}{$\phi^{\text {in }}(\mathrm{rad})$} & \multicolumn{2}{c}{$\phi^{\text {in }}\left({ }^{\circ}\right)$} & $\Delta \phi_{\mathrm{PV}}(\mathrm{rad}) \Delta \phi_{\mathrm{PV}}\left(^{\circ}\right)$ \\
\hline 0.25 & 1.20 & 0.00 & 0.00 & -0.71 & 0.00 & 0.86 & 49.47 & 0.25 & 14.25 \\
0.50 & 1.20 & 0.00 & 0.00 & -0.71 & 0.00 & 0.86 & 49.47 & 0.17 & 9.86 \\
0.75 & 1.20 & 0.00 & 0.00 & -0.71 & 0.00 & 0.86 & 49.47 & 0.09 & 5.01 \\
0.25 & 0.20 & 0.50 & -0.17 & -0.71 & 0.00 & 0.69 & 39.58 & 0.14 & 8.22 \\
0.50 & 0.20 & 0.50 & -0.17 & -0.71 & 0.00 & 0.69 & 39.58 & 0.06 & 3.46 \\
0.75 & 0.20 & 0.50 & -0.17 & -0.71 & 0.00 & 0.69 & 39.58 & 0.02 & 1.25 \\
0.25 & 0.067 & 6.00 & -2.07 & -0.71 & 0.00 & -1.21 & -69.28 & 0.04 & 2.22 \\
0.50 & 0.067 & 6.00 & -2.07 & -0.71 & 0.00 & -1.21 & -69.28 & 0.01 & 0.64 \\
0.75 & 0.067 & 6.00 & -2.07 & -0.71 & 0.00 & -1.21 & -69.28 & 0.00 & 0.21 \\
\hline
\end{tabular}

Table 3.4: Phase error computation for $\mathrm{Hct}=0.40$ (females) and $\mathrm{F}=0$.

\begin{tabular}{cccccccccc}
$\mathrm{f}$ & $\mathrm{f}^{\prime}$ & {$[\mathrm{Gd}]$} & $\Delta \phi_{\mathrm{GBCA}}$ & $\Delta \phi_{\mathrm{\chi}}$ & $\Delta \phi_{\mathrm{F}}$ & $\phi^{\mathrm{in}}(\mathrm{rad})$ & $\phi^{\mathrm{in}}\left({ }^{\circ}\right)$ & $\Delta \phi_{\mathrm{PV}}(\mathrm{rad}) \Delta \phi_{\mathrm{PV}}\left({ }^{\circ}\right)$ \\
\hline 0.25 & 1.20 & 0.00 & 0.00 & -0.63 & 0.00 & 0.94 & 53.97 & 0.22 & 12.63 \\
0.50 & 1.20 & 0.00 & 0.00 & -0.63 & 0.00 & 0.94 & 53.97 & 0.15 & 8.76 \\
0.75 & 1.20 & 0.00 & 0.00 & -0.63 & 0.00 & 0.94 & 53.97 & 0.08 & 4.48 \\
0.25 & 0.20 & 0.50 & -0.17 & -0.63 & 0.00 & 0.77 & 44.08 & 0.13 & 7.52 \\
0.50 & 0.20 & 0.50 & -0.17 & -0.63 & 0.00 & 0.77 & 44.08 & 0.06 & 3.19 \\
0.75 & 0.20 & 0.50 & -0.17 & -0.63 & 0.00 & 0.77 & 44.08 & 0.02 & 1.16 \\
0.25 & 0.067 & 6.00 & -2.07 & -0.63 & 0.00 & -1.13 & -64.78 & 0.05 & 2.66 \\
0.50 & 0.067 & 6.00 & -2.07 & -0.63 & 0.00 & -1.13 & -64.78 & 0.01 & 0.77 \\
0.75 & 0.067 & 6.00 & -2.07 & -0.63 & 0.00 & -1.13 & -64.78 & 0.00 & 0.25 \\
\hline
\end{tabular}


Table 3.5: Phase error computation for $\mathrm{Hct}=0.431$ (study average) and $\mathrm{F}=0$.

\begin{tabular}{|c|c|c|c|c|c|c|c|c|c|}
\hline$f$ & $f^{\prime}$ & [Gd] & $\Delta \phi_{\mathrm{GBCA}}$ & $\Delta \phi_{\chi}$ & $\Delta \phi_{\mathrm{F}}$ & $\phi^{\mathrm{in}}(\mathrm{rad})$ & $\phi^{\text {in }}\left({ }^{\circ}\right)$ & $\Delta \phi_{\mathrm{PV}}(\mathrm{rad})$ & $\Delta \phi_{\mathrm{PV}}\left({ }^{\circ}\right)$ \\
\hline 0.25 & 1.20 & 0.00 & 0.00 & -0.68 & 0.00 & 0.89 & 51.18 & 0.24 & 13.63 \\
\hline 0.50 & 1.20 & 0.00 & 0.00 & -0.68 & 0.00 & 0.89 & 51.18 & 0.16 & 9.44 \\
\hline 0.75 & 1.20 & 0.00 & 0.00 & -0.68 & 0.00 & 0.89 & 51.18 & 0.08 & 4.81 \\
\hline 0.25 & 0.20 & 0.50 & -0.17 & -0.68 & 0.00 & 0.72 & 41.29 & 0.14 & 7.96 \\
\hline 0.50 & 0.20 & 0.50 & -0.17 & -0.68 & 0.00 & 0.72 & 41.29 & 0.06 & 3.36 \\
\hline 0.75 & 0.20 & 0.50 & -0.17 & -0.68 & 0.00 & 0.72 & 41.29 & 0.02 & 1.22 \\
\hline 0.25 & 0.067 & 6.00 & -2.07 & -0.68 & & -1.18 & & 0.04 & 2.39 \\
\hline 0.50 & 0.067 & 6.00 & -2.07 & -0.68 & 0.00 & -1.18 & -67.57 & 0.01 & 0.69 \\
\hline 0.75 & 0.067 & 6.00 & -2.07 & -0.68 & 0.00 & -1.18 & -67.57 & 0.00 & 0.22 \\
\hline
\end{tabular}

Table 3.6: Phase error computation for $\mathrm{Hct}=0.431$ (study average), $\mathrm{F}=3.7 \mathrm{~cm}^{3} \mathrm{~s}^{-1}, \mathrm{G}_{\mathrm{z}}=$ $1 \mathrm{mT} \mathrm{m}^{-1}$, and $\mathrm{T}_{\mathrm{RF}}=1 \mathrm{~ms}$.

\begin{tabular}{cccccccccr}
\hline $\mathrm{f}$ & $\mathrm{f}^{\prime}$ & {$[\mathrm{Gd}]$} & $\Delta \phi_{\mathrm{GBCA}}$ & $\Delta \phi_{\mathrm{X}}$ & $\Delta \phi_{\mathrm{F}}$ & $\phi^{\mathrm{in}}(\mathrm{rad})$ & \multicolumn{4}{c}{$\phi^{\mathrm{in}}\left(^{\circ}\right)$} & $\Delta \phi_{\mathrm{PV}}(\mathrm{rad}) \Delta \phi_{\mathrm{PV}}\left({ }^{\circ}\right)$ \\
\hline 0.25 & 1.20 & 0.00 & 0.00 & -0.68 & -0.006 & 0.89 & 50.82 & 0.24 & 13.76 \\
0.50 & 1.20 & 0.00 & 0.00 & -0.68 & -0.006 & 0.89 & 50.82 & 0.17 & 9.53 \\
0.75 & 1.20 & 0.00 & 0.00 & -0.68 & -0.006 & 0.89 & 50.82 & 0.08 & 4.85 \\
0.25 & 0.20 & 0.50 & -0.17 & -0.68 & -0.006 & 0.71 & 40.93 & 0.14 & 8.01 \\
0.50 & 0.20 & 0.50 & -0.17 & -0.68 & -0.006 & 0.71 & 40.93 & 0.06 & 3.38 \\
0.75 & 0.20 & 0.50 & -0.17 & -0.68 & -0.006 & 0.71 & 40.93 & 0.02 & 1.23 \\
0.25 & 0.067 & 6.00 & -2.07 & -0.68 & -0.006 & -1.19 & -67.93 & 0.04 & 2.36 \\
0.50 & 0.067 & 6.00 & -2.07 & -0.68 & -0.006 & -1.19 & -67.93 & 0.01 & 0.68 \\
0.75 & 0.067 & 6.00 & -2.07 & -0.68 & -0.006 & -1.19 & -67.93 & 0.00 & 0.22 \\
\hline
\end{tabular}


Table 3.7: Phase error computation for $\mathrm{Hct}=0.431$ (study average), $\mathrm{F}=3.7 \mathrm{~cm}^{3} \mathrm{~s}^{-1}, \mathrm{G}_{\mathrm{z}}=$ $10 \mathrm{mT} \mathrm{m}^{-1}$, and $\mathrm{T}_{\mathrm{RF}}=1.5 \mathrm{~ms}$.

\begin{tabular}{cccccccccr}
\hline $\mathrm{f}$ & $\mathrm{f}^{\prime}$ & {$[\mathrm{Gd}]$} & $\Delta \phi_{\mathrm{GBCA}}$ & $\Delta \phi_{\mathrm{X}}$ & $\Delta \phi_{\mathrm{F}}$ & $\phi^{\mathrm{in}}(\mathrm{rad})$ & \multicolumn{4}{c}{$\phi^{\mathrm{in}}\left(^{\circ}\right)$} & $\Delta \phi_{\mathrm{PV}}(\mathrm{rad}) \Delta \phi_{\mathrm{PV}}\left({ }^{\circ}\right)$ \\
\hline 0.25 & 1.20 & 0.00 & 0.00 & -0.68 & -0.142 & 0.75 & 43.06 & 0.29 & 16.56 \\
0.50 & 1.20 & 0.00 & 0.00 & -0.68 & -0.142 & 0.75 & 43.06 & 0.20 & 11.44 \\
0.75 & 1.20 & 0.00 & 0.00 & -0.68 & -0.142 & 0.75 & 43.06 & 0.10 & 5.75 \\
0.25 & 0.20 & 0.50 & -0.17 & -0.68 & -0.142 & 0.58 & 33.16 & 0.16 & 9.21 \\
0.50 & 0.20 & 0.50 & -0.17 & -0.68 & -0.142 & 0.58 & 33.16 & 0.07 & 3.81 \\
0.75 & 0.20 & 0.50 & -0.17 & -0.68 & -0.142 & 0.58 & 33.16 & 0.02 & 1.37 \\
0.25 & 0.067 & 6.00 & -2.07 & -0.68 & -0.142 & -1.32 & -75.69 & 0.03 & 1.57 \\
0.50 & 0.067 & 6.00 & -2.07 & -0.68 & -0.142 & -1.32 & -75.69 & 0.01 & 0.45 \\
0.75 & 0.067 & 6.00 & -2.07 & -0.68 & -0.142 & -1.32 & -75.69 & 0.00 & 0.14 \\
\hline
\end{tabular}

Table 3.8: Phase error computation for $\mathrm{Hct}=0.431$ (study average), $\mathrm{F}=3.7 \mathrm{~cm}^{3} \mathrm{~s}^{-1}, \mathrm{G}_{\mathrm{z}}=$ $20 \mathrm{mT} \mathrm{m}^{-1}$, and $\mathrm{T}_{\mathrm{RF}}=1.5 \mathrm{~ms}$.

\begin{tabular}{cccccccccr}
\hline $\mathrm{f}$ & $\mathrm{f}^{\prime}$ & {$[\mathrm{Gd}]$} & $\Delta \phi_{\mathrm{GBCA}}$ & $\Delta \phi_{\mathrm{X}}$ & $\Delta \phi_{\mathrm{F}}$ & $\phi^{\mathrm{in}}(\mathrm{rad})$ & \multicolumn{4}{c}{$\phi^{\mathrm{in}}\left(^{\circ}\right)$} & $\Delta \phi_{\mathrm{PV}}(\mathrm{rad}) \Delta \phi_{\mathrm{PV}}\left({ }^{\circ}\right)$ \\
\hline 0.25 & 1.20 & 0.00 & 0.00 & -0.68 & -0.284 & 0.61 & 34.94 & 0.34 & 19.53 \\
0.50 & 1.20 & 0.00 & 0.00 & -0.68 & -0.284 & 0.61 & 34.94 & 0.23 & 13.44 \\
0.75 & 1.20 & 0.00 & 0.00 & -0.68 & -0.284 & 0.61 & 34.94 & 0.12 & 6.64 \\
0.25 & 0.20 & 0.50 & -0.17 & -0.68 & -0.284 & 0.44 & 25.04 & 0.18 & 10.42 \\
0.50 & 0.20 & 0.50 & -0.17 & -0.68 & -0.284 & 0.44 & 25.04 & 0.07 & 4.22 \\
0.75 & 0.20 & 0.50 & -0.17 & -0.68 & -0.284 & 0.44 & 25.04 & 0.03 & 1.49 \\
0.25 & 0.067 & 6.00 & -2.07 & -0.68 & -0.284 & -1.46 & -83.82 & 0.01 & 0.69 \\
0.50 & 0.067 & 6.00 & -2.07 & -0.68 & -0.284 & -1.46 & -83.82 & 0.00 & 0.20 \\
0.75 & 0.067 & 6.00 & -2.07 & -0.68 & -0.284 & -1.46 & -83.82 & 0.00 & 0.06 \\
\hline
\end{tabular}


Table 3.9: Summary of the baseline and washout phases ( $\phi_{\mathrm{b}}$ and $\phi_{\mathrm{w}}$, respectively) and the difference between the two for $f=0.5, F=3.7 \mathrm{~cm}^{3} \mathrm{~s}^{-1}$, Hct $=0.431$, and various input parameters.

\begin{tabular}{ccccc}
\hline $\mathrm{T}_{\mathrm{RF}}(\mathrm{ms})$ & $\mathrm{G}_{\mathrm{z}}\left(\mathrm{mT} \mathrm{m}^{-1}\right)$ & $\phi_{\mathrm{b}}\left({ }^{\circ}\right)$ & $\phi_{\mathrm{w}}\left({ }^{\circ}\right)$ & $\phi_{\mathrm{b}}-\phi_{\mathrm{w}}\left(^{\circ}\right)$ \\
\hline 0.0 & 0.0 & 9.44 & 3.36 & 6.08 \\
0.5 & 0.5 & 9.45 & 3.36 & 6.09 \\
1.0 & 1.0 & 9.53 & 3.38 & 6.15 \\
\hline 0.5 & 10.0 & 9.66 & 3.41 & 6.25 \\
\hline 1.0 & 10.0 & 10.33 & 3.57 & 6.76 \\
1.0 & 15.0 & 10.77 & 3.67 & 7.10 \\
1.0 & 20.0 & 11.21 & 3.77 & 7.44 \\
\hline 1.5 & 10.0 & 11.44 & 3.81 & 7.63 \\
1.5 & 15.0 & 12.44 & 4.02 & 8.42 \\
1.5 & 20.0 & 13.44 & 4.22 & 9.22 \\
\hline
\end{tabular}

values.

To investigate the behaviour of $\Delta \phi_{\mathrm{PV}}$, a square vessel model was considered where all of the rim-zone pixels had the same value for $f_{k}$. While this was useful for simplifying the computations, it must be remembered that such square vessels do not occur in real life. It is quite feasible to consider $f=0.0$ and $f=1.0$ in the square vessel model; however, these situations cannot occur with a circular vessel on a square pixel grid. Conversely, the behaviour of $\Delta \phi_{P V}$ for the square vessel model with $0.25 \leq f \leq 0.75$ is expected to reflect the behaviour of $\Delta \phi_{P V}$ for circular vessels with the same range of $f$ values.

When considering the phase error in the context of AIF measurements, it is important to note the $\Delta \phi_{\mathrm{PV}}$ value changes with the magnitude of the signal inside the 
vessel. As this signal magnitude increases, $\mathrm{f}^{\prime}$ decreases. It can be seen from Eq. 3.29 that, in the limit of the intra-vessel signal magnitude becoming infinitely large (i.e. $f^{\prime} \rightarrow 0$ ), $\Delta \Phi_{P V}$ goes to zero. This suggests that the phase error will be largest for the AIF baseline, will be quite small for the peak, and will have a value between these extremes for the washout. This trend can also be seen consistently in Tables 3.3-3.6 where $f^{\prime}$ values of $1.2,0.2$, and 0.067 are considered to correspond to the baseline, washout, and peak, respectively.

From Tables 3.6 to 3.9, it can be seen that the phase error increases with both $G_{z}$ and $\mathrm{T}_{\mathrm{RF}}$ from fairly small values to very significant errors. Thus, the phase error for a given measurement will depend strongly on the details of the sequence used. Unfortunately, $\mathrm{G}_{\mathrm{z}}$ and $\mathrm{T}_{\mathrm{RF}}$ values are not readily available to the scanner operator. The contribution to the phase error from $\Delta \chi$ also depends on a sequence parameter, $T_{E}$, but this value is adjustable by the scanner operator. For the measurements reported later in the thesis, $\mathrm{T}_{\mathrm{E}}$ was always set to $3.85 \mathrm{~ms}$ and the $\Delta \phi_{\mathrm{PV}}$ values given in Tables 3.3 to 3.9 were all calculated with this $T_{E}$ value.

The $\Delta \phi_{\mathrm{PV}}$ values caused by $\Delta \chi$ have a significant dependence on the amount of Hct and the oxygen saturation level of the blood. Tables 3.3 to 3.9 give $\Delta \phi_{\mathrm{PV}}$ values for an average resting state oxygen saturation of $Y=0.544$ [30] and Hct values of 0.45 (for males), 0.40 (for females), and an average of these two values. It can be seen from the tables that for the baseline (i.e. $f^{\prime}=1.2$ ) and the washout (i.e. $f^{\prime}=0.2$ ), this contribution to $\Delta \phi_{\mathrm{PV}}$ is appreciable.

The values of $\mathrm{G}_{\mathrm{z}}$ and $\mathrm{T}_{\mathrm{RF}}$ used to compute the $\Delta \phi_{\mathrm{PV}}$ values given in Tables 3.6 to 3.9 were chosen to demonstrate that blood flow during slice selection can lead to 
reasonably large phase shifts. However, for the 3D FLASH sequence used for the AIF measurements reported in this thesis, the values of $\mathrm{G}_{\mathrm{z}}$ and $\mathrm{T}_{\mathrm{RF}}$ are likely less than $1.0 \mathrm{mT}$ $\mathrm{m}^{-1}$ and $1.0 \mathrm{~ms}$, respectively, although the exact values are difficult to determine. Consequently, the contribution to $\Delta \phi_{\mathrm{PV}}$ from $\Delta \phi_{\mathrm{F}}$ is expected to be quite small compared to the contribution from $\Delta \phi_{\chi}$.

If the contributions to $\Delta \phi$ from $\Delta \chi$ are considered to be the dominant ones if the behaviour of the $\Delta \phi_{\mathrm{PV}}$ values for $f=0.5$ are considered to be a reasonable representation of what might happen in the real world, it can be seen from Table 3.5 that phase errors of about $9^{\circ}, 3^{\circ}$, and $1^{\circ}$ can be expected for the baseline $\left(\phi_{b}\right)$, washout $\left(\phi_{w}\right)$, and peak, respectively. The average difference between the baseline and washout values for $f=0.5$ is about $6^{\circ}$. From Table 3.9, it can be seen that this difference increases as $G_{z}$ and $T_{R F}$ increase. 


\section{Chapter 4}

\section{The Phase and $\mathrm{T}_{1}$ Arterial Input Function}

\subsection{Introduction}

In the previous chapter, issues associated with the current methods used to acquire the AIF in DCE-MRI were discussed. In particular, the effect of partial volume averaging (PVA) on the signal phase was discussed in some detail in Section 3.3 where an equation for the phase error caused by PVA, $\Delta \phi_{\mathrm{PV}}$, was derived (Eq. 3.30). This equation shows that $\Delta \phi_{\mathrm{PV}}$ is a function of both $\phi^{\text {in }}$ and $\phi^{\text {out }}$, the phases inside and outside of the vessel, respectively. In Section 3.4 it was argued that it is reasonable to assume that $\phi^{\text {out }}=\pi / 2$ for all situations being considered here. It was also shown that $\phi^{\text {in }}$ is affected by: (i) the difference in magnetic susceptibility between the inside of the vessel and the outside $\left(\Delta \phi_{\chi}\right)$; (ii) motion of the nuclear spins during the slice selection process $\left(\Delta \phi_{F}\right)$; and (iii) the

presence of a GBCA inside the vessel $\left(\Delta \phi_{\mathrm{GBCA}}\right)$. Equations showing the contributions to $\Delta \phi_{\mathrm{PV}}$ from $\Delta \phi_{\chi^{\prime}} \Delta \phi_{\mathrm{F}}$, and $\Delta \phi_{\mathrm{GBCA}}$ are given in Eqs. 3.38, 3.48, and 3.49, respectively. 
In Section 3.5, parameter values that are relevant to the experimental work given later in the thesis were used to compute $\Delta \phi_{\mathrm{PV}}$ values. These were summarized in Tables 3.3-3.9. One important observation from these results is that $\Delta \phi_{\mathrm{PV}}$ decreases with increasing [Gd]. In particular, the phase error for the baseline phase is considerably higher than for the washout; for $f=0.5$ it is about three times higher. This is unfortunate since, when the phase is used to obtain the AIF, the baseline phase (along with its relatively large phase error) is subtracted from each data point to get the final AIF. Based on this observation, a novel approach for the acquisition of the AIF is developed that is more accurate than the currently used method. This work is presented in Section 4.2.

With the new method, the AIF is calculated from phase changes relative to the washout value rather than the baseline phase and $T_{1}$ relaxation information is also incorporated. The AIF measured with the traditional method will be referred to here as the "Phase-Only" (PO) AIF while the AIF obtained with the new approach will be called the "Phase and $\mathrm{T}_{1}$ " (PT) AIF.

In Section 4.4 the results of computer simulations designed to further investigate the effect of phase experimental error on the AIF are presented. The goal of these simulations was to further demonstrate that the PT AIF is superior to the PO AIF, as well as to gain a better understanding of why this is the case.

When an AIF measurement is performed on a patient as part of a DCE-MRI acquisition, an intravenous injection of a GBCA is given to the patient. The AlF is the timedependence of the concentration of this GBCA ([Gd]) in the blood at a specific location in an artery feeding the tissue of interest. To simulate the AIF measurement, it is necessary 
to have knowledge of the $[G d]$ as a function of time, $[G d](t)$, since this is an important part of the real procedure. The $[\mathrm{Gd}](\mathrm{t})$ function for the bolus injection in the simulations was obtained by using a known AIF from the literature [67]. Thus, in the absence of experimental error in the simulations the resulting simulated AIF should be identical to the assumed input AIF that was used as the $[\mathrm{Gd}](\mathrm{t})$ function. When experimental error is inserted into the simulations, it is expected that the simulated AIF will deviate from the input function. These simulations were used to investigate the effect of several sources of phase error on the computed AIF.

The AIF used for the simulations presented here was taken from the work of Parker et al. [67]. The more recently published AIF given by Georgiou et al. [68], which is based on the functional form given by Horsfield et al. [69], could also have been used. However, the details of the assumed input are less important than the performance of the simulated measurement in reproducing this input. A useful extension of the work presented in this chapter would be to consider the AIF from Georgiou et al. [68].

\section{2 "Phase-Only" AIF and "Phase and $\mathrm{T}_{1}$ " AIF}

\subsubsection{PO AIF}

Equation 2.109 can be rewritten as a time-dependent AIF function:

$$
[\mathrm{Gd}](\mathrm{t})=\operatorname{AIF}(\mathrm{t})=\mathrm{K}\left[\phi(\mathrm{t})-\phi_{0}\right]=\mathrm{K} \Delta \phi
$$

where $\Delta \phi$ is the difference in $\phi$ measured at time $\mathrm{t}$ from the baseline $\phi_{0}$ value with no GBCA present, and $\mathrm{K}$ is a constant determined by the conditions of the DCE-MRI 
acquisition:

$$
K=\frac{-1}{\omega_{0} X_{m} T_{E} f(\theta)}
$$

In this work, an AIF computed from Eq. 4.1 (i.e. from $\phi(t)$ alone) is referred to as the 'Phase-Only' (PO) AIF.

One of the main challenges in obtaining the PO AIF is the acquisition of accurate and precise values for $\phi_{0}$. When the MR signal magnitude is high (e.g. around the AIF peak) the uncertainty of the signal phase is low. However, when the signal magnitude is low (e.g. AIF baseline) the signal phase is prone to experimental error $[30,58]$. This effect is shown in Fig. 4.1. Unfortunately, these higher uncertainties for the $\phi_{0}$ measurements affect the entire PO AIF curve since $\phi_{0}$ is used to calculate every point on the AIF curve (Eq. 4.1). This can lead to inaccuracies in DCE-MRI parameters subsequently computed using the PO AIF. The nature of these errors then depends on how the measured AIF differs from the true AIF. If the area under the measured AIF curve is too low (as is the case when the magnitude only approach is used), this can be interpreted as a lower input volume of blood leading to an overestimate of quantitative DCE-MRI parameter values in normal brain tissue [71]. Note that these AIF characteristics will have important effects on dynamic susceptibility contrast (DSC)-MRI measurements [54] but less of an effect on DCE-MRI parameter values. These effects have not been studied carefully for situations such as in gliomas, where the blood brain barrier has deteriorated, but it can be expected that there will be similar effects.

In a blood vessel, errors in $\phi(t)$ due to PVA effects are greatly reduced after GBCA injection when the signal magnitude in the vessel dwarfs that of surrounding tissues [30]. 


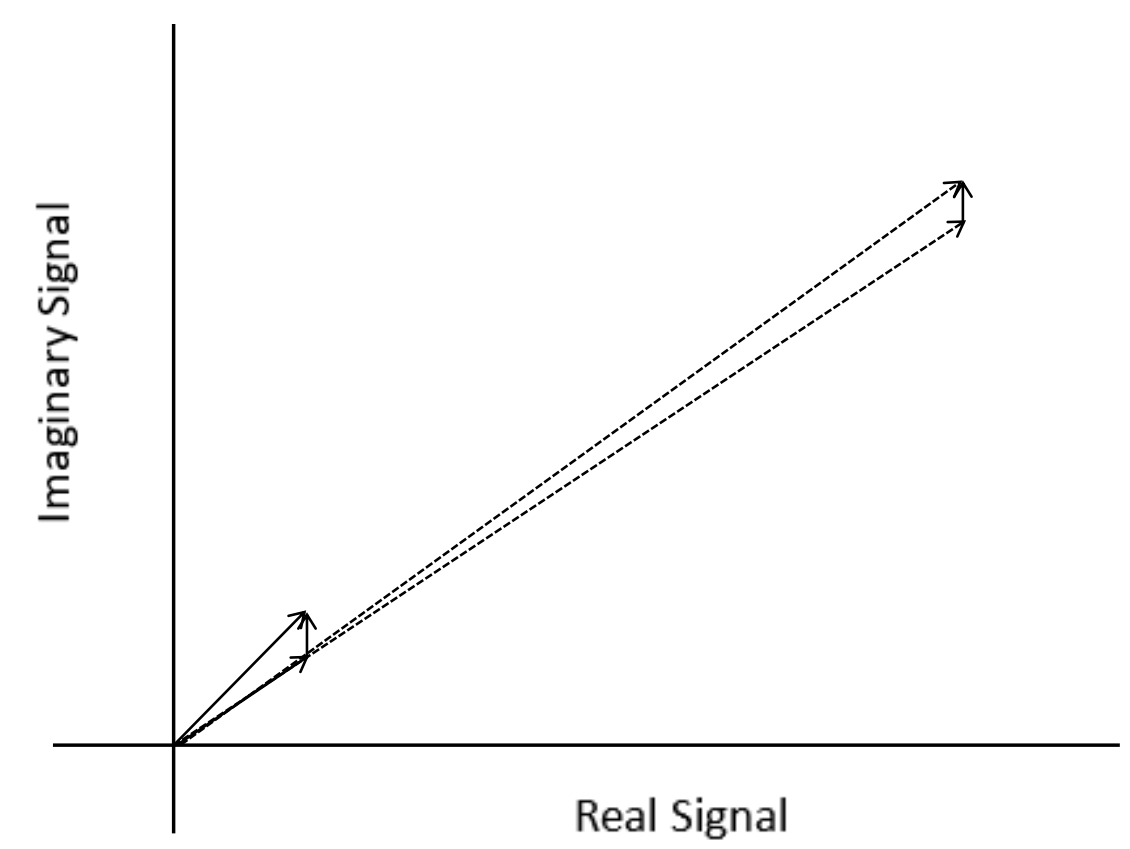

Figure 4.1: The difference in acquired MR phase based on a constant experimental error (shown with the vertical arrow). For low signal magnitude data (the solid arrows), the phase uncertainty is much higher than in high signal magnitude data (the dashed arrows).

For a large vascular signal, the phase angle of the vector sum of that signal and a small extravascular contribution will only be affected significantly when the vascular signal magnitude is small compared with the extravascular signal magnitude. Since the intravascular signal magnitude is significantly higher during the washout portion of the AIF curve than at the baseline, it follows that the phase during the washout portion of the curve, $\phi_{\mathrm{w}}$, can be measured more reliably than $\phi_{0}$. Note that this effect is independent of both the actual phase angle and the difference from the baseline phase value. In the following section, a method to compute the AlF is derived combining $\phi$ and $T_{1}$ measurements. 


\subsubsection{PT AIF Derivation}

The $[\mathrm{Gd}]$ values in the washout portion of the AIF curve can be considered to be timeindependent on the time scale of a DCE-MRI measurement. Therefore, the AIF washout value, AlF $_{w}$, can be defined, from Eq. 4.1 as

$$
\mathrm{AlF}_{\mathrm{w}} \equiv \mathrm{K}\left[\phi_{\mathrm{w}}-\phi_{0}\right]
$$

where $\phi_{w^{\prime}}$ the phase of the MRI signal during the washout, will is also be timeindependent.

If an accurate AlF $_{w}$ value (measured independently of the bulk of the AlF acquisition) is available, another equation for the full AIF can be obtained from Eq. 4.1 by adding and subtracting AlF $_{\mathrm{w}}$ :

$$
\begin{aligned}
\operatorname{AIF}(t) & =K\left[\phi(t)-\phi_{0}\right]-A_{\mathrm{IIF}}+\mathrm{AIF}_{\mathrm{w}} \\
& =\mathrm{K}\left[\phi(\mathrm{t})-\phi_{0}-\phi_{\mathrm{w}}+\phi_{0}\right]+\mathrm{AIF}_{\mathrm{w}} \\
& =\mathrm{K}\left[\phi(\mathrm{t})-\phi_{\mathrm{w}}\right]+\mathrm{AIF}_{\mathrm{w}} .
\end{aligned}
$$

It is proposed below that this accurate $A F_{w}$ value can be obtained using $T_{1}$ measurements. With this new strategy, the AIF is calculated from GBCA-induced phase differences relative to $\phi_{\mathrm{w}}$, rather than $\phi_{0}$, eliminating the troublesome $\phi_{0}$ from the computation altogether. AlFs computed using Eq. 4.4 will be referred to as "Phase and $\mathrm{T}_{1}^{\prime \prime}(\mathrm{PT})$ AlFs.

Explicitly incorporating experimental error into the AIF functions gives:

$$
\operatorname{aif}^{\mathrm{po}}(\mathrm{t})=\mathrm{K}\left[\phi(\mathrm{t})+\delta \phi(\mathrm{t})-\left(\phi_{0}+\delta \phi_{0}(\mathrm{t})\right)\right]
$$

and

$$
\operatorname{aif}^{\mathrm{pt}}(\mathrm{t})=\mathrm{K}\left[\phi(\mathrm{t})+\delta \phi(\mathrm{t})-\left(\phi_{\mathrm{w}}+\delta \phi_{\mathrm{w}}(\mathrm{t})\right)\right]+\mathrm{AIF}_{\mathrm{w}}
$$


which correspond to the PO and PT AIFs, respectively. The terms $\delta \phi_{0}(t), \delta \phi(t)$, and $\delta \phi_{w}(t)$ are the experimental errors associated with the measurements of $\phi_{0}, \phi(t)$, and $\phi_{w^{\prime}}$ respectively. It is assumed that these experimental errors are additive, are uncorrelated, and that they are equally likely to be positive or negative. It is also assumed that $\phi(t)$ and $\delta \phi(t)$ are uncorrelated and that the $A_{\mathrm{w}}$ value obtained with another method (e.g. from $\mathrm{T}_{1}$ measurements) does not contribute appreciably to the experimental error.

In Eqs. 4.5 and 4.6 an explicit time-dependence was included for $\delta \phi_{0}$ and $\delta \phi_{w^{\prime}}$ even though $\phi_{0}$ and $\phi_{\mathrm{w}}$ are time-independent, since different measurements are not expected to yield identical results. However, these time-dependent contributions will mainly be due to Gaussian random noise with a mean of zero, so that a time average over enough data points will cause these contributions to approach zero. In what follows, it will be assumed that either: (i) both $\phi_{0}$ and $\phi_{\mathrm{w}}$ are averaged over enough measurements that these random fluctuations can be ignored; or (ii) these fluctuations are small compared with the other time-dependent contributions to experimental error. Based on this assumption, $\delta \phi_{0}$ and $\delta \phi_{\mathrm{w}}$ will both be considered to be time-independent for the rest of the thesis.

From Eqs. 4.1 and 4.5, it can readily be seen that aif ${ }^{\mathrm{po}}(\mathrm{t})$ can be written in terms of $\operatorname{AIF}(\mathrm{t})$ as

$$
\operatorname{aif}^{\mathrm{po}}(\mathrm{t})=\operatorname{AIF}(\mathrm{t})+\mathrm{K}\left[\delta \phi(\mathrm{t})-\delta \phi_{0}\right]
$$

This shows that the experimental error for the aif ${ }^{\mathrm{po}}(\mathrm{t})$ measurement at a time $t$ is given by the time-dependent term $\mathrm{K}\left[\delta \phi(\mathrm{t})-\delta \phi_{0}\right]$. For the washout, this can be written as

$$
\operatorname{aif}_{\mathrm{w}}^{\mathrm{po}}=\mathrm{AIF}_{\mathrm{w}}+\mathrm{K}\left[\delta \phi_{\mathrm{w}}-\delta \phi_{0}\right]
$$


where the second term is the time-independent experimental error for the washout. The difference between the true washout value (which is known) and the measured value is equal to the constant term $\mathrm{K}\left[\delta \phi_{\mathrm{w}}-\delta \phi_{0}\right]$.

Consider what happens when this term is subtracted from each point of aif ${ }^{\mathrm{po}}(\mathrm{t})$. This gives:

$$
\begin{gathered}
\operatorname{aif}^{\mathrm{po}}(\mathrm{t})-\mathrm{K}\left[\delta \phi_{\mathrm{w}}-\delta \phi_{0}\right]=\mathrm{K}\left[\phi(\mathrm{t})+\delta \phi(\mathrm{t})-\phi_{0}-\delta \phi_{0}-\delta \phi_{\mathrm{w}}+\delta \phi_{0}\right] \\
=\mathrm{K}\left[\phi(\mathrm{t})+\delta \phi(\mathrm{t})-\phi_{0}-\delta \phi_{\mathrm{w}}\right] .
\end{gathered}
$$

Now add and subtract AIF $_{\mathrm{w}}$ :

$$
\operatorname{aif}^{\mathrm{po}}(\mathrm{t})-\mathrm{K}\left[\delta \phi_{\mathrm{w}}-\delta \phi_{0}\right]=\mathrm{K}\left[\phi(\mathrm{t})+\delta \phi(\mathrm{t})-\phi_{0}-\delta \phi_{\mathrm{w}}\right]-\mathrm{AIF}_{\mathrm{w}}+\mathrm{AIF}_{\mathrm{w}}
$$

which, using Eq. 4.3, can be written as

$$
\begin{aligned}
\operatorname{aif}^{\mathrm{po}}(\mathrm{t})-\mathrm{K}\left[\delta \phi_{\mathrm{w}}-\delta \phi_{0}\right] & =\mathrm{K}\left[\phi(\mathrm{t})+\delta \phi(\mathrm{t})-\phi_{0}-\delta \phi_{\mathrm{w}}-\left(\phi_{\mathrm{w}}-\phi_{0}\right)\right]+\mathrm{AlF}_{\mathrm{w}} \\
& =\mathrm{K}\left[\phi(\mathrm{t})+\delta \phi(\mathrm{t})-\left(\phi_{\mathrm{w}}+\delta \phi_{\mathrm{w}}\right)\right]+\mathrm{AlF}_{\mathrm{w}} \\
& =\operatorname{aif}^{\mathrm{pt}}(\mathrm{t})
\end{aligned}
$$

Thus, the two 'aif' functions can be seen to differ only by an additive constant. In other words, if the whole aif ${ }^{\mathrm{po}}(\mathrm{t})$ curve is "shifted vertically" until the washout value is equal to the accurate value obtained independently, the aif ${ }^{\mathrm{pt}}(\mathrm{t})$ curve is obtained.

From Eqs. 4.5 and 4.6, the total experimental errors for aif ${ }^{\mathrm{po}}(\mathrm{t})$ and aif ${ }^{\mathrm{pt}}(\mathrm{t})$ are given by $K\left[\delta \phi(t)-\delta \phi_{0}\right]$ and $K\left[\delta \phi(t)-\delta \phi_{w}\right]$, respectively. aif ${ }^{\mathrm{po}}(0)$ will equal zero (as it must) even though $\delta \phi_{0}$ may be large since the error is explicitly subtracted away. However, all other points on the curve will be shifted from their true values. Conversely, Eq. 4.6 inherently gives the correct value for the washout of aif ${ }^{\mathrm{pt}}(\mathrm{t})$ but, in general, aif $^{\mathrm{pt}}(0)$ will not equal zero. At the peak it can easily be seen that aif ${ }^{\mathrm{pt}}(\mathrm{t})$ is more accurate 
than aif ${ }^{\mathrm{po}}(\mathrm{t})$ since $\delta \phi_{\mathrm{w}}<\delta \phi_{0}$ and $\delta \phi(\mathrm{t})$ will be very small compared $\delta \phi_{\mathrm{w}}$. This will also be true for the rest of the curve (with the exception of $t=0$ ), but to a lesser extent. Note that the true baseline value for the AIF is known to be zero but the measured value, aif $^{\mathrm{pt}}(0)$ differs from zero by a term that contains only experimental error. This source of error can be removed by simply resetting the baseline value to zero.

The accurate AlF $_{\mathrm{w}}$ required for Eqs. 4.4 and 4.6 can be determined from the change in $T_{1}$ caused by the presence of a GBCA by rearranging Eq. 2.84 to get:

$$
\operatorname{AIF}_{\mathrm{w}}=[\mathrm{Gd}](\text { late time })=\frac{1}{\mathrm{r}_{1}}\left[\frac{1}{\mathrm{~T}_{1, \mathrm{w}}}-\frac{1}{\mathrm{~T}_{1,0}}\right]
$$

where $T_{1, w}$ is the measured $T_{1}$ value obtained during the washout and $r_{1}$ is the relaxivity of the contrast agent. It is not practical to perform accurate $T_{1}$ measurements fast enough to properly characterize the entire AIF in this way; however, it is feasible to obtain an accurate $\left[G d\right.$ ] value for the washout based on careful $T_{1,0}$ and $T_{1, w}$ measurements.

\subsection{Simulation Methods}

\subsubsection{Parker AIF}

To observe how sources of error in clinical imaging can affect the measurement of an AIF, noise and PVA effects were simulated to occur for AIFs based on 'errors' applied to the complex MR signal (S). A known AIF was used to investigate these effects. The AIF chosen for this work was computed from the functional fit to 67 averaged, clinically measured AIFs as given by Parker et al. [67]. Their DCE-MRI data were acquired using a series of $\mathrm{T}_{1^{-}}{ }^{-}$ weighted spoiled gradient echo (GRE) sequences on a 1.5 T Philips Intera MRI system 
(Philips, Best, Netherlands) with the following parameters: repetition time $\left(T_{R}\right)=4.0 \mathrm{~ms}$, echo time $\left(T_{E}\right)=0.82 \mathrm{~ms}, 75$ consecutively acquired axial volumes, flip angle $(\alpha)=20^{\circ}$, temporal resolution $=4.97 \mathrm{~s}, 25$ slices acquired with overcontiguous slice spacing to create a slice thickness of $4 \mathrm{~mm}$ for small lesions and $8 \mathrm{~mm}$ for large lesions, FOV = $375 \times 375 \mathrm{~mm}^{2}$, and the in-plane resolution was $128 \times 128$. The measured AIFs were obtained from changes in SI as the bolus of GBCA passed through either the descending aorta or iliac arteries, depending on the lesion type. What will be referred to here as the 'Parker AIF', or AIF , was constructed by fitting the average measured AIF to the sum of two Gaussian functions and an exponential function modulated by a sigmoid function [67]:

$$
\operatorname{AIF}_{\mathrm{p}}(\mathrm{t})=\sum_{r=1}^{2} \frac{\mathrm{A}_{\mathrm{r}}}{\sigma_{r} \sqrt{2 \pi}} \cdot \exp \left(-\frac{\left(\mathrm{t}-\lambda_{\mathrm{r}}\right)^{2}}{2 \sigma_{\mathrm{r}}^{2}}\right)+\frac{v \mathrm{e}^{-\beta \mathrm{t}}}{1+\exp (-\mathrm{s}(\mathrm{t}-\tau))}
$$

Variables $A_{r}, \lambda_{r}$, and $\sigma_{r}$ are scaling constants, centers, and standard deviations, respectively, for each Gaussian; $v$ is the amplitude and $\beta$ is the decay constant of the exponential; and $s$ and $\tau$ are the width and center of the sigmoid, respectively. The parameter values used to create AIF $_{\mathrm{P}}$ using Eq. 4.13 are given in Table 4.1 and the constructed AIF is shown in Fig. 4.2. AlF $\mathrm{P}_{\mathrm{P}}$ was shifted in time so that the peak value occurred about two minutes after the start of the acquisition to match the time at which the peak value of the AIF obtained for the dynamic phantom (described in Section 5.2.3) occurred. 
Table 4.1: Parameters and standard deviation (SD) values used to create AlF $_{\mathrm{P}}$ (Eq. 4.13), as given by Parker et al. [67].

\begin{tabular}{c|cccccccccc}
\hline Parameter & $\mathrm{A}_{1}$ & $\mathrm{~A}_{2}$ & $\lambda_{1}$ & $\lambda_{2}$ & $\sigma_{1}$ & $\sigma_{2}$ & $\mathrm{v}$ & $\beta$ & $\mathrm{s}$ & $\tau$ \\
\cline { 2 - 10 } Value & 0.809 & 0.330 & 0.171 & 0.365 & 0.056 & 0.132 & 1.050 & 0.169 & 38.08 & 0.483 \\
SD & 0.044 & 0.040 & 0.001 & 0.028 & 0.001 & 0.021 & 0.017 & 0.006 & 16.78 & 0.015 \\
Units & $\mathrm{mmol} \min$ & $\mathrm{mmol} \mathrm{min}$ & $\mathrm{min}$ & $\min$ & $\min$ & $\min$ & $\mathrm{mmol}$ & $\mathrm{min}^{-1}$ & $\mathrm{~min}^{-1}$ & $\mathrm{~min}$ \\
\hline
\end{tabular}

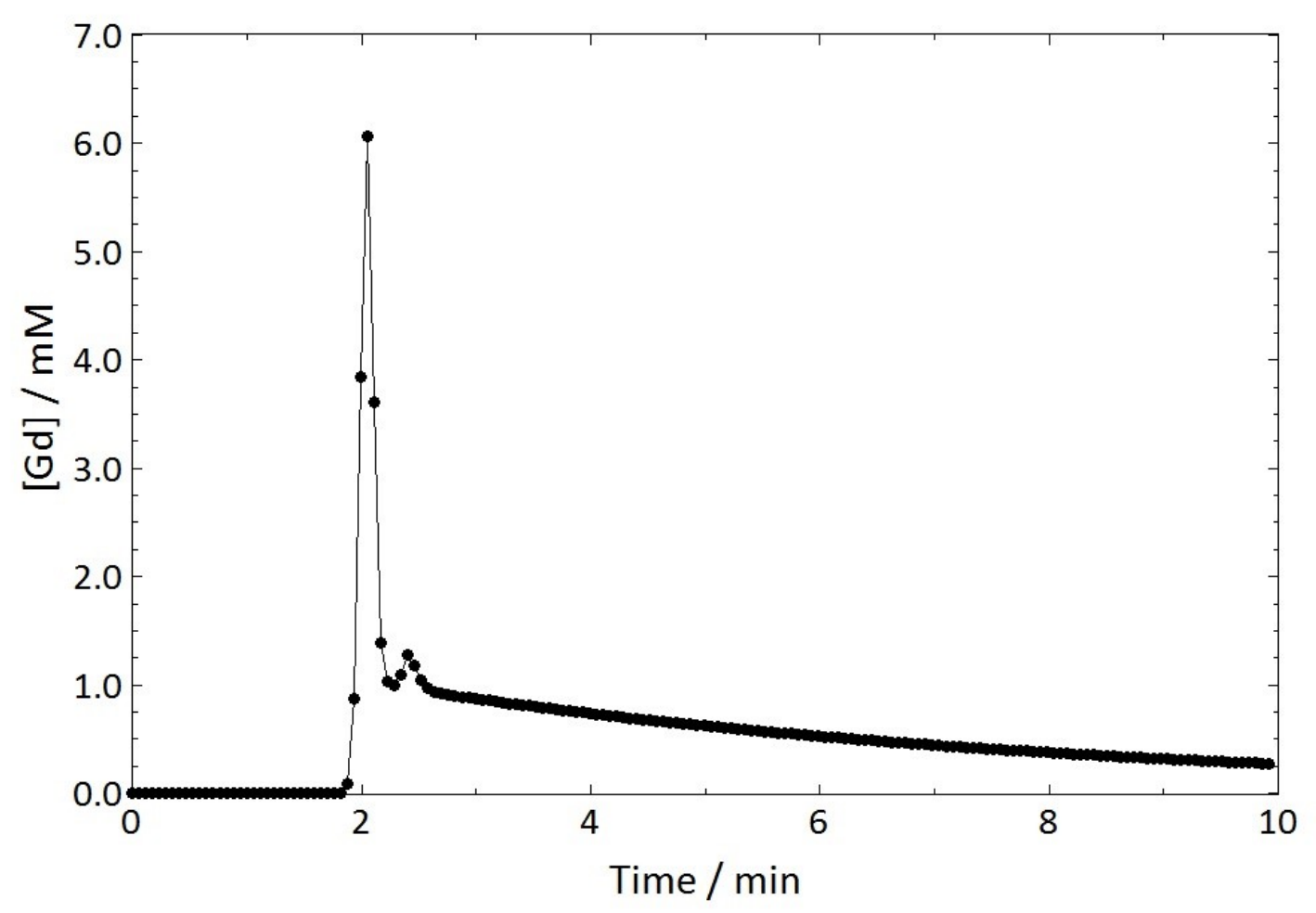

Figure 4.2: AIF created using Eq. 4.13 and data in Table 4.1. The curve was shifted in time to place the peak AIF value at a time that resembles a clinical acquisition. The temporal resolution was changed from $4.97 \mathrm{~s}$ in the original work to $3.5 \mathrm{~s}$ to match the clinical temporal resolution of the DCE-MRI acquisition used in this work. 


\subsubsection{Signal Intensity and Phase AIF Equations}

The AIF as a function of time can be determined by observing either the change in signal intensity (SI) or $\phi$ following GBCA injection. For a GRE sequence, the MR signal intensity $\left(\mathrm{SI}_{\mathrm{GRE}}\right)$ equation is given by Eq. 2.66. Here $\mathrm{T}_{1}$ and $\mathrm{T}_{2}^{*}$ change due to the presence of the GBCA (based on Eqs. 2.84 and 2.85, respectively). However, since the chosen $T_{E}$ is typically much smaller than $T_{2}^{*}$, its effects can be ignored in a GRE sequence. Solving Eq. 2.66 for $[G d](t)$ (i.e. the AIF) based on the change of $\mathrm{SI}$ is given in Eq. 2.100. The change in MR phase $\left(\Delta \phi(\mathrm{t}) \equiv \Delta \phi=\phi(\mathrm{t})-\phi_{0}\right)$ can also be used to compute [Gd](t), as determined in Eq. 2.109. $[\mathrm{Gd}](\mathrm{t})$ curves were computed using both the SI and the $\phi$ approach. The parameters that were used to achieve these calculations are shown in Table 4.2.

With the Parker AIF and SI and $\phi$ equations derived, the following algorithm was performed: (1) use AIF as an input AIF to the simulations; (2) calculate the real and imaginary components for $\mathrm{S}_{\mathrm{GRE}}(\mathrm{t})$; (3) add noise to the real and imaginary functions; (4) recalculate $\mathrm{SI}_{\mathrm{GRE}}(\mathrm{t})$ and $\phi(\mathrm{t})$ functions; (5) introduce additional experimental error; and (6) solve for the output AIF, AIF . These steps are described in greater detail in the sections below.

\subsubsection{Simulated Noise}

The simulation software was created using code written in-house with MATLAB 2017b (The MathWorks, Inc., Natick, MA). The first step for the simulations was to run the program to calculate an AIF from the phase with no simulated noise or other sources of 
Table 4.2: Parameters used to compute the SI- and $\phi$-derived AIFs from the known AlF $_{\mathrm{P}}$ values. $M_{0}$ was chosen arbitrarily, while the values for $\alpha, T_{R}, T_{E}$, and $B_{0}$ were chosen based on the clinical DCE-MRI acquisition performed later in this work.

\begin{tabular}{cc}
\hline Parameter & Value \\
\hline $\mathrm{M}_{0}$ & 1000 \\
$\alpha$ & $30^{\circ}$ \\
$\mathrm{T}_{\mathrm{R}}$ & $6.5 \mathrm{~ms}$ \\
$\mathrm{~T}_{\mathrm{E}}$ & $3.85 \mathrm{~ms}$ \\
$\mathrm{~T}_{1,0}$ & $1932 \mathrm{~ms}^{\dagger}$ \\
$\mathrm{r}_{1}$ & $4.1 \times 10^{3} \mathrm{mM}^{-1} \mathrm{~s}^{-1} \ddagger$ \\
$\gamma$ & $2.68 \times 10^{8} \mathrm{rad} \mathrm{s}^{-1} \mathrm{~T}^{-1}$ \\
$\mathrm{~B}_{0}$ & $3.0 \mathrm{~T}^{-1}$ \\
$\chi_{\mathrm{m}}$ & $3.4 \times 10^{-4} \mathrm{M}^{-1}$ \\
$\theta$ & $0^{\circ}$ \\
\hline+- value taken from Stanisz et al. [72]
\end{tabular}

experimental error as well as for a shift of the whole curve by \pm 0.05 rad as a check to ensure that the program worked properly. As a second software validation step, a linearbased error was added to make sure that the simulated AIF responded in a predictable way. For this case, the $\phi$ value at the peak was considered to be exact while the baseline received either $\mathrm{a} \pm 0.10$ rad or \pm 0.50 rad error. $\phi$ values between the peak and baseline had added errors which were linearly proportional to their original $\phi$ values. [Gd] was again calculated to see the effect of these errors.

More realistically, experimental error observed in an MR acquisition is neither uniform nor does it have a linear dependence. If the image signal-to-noise ratio (SNR) is greater than approximately 3:1, the noise present is Gaussian in appearance [73]. The 
SNR of an SI image can generally be defined as

$$
\operatorname{SNR}(\mathrm{t})=\frac{\mathrm{SI}_{\mathrm{GRE}}(\mathrm{t})}{\sigma_{\mathrm{GRE}}},
$$

where $\sigma_{G R E}$ is the standard deviation of the noise. This noise should affect the real and imaginary parts of the signal separately. Random Gaussian noise was added to the complex GRE signal equation (Eq. 2.66) in the form:

$$
\mathrm{S}_{\mathrm{GRE}}(\mathrm{t})=\mathrm{M}_{0} \mathrm{U}(\mathrm{t}) \cdot\left[\cos \Delta \phi(\mathrm{t})+\mathrm{N}_{\mathrm{R}}(\mathrm{t})+\mathrm{i} \cdot\left(\sin \Delta \phi(\mathrm{t})+\mathrm{N}_{\mathrm{l}}(\mathrm{t})\right)\right]
$$

where $N_{R}$ and $N_{1}$ are the noise values independently sampled from a Gaussian distribution with variance $\sigma_{G}^{2}$, applied to the real and imaginary components, respectively; $U(t)$ is the total relaxation component of Eq. 2.66, represented as

$$
\mathrm{U}(\mathrm{t})=\frac{1-\mathrm{e}^{-\mathrm{T}_{\mathrm{R}} / \mathrm{T}_{1}(\mathrm{t})}}{1-\cos \alpha \cdot \mathrm{e}^{-\mathrm{T}_{\mathrm{R}} / \mathrm{T}_{1}(\mathrm{t})}} \sin \alpha,
$$

where relaxation effects due to $T_{2}^{*}$ are ignored (since $\left.T_{E} \ll T_{2}^{*}\right)$ and $\Delta \phi(t)$ is the change in phase from its value at $t=0$ caused by the passage of the bolus of GBCA. The value of $\Delta \phi(t)$ was calculated from Eq. 2.109 with $[G d](t)$ set equal to $A_{I F}(t)$.

The noise terms were designed to be uncorrelated. Based on Eq. 4.15, the Gaussian noise that was effectively added to the real and imaginary terms is multiplied by $M_{0} U(t)$. This results in an overall noise for the $G R E$ data of $\sigma_{G R E}=M_{0} U(t) \sigma_{G}$. The minimum SNR for a DCE-MRI scan corresponds to the time before the bolus of GBCA arrives, so for this situation $\sigma_{\mathrm{GRE}}=\mathrm{M}_{\mathrm{O}} \mathrm{U}(0) \sigma_{\mathrm{G}}$. For the simulations reported here, the $\mathrm{SNR}$ value will be considered to be the SNR of the baseline and is therefore,

$$
\operatorname{SNR}=\operatorname{SNR}(0)=\frac{M_{0} U(0)}{M_{0} U(0) \sigma_{G}}=\frac{1}{\sigma_{G}}
$$

Different $\sigma_{G}$ values were applied to determine the effect that Gaussian noise has on the 
signal and phase equations to determine [Gd]. The chosen SNR values ranged from 3:1 to 100:1.

\subsubsection{Partial Volume Averaging}

A simple computer model was created to simulate the effects of PVA (Eq. 3.30) on the acquisition of the PO and PT AIFs measured in a vessel. The model assumed no Gaussian noise was present and the PO AIF was calculated from the ROI-averaged phase in a vessel.

$\phi^{\text {in }}$ was computed using Eq. $3.50, \Delta \phi_{x}$ was computed using Eq. 3.38 , and the model assumed no flow effects on the measured phase (i.e. $\Delta \phi_{F}=0$ ). The Hct value used was the average for the clinical study presented later in the thesis. All other parameters that were used are provided in Tables 3.2 and 4.2. The PT AIF measurement used the $T_{1}$ values calculated using Eq. 2.84 from the $\mathrm{AlF}_{\mathrm{p}}$ value at the 6-minute mark for convenience.

\subsection{Results}

\subsubsection{Simulated AIFs with Added Noise}

The SI- and $\phi$-derived (i.e. the PO) simulated AlFs were first computed from the input $\mathrm{AlF}_{\mathrm{P}}$ function using Eqs. 2.100 and 2.109, respectively. These two curves, along with $\mathrm{AlF}_{\mathrm{p}}$, are plotted in Fig. 4.3. As expected, the three curves are identical over the entire range of the AIF. Different types of error were then applied to the model. First, a constant $\pm 0.05 \mathrm{rad}$ $\phi$-error was applied to the whole $\mathrm{AlF}_{\mathrm{p}}$. An example of this, and subsequent $A \mathrm{AF}_{\mathrm{O}}$ (i.e. output AIF calculation) are shown in Fig. 4.4. Due to the subtraction of the $\operatorname{AlF}_{\mathrm{p}} \phi_{0}$ values 


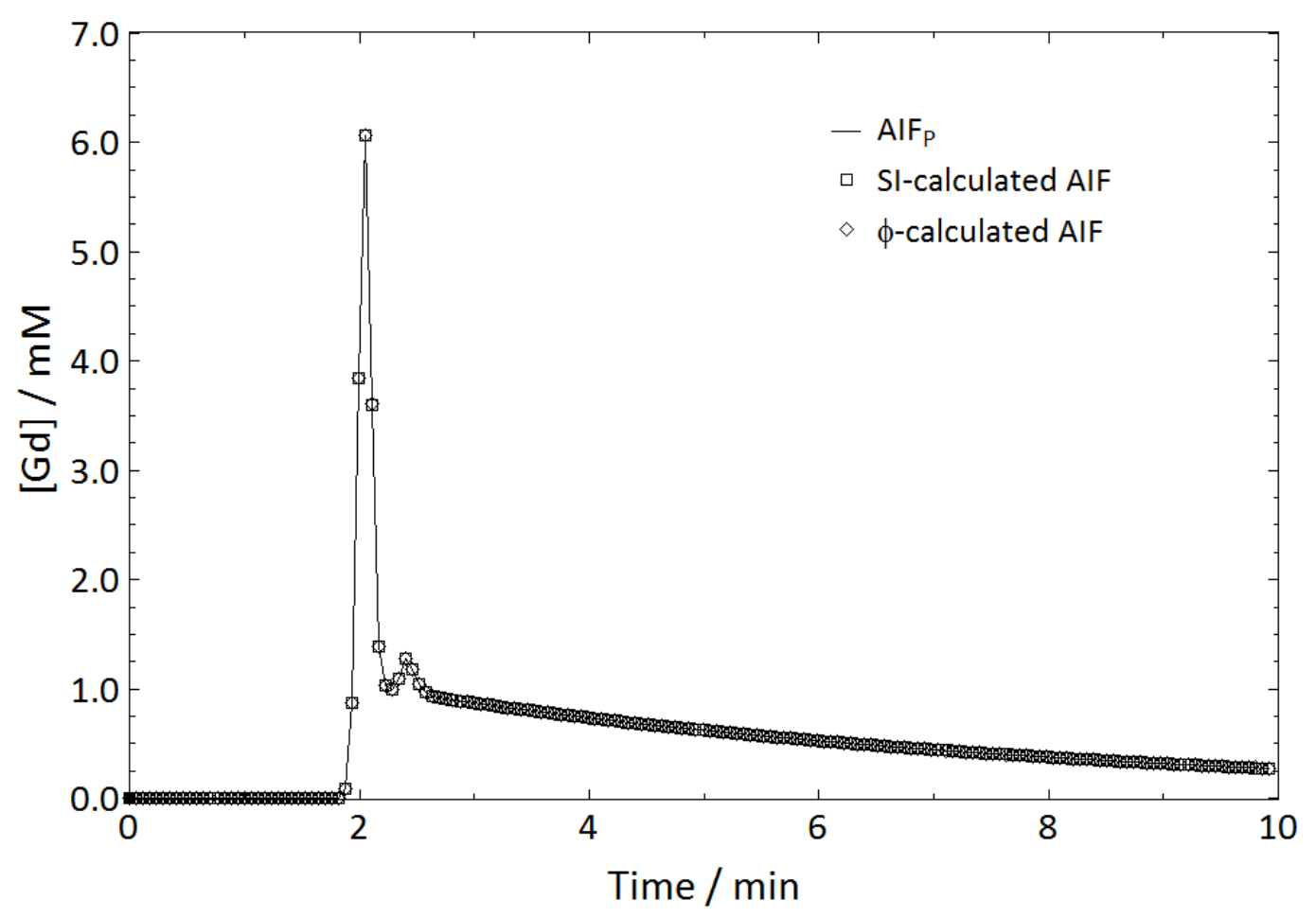

Figure 4.3: AIF curves derived from SI-calculated (squares) and $\phi$-calculated (diamonds) AIFs computed from the original AIF $F_{p}$ curve (line). The three data sets have identical AIF values over the entire range.

(Eq. 4.2), a uniform error does not affect the computation of AlF $F_{p}$. A linearly scaled error ( $\phi$-error $=0$ at $\mathrm{AlF}_{\mathrm{P}}$ peak, $\phi$-error $= \pm 0.10$ or \pm 0.50 rad at $\mathrm{AIF}_{\mathrm{P}}$ baseline) was applied and the $\mathrm{AlF}_{\mathrm{O}}$ was again calculated, as shown in Figs. 4.5 and 4.6. From Fig. 4.5A and 4.6A, the peak phases all are unaffected, but the baseline $\phi$ values differ by that input error ( 0.10 or $0.50 \mathrm{rad}$, respectively). Once the corresponding AIF $\mathrm{O}_{\mathrm{O}}$ curves were calculated, all baseline values matched $(0 \mathrm{mM})$, but in each instance the rest of the curves were different (Figs. 4.5B and 4.6B). The uncertainty of the AIF values increases with [Gd] and is quite large at the peak.

Various amounts of Gaussian noise were added to the SI and $\phi$ data of AlF $_{\mathrm{P}}$, as 

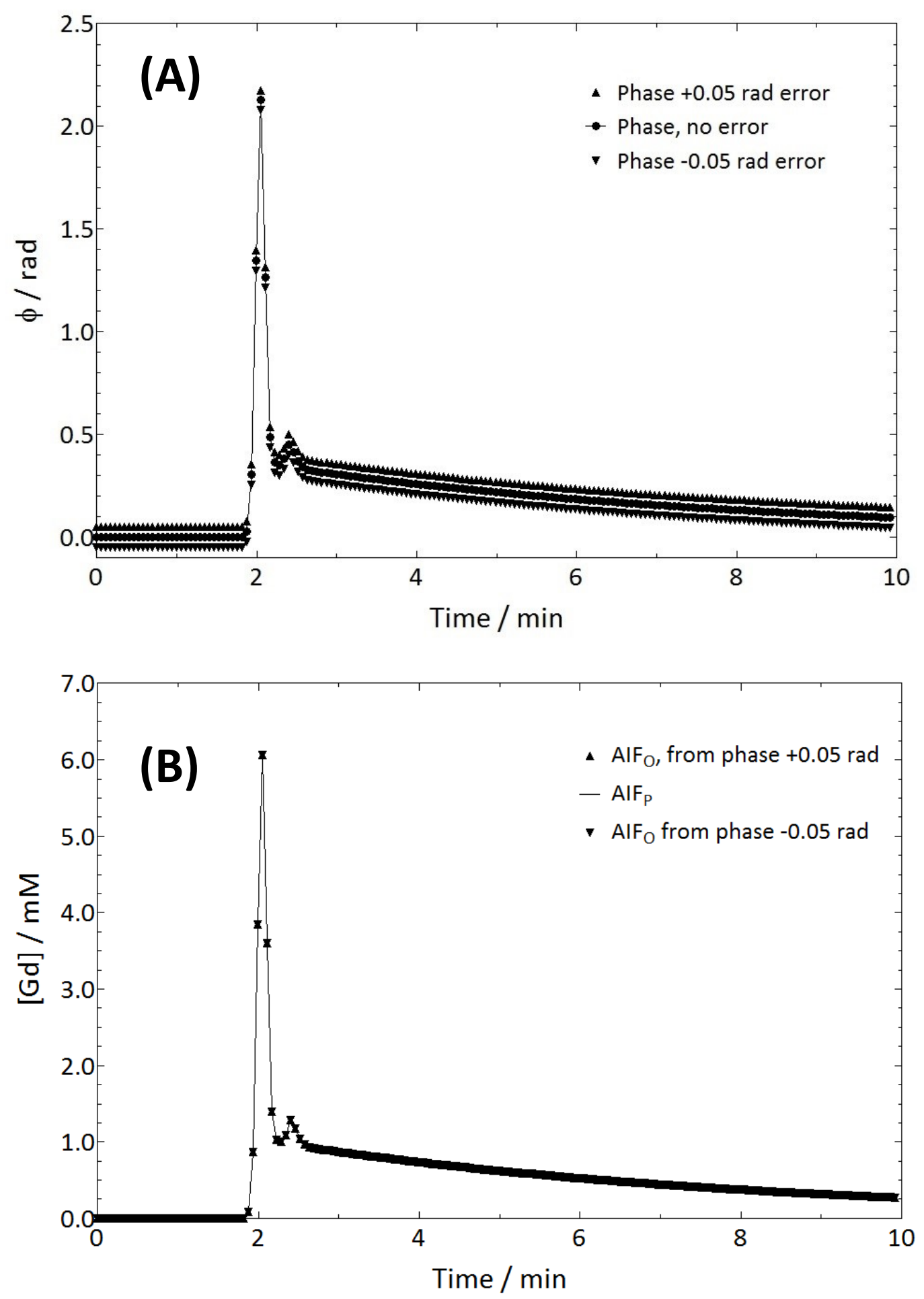

Figure 4.4: (A) The measured phase values from AIF (circles) along with a +0.05 rad (upward triangle) and - 0.05 rad (downward triangle) constant error. (B) The calculated $\mathrm{AlF}_{\mathrm{O}}$ values for each of the three curves in (A). Note that the use of Eq. 4.2 removes any baseline error values from the rest of the AIF $_{O}$ curve resulting in uniform AIF values in (B). 

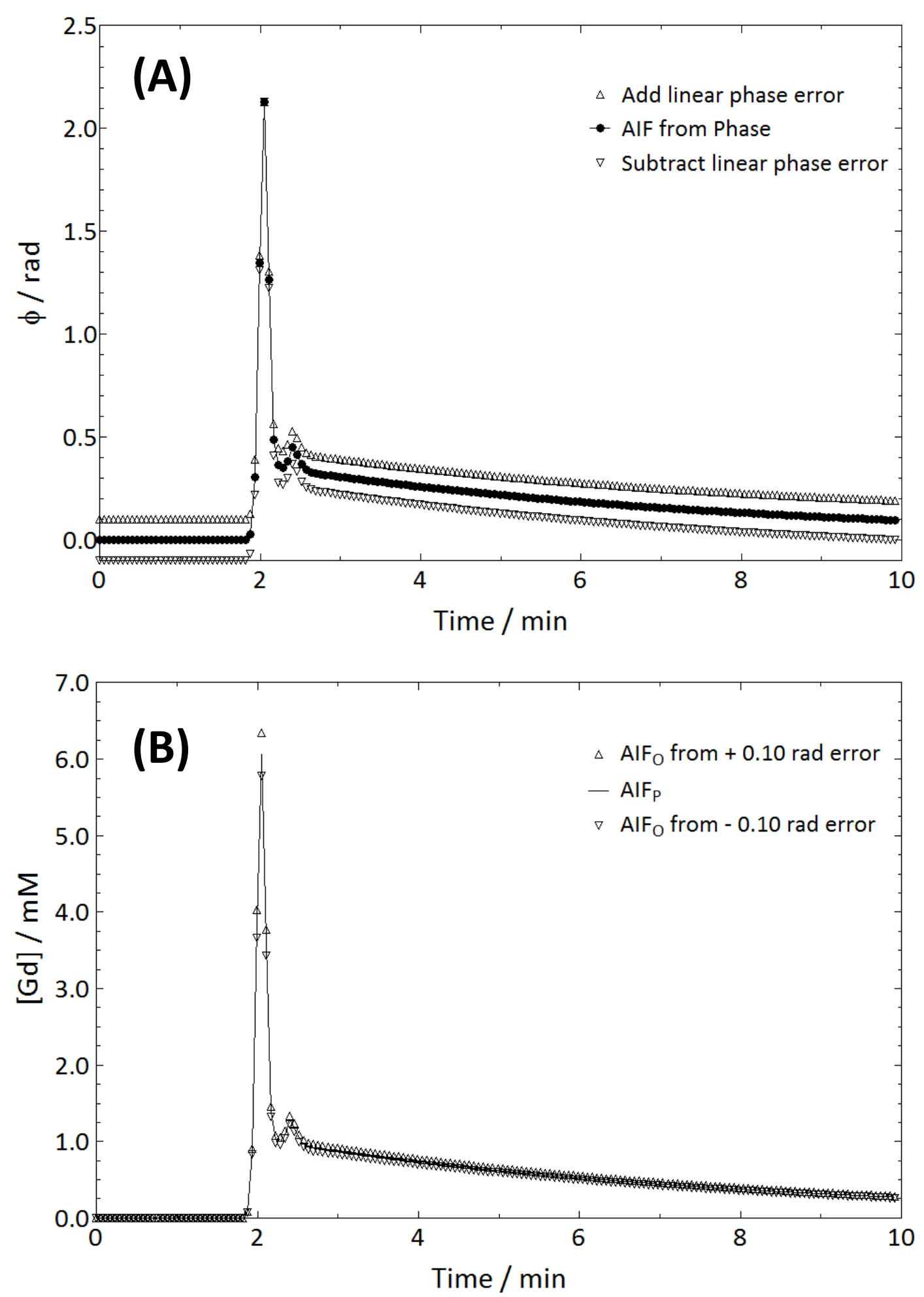

Figure 4.5: (A) The $\phi$ values from AlF $_{\mathrm{p}}$ (circles) along with a linearly-proportional error such that AIF $\phi$-error $=0$ at peak and was +0.10 rad (upward triangle) and -0.10 rad (downward triangle). (B) The calculated AIF values for each of the three curves in (A). 

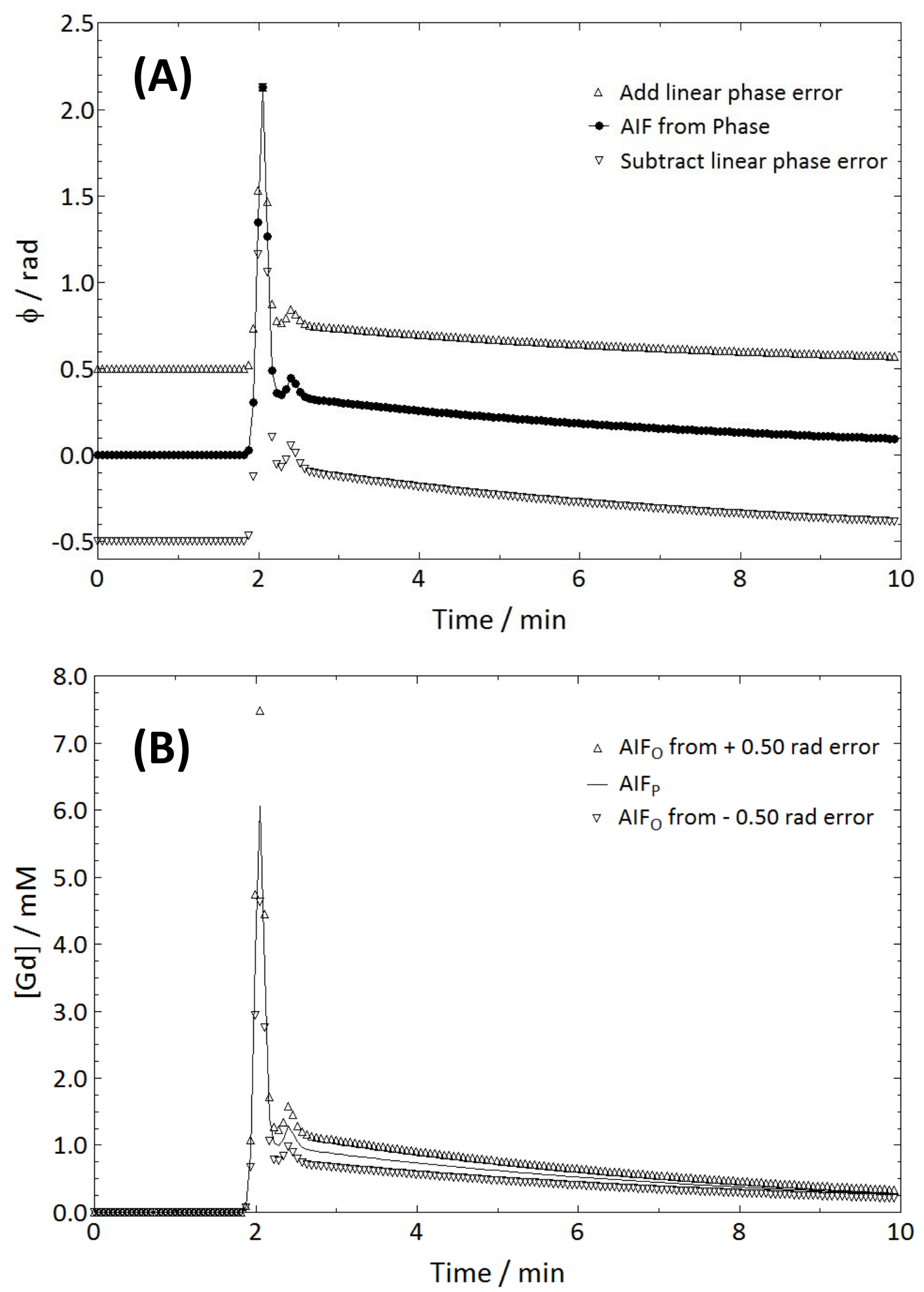

Figure 4.6: (A) The $\phi$ values from AlF $_{\mathrm{p}}$ (circles) along with a linearly-proportional error such that AIF $\phi$-error $=0$ at peak and was +0.50 rad (upward triangle) and -0.50 rad (downward triangle). (B) The calculated AIF values for each of the three curves in (A). 
given by Eq. 4.15, to generate SNR data that ranged from 3:1 to 100:1 using Eq. 4.17. AIF data computed with this added Gaussian noise are shown in Figs. 4.7-4.9 (using SI data) and 4.10-4.12 (using $\phi$ data). The results shown in the figures are the average of five simulations. Although the fluctuations of the simulated PO AIF values about AIF decrease with increasing SNR, no overall shifts or distortions were seen. From the results of the SI simulations, it was observed that the uncertainty of the AIF values increases as [Gd] increases and is much higher at the peak than it is for the rest of the AIF.

\subsubsection{The Effects of Partial Volume Averaging}

In Fig. 4.13, a PO AIF is shown with phase error due to PVA, $\Delta \phi_{\mathrm{PV}}$, where $\Delta \phi_{\mathrm{PV}}$ was calculated using the equations derived in Chapter 3. It can be seen from the figure that the PO AIF differs significantly from the true AIF which, in this case, is AIF . Using the new method for calculating the AIF introduced in Section 4.2, the corresponding PT AIF was also simulated. This PT AIF (see Fig. 4.13) is in much better agreement with AlF $_{\mathrm{P}}$ than the PO AIF is for every point of the AIF other than the baseline.

\subsection{Discussion}

Traditionally, the AIF can be computed using either the SI or $\phi$. In many clinical settings, a pseudo-AIF which is simply generated directly from SI changes is used for DCE-MRI analysis. When this approach is used, the parameters calculated are considered 'semiquantitative'. Quantitative DCE-MRI parameters can be obtained, however, when they are calculated using a true AIF measured in terms of [Gd]. The reason why SI is typically 

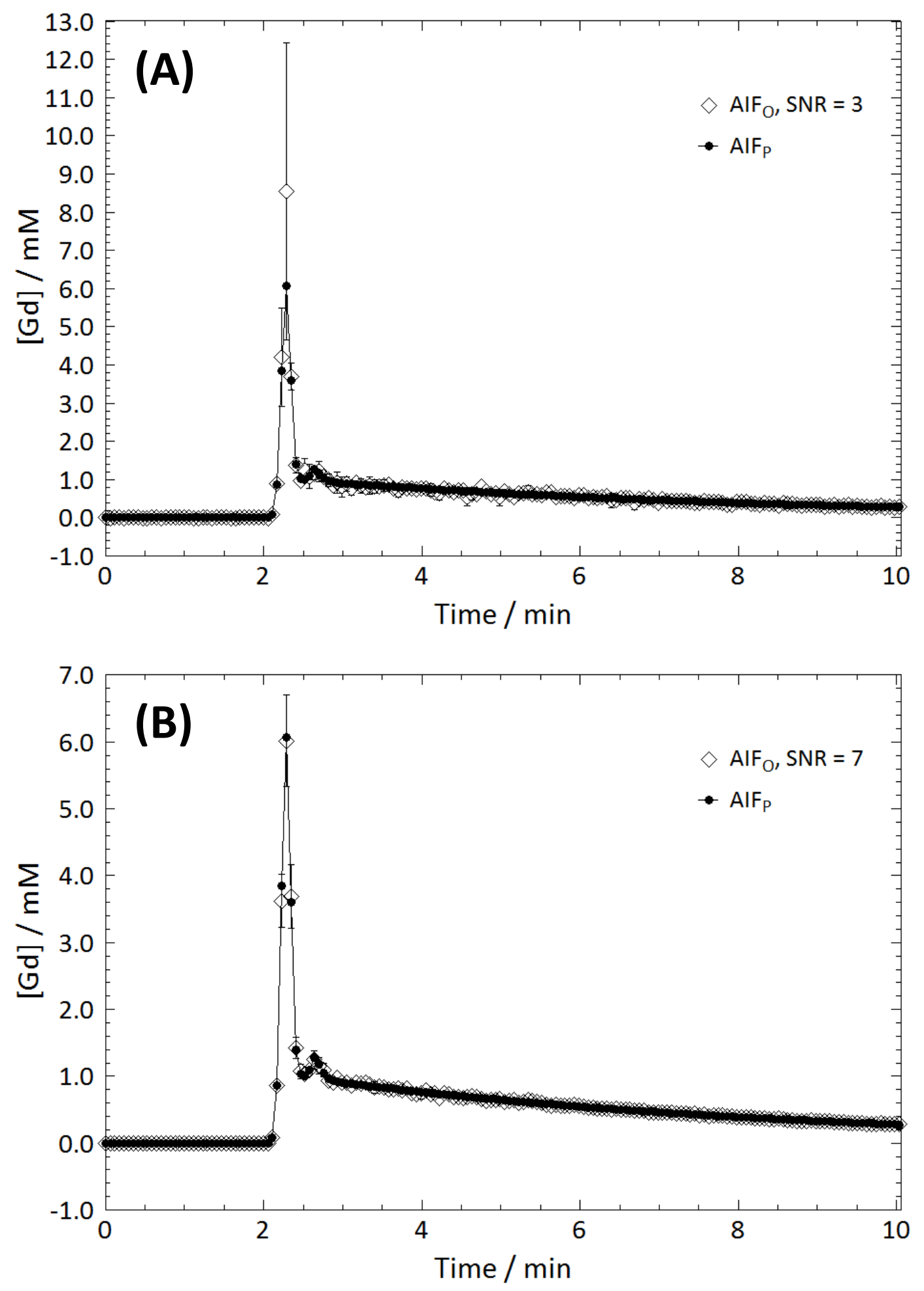

Figure 4.7: AlF $_{\mathrm{O}}$ computation using $\mathrm{SI}$ based on the average of five simulations with defined input SNR values of (A) 3 and (B) 7. Error bars represent the standard deviation of the five simulation results. 

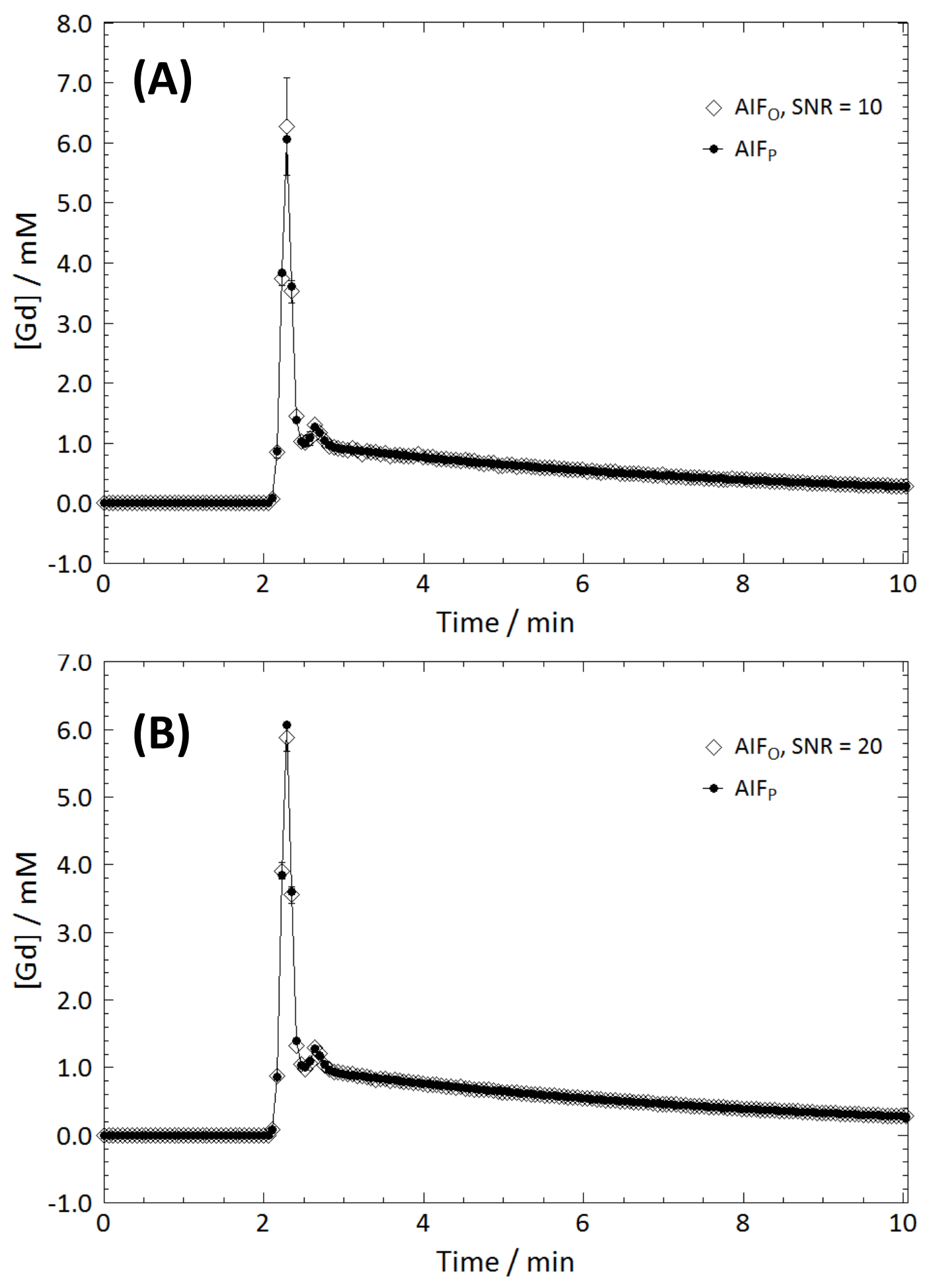

Figure 4.8: $\mathrm{AlF}_{\mathrm{O}}$ computation using $\mathrm{SI}$ based on the average of five simulations with defined input SNR values of (A) 10 and (B) 20. Error bars represent the standard deviation of the five simulation results. 

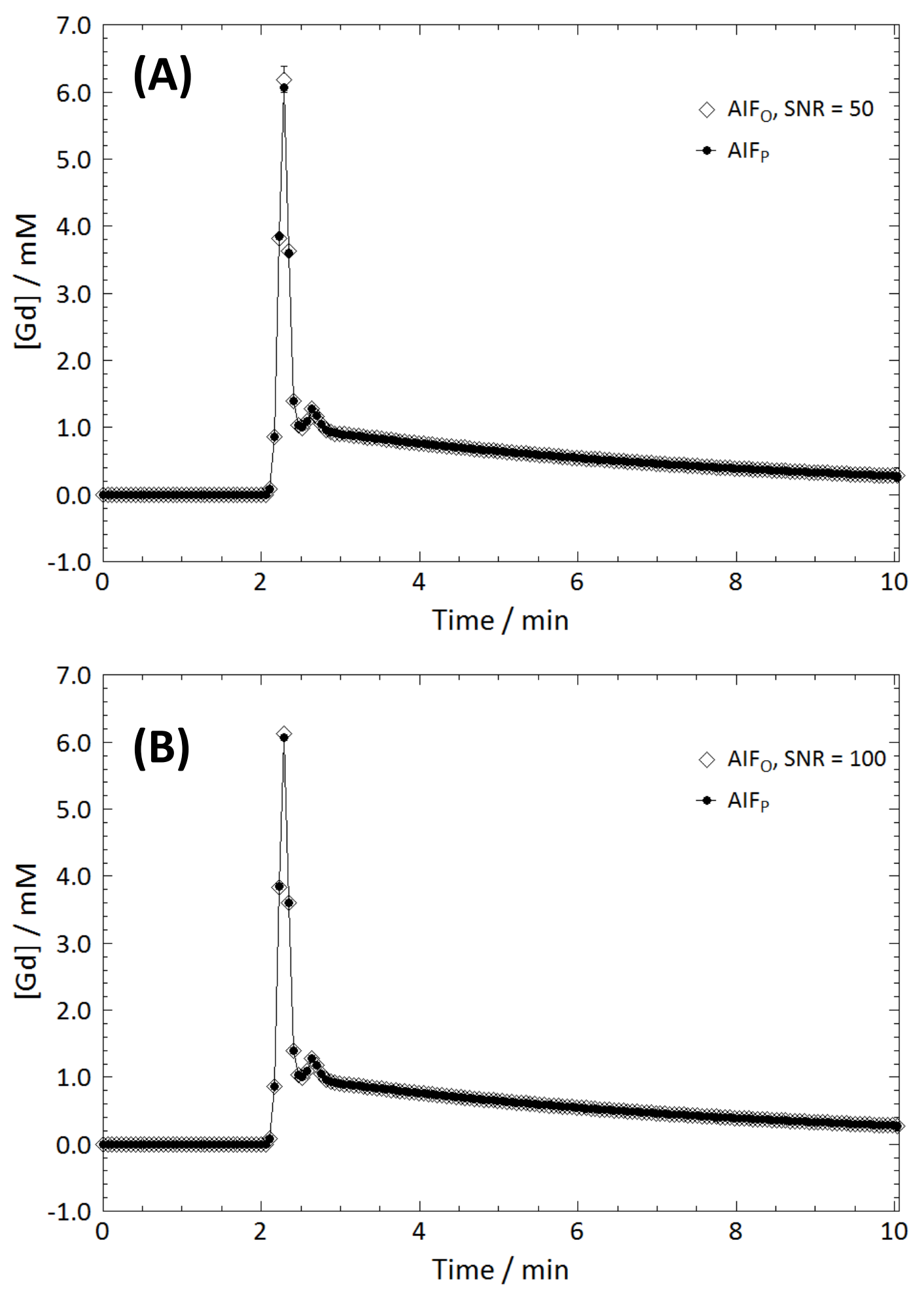

Figure 4.9: $\mathrm{AIF}_{\mathrm{O}}$ computation using $\mathrm{SI}$ based on the average of five simulations with defined input SNR values of (A) 50 and (B) 100. Error bars represent the standard deviation of the five simulation results. 

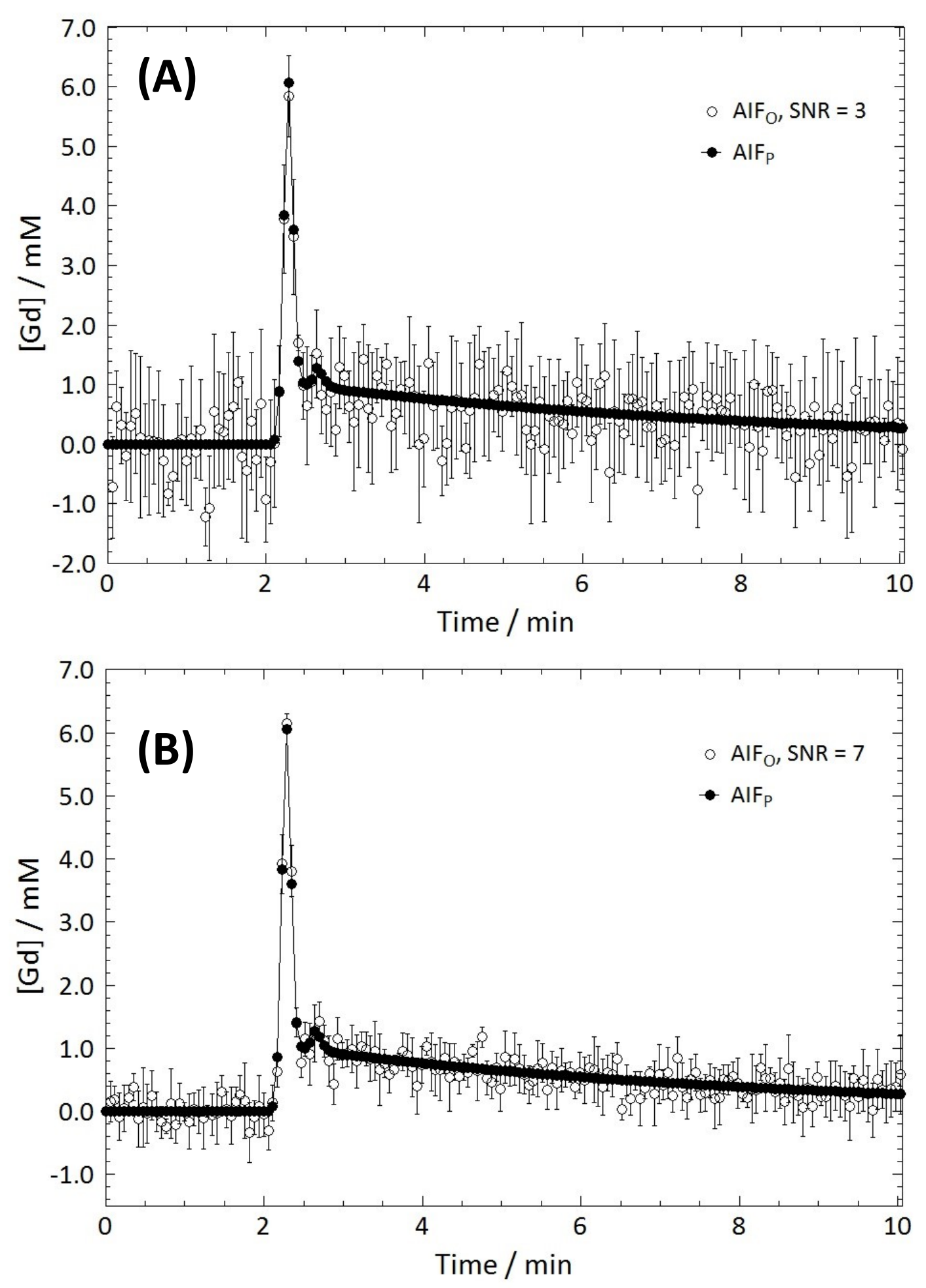

Figure 4.10: AlF $_{\mathrm{O}}$ computation using $\Delta \phi$ based on the average of five simulations with defined input SNR values of (A) 3 and (B) 7. Error bars represent the standard deviation of the five simulation results. 

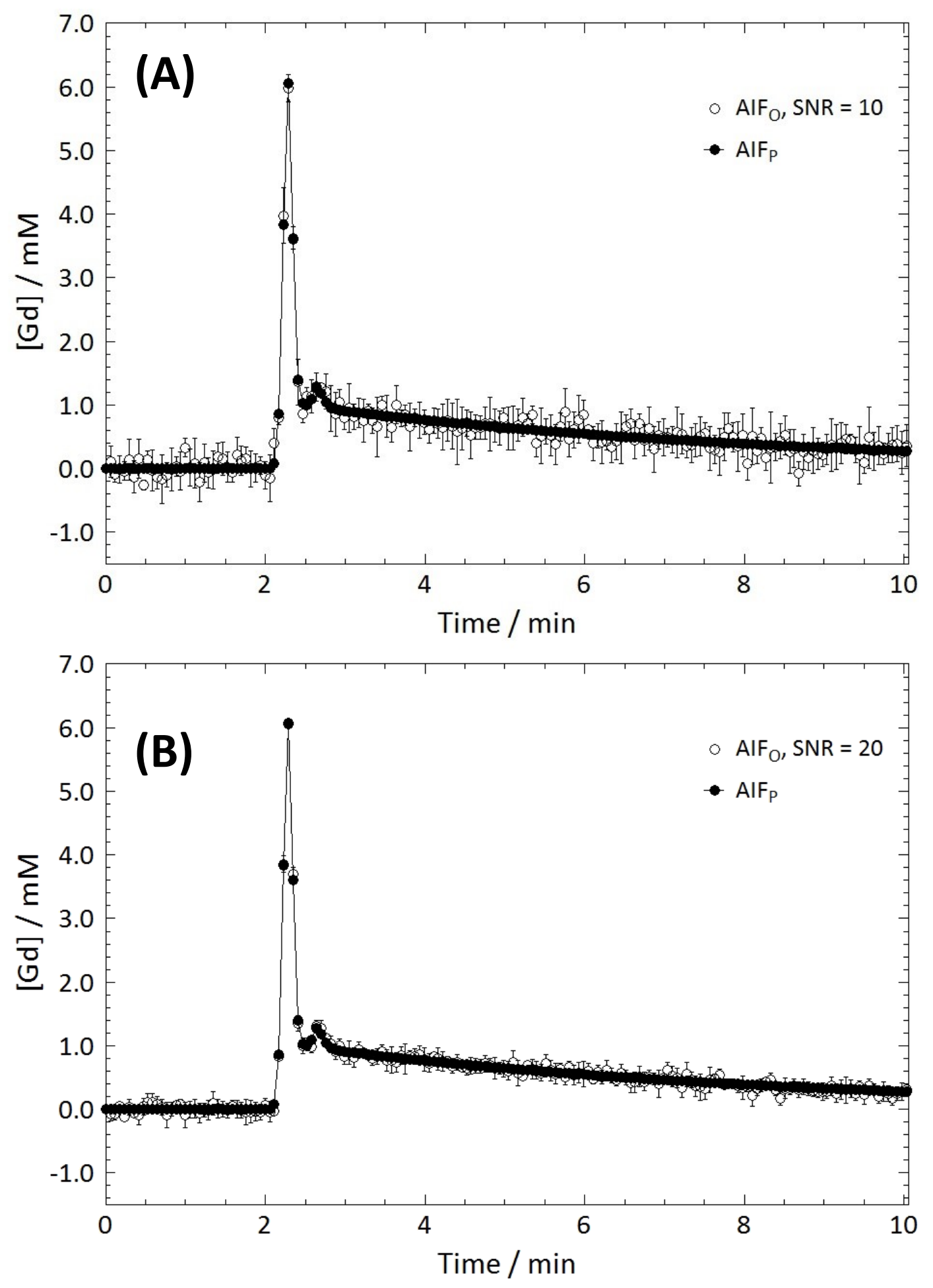

Figure 4.11: AlF $_{\mathrm{O}}$ computation using $\Delta \phi$ based on the average of five simulations with defined input SNR values of (A) 10 and (B) 20. Error bars represent the standard deviation of the five simulation results. 

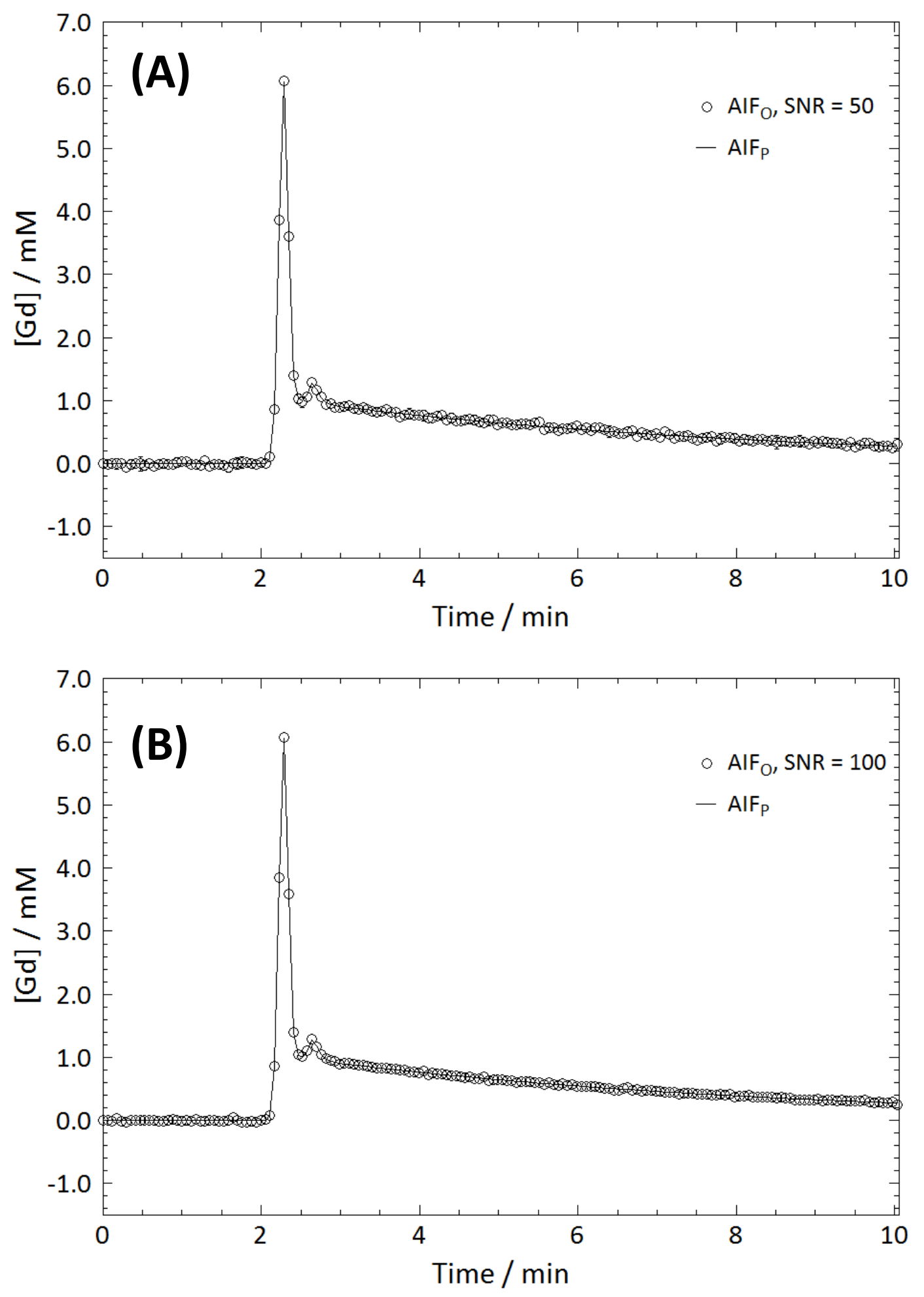

Figure 4.12: AlF $F_{O}$ computation using $\Delta \phi$ based on the average of five simulations with defined input SNR values of (A) 50 and (B) 100. Error bars represent the standard deviation of the five simulation results. 


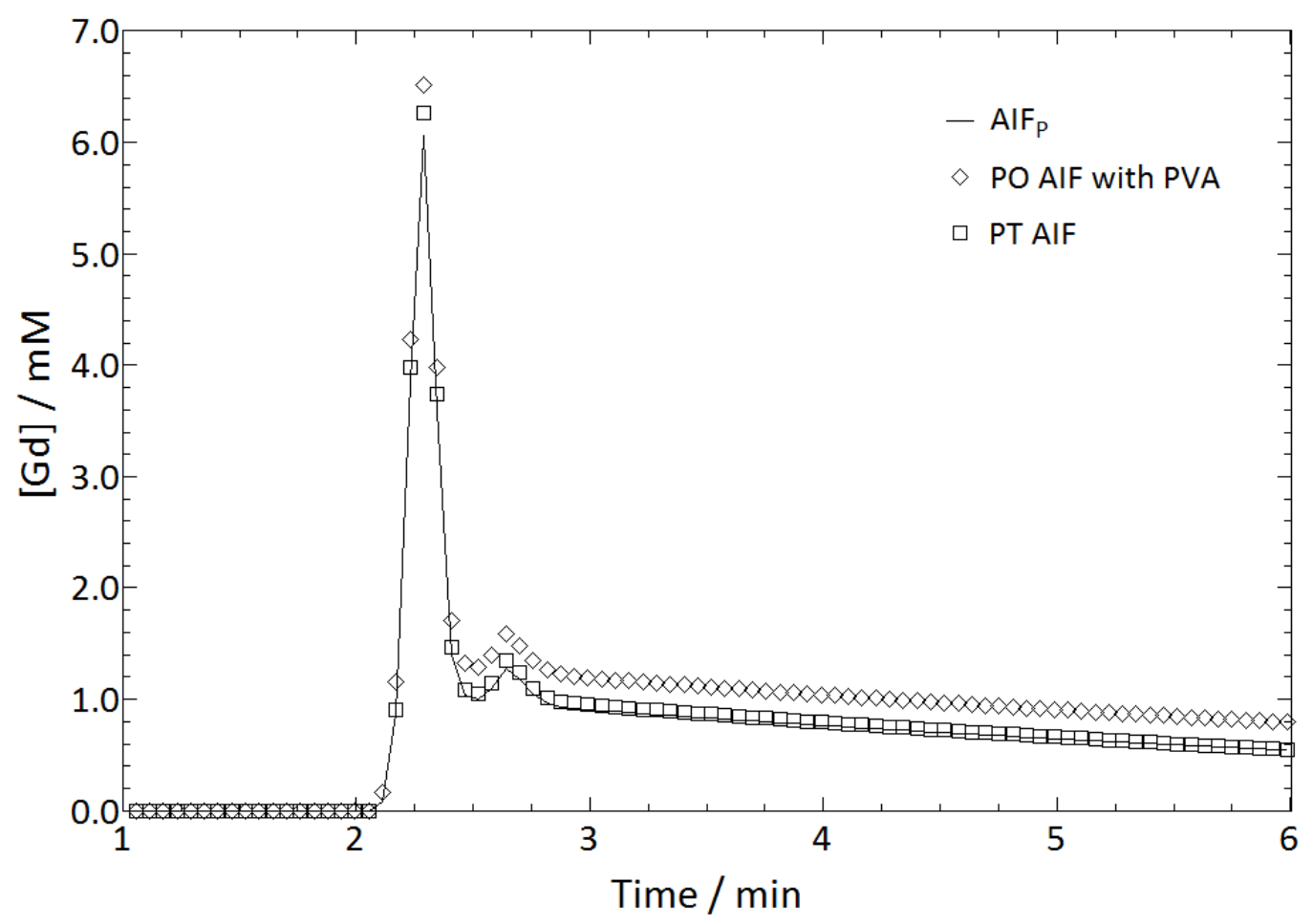

Figure 4.13: The effects of PVA on the measurement of the PO AIF in comparison with the PT AIF to the true input, AIF . The curve was limited to this time-frame (1-6 min) to better observe the difference in acquisition values at the AIF peak.

chosen is because it is readily available with each MR acquisition and is obtained without the need for any post-processing. While MR $\phi$ data are computed for every sequence, they are not always displayed nor is it obvious how to extract the data from the MR scanner. There are several reasons why using either the SI method or the $\phi$ method alone can result in inaccurate AIF measurements. One of the primary issues using SI only is the insensitivity to changes in [Gd] (i.e. it saturates) for the moderately high [Gd] values that occur in blood during the first-pass of the GBCA bolus [76]. Computing the AIF using the phase of the MR signal avoids this problem. Additionally, $\Delta \phi$ is linear with respect to changing [Gd] as seen in Eq. 2.109. 
It was shown in Chapter 3 that the $\phi$ measurements used to compute the AlF are prone to a systematic experimental error as a result of PVA where this phase error, $\Delta \phi_{\mathrm{PV}}$, is additive and decreases as [Gd] increases. In particular, $\Delta \phi_{\mathrm{PV}}$ was shown to be considerably higher for the baseline than it is for the washout. This is unfortunate since, with the method conventionally used to calculate the AIF from $\phi$ measurements (the PO AlF method), the baseline phase (along with its relatively large $\Delta \phi_{\mathrm{PV}}$ ) is subtracted from each data point to get the final AIF. The baseline phase error, therefore, becomes a large part of the experimental error for each point of the AIF.

In Section 4.2, a new method for calculating the AIF from $\phi$ measurements (the PT AIF method) was proposed. With this approach the AIF is determined from $\phi$ changes relative to $\phi_{w^{\prime}}$, the phase measured for the AIF washout rather than $\phi_{0}$. An error analysis also presented in Section 4.2 shows that the PT AIF method can be expected to be a considerable improvement over the PO AIF method. An important assumption for this method is that the true $[G d]$ value for the AIF washout is available. It is expected that this accurate washout can be provided by $T_{1}$ measurements. This topic is covered in the next Chapter. It should be noted that it doesn't really matter where this value comes from as long as a reliable value is available from some source.

The effect of noise and experimental error on the AIF was further investigated using computer simulations. Software was written to simulate the computation of an AIF from measurements of either the MR SI or the $\phi$ with several different sources of experimental error incorporated into the code. These included Gaussian random noise, a constant offset, and phase error that was inversely proportional to [Gd]. The results of 
these simulations demonstrate that there is no discernable shift or distortion of the PO AIF due to Gaussian random noise. Fluctuations of the data points about the true AIF increase as SNR decreases but the shape of the function is not affected.

From the results of the simulations where SI was used to calculate the AIF, it can be seen by looking at the error bars that the uncertainty for the AIF values increases sharply as $[\mathrm{Gd}]$ increases. This can be seen very clearly in Fig. 4.7A where the uncertainty at the peak is quite large. This is a manifestation of the saturation effect where a small error in the SI value leads to a larger [Gd] uncertainty as [Gd] increases.

The effect of a phase error that decreases linearly with [Gd] was also investigated. There is no reason to believe that this dependence is strictly linear in reality; however, the analysis presented in Chapter 3 indicates that, even if it is not linear, the behaviour of $\Delta \phi_{\mathrm{PV}}$ can be expected to be similar insofar as it decreases monotonically with increasing [Gd]. These linear phase error simulations showed quite clearly (see Figs 4.5 and 4.6) that a big baseline error leads to a large uncertainty for the peak of the AIF. This general behaviour can be expected for monotonically decreasing phase error with increasing [Gd] even if the dependence is not linear.

The most important simulation results presented in this chapter are given in Section 4.4.2 where the PT AIF is shown to be significantly superior to the PO AIF (see Fig. 4.13). The correction applied in going from the PO AIF to the PT AIF is not perfect; if it

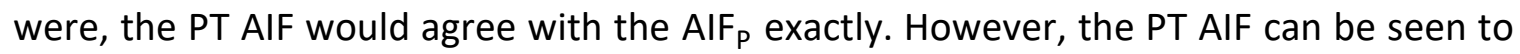
be considerably more accurate than the PO AIF at all points along the curve (other than the baseline) including at the peak. 


\subsection{Conclusions}

The contribution of PVA to MR phase measurements used to compute a PO AIF was investigated in Chapter 3 where it was found that PVA causes an additive phase error which is inversely proportional to [Gd]. This was extended in this chapter to a consideration of other forms of experimental error using simulations. The work of the previous chapter lead to the development of the PT AIF method, a novel approach for computing the AIF from phase measurements. An error analysis presented in Section 4.2 predicts that the PT AIF method should be considerably more accurate than the PO AIF method. This was confirmed using computer simulations of both a PO AIF and the PT AIF generated from a model AIF (e.g. the Parker AIF).

The main drawback of the PT AIF method is that an accurate value for [Gd] during the AIF washout phase is required. It is proposed that $T_{1}$ measurements can provide this information. This will be investigated in the following two chapters. 


\section{Chapter 5}

\section{AIF Experiments Using Phantoms}

\subsection{Introduction}

In the previous two chapters the need for a more accurate method to obtain the arterial input function (AIF) in clinical DCE-MRI acquisitions was introduced. The most commonly utilized AIF computation method uses MR signal intensity (SI); however, the use of MR phase $(\phi)$ has become more popular for DCE-MRI in recent years $[50,58,77,78]$, although its use clinically is still very limited. Two recent works have incorporated both SI and $\phi$ into the computation of the AIF to utilize the advantages of each technique $[79,80]$, but this method has not seen much use clinically, especially in the brain. As described in Section 3.2.1, the major benefit of using $\phi$ to compute the AIF, is that the change of $\phi$ is linear with respect to the changing GBCA concentration ([Gd]). However, an AIF obtained with $\phi$ only (i.e. the PO AIF) has its own flaws. It was shown in Chapters 3 and 4 that the phase error can be expected to increase as [Gd] decreases with the maximum phase error 
occurring when $[\mathrm{Gd}]=0$. To calculate the $[\mathrm{Gd}]$ values using the conventional approach for evaluating the AIF from phase data, the phase for the AIF baseline (i.e. when $[\mathrm{Gd}]=$ $0)$ is subtracted from all the other $\phi(t)$ values. Even though the other $\phi(t)$ values used for the AIF calculation are inherently more accurate (since $[G d]>0$ ), the overall accuracy of the PO AIF is determined primarily by the uncertainty of the baseline phase.

In Chapter 4 a better approach was proposed. With this new technique, called the PT AIF method, the AIF is calculated from phase changes relative to the AIF washout (i.e. the end of the AIF curve when [Gd] has reached an equilibrium value greater than zero) phase rather than the troublesome baseline phase. The PT AIF method also requires the measurement of $T_{1}$ for both the baseline and the washout. Unfortunately, $T_{1}$ measurements are normally too time consuming to be feasible for inclusion in a clinical protocol. One of the most time-efficient methods to measure $T_{1}$ is the technique proposed by Look and Locker [33]. However, this technique was not available on commercial scanners until recently when a modified version known as MOLLI was introduced for measuring cardiac $T_{1}$ 's. The work reported in this thesis uses a MOLLI sequence that was adapted for brain imaging.

In this chapter, the PT AIF method using MOLLI $\mathrm{T}_{1}$ measurements is validated using two types of phantoms (i.e. test objects). The first set of phantoms held static GBCAdoped water at various [Gd] levels that covered the range of values anticipated for the AlF baseline (i.e. $[\mathrm{Gd}]=0$ ) and washout (i.e. $0<[G d]<1 \mathrm{mM}$ ). $\mathrm{T}_{1}$ was measured for each phantom. These data were used to create a calibration curve which allows for the determination of $[G d]$ for any sample by measuring its $T_{1}$ value. The second phantom type 
used a flowing-water, or dynamic, plastic tubing system that was used to obtain a PO AIF similar to the ones measured in vivo. Using the method established for static phantoms, $\mathrm{T}_{1}$ was computed in the baseline and washout regions of the AIF measured for this dynamic phantom system. These $T_{1}$ measurements were used in conjunction with the PO AlF to construct the more accurate PT AIF for the phantom. The first set of phantoms with non-flowing GBCA-doped water will be referred to in the thesis as static phantoms while the phantom with flowing water will be called the dynamic phantom.

\subsection{Methods}

All phantom data were acquired on a Siemens 3 T Magnetom Trio (Siemens AG, Erlangen, Germany) with a 32-channel head coil. For all experiments, the GBCA used was gadobutrol (Gadovist ${ }^{\circledR}$ 1.0 M from Bayer HealthCare Pharmaceuticals, Toronto, Canada).

\subsubsection{Phantom Design and Creation}

\subsubsection{Static Phantoms}

Each static phantom was filled with a specific amount of GBCA and water to establish certain [Gd] values. The goal was to create phantoms with [Gd] values that spanned the range of [Gd] that was expected to occur for the washout during a clinical AIF acquisition. The washout [Gd] values, based on the population-averaged AIF given by Parker et al. [67] and shown in Fig. 4.2, can be expected to be between 0 and $1.5 \mathrm{mM}$. The corresponding $\mathrm{T}_{1}$ values can be calculated from Eq. 2.84 (with $\mathrm{r}_{1}=4.1 \times 10^{3} \mathrm{mM}^{-1} \mathrm{~s}^{-1}[43]$ and $\mathrm{T}_{1,0}=$ 
$1932 \mathrm{~ms}$ [72]) to be between about 150 and $2000 \mathrm{~ms}$.

A series of 14 thin-walled, sealed, straight plastic tubes was created. The phantoms had an inner diameter of $10.5 \pm 0.5 \mathrm{~mm}$ and a volume of approximately $20 \mathrm{~mL}$. The phantom design was chosen to resemble the mid-occipital region of the superior sagittal sinus (SSS) in human adults, which has a diameter of $9.9 \pm 2.4 \mathrm{~mm}$ [57]. One of the static phantoms used in these experiments is shown in Fig. 5.1. To construct these phantoms, a small quantity of the GBCA was drawn from a vial using a small-volume syringe ( $1 \mathrm{~mL}$ volume, $0.01 \mathrm{~mL}$ precision) and added to a larger volume of roomtemperature tap water ( $1 \mathrm{~L}$ volume, $20 \mathrm{~mL}$ precision) to create the desired [Gd]. The solution was thoroughly mixed to ensure a uniform [Gd] throughout the container. The solution was then placed in the tube phantom and sealed on both ends using wax. This process was repeated with different quantities of GBCA and water to create the remaining phantoms.

The volumes of GBCA, water, and their resulting [Gd] values for each of the static phantoms are shown in Table 5.1. Volume measurement errors for each container were taken to be the same as the container precision to establish a conservative, upper limit on the measurement errors. The resulting [Gd] error was computed using the standard error propagation equation [81]:

$$
\sigma_{[G d]}=\sqrt{\left(\frac{\partial[G d]}{\partial V_{G B C A}} \sigma_{V_{G B C A}}\right)^{2}+\left(\frac{\partial[G d]}{\partial V_{w}} \sigma_{V_{w}}\right)^{2}},
$$

which reduces to 


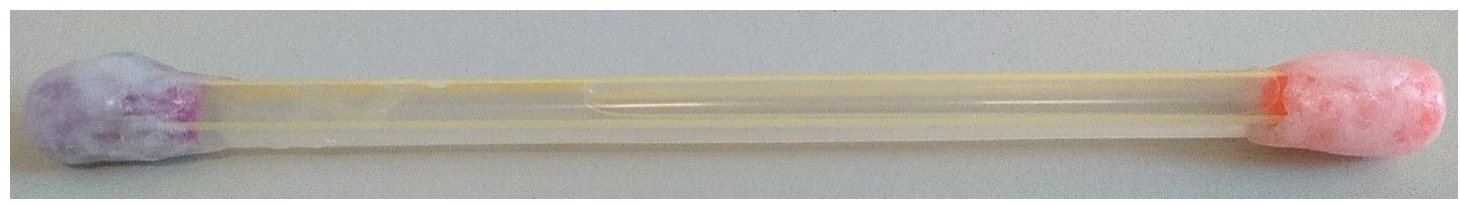

Figure 5.1: An example of the tube phantoms used to measure $T_{1}$.

Table 5.1: GBCA volume $\left(\mathrm{V}_{\mathrm{GBCA}}\right)$, water volume $\left(\mathrm{V}_{\mathrm{w}}\right)$, and their respective [Gd] with uncertainty for the 14 static phantoms.

\begin{tabular}{ccc}
\hline $\begin{array}{c}\mathrm{V}_{\mathrm{GBCA}} \\
( \pm 0.01 \mathrm{~mL})\end{array}$ & $\begin{array}{c}\mathrm{V}_{\mathrm{w}} \\
( \pm 20 \mathrm{~mL})\end{array}$ & $\begin{array}{c}{[\mathrm{Gd}] \pm \text { error }} \\
(\mathrm{mM})\end{array}$ \\
\hline 0.00 & -- & $0.00 \pm 0.00$ \\
0.10 & 1000 & $0.10 \pm 0.01$ \\
0.11 & 1000 & $0.11 \pm 0.01$ \\
0.26 & 1000 & $0.26 \pm 0.01$ \\
0.43 & 1000 & $0.43 \pm 0.01$ \\
0.30 & 500 & $0.60 \pm 0.03$ \\
0.77 & 1000 & $0.77 \pm 0.02$ \\
0.45 & 500 & $0.90 \pm 0.04$ \\
0.50 & 500 & $1.00 \pm 0.04$ \\
0.55 & 500 & $1.10 \pm 0.05$ \\
0.32 & 250 & $1.28 \pm 0.11$ \\
0.75 & 500 & $1.50 \pm 0.06$ \\
0.62 & 200 & $3.10 \pm 0.31$ \\
0.61 & 100 & $6.10 \pm 1.22$ \\
\hline
\end{tabular}




$$
\sigma_{[\mathrm{Gd}]}=[\mathrm{Gd}] \sqrt{\left(\frac{\sigma_{\mathrm{V}_{\mathrm{GBCA}}}}{\mathrm{V}_{\mathrm{GBCA}}}\right)^{2}+\left(\frac{\sigma_{\mathrm{V}_{\mathrm{w}}}}{\mathrm{V}_{\mathrm{w}}}\right)^{2}}
$$

\subsubsection{Dynamic Phantom}

A thin-tube aqueous multimodality DCE-MRI perfusion flow phantom (Shelley Medical, London, Canada) was used for the dynamic phantom experiments [82]. A schematic of the phantom exchange cylinder is shown in Fig. 5.2 and a schematic of the entire phantom system is shown in Fig. 5.3. The exchange cylinder was designed to simulate twocompartment contrast flow pharmacokinetics. The input and output tubing had a diameter of $10.0 \pm 0.5 \mathrm{~mm}$ and a total tubing length of about $5 \mathrm{~m}$. The system was filled with room-temperature tap water. The input and output tubing of the system was connected to a large reservoir of water $(\sim 1.5 \mathrm{~L})$ to simulate the rest of the human circulatory system. To ensure an even [Gd] throughout the reservoir and tubing system, the output and input tubing were positioned at opposite sides of the reservoir. The flow of water could be set manually, including the ability to stop the flow of water completely.

During the acquisition of each PO AIF, the flow was set between 140 and $250 \mathrm{~mL} \mathrm{~min}^{-1}$. Six AIF acquisitions were performed over a span of three days.

\subsubsection{Static Phantom $\mathrm{T}_{1}$ Measurements}

The primary method to obtain $T_{1}$ data in this work is by use of inversion recovery (IR) techniques. To do this, MR SI data are acquired at multiple points in time (where each point is known as an inversion time, $\mathrm{Tl}$ ) following an initial RF inversion pulse to create an 


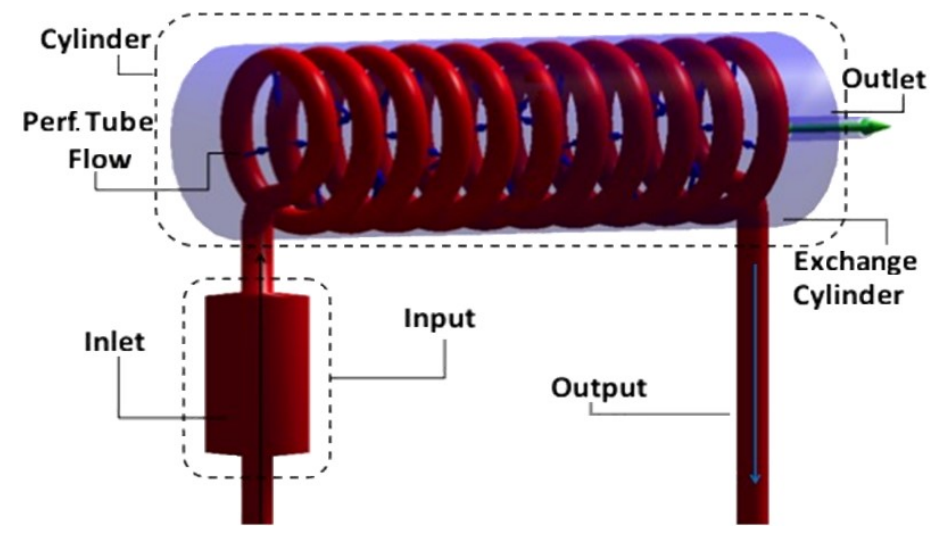

Figure 5.2: Schematic of the single-compartment system of the dynamic phantom [82]. AIF data were obtained by placing an ROI within the input tubing as it appeared on the images. The tubing of the input and output were connected to a reservoir of water to simulate the distribution of blood throughout the body. This illustration was used, with permission, by Gabrani-Juma et al. [83].

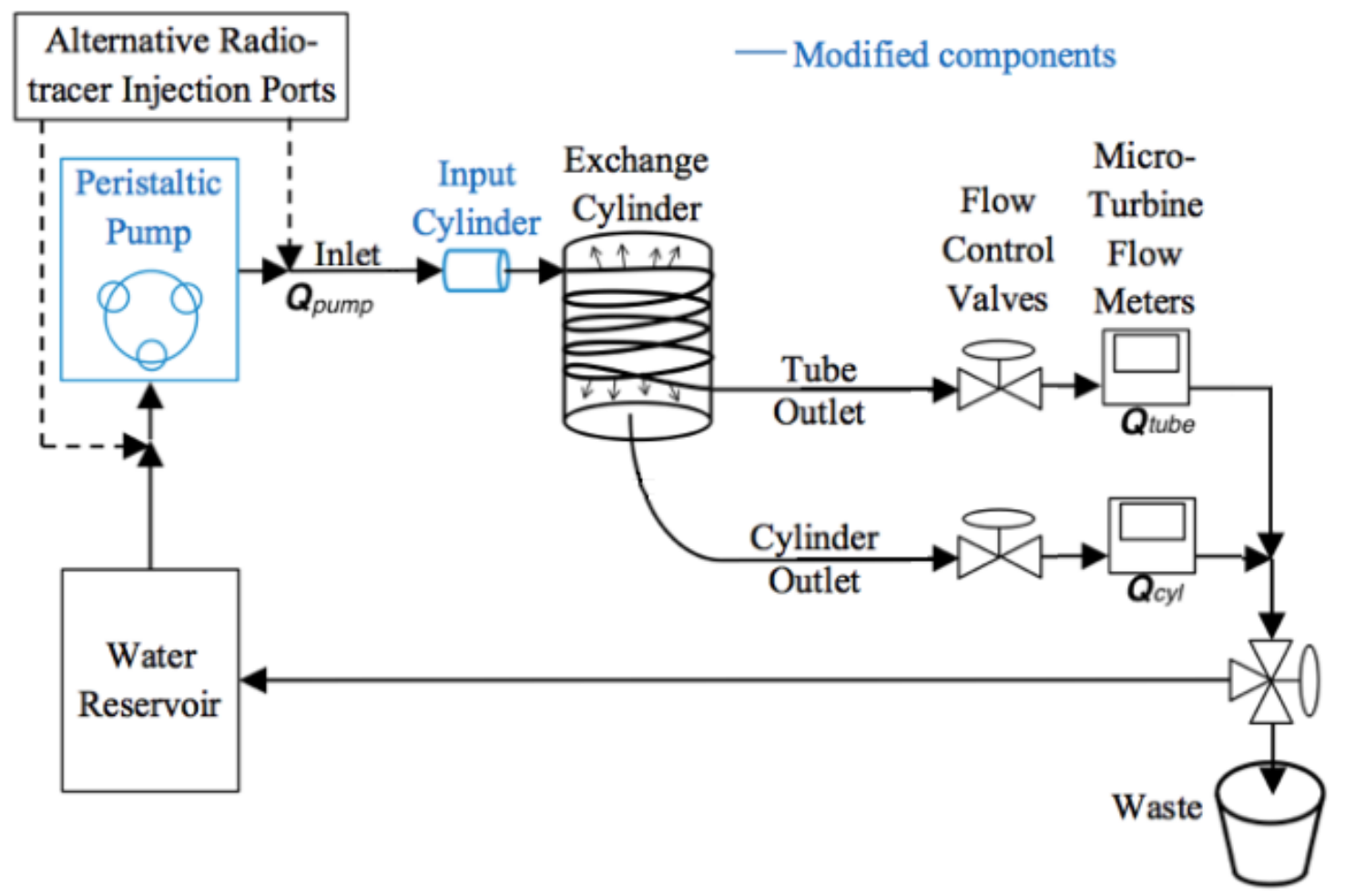

Figure 5.3: A schematic of the dynamic phantom system used during experiments. The blue components indicate modifications from the original phantom and pump system created by Shelley Medical for use in nuclear medicine, MR, and computed tomography. This image was used, with permission, by Gabrani-Juma et al. [83]. 
image at each TI data point.

The static tube phantoms were imaged on the Siemens scanner using two different sequences: echo-planar inversion recovery (EPI-IR, Section 2.4.5) and Modified Look-Locker Inversion Recovery (MOLLI, Section 2.4.6). EPI-IR data were acquired using the following parameter settings: $T_{E}=29 \mathrm{~ms}, T_{R}=6000 \mathrm{~ms}$, TI values $=35,60,100,150$, $250,500,750,1000,1250,1500,2000,3000,5000 \mathrm{~ms}, \alpha=90^{\circ}$, slice gap $=1 \mathrm{~mm}$, slice thickness $=5 \mathrm{~mm}, 18$ imaged slices, and scan time $=390 \mathrm{~s}$. MOLLI sequence parameters were: $\mathrm{T}_{\mathrm{E}}=1.08 \mathrm{~ms}, \alpha=30^{\circ}$, slice gap $=0 \mathrm{~mm}$, slice thickness $=5 \mathrm{~mm}, 18$ imaged slices, and eight equally-spaced $\mathrm{TI}$ points starting at $\mathrm{TI}=85 \mathrm{~ms}$ with constant $\mathrm{TI}$ spacing. Both EPIIIR and MOLLI data were acquired with a matrix size of $128 \times 128$ voxels with a field of view (FOV) of $220 \times 220 \mathrm{~mm}^{2}$. These two settings were chosen to match the resolution of the clinical DCE-MRI protocol. When the static phantoms were placed in the MR scanner, they were slightly tilted vertically $\left(<5^{\circ}\right)$ to remove air bubbles from the imaging volume in the centre of the phantom.

As mentioned in Section 2.4.6, the MOLLI sequence used for the work presented here was developed by Siemens for cardiac $T_{1}$ relaxation measurements. When imaging the heart, cardiac gating is necessary and the acquisition must be spread over more than one heart beat. Typically, three TI values are used for three consecutive heart beats for a total of nine different TI values with a non-uniform separation [34]. This sequence was modified for the brain measurements reported in Chapter 6 (and tested on phantoms in the current chapter) by removing cardiac gating and using $\mathrm{TI}$ values with a constant separation. 
The EPI-IR protocol was created to have large TI coverage to span the entire range of $T_{1}$ values expected in this study. Compared to EPI-IR, MOLLI utilized a much shorter $T_{R}$ and $\mathrm{TI}$ range such that only eight inversion points could be performed with each acquisition. This means that a single MOLLI sequence was designed to measure $T_{1}$ for a relatively narrow range of $[\mathrm{Gd}]$ values. The $\mathrm{TI}$ range and spacing were manually set by the user prior to the image acquisition. Therefore, to accurately compute [Gd] using $T_{1}$ values measured with MOLLI, some knowledge of the expected [Gd] within the imaged sample is required to choose the appropriate range of TI values for the MOLLI sequence. To accommodate the expected range of [Gd] values, two different TI spacing distributions were chosen for the MOLLI acquisitions.

For the measurement of lower [Gd] acquisitions (a longer $\mathrm{T}_{1}$ recovery) the $\mathrm{TI}$ spacing, which for MOLLI is also the $T_{R}$ value, was chosen to be $750 \mathrm{~ms}(\mathrm{TI}=85,835,1585$, $2335,3085,3835,4585$, and $5335 \mathrm{~ms})$ for a total scan time of 129 s. For higher [Gd] acquisitions (a shorter $\mathrm{T}_{1}$ recovery), the $\mathrm{Tl}$ spacing was chosen to be $175 \mathrm{~ms}(\mathrm{TI}=85,260$, $435,610,785,960,1135$, and $1310 \mathrm{~ms}$ ) for a total scan time of $30 \mathrm{~s}$. These MOLLI protocols were set up for the anticipated baseline (termed MOLLI ${ }_{0}$ ) and the washout (termed $\left.\mathrm{MOLLI}_{1}\right)_{1} \mathrm{~T}_{1}$ values, respectively. In this thesis, when $\mathrm{MOLLI}_{0}$ or $\mathrm{MOLLI}_{1}$ is not specified, the term MOLLI will be used as a generic description of the acquisition.

Due to their small volume, multiple static tube phantoms were imaged in a bundle in air. They were organized within the bundle to ensure there was enough space between each static tube phantom (a few $\mathrm{cm}$ ). This was done in order to minimize susceptibility effects between one phantom and another. 


\subsubsection{1 $\mathrm{T}_{1}$ Analysis Method}

The following analysis method was used on the measured SI data obtained from the static phantoms. SI inversion recovery curves were compiled on a voxel-by-voxel basis for each image. When the IR sequence is performed, the SI data immediately following the initial inversion pulse should be negative and then become positive at a time dictated by the $T_{1}$ of the sample. The MR scanner only acquires SI as a positive value, so a method to determine the proper signal polarity following an inversion pulse had to be established before $\mathrm{T}_{1}$ data could be extracted from the SI data. An example of SI data acquired in a static tube phantom with the EPI-IR and MOLLI 0 sequences is shown in Fig. 5.4.

To appropriately determine which SI values should be negative, a technique adapted from Nekolla et al. [84] was used. The algorithm used here was as follows: 1) the polarity of the first SI data point was inverted and $A, B$, and $T_{1}$ were fit to the data, as dictated by Eq. 2.77 for the EPI-IR sequence and Eq. 2.79 for the MOLLI sequences; 2 ) the Sl's of the first two data points were inverted and $A, B$, and $T_{1}$ were evaluated; 3 ) this process was repeated so that the Sl's of the first six data points were inverted. The fit with the smallest Root Mean Square Error (RMSE) of the seven sets of data (including data with no points inverted) was determined to be the method that most accurately fit for $T_{1}$. This algorithm was performed on a voxel-by-voxel basis with a three-parameter LevenbergMarquardt nonlinear curve fit with MATLAB 2017b (The MathWorks, Inc., Natick, United States). $T_{1}$ data were then averaged using a circular region of interest (ROI) in the centre of each phantom through the centre six slices to provide a single $T_{1}$ value and standard deviation for each phantom. The major benefit to the method of SI polarity selection is 


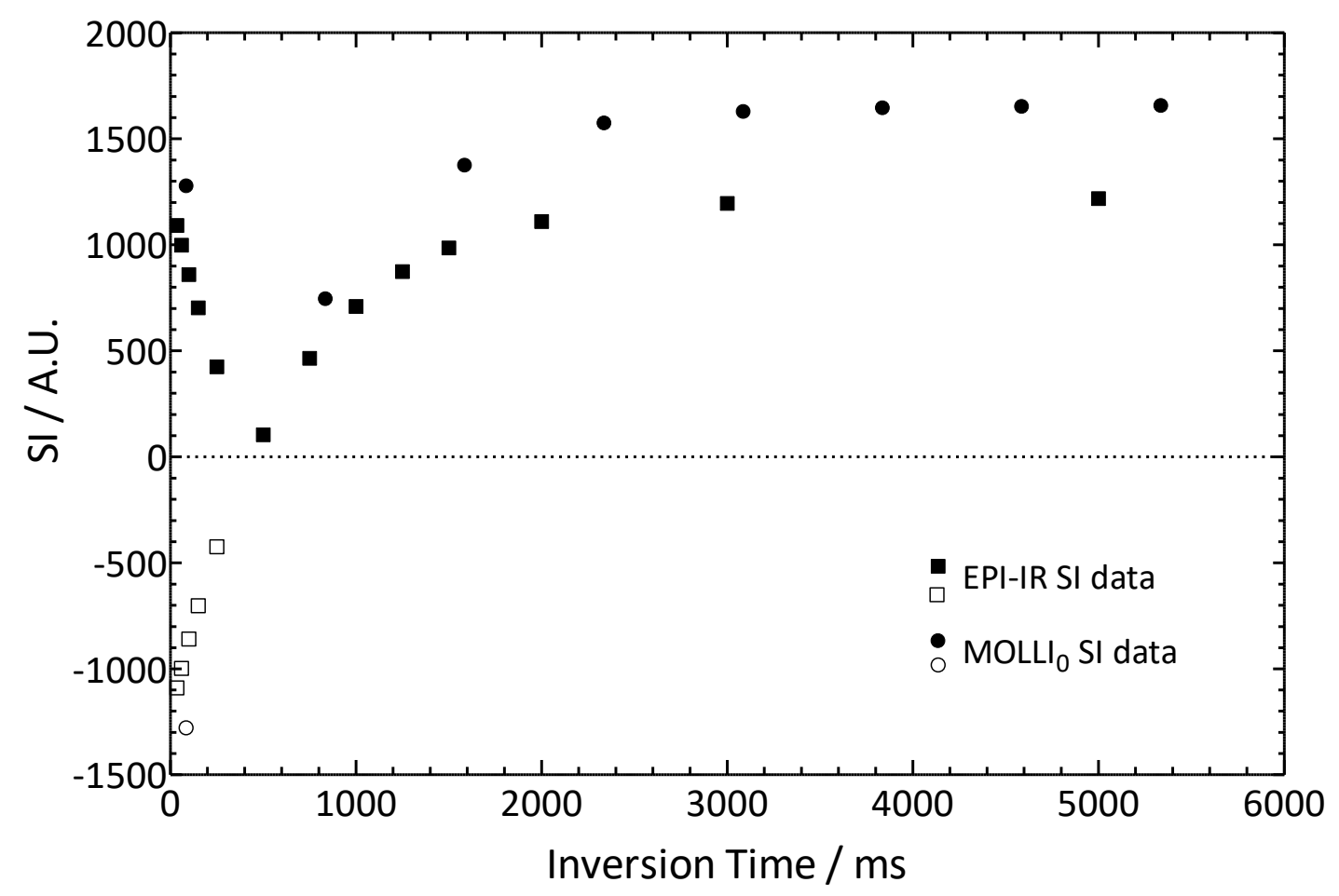

Figure 5.4: Example of IR SI curves following a single $180^{\circ}$ inversion pulse for both EPI-IR and $\mathrm{MOLLI}_{0}$ for the $0.26 \mathrm{mM}$ static phantom. The solid circles indicate the raw SI values outputted from the scanner, before SI polarity inversion. The hollow circles are the corrected signal data.

the removal of bias in the choice of which SI data points to invert based solely on the appearance of the data.

As described in Section 2.4.6, MOLLI does not directly measure the SI recovery of data on the IR curve dictated by the $T_{1}$ value, it traces the recovery due to the effective $T_{1}$, or $T_{1}^{*}$. The subsequent true $T_{1}$ value was then computed using Eq. 2.80 with $A, B$, and $T_{1}^{*}$ obtained from the fit of Eq. 2.79. Some $T_{1}^{*}$ and $T_{1}$ data from the $M O L L I_{0}$ sequences were removed from the analysis as the $\mathrm{TI}$ values used were not appropriately chosen to properly characterize the change in SI for high [Gd] phantoms. MOLLI ${ }_{1}$ for low [Gd] 
phantoms was rejected for similar reasons. Examples of the MR signal measured with $\mathrm{MOLLI}_{0}$ for the $1.50 \mathrm{mM}$ phantom and $\mathrm{MOLL}_{1}$ performed for the $0.1 \mathrm{mM}$ phantom are shown in Fig. 5.5. A fit to these data would not be considered accurate enough due to the spacing of TI data points. It was decided that, for a fit to be considered accurate, at least two data points in the range of $0.5 \mathrm{~T}_{1} \leq \mathrm{Tl} \leq 3 \mathrm{~T}_{1}$ were required. For the $1.50 \mathrm{mM}$ data, most of the TI values correspond to the equilibrium part of the function rather than the curved part which is critical for properly characterizing the exponential growth. For the $0.1 \mathrm{mM}$ phantom, there are no data points in the equilibrium region.

The measured $T_{1}$ values were used to establish a [Gd] calibration curve using Eq. 2.84. In this equation, the longitudinal relaxivity value, $r_{1}$, is the slope and the zero-[Gd] $T_{1}$ value, $T_{1,0}$, is the $y$-intercept. The $r_{1}$ value for gadobutrol in room-temperature water can be easily computed as all the data in Eq. 2.84 are known. Determining the $r_{1}$ value allows for the calculation of the [Gd] value of any sample once its $T_{1}$ value is acquired and a $T_{1,0}$ value is known ( $T_{1,0}$ can also be measured). Note, Eq. 2.84 was also used for the $T_{1}^{*}$ and $T_{1,0}^{*}$ values obtained in the MOLLI sequences with a corresponding $r_{1}^{*}$ value. $T_{1}$ and $r_{1}$ values obtained from all $\mathrm{T}_{1}$ sequences were compared. The fast MOLLI sequence was also compared to the slower, more accurate EPI-IR sequence to determine its validity for clinical data acquisitions.

\subsubsection{Dynamic Phantom Measurements}

The dynamic phantom was used to simulate the PO AIF obtained in a clinical DCE-MRI acquisition and to construct the PT AIF using the same techniques for the $T_{1}$ measurement 


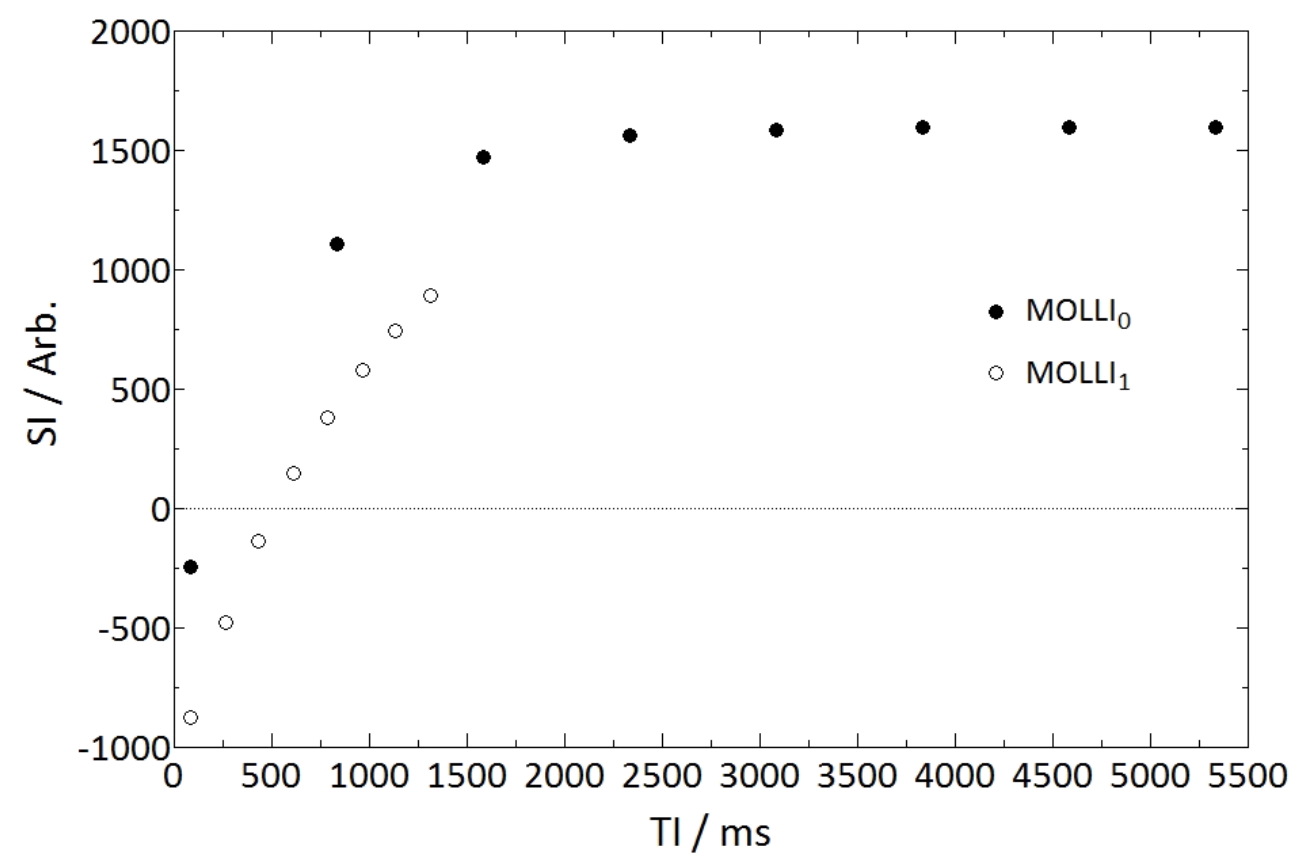

Figure. 5.5: An example of the inadequate TI spacing of $\mathrm{MOLLI}_{0}$ to sample the SI of a high (1.5 $\mathrm{mM})[\mathrm{Gd}]$ phantom and $\mathrm{MOLLI}_{1}$ to sample the $\mathrm{SI}$ of a low $(0.1 \mathrm{mM})[\mathrm{Gd}]$ phantom.

and analysis as were used for the static phantoms. This process was as follows: $T_{1}$ was measured for the baseline, a PO AIF was acquired after a bolus of GBCA was injected, $T_{1}$ was measured for the washout, and finally Eq. 2.84 was used with the $r_{1}$ value determined in static phantom experiments to compute the [Gd] for the washout and baseline. This process is described in detail below.

Although water is often a useful substitute for blood in experimental science, it has many properties that are different from blood. Most relevant here is the difference in their $T_{1}$ values. To better simulate the $T_{1}$ of blood in the baseline of the AIF, a small amount of GBCA (about $0.1 \mathrm{mmol}$ ) was added to the water so that the measured value for $T_{1,0}$ was comparable to the $T_{1}$ of venous blood in adults at $3 \mathrm{~T}(1650-1932 \mathrm{~ms}[70,85])$. 
To ensure there was a uniform [Gd] throughout the phantom system, the phantom was set to flow for several minutes and multiple $T_{1}$ measurements were performed using MOLLI 0 . The $T_{1}$ value was deemed stable when it stopped decreasing between two consecutive acquisitions. EPI-IR and MOLLI were performed both with the water flow shut off and with the water flowing. $T_{1,0}$ values were compared between these two flow settings to observe the effect that water flow has on $T_{1}$ measurements. As an additional check, water was drawn from the phantom reservoir and placed into a small cup phantom with a volume of $90 \mathrm{~mL}$ and a diameter of $48 \mathrm{~mm}$. Both EPI-IR and MOLLI sequences were then performed on these 'cup' phantoms. In all instances, $T_{1,0}$ values were computed from the SI data using the same technique as described for the static phantoms.

With the water set to flow, the DCE-MRI sequence was performed using a Fast Low Angle Shot (FLASH) sequence with parameter settings: $T_{R}=6.5 \mathrm{~ms}$, temporal resolution $=3.5 \mathrm{~s}, \alpha=30^{\circ}$, matrix size $=128 \times 128$ pixels, FOV $=220 \times 220 \mathrm{~mm}^{2}$, and double $T_{E}=1.65 \mathrm{~ms}$ and $3.85 \mathrm{~ms}$. The PO AIF was calculated from the $\phi$ data of the second echo $(3.85 \mathrm{~ms})[47,86]$. The first echo $(1.65 \mathrm{~ms})$ data are used in the calculation of the tissue transport function when the sequence is used clinically. A short echo time is required in this case to minimize $T_{2}^{*}$ effects. The second echo was used to acquire PO AIF data since a larger $T_{E}$ will allow for greater phase accumulation. The short echo time data were only used clinically since a tissue transport function could not be measured for the phantoms. To match the $T_{1}$ imaging set up, 18 contiguous slices were acquired at a thickness of $5 \mathrm{~mm}$. FLASH SI and $\phi$ data were acquired for $10-15 \mathrm{~s}$ to establish the baseline of the PO AIF. A bolus of GBCA ( 1 mL) was injected into a designated port downstream 
from the main pump system. The injection was performed by hand and took approximately two to three seconds. Following the injection of GBCA, along with the GBCA put into the system at the baseline, the [Gd] of the dynamic phantom was expected to reach an equilibrium value of about $0.7 \pm 0.1 \mathrm{mM}$ in the washout for each acquisition. Note that the stated error for this washout value is a conservative estimate.

Phase data, $\phi(t)$, were continuously acquired as the bolus of GBCA passed through the phantom system by repeating the 3D FLASH sequence until [Gd] equilibrium was achieved, i.e. the washout. The FLASH sequence was repeated continuously for $10-15$ min to allow for a full acquisition of the AIF. Average phase data values were obtained for the phantom input tube using an ROI on the center slices. The ROI was just smaller than the size of the input tube of the phantom. The acquired phase dataset was unwrapped on a voxel-by-voxel basis across time, a process that verifies that the change in phase between two consecutive time points was between $\pm \pi$ rad to prevent "phase aliasing" $[52,65]$. $[G d]$ as a function of time, $[G d](t)$, was computed from the unwrapped phase data using Eq. 2.109 to create the PO AIF. Care was taken when setting up the dynamic phantom to make sure the input tubing was parallel to $\mathbf{B}_{\mathbf{0}}$ so that the geometry factor defined by Eq. 2.110 simplifies to $f(\theta)=1 / 3$. An example of a typical AIF acquired from the dynamic phantom system is shown in Fig. 5.6. These measurements were repeated twice on three different days with different quantities of GBCA and water used each time. This was done to ensure the AIF could be measured clinically at the various [Gd] values expected in a typical DCE-MRI acquisition.

EPI-IR and MOLLI were again performed with the water flowing and static after 


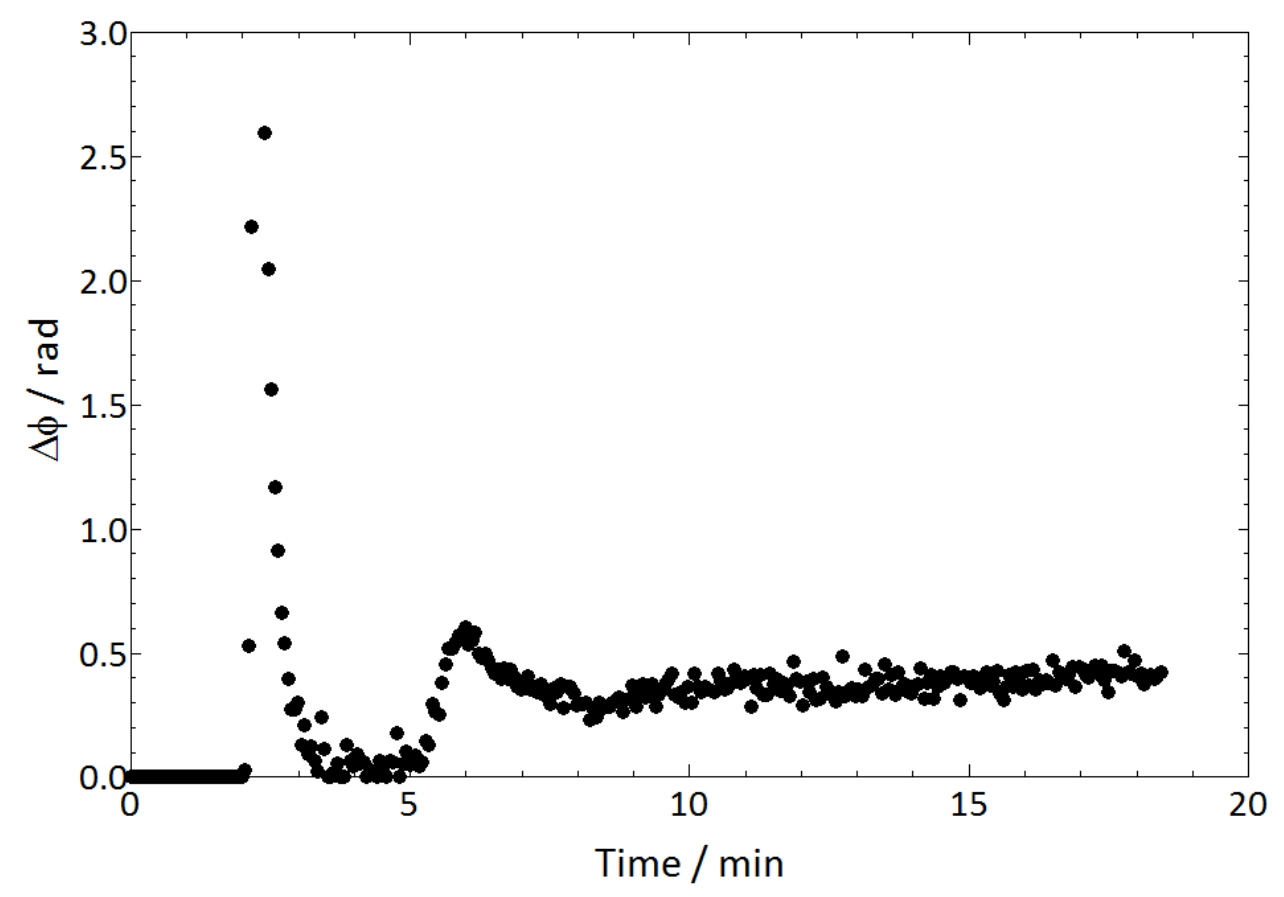

Figure 5.6: Example of a dynamic phantom AIF. It is characterized by the initial, baseline phase values, a high peak following GBCA injection, a local minimum, a second peak due to recirculation of the GBCA, followed by steady-state washout values.

[Gd] equilibrium was reached to measure $T_{1}$ in the washout, termed $T_{1, w}$. Just like for the baseline, water was drawn from the reservoir and placed into the cup phantom where $T_{1, w}$ was measured to confirm the measurement technique in the dynamic phantom. For each AIF acquisition, both $\mathrm{MOLLI}_{0}$ and $\mathrm{MOLLI}_{1}$ were performed on the cup phantom for the washout. This was done as the expected washout [Gd] was not well known. To ensure proper TI coverage, both MOLLI sequences were performed. The sequence with the smallest RMSE for the $T_{1}$ fit equation was determined to be the more accurate method for that $[G d]$. The $T_{1, w}$ and $T_{1,0}$ values were then used to calculate the AlF value for the washout, AlF $_{w}$, with the $r_{1}$ value obtained from static phantom experiments using Eq. 4.12 
both in the dynamic and cup phantoms. The AlF $_{\mathrm{w}}$ value, combined with the PO AIF, allows for the creation of the PT AIF using Eq. 4.4. $T_{1}$ values computed from the EPI-IR data were used as the gold standard. The $A_{\mathrm{W}}$ values were subsequently compared using a paired $t$-test.

\subsection{Results}

\subsubsection{Static Phantom Results}

$T_{1}$ and $T_{1}^{*}$ fit values are shown in Table 5.2 for the static phantoms. Note that the $T_{1}$ of the two high [Gd] phantoms (3.1 and $6.1 \mathrm{mM}$ ) could not be measured accurately due to their extremely fast $T_{1}$ recovery and are excluded from analysis. Other $T_{1}$ values were excluded based on the relationship between $\mathrm{T}_{1}$ relaxation rate and the $\mathrm{TI}$ values used as discussed in Section 5.2.2.1. Excluded values are indicated by '--' in Table 5.2. Excluded data were not used in subsequent $r_{1}$ and $r_{1}^{*}$ calculations.

The conversion of $T_{1}^{*}$ to $T_{1}$ typically results in a larger $T_{1}$ value than $T_{1}^{*}$. From Table 5.2 , this is observed, except at low concentrations for MOLLI ${ }_{1}$. The phantom data show that the $T_{1}^{*}$ data measured using the two MOLLI sequences were more accurate when compared to the gold standard EPI-IR $\mathrm{T}_{1}$ data.

From the computed $T_{1}$ and $T_{1}^{*}$ data, $[\mathrm{Gd}$ ] calibration curves were created to allow the determination of $[\mathrm{Gd}]$ values from any measured $T_{1}$ values. This process assumes that a known $r_{1}$ value exists, so it was first calculated from the known [Gd] phantoms and computed $T_{1}$ values using Eq. 2.84. The $1 / T_{1}$ data vs. [Gd] are presented in Figs. 5.7 to 5.9 
Table 5.2: Summary of $T_{1}$ and $T_{1}^{*}$ values and standard deviations from 12 static phantoms. Data are marked in terms of their statistical significant difference from the EPI-IR data using an unpaired $t$-test: ${ }^{* * *} P \leq 0.001, * * P \leq 0.01$, and $* P \leq 0.05$.

\begin{tabular}{cccccc}
\hline$[\mathrm{Gd}]$ & EPI-IR & $\mathrm{MOLLI}_{0}$ & $\mathrm{MOLLI}_{1}$ & $\mathrm{MOLLI}_{0}$ & $\mathrm{MOLLI}_{1}$ \\
$(\mathrm{mM})$ & $\mathrm{T}_{1}(\mathrm{~ms})$ & $\mathrm{T}_{1}^{*}(\mathrm{~ms})$ & $\mathrm{T}_{1}^{*}(\mathrm{~ms})$ & $\mathrm{T}_{1}(\mathrm{~ms})$ & $\mathrm{T}_{1}(\mathrm{~ms})$ \\
\hline 0.00 & $2887 \pm 67$ & $2959 \pm 56^{* * *}$ & -- & $2407 \pm 14^{* * *}$ & -- \\
0.10 & $1005 \pm 11$ & $1010 \pm 7^{*}$ & $1006 \pm 33$ & $1021 \pm 11^{* * *}$ & $680 \pm 8^{* * *}$ \\
0.11 & $843 \pm 8$ & $852 \pm 9^{* *}$ & $847 \pm 20$ & $864 \pm 12^{* * *}$ & $641 \pm 9^{* * *}$ \\
0.26 & $640 \pm 6$ & $639 \pm 7$ & $635 \pm 12$ & $656 \pm 8^{* * *}$ & $561 \pm 9^{* * *}$ \\
0.43 & $403 \pm 4$ & -- & $403 \pm 9$ & -- & $412 \pm 8^{* * *}$ \\
0.60 & $272 \pm 3$ & -- & $273 \pm 8$ & -- & $295 \pm 8^{* * *}$ \\
0.77 & $257 \pm 3$ & -- & $257 \pm 7$ & -- & $281 \pm 8^{* * *}$ \\
0.90 & $203 \pm 3$ & -- & $202 \pm 7$ & -- & $228 \pm 6^{* * *}$ \\
1.00 & $182 \pm 3$ & -- & $182 \pm 6$ & -- & $207 \pm 6^{* * *}$ \\
1.10 & $179 \pm 3$ & -- & $178 \pm 6$ & -- & $204 \pm 6^{* * *}$ \\
1.28 & $140 \pm 3$ & -- & $137 \pm 5$ & -- & $166 \pm 4^{* * *}$ \\
1.50 & $131 \pm 2$ & -- & $130 \pm 6$ & -- & $159 \pm 4^{* * *}$ \\
\hline
\end{tabular}

as follows: EPI-IR data (Fig. 5.7), MOLLI T1 $T_{1}^{*}$ data (Fig. 5.8), and MOLLI $T_{1}$ data (Fig. 5.9).

The $r_{1}$ and $r_{1}^{*}$ values from these plots and the Pearson correlation coefficient $(r)$ of the linear fit are displayed in Table 5.3. For gadobutrol in room-temperature water and at a field strength of $3 T, r_{1}$ was determined previously by Stalder et al. [43] to be $4.7 \pm$ $0.2 \mathrm{mM}^{-1} \mathrm{~s}^{-1}$. The EPI-IR value for $r_{1}$ reported here is in very good agreement with a $5.1 \%$ error when compared to Stalder's value. The $r_{1}$ value determined from the $\mathrm{MOLLI}_{1} \mathrm{~T}_{1}^{*}$ results is essentially equal to the EPI-IR $r_{1}$ value. The $r_{1}^{*}$ value obtained from the MOLLI $\mathrm{T}_{1}^{*}$ results is reasonable but it is considered to be less reliable since there are fewer data points for $\mathrm{MOLLI}_{0}$ and they cover a relatively small range of $[\mathrm{Gd}]$ values. For both $\mathrm{MOLLI}_{0}$ and $\mathrm{MOLLI}_{1}$, the values of $T_{1}$ and the $r_{1}{ }^{\prime}$ s calculated from them are not in good agreement with the corresponding EPI-IR values.

It can be seen from Table 5.2 that $\mathrm{MOLLI}_{0}$ and $\mathrm{MOLLI}_{1} \mathrm{~T}_{1}^{*}$ values were much more 


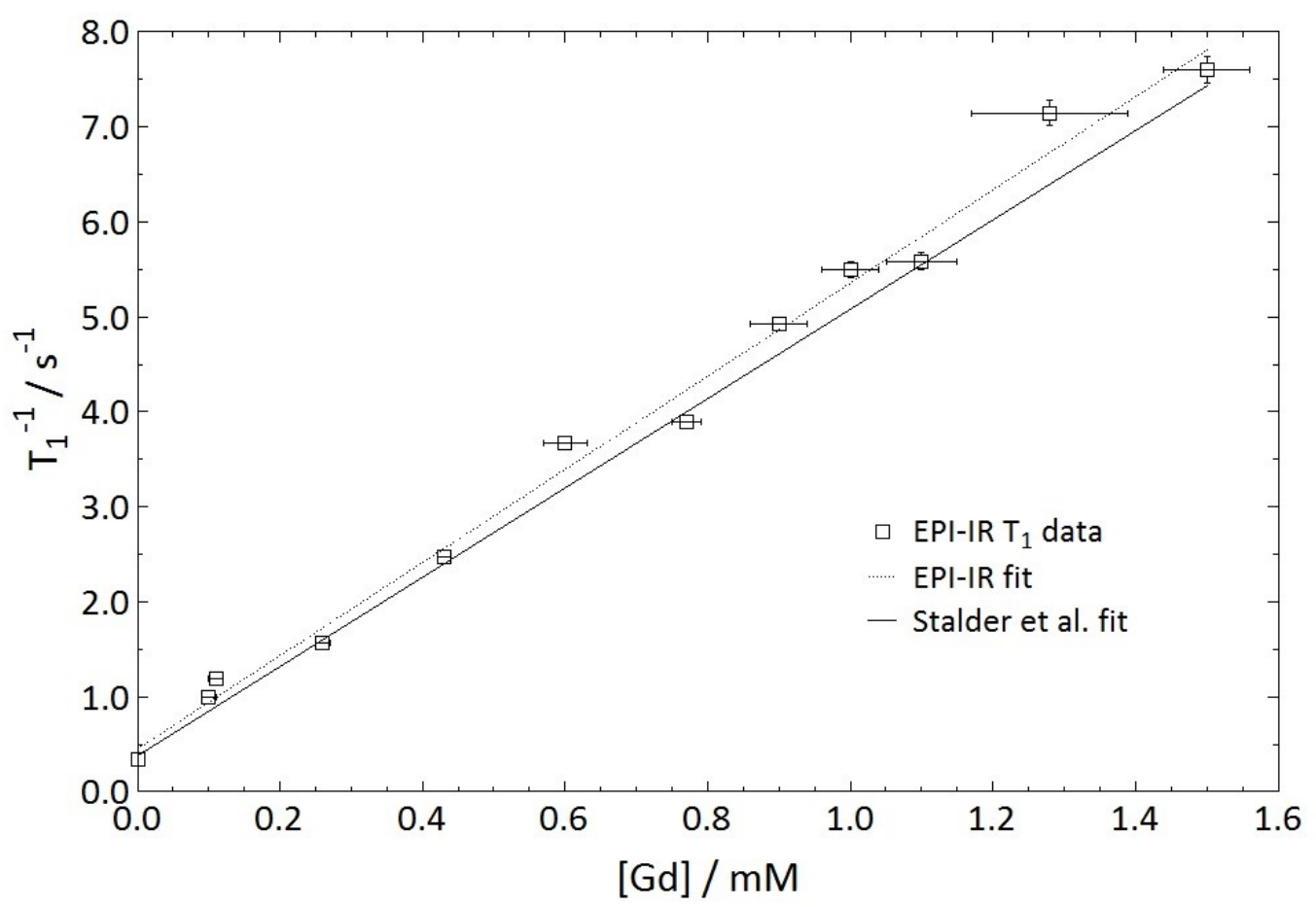

Figure 5.7: IR data acquired from static phantoms compared to the literature fit data from Stalder et al. [43] (solid line).

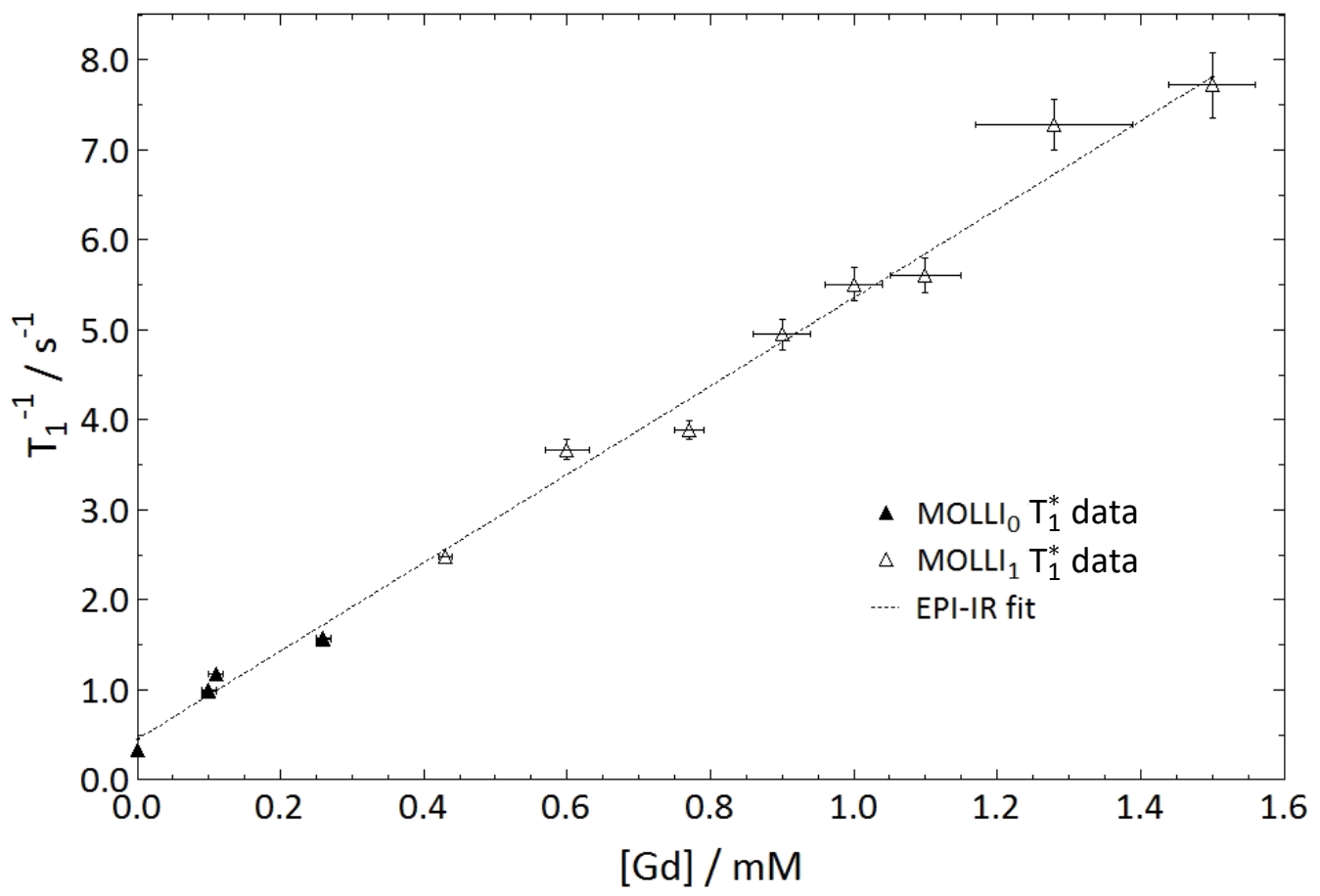

Figure 5.8: $\mathrm{MOLLI}_{0}$ and $\mathrm{MOLLI}_{1} \mathrm{~T}_{1}^{*}$ data acquired for static phantoms plotted with the EPIIR $\mathrm{T}_{1}$ line of best fit. 


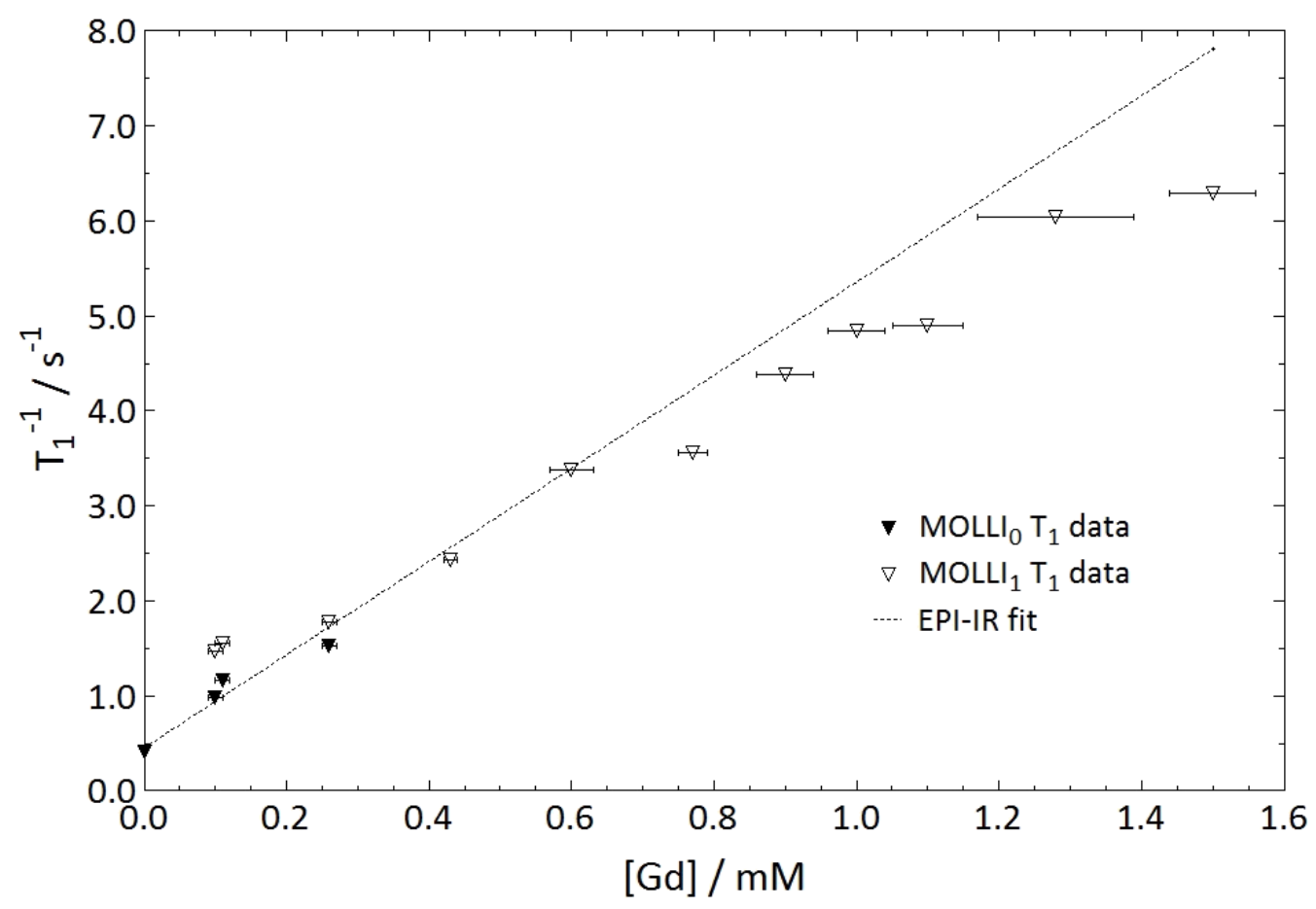

Figure 5.9: $\mathrm{MOLLI}_{0}$ and $\mathrm{MOLLI}_{1} \mathrm{~T}_{1}$ data acquired for static phantoms plotted with the EPIIR $T_{1}$ line of best fit.

Table 5.3: $r_{1}, r_{1}^{*}$, and Pearson correlation values computed for each $T_{1}$ method used.

\begin{tabular}{ccc} 
Data & $r_{1}$ or $r_{1}^{*}\left(\mathrm{mM}^{-1} \mathrm{~s}^{-1}\right)$ & $r$ \\
\hline EPI-IR T & $4.90 \pm 0.15$ & 0.996 \\
MOLLI $_{0} \mathrm{~T}_{1}^{*}$ & $4.55 \pm 1.01$ & 0.954 \\
MOLLI $_{1} \mathrm{~T}_{1}^{*}$ & $4.94 \pm 0.18$ & 0.994 \\
MOLLI $_{0} \mathrm{~T}_{1}$ & $4.14 \pm 0.86$ & 0.960 \\
MOLLI $_{1} \mathrm{~T}_{1}$ & $3.65 \pm 0.14$ & 0.993 \\
\hline
\end{tabular}


similar to EPI-IR $T_{1}$ values than the "corrected" MOLLI $T_{1}$ values based on the results of an unpaired $t$-test. It can also be seen quite clearly in Figs. 5.8 and 5.9. This was true over the entire range of $[G d]$ values for both MOLLI sequences. None of the $\mathrm{MOLLI}_{1} \mathrm{~T}_{1}^{*}$ values were significantly different from the EPI-IR $\mathrm{T}_{1}$ values, as measured by an unpaired $t$-test. This suggests that, for the static phantoms, MOLLI $T_{1}^{*}$ data does not need correction. Therefore, for the rest of the MOLLI phantom data used in this thesis, $T_{1}^{*}$ data were used as the proper values for $T_{1}$ and no corrections were applied.

Since $\mathrm{MOLLI}_{0}$ and $\mathrm{MOLLI}_{1}$ were designed for different [Gd] ranges, the results for these two sequences were combined into a single calibration curve. This new calibration method, termed $M O L L I_{\text {comb }}$, uses $1 / T_{1}^{*}$ values from the $M O L L I_{0}$ curve for low $[G d]$ values $(0.0-0.26 \mathrm{mM})$ and $\mathrm{MOLLI}$ data for the remaining $1 / T_{1}^{*}$ values. The $r_{1}^{*}$ values for $\mathrm{MOLLI}_{1}$ and $\mathrm{MOLLI}_{\text {comb }}$ are very similar $\left(\mathrm{MOLLI}_{\mathrm{comb}}: \mathrm{r}_{1}=4.98 \pm 0.15 \mathrm{mM}^{-1} \mathrm{~s}^{-1} ; \mathrm{MOLLI}_{1}: \mathrm{r}_{1}=\right.$ $4.94 \pm 0.18 \mathrm{mM}^{-1} \mathrm{~s}^{-1}$ ) and both are in excellent agreement with the $r_{1}$ obtained using EPIIR $\left(\%\right.$ error $=1.6 \%$ for MOLLI $\left._{\text {comb }}\right)$. From this point forward, all $[G d]$ data for phantoms with room-temperature water will be obtained using the $\mathrm{MOLLI}_{\text {comb }}$ calibration and $\mathrm{MOLLI}_{\text {comb }}$ will be denoted simply as "MOLLI", unless otherwise specified.

\subsubsection{Dynamic Phantom Results}

A total of six AIF trials were performed using the dynamic phantom. While the exact, initial [Gd] is not relevant for the PO or PT AIF computation methods, it was found to be approximately $0.07 \mathrm{mM}$ for each of the trials. These values were based on measurements from static cups filled with water taken from the phantom reservoir. Due to significant 
artefacts, the EPI-IR data could not be used for measurements taken within the dynamic phantom so EPI-IR was used only for the cup phantoms.

Images of the dynamic phantom were acquired using MOLLI for both flowing and static water. To observe the effect of water flow on computed $T_{1}^{*}$ measurements, a metric termed the 'flow ratio', $F_{R}$, was used:

$$
F_{R}=\frac{T_{1}^{*}(\text { flow on })}{T_{1}^{*}(\text { flow off })}
$$

$F_{R}$ values for two AIF trials are shown in Fig. 5.10. Since MOLLI ${ }_{0}$ was designed for the longer $\mathrm{T}_{1}$ 's expected when $[\mathrm{Gd}]=0$ (i.e. the baseline) and $\mathrm{MOLLI}_{1}$ was designed for shorter $\mathrm{T}_{1}^{\prime} \mathrm{s}, \mathrm{F}_{\mathrm{R}}$ results for $\mathrm{MOLLI}_{0}$ and $\mathrm{MOLLI}_{1}$ are reported only for the baseline and washout, respectively. From Fig. 5.10, it can be seen that the $T_{1}^{*}$ values obtained using MOLLI are not affected by flow. Note that, for $\mathrm{MOLLI}_{0}$ the average of the two trials was $0.98 \pm 0.4$.

The six acquired PO AIFs obtained using the dynamic phantom are displayed in Fig.

5.11. Each AIF was shifted in time such that the AIF peak values all occurred at the same time point ( $2.29 \mathrm{~min})$ and was truncated to end at the same time point (18.42 min). Since the data were measured using the same phantom system with the same imaging parameters, the AlFs were averaged to form a single acquisition that depicts a representative PO AIF. While the start of the washout region will vary depending on the flow rate of the phantom (as seen in Fig. 5.11), the washout of the average PO AIF was taken to start at approximately the 13 minute mark. From that time to the end of data collection, the slope for the average AIF was $-0.005 \mathrm{mM} \mathrm{min}^{-1}$, indicating a stable washout [Gd] value. The baseline phase, $\phi_{0}$, was then used to compute the PO AIF in terms of [Gd] with Eq. 2.109 after phase-unwrapping. 


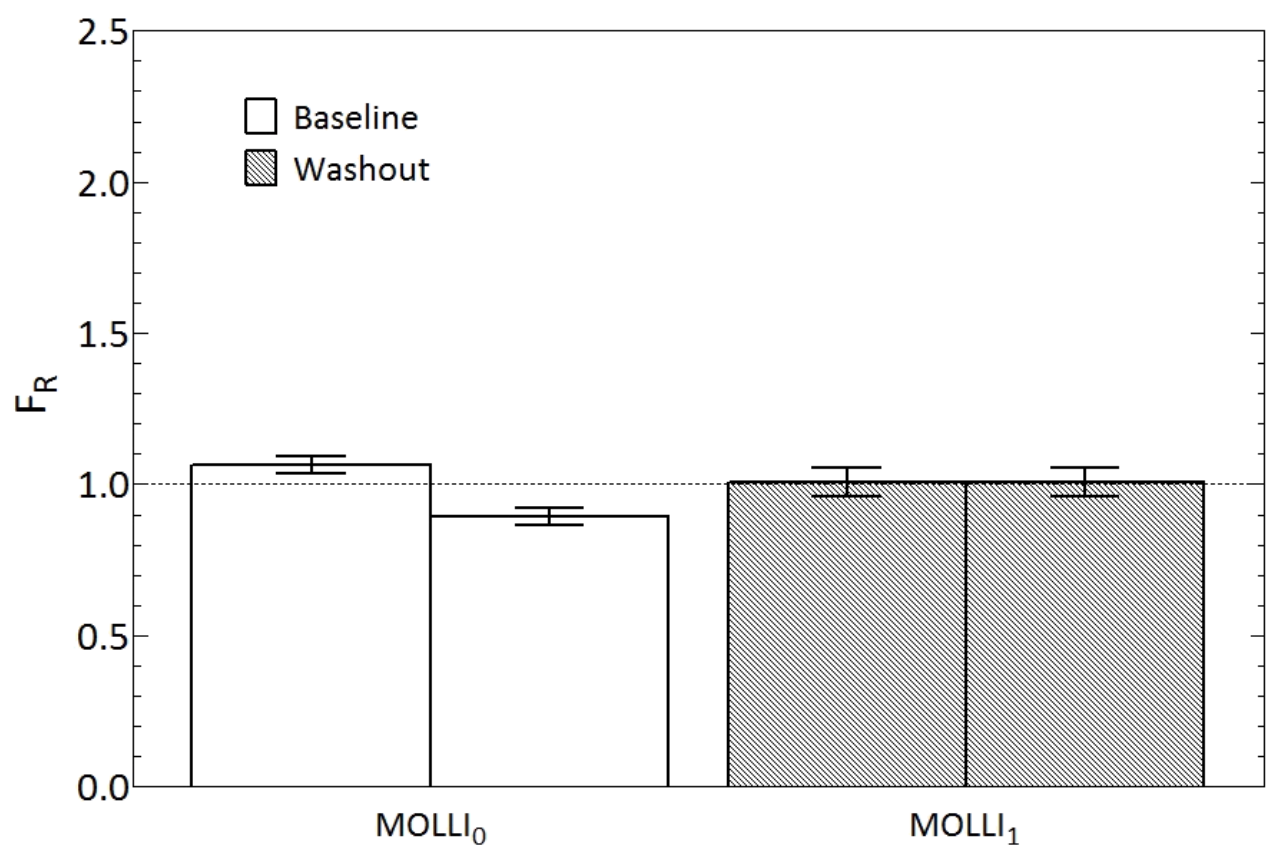

Figure 5.10: The flow ratio acquired using $\mathrm{MOLLI}_{0}$ and $\mathrm{MOLLI}_{1}$ from the baseline and washout, respectively, for two different measurements. Each column represents the average $T_{1}^{*}$ value over multiple slices and the error bars represent the standard deviation. The horizontal dashed line indicates a constant measurement of $\mathrm{T}_{1}^{*}$ between the two flow states.

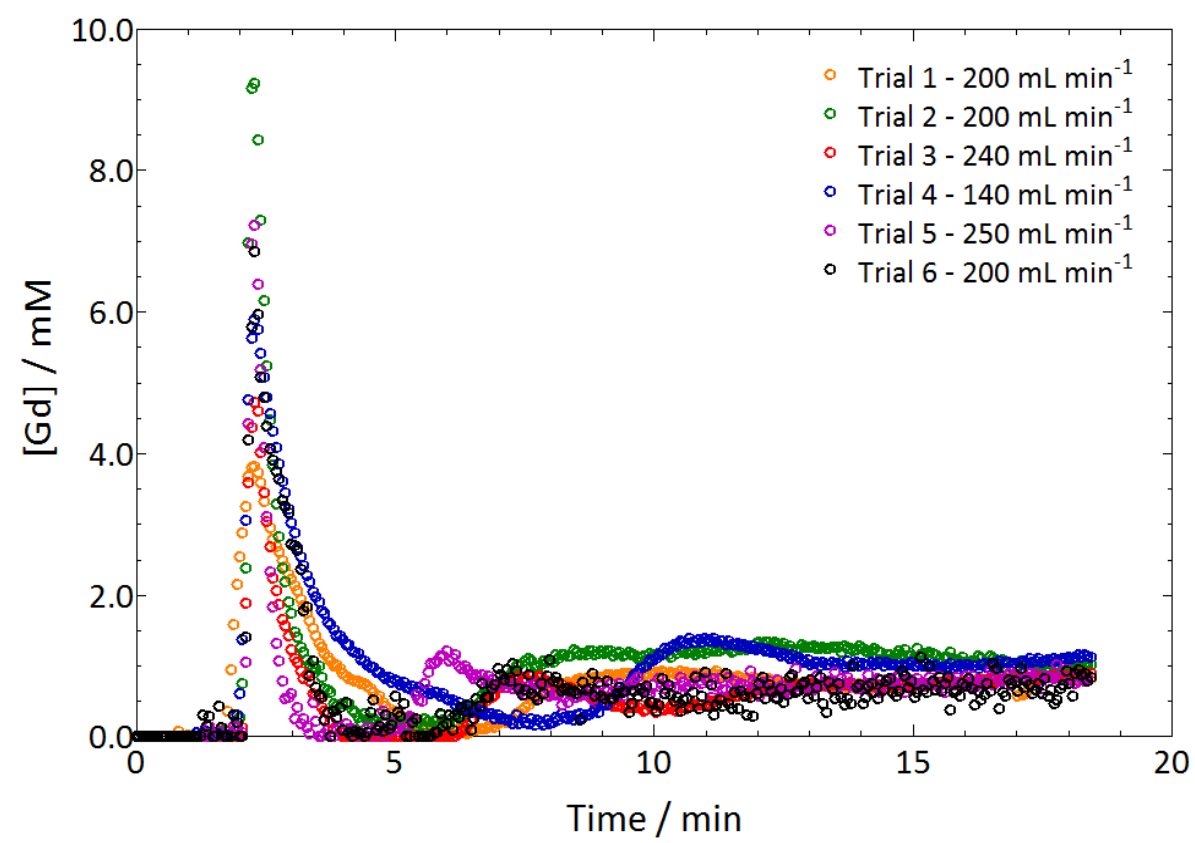

Figure 5.11: Six different PO AIFs measured using the dynamic phantom system along with the set flow rate. The AIFs were shifted in time so that the peak value occurred at the same time. 
After each AIF acquisition was completed, a $90 \mathrm{~mL}$ sample of fluid was drawn from the dynamic phantom system to measure $T_{1}$ from a static phantom (referred to as a cup phantom here), by the same methods stated previously for static phantoms using both MOLLI and EPIIIR. The AIF washout $[G d]$ value, $A F_{w}$, was then computed from the washout $T_{1}$ value using Eq. 2.84 and compared to the washout [Gd] values of the PO AlF. These results are shown in Fig. 5.12 and Table 5.4. From the graph, it can be seen that EPI-IR and MOLLI [Gd] values are well correlated in this concentration range $(r=0.995, P$ $<0.001)$. The phase data are not correlated $(r=0.067, P=0.898)$, indicating that at least for this concentration range, the values of [Gd] obtained using MOLLI are much more accurate than those computed from the signal phase.

Using the $\phi_{\mathrm{w}}$ value determined from the average PO AIF and the process described in Section 4.2, the PT AIF was created using the AIF $_{w}$ value calculated from the MOLLI $T_{1, w}^{*}$ and $T_{1,0}^{*}$ values. A representative PT AIF for the dynamic phantom is shown with the corresponding PO AIF in Fig. 5.13. In addition to the two curves, the EPI-IR AIF value measured using the static cup phantoms is shown on the graph for comparison. This measurement was conducted at a later point in time and is placed on the graph here only for a visual comparison.

For each acquisition, the PT AIF ${ }_{\mathrm{w}}$ values were significantly lower $(P<0.0001)$ than the PO AlF ${ }_{w}$ values. These PT AlF ${ }_{w}$ values determined using MOLLI were in very good agreement with the gold-standard AIF $_{w}$ values obtained with the EPI-IR $T_{1}$ measurements. The fact that no significant difference was observed between the MOLLI and EPI-IR $T_{1}$ measurements $(P=0.096)$ provides confidence that the PT AlF ${ }_{\mathrm{w}}$ values measured with 


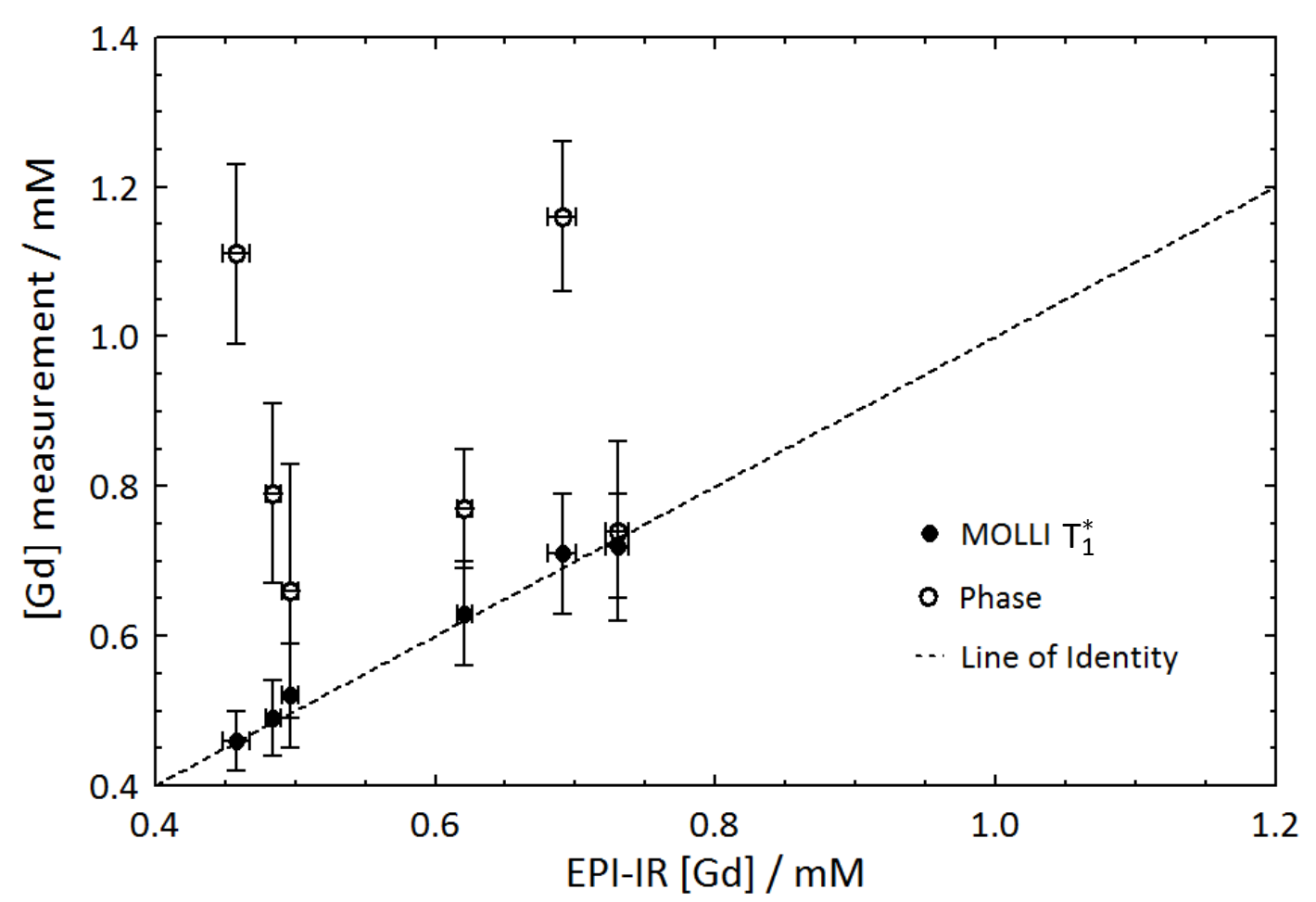

Figure 5.12: Comparison of [Gd] measurement techniques for concentrations measured in the AIF washout. MOLLI T1 data (using MOLLI $_{\text {comb }}$ ) correlated well with the EPI-IR data.

Table 5.4: Dynamic phantom washout [Gd] values and standard deviations measured using the phase and MOLLI comb $T_{1}$ methods. The values obtained from phase and $T_{1}$ are denoted $[\mathrm{Gd}]_{\phi}$ and $[\mathrm{Gd}]_{T_{1}}$, respectively. The final column indicates the difference in the two previous columns.

\begin{tabular}{cccc}
\hline AIF Acquisition & {$[\mathrm{Gd}]_{\phi} \pm \mathrm{SD}(\mathrm{mM})$} & {$[\mathrm{Gd}]_{\mathrm{T}_{1}} \pm \mathrm{SD}(\mathrm{mM})$} & Difference $(\mathrm{mM})$ \\
\hline 1 & $0.79 \pm 0.12$ & $0.49 \pm 0.05$ & -0.30 \\
2 & $1.16 \pm 0.10$ & $0.71 \pm 0.08$ & -0.45 \\
3 & $0.74 \pm 0.12$ & $0.72 \pm 0.07$ & -0.02 \\
4 & $1.11 \pm 0.12$ & $0.46 \pm 0.04$ & -0.65 \\
5 & $0.77 \pm 0.08$ & $0.63 \pm 0.07$ & -0.14 \\
6 & $0.66 \pm 0.17$ & $0.52 \pm 0.07$ & -0.14 \\
\hline Average & $0.87 \pm 0.05$ & $0.59 \pm 0.03$ & $-0.28 \pm 0.23$
\end{tabular}




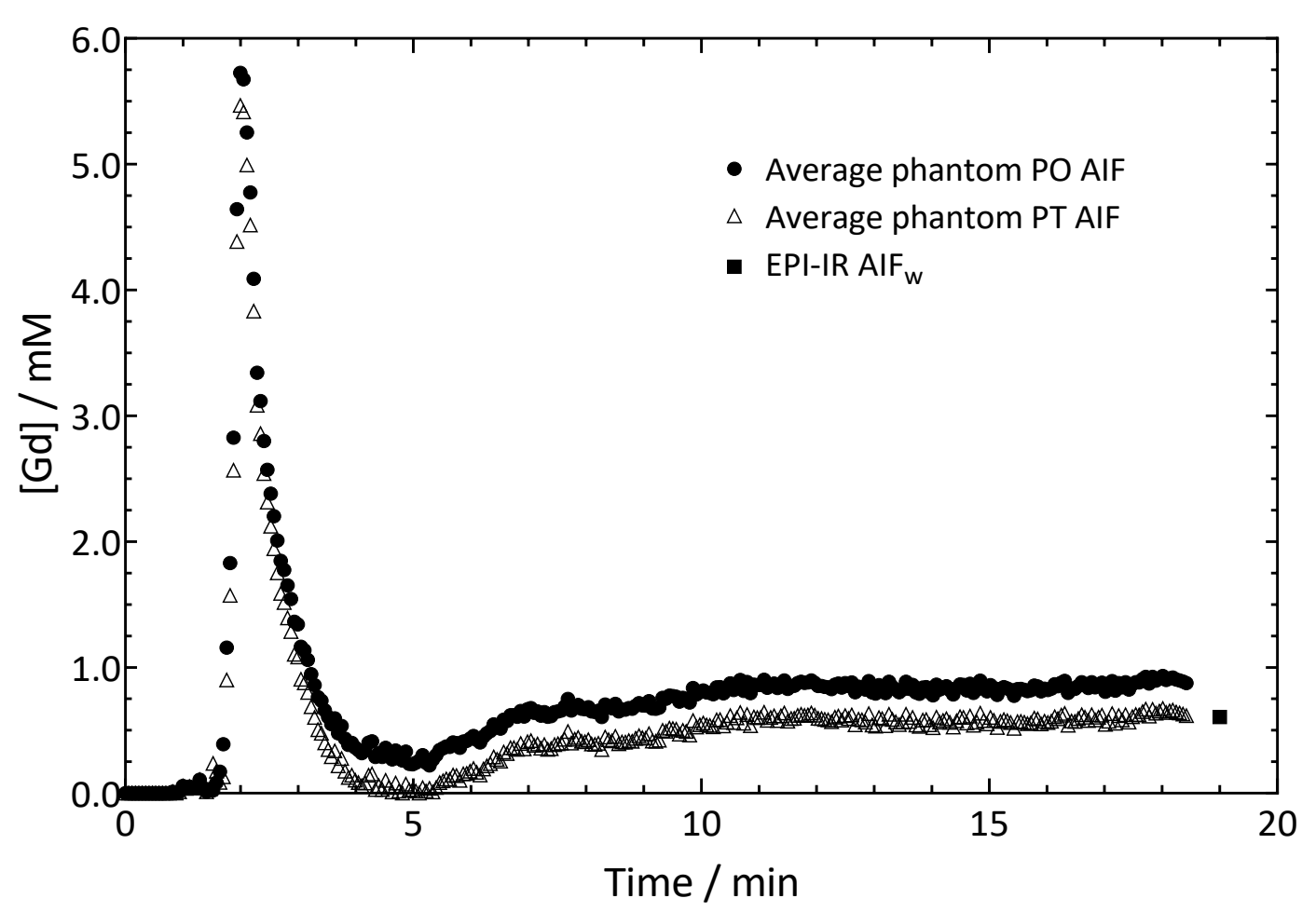

Figure 5.13: The average phantom PO AIF (circles) shown with the newly-formed PT AIF (triangles). The gold-standard AIF washout value determined using EPI-IR (square) was inserted for reference although it was not necessarily measured at the time shown.

MOLLI are accurate and not significantly affected by flowing water.

\subsection{Discussion}

The goal of this chapter was to validate the methods required to create the PT AIF in static and flowing water phantoms before it was applied clinically. It was posited that the PT AIF is more accurate than the PO AIF method because it avoids the large errors in the measurement of the baseline phase that affect the whole PO AIF curve. 


\subsubsection{Static Phantom Data}

IR techniques provide the greatest amount of $T_{1}$ weighting in MR acquisitions. However, their long acquisition times exclude them from being used to measure the AIF in its entirety. They can only be used in regions where the [Gd] is stable, namely the baseline and washout regions of the AIF. With EPI-IR acquisitions, which are considered to be the gold-standard $T_{1}$ measurement method, the MR signal fully relaxes back to its equilibrium value before the following RF inversion pulse. While this time increase is not usually a consideration during phantom imaging, it is a significant concern in clinical imaging where longer scan times can result in patient discomfort, imaging artefacts due to patient movement, and it reduces patient throughput. This was the motive for developing and using faster $T_{1}$ measurement techniques that maintain $T_{1}$ accuracy, such as MOLLI. However, MOLLI was originally created for cardiac acquisitions and has not previously been used for the human brain. The phantom experiments reported here were designed to investigate MOLLI's effectiveness and accuracy when measuring $T_{1}$ in a phantom designed to resemble the SSS.

The $r_{1}$ (and $r_{1}^{*}$ ) of each of the $T_{1}$ (and $T_{1}^{*}$ ) sequences varied significantly (see Table 5.3). These $r_{1}$ and $r_{1}^{*}$ values were obtained only for the data shown in Table 5.2 and did not include data that were removed based on the aforementioned fit requirements (i.e. at least two SI data points must be between $0.5 \mathrm{~T}_{1} \leq \mathrm{TI} \leq 3 \mathrm{~T}_{1}$ ). The MOLLI $r_{1}$ values were significantly lower than the EPI-IR $r_{1}$ and MOLLI $r_{1}^{*}$ fit values. The MOLLI $r_{1}^{*}$ and the MOLLI ${ }_{\text {comb }} r_{1}^{*}$ values were the most similar to the EPI-IR $r_{1}$ value.

It has been reported that a flowing medium can be a considerable source of error 
in $\mathrm{T}_{1}$ and AIF measurements [87], especially in vivo where hematocrit, blood temperature, and blood oxygen levels affect the measurement [88]. It is important to validate that $T_{1}$ can be measured accurately in the dynamic phantom with water flowing. By comparing $T_{1}^{*}$ values for flowing water to those measured without flow, the amount of flow-related effects could be determined in phantom measurements. Unfortunately, this could not also be performed in vivo. Since EPI-IR was not able to be measured in the dynamic phantom, only the effect on MOLLI T1 data was observed. The flow ratio values show that the $T_{1}$ 's measured with MOLLI ${ }_{0}$ and MOLLI ${ }_{1}$ are not significantly affected by flow.

\subsubsection{AlF Data Acquisition}

The dynamic phantom system was used so that the data measured within the phantom resembled an AIF acquisition performed in the human body. While the system had many similarities to an in vivo acquisition (e.g. the diameter of the tubing matched that of the SSS) a few differences exist based on the construction of the phantom system itself. For instance, the tubing length used for the phantom was considerably longer than the length of vasculature in the human body. This extra length is due, in part, to the need for the flow system controls to be in the MR control room, outside of the main MR scanner room, due to the metallic composition of the pump system. Additionally, a large volume reservoir was used to ensure that there would be a sufficient volume of water in the tubing at all times. Any air in the phantom would cause errors in the acquisition of data and cause damage to the pump system itself. These additional setup requirements are part of the reason why the shape of the PO AIF acquired in the dynamic phantom was not 
identical to one obtained clinically. While essentially the same in all the major features, they are a bit different in some ways. A comparison of a phantom AIF acquisition to a clinical AIF will be given in the next chapter (see Fig. 6.3).

MOLLI was expected to be a significantly better method for measuring $T_{1}$ in a flowing medium because it is fast, it has a single inversion, it is relatively free of artefacts, and the $T_{1}$ values obtained have good accuracy and precision [34]. Other methods for measuring $T_{1}$ are available but none of them offer all of these advantages. This work validated that the MOLLI Works-in-Progress package (Siemens AG, Erlangen, Germany) provided the anticipated accuracy and precision for the range of $T_{1}$ values that can be encountered during a clinical AIF study using both a set of static water phantoms and a flowing-water phantom that models an in vivo acquisition. This is primarily shown based on good agreement with EPI-IR. The average AIF $_{\mathrm{w}}$ value measured with MOLLI was $0.59 \pm$ $0.03 \mathrm{mM}$ (from Table 5.4). This is within error of the expected [Gd] in the washout $(0.7 \pm$ $0.1 \mathrm{mM})$ and more accurate than the average $\phi_{\mathrm{w}}$ data $(0.87 \pm 0.05 \mathrm{mM})$. It should be noted that for $[\mathrm{Gd}] \geq 0.8 \mathrm{mM}$, the uncorrected MOLLI values were in better agreement with the EPI-IR values than the corrected ones. This was a surprise and remains unexplained. However, since the agreement between the uncorrected values and EPI-IR is very good, it suggests strongly that these uncorrected values are the true values. Furthermore, for $0.2 \leq[\mathrm{Gd}] \leq 0.8 \mathrm{mM}$, the concentration range where AlF $_{\mathrm{w}}$ is most likely to fall, the difference between the corrected and uncorrected $T_{1}$ values was very small.

To construct the PT AIF, $\mathrm{T}_{1}$ imaging was performed both before and after the acquisition of the PO AIF to determine the change in [Gd]. This resulted in an extra 5 min 


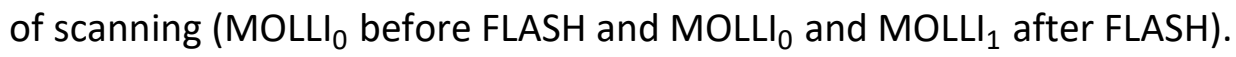

Each of the six acquired PT AIFs was lower when compared to the original PO AIF data (see Table 5.4). These shifts were based on the difference in washout values measured with phase and $\mathrm{T}_{1}$.

\subsection{Conclusions}

This chapter demonstrated the use of phantoms in the formation of the PT AIF method. This procedure involved the careful measurement of $T_{1,0}$ and $T_{1, w}$ using MOLLI sequences as a fast, accurate replacement of IR imaging techniques. This was first done in static phantoms then performed in the dynamic phantom. $T_{1}^{*}$ and $r_{1}^{*}$ of the $[\mathrm{Gd}]$-appropriate MOLLI acquisitions were within the measured values of EPI-IR data with no statistically significant differences observed. The MOLLI $_{\text {comb }}$ calibration curve was created from $\mathrm{MOLLI}_{0}$ and $\mathrm{MOLLI}_{1}$ results since neither sequence was reliable for the whole [Gd] range considered. For $\left[\mathrm{Gd}\right.$ ] measurements in the AIF washout, $\mathrm{MOLLI}_{\text {comb }}$ produced more accurate $[G d]$ values compared to EPI-IR $[G d]$ values $(r=0.995, P<0.0001)$ than the phase provided $(r=0.067, P=0.898)$. Next, FLASH $\phi(t)$ data were measured as the GBCA passed through the input of the phantom system to create the PO AIF. The computation of the full AIF was then performed from the unwrapped $\phi(t)$ data using Eq. 2.109. The accurate AlF $_{\mathrm{w}}$ values from the measured $\mathrm{T}_{1,0}$ and $\mathrm{T}_{1, \mathrm{w}}$ values were computed using Eq. 4.12. Finally, the "correction" of the AIF to replace the larger uncertainties associated with $\phi_{0}$ with the smaller uncertainties from $\phi_{w}$ using Eq. 4.4. (i.e. the PT AIF) was employed. This resulted in a PT AIF that was significantly different than the PO AIF $(P<0.0001)$. In the following 
chapter, this method will be applied clinically to observe the effects that the PT AIF has on the acquisition of quantitative, clinical DCE-MRI parameters.

The predictions of the theoretical work presented in Chapter 3 and the simulations given in Chapter 4 show that the PO AIF can be expected to give [Gd] values that are too high by approximately $0.2 \mathrm{mM}$. A comparison of the $[\mathrm{Gd}]$ values for the washout obtained from the PO AIF and the MOLLI $T_{1}$ measurements shows that this is true for the dynamic phantom. Although a true gold standard AIF was not available for validation of the results presented here, this observations serves to validate that the PT AIF measured in the dynamic phantom is more accurate than the PO AIF. 


\section{Chapter 6}

\section{Clinical DCE-MRI Studies}

\subsection{Introduction}

Dynamic Contrast-Enhanced (DCE)-MRI studies of tumours can provide valuable information about the tumour vascular environment. With the tracer kinetic model used for the work presented here, the extended Tofts-Kety model (see Section 2.2.2), three parameters are obtained: the volume of plasma per unit volume of tissue $\left(v_{p}\right)$, the volume of the extravascular extracellular space (EES) per unit volume of tissue $\left(v_{\mathrm{e}}\right)$, and the volume transfer coefficient $\left(K^{\text {trans }}\right)$ which relates to the permeability of the capillary system. An additional useful parameter is $k_{e p}$, the rate constant for flow from the EES into the vascular space, which is defined by $\mathrm{k}_{\mathrm{ep}} \equiv \mathrm{K}^{\text {trans }} / \mathrm{v}_{\mathrm{e}}$. These parameters have been shown to be useful in the characterization and diagnosis of gliomas $[20,22]$ and a valuable aid for differentiating scar tissue from glioma recurrence following radiation therapy [89].

To get reliable information from DCE-MRI, it is important to have an accurate 
Arterial Input Function (AIF). The conventional method for computing the AIF from MR phase measurements, referred to here as the "Phase-Only" (PO) method, is known to be susceptible to systematic experimental error due to inaccuracy of the baseline phase measurement. This effect was investigated theoretically in Chapter 3 and, as a result of this analysis, a new approach, referred to here as the "Phase and $\mathrm{T}_{1}$ " (PT) method, was proposed in Chapter 4 and validated using computer simulations and experiments with a dynamic phantom in Chapter 5.

An important aspect of the PT AIF method is the incorporation of an accurate and precise $T_{1}$ measurement for both the baseline and the washout sections of the AIF. For the phantom studies, these $T_{1}$ values were obtained using EPI-IR, a sequence which is well known to provide reliable $T_{1}$ measurements. Unfortunately, EPI-IR is too time-consuming for clinical studies. To overcome this issue, the use of a MOLLI sequence was proposed. MOLLI is much faster than EPI-IR and it has been shown to be useful in cardiac MR [34] but it was not clear if it would provide reliable results for the brain. In Chapter 5 it was shown with two different phantom systems that the $T_{1}^{*}$ values measured with MOLLI are in very good agreement with the "gold-standard" EPI-IR $\mathrm{T}_{1}$ values. This very strong agreement of the uncorrected $T_{1}$ relaxation times with the gold-standard values was unexpected. It was anticipated that the $T_{1}{ }^{\prime}$ s (i.e. the corrected values) would be the ones in good agreement with the EPI-IR values. Nevertheless, the goal was to find a timeefficient way of measuring relaxation times that are in good agreement with the EPI-IR T 1 values and the $T_{1}^{*}$ measured with MOLLI provided this for the phantoms used in Chapter 5. 
The reason why the $T_{1}^{*}$ values are apparently more accurate than the $T_{1}$ values is not clear. Since it could be related to properties of the phantoms that may not be present in humans, it was decided that both corrected and uncorrected $\mathrm{T}_{1}$ values would be used for the clinical study discussed in this chapter. Unfortunately, it was not possible to compare the measured $T_{1}^{*}$ and $T_{1}$ values to EPI-IR values with this patient cohort due to clinical time constraints.

The PT AIF method proposed in Chapter 4 was tested using human participants previously diagnosed with high-grade gliomas (HGG). This is the topic of the current chapter. The goals of this work were: 1 ) to determine the feasibility of using the PT AIF method with a patient population; 2 ) investigate the relative merits of using $T_{1}$ versus $T_{1}^{*}$ for the PT AIF method with a cohort of HGG patients; 3 ) to show that the PT AIF is superior to the PO AIF; and 4) to compare the DCE-MRI parameters calculated with the PT AIF with those obtained using the PO AIF.

\subsection{Methods}

\subsubsection{Study Design}

The study was performed on a Siemens 3 T Magnetom Trio (Siemens AG, Erlangen, Germany) with a 32-channel head coil. Patients recruited for this study had histologicallyconfirmed high-grade gliomas (HGG). The study had 3 patients with WHO-classified grade III and 18 patients with grade IV tumours. These participants were selected from a group of 107 patients from an ongoing study. Demographic information for each patient is 
Table 6.1: Patient demographic data.

\begin{tabular}{ccccc}
\hline Patient ID & Age & Sex & Tumour Type & Tumour Grade \\
\hline 11 & 53 & Male & Anaplastic glioma & III \\
15 & 63 & Male & Glioblastoma & IV \\
18 & 38 & Female & Astrocytoma & III \\
19 & 54 & Male & Glioblastoma & IV \\
26 & 76 & Male & Glioblastoma & IV \\
40 & 56 & Male & Glioblastoma & IV \\
41 & 59 & Male & Glioblastoma & IV \\
46 & 62 & Male & Glioblastoma & IV \\
47 & 67 & Female & Glioblastoma & IV \\
48 & 70 & Male & Glioblastoma & IV \\
49 & 70 & Female & Glioblastoma & IV \\
54 & 77 & Male & Glioblastoma & IV \\
56 & 42 & Male & Glioblastoma & IV \\
57 & 57 & Female & Anaplastic oligodendroglioma & III \\
62 & 63 & Female & Astrocytoma & IV \\
63 & 74 & Female & Astrocytoma & IV \\
73 & 44 & Male & Astrocytoma & IV \\
75 & 70 & Male & Astrocytoma & IV \\
78 & 83 & Female & Astrocytoma & IV \\
80 & 48 & Male & Astrocytoma & IV \\
82 & 73 & Female & Astrocytoma & IV \\
\hline
\end{tabular}


provided in Table 6.1. Only 21 patients ( 13 males, 8 females, age range $=38-83$ yrs) received all of the necessary imaging sequences to participate in the research presented in this thesis. These patients had the additional scans added to the standard clinical protocol, after informed consent was obtained. FLASH data were acquired after a dose of $0.05 \mathrm{mmol} \mathrm{kg}^{-1}$ of gadobutrol (Gadovist ${ }^{\circledR} 1.0 \mathrm{M}$, Bayer HealthCare Pharmaceuticals, Toronto, Ontario, Canada) was injected as a bolus with a power injector at a rate of $5 \mathrm{~mL}$ $\mathrm{s}^{-1}$ followed by a saline flush. Patients were part of a dual-injection study, where the first injection was used for this DCE-MRI analysis and the other injection was for a separate study. The main study, including the sub-study discussed in this chapter, was approved by The Ottawa Hospital Research Ethics Board. The research involving human participants was also approved by the Carleton University Research Ethics Board (file no. 105320).

\subsubsection{Data Acquisition}

The additional sequences included for the research were MOdified Look-Locker Inversion Recovery (MOLLI) and 3D Fast Low Angle Shot (FLASH). These were the same sequences with the same parameter settings as were used with the dynamic phantom. For a full list of settings for these sequences see Sections 5.2.2 and 5.2.3.

The 3D FLASH sequence was used to acquire the phase data from which the PO AIF was computed. The sequence was started before the injection of the GBCA was given and repeated until the $[\mathrm{Gd}]$ values had reached equilibrium in the AIF washout. The MOLLI sequences were used to get the necessary $T_{1}$ relaxation values to allow for the conversion of the PO AIF into the PT AIF. 
The GBCA was injected after five repetitions of the 3D FLASH sequence, which took about 10-15 s, and the injection lasted for a few seconds. Unlike for the dynamic phantom, the total scan time for the 3D FLASH acquisition had to be constrained since the scanning was done with human patients. The 3D FLASH sequence took $4.67 \mathrm{~min}$ to run and 80 sets of dynamic data were acquired. In most instances, an equilibrium AIF washout was achieved in this time frame.

The phase values as a function of time were obtained from the phase images using a circular region of interest (ROI) placed wholly within the superior sagittal sinus (SSS) on multiple slices. While the specific ROI size varied from patient to patient, it contained approximately $10-15$ pixels. The phase dataset was then unwrapped $[52,86]$ and used to compute the PO AIF using Eq. 2.109. The SSS was chosen for these phase measurements partly because it usually runs nearly parallel to $\mathbf{B}_{\mathbf{0}}$. When this is the case the geometry factor defined in Eq. 2.110 reduced to 1/3. For each slice, the respective angle between the SSS and $\mathbf{B}_{\mathbf{0}}(\theta)$ was taken into account for each measurement of the phase.

The ROI averaged phase values were obtained from the phase images using Image $^{\circledR}$ (National Institutes of Health, Bethesda, United States). The subsequent calculation were performed with in-house code written by Dr. Gregory Cron using IDL ${ }^{\circledR}$ (ITT Visual Information Solutions, Broomfield, United States).

The PO AIF was evaluated from phase measurements taken from the SSS. Consequently, this function is actually the venous output function (VOF). The differences between the VOF and the AIF, along with the pros and cons of using the VOF as a surrogate for the AIF were discussed in Section 3.2.2. The main advantage is that, since the SSS is 
larger than the cerebral arteries, errors due to partial volume averaging are significantly reduced when the VOF is used. Even though it is acknowledged that the measured function is actually a VOF, it will be referred to as an AIF in this chapter.

The TI values used for the MOLLI $I_{0}$ sequence were chosen to fully cover the $T_{1}$ regrowth curve for the $T_{1}$ of room temperature water (for the phantom study) as well as for the $T_{1}$ of blood (for the clinical study) at $3 \mathrm{~T}$. These values were about $3000 \mathrm{~ms}$ [43] and $2000 \mathrm{~ms}$ [72], respectively. In both cases, 6 of the 8 TI values used were in the range $0.5 \mathrm{~T}_{1} \leq \mathrm{Tl} \leq 3 \mathrm{~T}_{1}$, which covers the most important part of the $\mathrm{T}_{1}$ regrowth curve - the portion where it changes the most.

The MOLLI ${ }_{1}$ sequence was designed for measuring $T_{1, w}$, the $T_{1}$ value for the AIF washout $\left(\mathrm{AlF}_{\mathrm{w}}\right)$; however, the range of $\mathrm{T}_{1, \mathrm{w}}$ values that might occur for a cohort of HGG patients was not well known. It was decided that the protocol should be able to accurately measure the $T_{1, w}$ values for AlF $_{w}$ values in the range $0.1 \mathrm{mM} \leq[\mathrm{Gd}] \leq 1.0 \mathrm{mM}$. Using Eq. 2.84 with $r_{1}=4.1 \mathrm{mM}^{-1} \mathrm{~s}^{-1}$ [43] and $\mathrm{T}_{1,0}=1932 \mathrm{~ms}$ [72], it can be shown that this corresponds to $T_{1, w}$ values between $200 \mathrm{~ms}$ and $1000 \mathrm{~ms}$. The MOLLI ${ }_{1}$ sequence was set up to cover this range of $T_{1}$ values. With the set of TI values chosen for MOLLI 1,6 of the $8 \mathrm{TI}$ values are in the range $0.5 \mathrm{~T}_{1} \leq \mathrm{Tl} \leq 3 \mathrm{~T}_{1}$ for most of the indicated range of $\mathrm{T}_{1}$ values. As an added precaution, $T_{1, w}$ was also measured using $\mathrm{MOLLI}_{0}$, in case the $[\mathrm{Gd}]$ for $\mathrm{AlF}_{\mathrm{w}}$ fell below $0.1 \mathrm{mM}$ for some of the patients in the study. It should be noted that the MOLLI $_{1}$ sequence was only added to the protocol for this study after the measurements for the first five patients had been completed.

When an IR sequence is performed, the signal intensity (SI) data immediately 
following the initial inversion pulse should be negative and then become positive at a time dictated by the $T_{1}$ of the sample. However, the MR scanner only acquires SI data as a positive value. The algorithm used to determine which of the data points should be negative and fit the data to Eq. 2.79 to obtain $A, B$, and $T_{1}^{*}$ values is described in Section 5.2.2.1. The true $T_{1}$ values are then calculated from these $T_{1}^{*}$ values using Eq. 2.80. The $T_{1}^{*}$ and $T_{1}$ values were then averaged over a circular ROI placed over the SSS and over the centre eight slices. The $T_{1}^{*}$ and $T_{1}$ values obtained in this way from the $M O L L I_{0}$ data that were measured before the GBCA injection will be denoted as $T_{1,0}^{*}$ and $T_{1,0}$, respectively.

Before $T_{1, w}^{*}$ and $T_{1, w}$ could be converted to [Gd] using Eq. 2.84, an appropriate longitudinal relaxivity value $\left(r_{1}\right)$ had to be determined. The value measured in the phantom studies could not be directly applied to the clinical acquisitions since $r_{1}$ is dependent on the magnetic field strength $\left(B_{0}\right)$, the specific GBCA used, the type of medium containing the GBCA, and the medium temperature $[43,44]$. For the static phantoms, $r_{1}$ and $r_{1}^{*}$ were calculated from a calibration curve that was created using various [Gd] and measured $T_{1}$ and $T_{1}^{*}$ values, respectively. This cannot be feasibly performed in vivo, as there is no reliable, non-invasive method to determine the [Gd] of the blood since this would involve blood sampling. The $r_{1}^{*}$ value obtained from the roomtemperature water, static phantom measurements described in Chapter 5 (4.98 \pm $\left.0.15 \mathrm{mM}^{-1} \mathrm{~s}^{-1}\right)$ matched the value obtained from Stalder et al. $\left(4.7 \pm 0.2 \mathrm{mM}^{-1} \mathrm{~s}^{-1}\right)[43]$ to within experimental error. Therefore, it is reasonable to assume a similar $r_{1}$ value measured from body-temperature blood at 3 T obtained from the same work can be used for the clinical data obtained in this study $\left(r_{1}=4.1 \pm 0.2 \mathrm{mM}^{-1} \mathrm{~s}^{-1}\right)[43]$. The [Gd] values 
for the washout were calculated for both $T_{1}^{*}$ and $T_{1}$ using Eq. 2.84 with this $r_{1}$ value and the $T_{1,0}^{*}$ and $T_{1,0}$ values measured for that patient with $M O L L_{0}$, respectively.

The PT AIF was constructed from the PO AIF using the theory described in Section 4.2.2 and Eq. 4.4 with the procedure initially described for the dynamic phantom data (Section 5.2.3). PT AIF curves were constructed using both $T_{1, w}^{*}$ and $T_{1, w}$.

DCE-MRI parameter maps for $\mathrm{v}_{\mathrm{p}}, \mathrm{K}^{\text {trans }}, \mathrm{v}_{\mathrm{e}}$, and $\mathrm{k}_{\mathrm{ep}}$ were computed from both the PO AIF and the PT AIF with Olea Sphere 2.2 (Olea Medical, La Ciotat, France). Physiologically impossible values (e.g. $v_{p}, v_{e}>1$ ) and data that appeared to be noise (i.e. small, repetitive values) were removed from each set of parameter maps. Mean parameter values were measured using ROls positioned on the enhancing tumour volumes. The ROls were drawn manually on the parameter maps, often over several slices, by a medical student under the supervision of a board-certified neuroradiologist. Parameters from each AIF method were then compared using a paired $t$-test. An example of the ROI placement is shown in Fig. 6.1.

\subsection{Results}

The primary objectives of this clinical study were to demonstrate that the PT AIF is superior to the PO AIF and to observe the difference between the DCE-MRI parameters calculated with these two AIFs. Since the PT AIF is expected to be more accurate, the corresponding DCE-MRI parameters calculated from it should also be more accurate.

PO AIF data were acquired for each of the 21 patients. Each of the AIFs, save for one, followed the typical shape of an AIF. The exception to this was for patient 41 where 


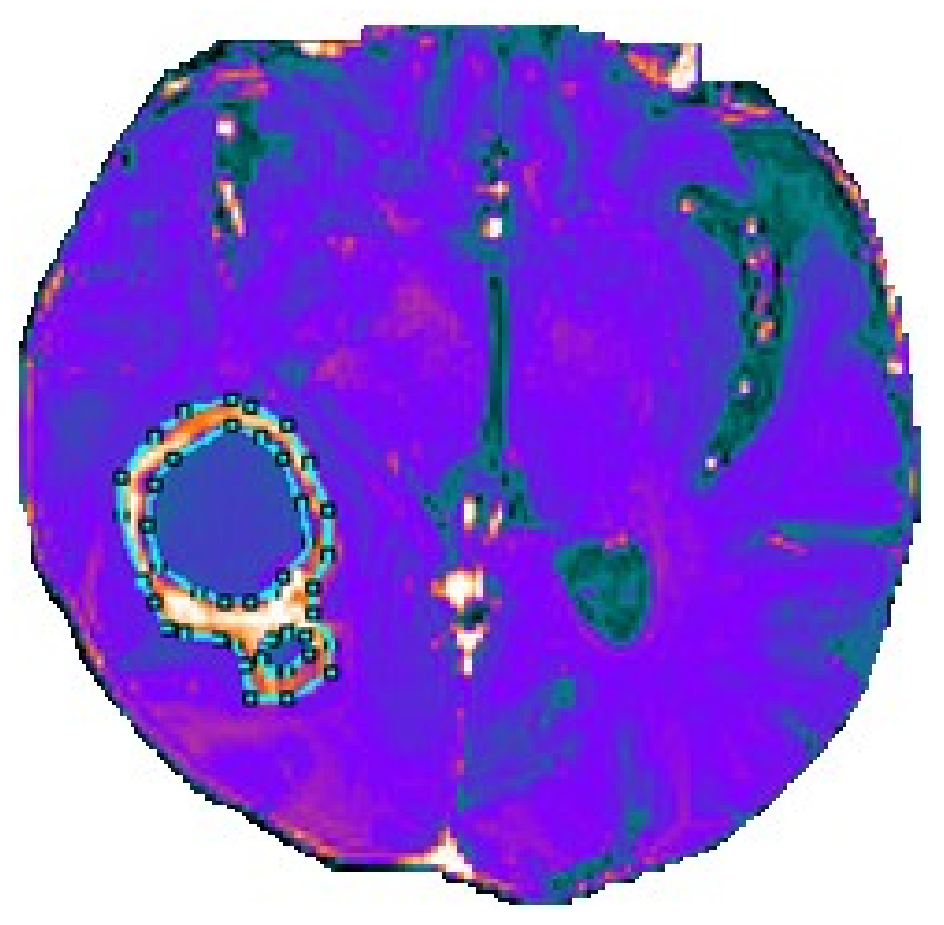

Figure 6.1: Example of an ROI placed on an axial slice over the enhancing tissue volume of an astrocytoma for a $\mathrm{T}_{1}$-weighted anatomical image. Note that the region in between the blue lines of the "figure- 8 " shape (i.e. the enhancing, orange portion) are included in the ROI. 
the GBCA was injected twice during the DCE-MRI acquisition. This was due to complications with the injection, as the peak [Gd] after the first injection was only 2.75 mM. The second injection was more typical of a DCE-MRI acquisition with a peak amplitude of $4.64 \mathrm{mM}$. It was the data from this injection that were used for the subsequent DCE-MRI analysis. Note that the data for patient 41 are included with the results presented in this chapter; however, due to the double injection, these results were excluded from any averages calculated for the group.

The corrected and uncorrected $\mathrm{T}_{1}$ values measured using $\mathrm{MOLLI}_{0}$ before the GBCA injection, $T_{1,0}$ and $T_{1,0}^{*}$, respectively, are given in Table 6.2 for all 21 patients. The percent difference between the average corrected and uncorrected values is $5.2 \%$ with the corrected values being lower for 20 of the 21 patients.

The corrected and uncorrected $\mathrm{T}_{1}$ values measured using $\mathrm{MOLLI}_{0}$ and $\mathrm{MOLLI}_{1}$ for the AIF washout, $T_{1, w}$ and $T_{1, w}^{*}$, respectively, are given in Table 6.3 for all 21 patients. As mentioned in the previous section, $\mathrm{MOLLI}_{0}$ was included in the protocol for measuring $T_{1, w}$ in case there were instances where $[G d]$ was low enough in the washout that $T_{1, w}$ would be greater than about $1000 \mathrm{~ms}$. It can be seen from Table 6.3 that all of the reported $T_{1}$ values are considerably less than this limit. Consequently, the relaxation times measured with $\mathrm{MOLLI}_{1}$ will be considered to be the more reliable numbers and the $\mathrm{MOLLI}_{0}$ values for the washout will not be discussed further.

The average values of $T_{1, w}$ and $T_{1, w}^{*}$ as measured with $\mathrm{MOLLI}_{1}$ differ by only $0.5 \%$ with about half of the corrected values being lower. It can be also be seen from the table that for most patients, the corrected value is equal to the uncorrected value to within the 
accuracy of the reported values. It should also be noted that for patient 41 , the patient who received the second injection, the values for both $T_{1, w}$ and $T_{1, w}^{*}$ as measured with $\mathrm{MOLLI}_{1}$ are lower than for any of the other patients. This is consistent with some residual GBCA from the first injection still being present in the bloodstream.

Values of $[G d]$ for the AIF washout were calculated from the $T_{1}$ values reported in Tables 6.2 and 6.3 and the results are presented, for each patient, in Table 6.4. Equation 4.12 was used for these computations with $r_{1}=4.1 \mathrm{mM}^{-1} \mathrm{~s}^{-1}$ [43]. The [Gd] values computed using corrected and uncorrected $T_{1}$ data will be denoted AIF $_{\mathrm{w}}$ and $\mathrm{AlF}_{\mathrm{w}}^{*}$, respectively. When calculating AlF $_{w}^{*}$, the uncorrected values of both $T_{1,0}$ and $T_{1, w}$ for each patient were used while the corresponding corrected values were used for calculating $\mathrm{AlF}_{\mathrm{W}}$.

$\mathrm{AIF}_{\mathrm{W}}$ and $\mathrm{AIF}_{\mathrm{W}}^{*}$ values are not given in Table 6.4 for the first five patients since $\mathrm{MOLLI}_{1} \mathrm{~T}_{1}$ values were not available for these patients. The average values for $\mathrm{AlF}_{\mathrm{w}}^{*}$ and $\mathrm{AlF}_{\mathrm{w}}$ (excluding results from patient 41 ) are $0.43 \pm 0.03 \mathrm{mM}$ and $0.42 \pm 0.03 \mathrm{mM}$, respectively. The $\mathrm{AIF}_{\mathrm{w}}$ and $\mathrm{AIF}_{\mathrm{w}}^{*}$ values are also shown in Fig. 6.2 along with the washout values for the PO AIFs for each patient. The washout values obtained from the PO AIFs were calculated as the average over the final few data points in the washout where [Gd] equilibrium occurred and was determined to be $0.70 \pm 0.02 \mathrm{mM}$.

The PT AIFs were generated for each patient from the PO AIFs and the AIF ${ }_{\mathrm{w}}$ values given in Table 6.4 following the procedure proposed in Section 4.2. PT AIFs were created for both $\mathrm{AIF}_{\mathrm{w}}^{*}$ and $\mathrm{AIF}_{\mathrm{w}}$ and these will be denoted as $\mathrm{PT} \mathrm{AIF}^{*}$ and PT AIF, respectively. The whole PO AIF was translated by the same amount to a higher or lower [Gd] value until the 
Table 6.2: MOLLI $_{0} \mathrm{~T}_{1,0}^{*}$ and $\mathrm{T}_{1,0}$ data measured in the SSS. The average \pm SD were obtained using error propagation for each patient. Note that patient 41 was not included in the analysis.

\begin{tabular}{ccc}
\hline Patient ID & MOLLI $_{0} T_{1,0}^{*}(\mathrm{~ms})$ & MOLLI $_{0} T_{1,0}(\mathrm{~ms})$ \\
\hline 11 & $1602 \pm 25$ & $1506 \pm 48$ \\
15 & $1788 \pm 5$ & $1678 \pm 64$ \\
18 & $1755 \pm 36$ & $1697 \pm 61$ \\
19 & $1622 \pm 11$ & $1562 \pm 28$ \\
26 & $1691 \pm 34$ & $1601 \pm 14$ \\
40 & $1693 \pm 26$ & $1653 \pm 39$ \\
41 & $1711 \pm 24$ & $1641 \pm 40$ \\
46 & $1821 \pm 41$ & $1640 \pm 44$ \\
47 & $1808 \pm 44$ & $1657 \pm 56$ \\
48 & $1743 \pm 6$ & $1626 \pm 25$ \\
49 & $1604 \pm 39$ & $1614 \pm 65$ \\
54 & $1780 \pm 6$ & $1730 \pm 32$ \\
56 & $1590 \pm 34$ & $1513 \pm 35$ \\
57 & $1734 \pm 57$ & $1691 \pm 34$ \\
62 & $1753 \pm 18$ & $1663 \pm 29$ \\
63 & $1863 \pm 43$ & $1669 \pm 76$ \\
73 & $1612 \pm 17$ & $1541 \pm 21$ \\
75 & $1500 \pm 5$ & $1475 \pm 27$ \\
78 & $1902 \pm 23$ & $1780 \pm 24$ \\
80 & $1601 \pm 32$ & $1560 \pm 42$ \\
82 & $1735 \pm 18$ & $1598 \pm 28$ \\
\hline Average & $1710 \pm 31$ & $1623 \pm 41$ \\
\hline
\end{tabular}


Table 6.3: $\mathrm{MOLLI}_{0}$ and $\mathrm{MOLLI}_{1} \mathrm{~T}_{1, \mathrm{w}}^{*}$ and $\mathrm{T}_{1, \mathrm{w}}$ data measured in the SSS. The average $\pm \mathrm{SD}$ were obtained using error propagation for each patient. Note that patient 41 was not included in the analysis.

\begin{tabular}{ccccc}
\hline $\begin{array}{c}\text { Patient } \\
\text { ID }\end{array}$ & $\begin{array}{c}\mathrm{MOLLI}_{0} \mathrm{~T}_{1, \mathrm{w}}^{*} \\
(\mathrm{~ms})\end{array}$ & $\begin{array}{c}\mathrm{MOLI}_{0} \mathrm{~T}_{1, \mathrm{w}} \\
(\mathrm{ms})\end{array}$ & $\begin{array}{c}\mathrm{MOLLI} \mathrm{T}_{1, \mathrm{w}}^{*} \\
(\mathrm{~ms})\end{array}$ & $\begin{array}{c}\mathrm{MOLL}_{1} \mathrm{~T}_{1, \mathrm{w}} \\
(\mathrm{ms})\end{array}$ \\
\hline 11 & $647 \pm 47$ & $638 \pm 36$ & $\mathrm{~N} / \mathrm{A}$ & $\mathrm{N} / \mathrm{A}$ \\
15 & $561 \pm 16$ & $594 \pm 24$ & $\mathrm{~N} / \mathrm{A}$ & $\mathrm{N} / \mathrm{A}$ \\
18 & $584 \pm 21$ & $616 \pm 34$ & $\mathrm{~N} / \mathrm{A}$ & $\mathrm{N} / \mathrm{A}$ \\
19 & $728 \pm 15$ & $711 \pm 24$ & $\mathrm{~N} / \mathrm{A}$ & $\mathrm{N} / \mathrm{A}$ \\
26 & $597 \pm 6$ & $618 \pm 19$ & $\mathrm{~N} / \mathrm{A}$ & $\mathrm{N} / \mathrm{A}$ \\
40 & $564 \pm 9$ & $590 \pm 17$ & $496 \pm 11$ & $482 \pm 7$ \\
41 & $329 \pm 14$ & $363 \pm 21$ & $266 \pm 10$ & $295 \pm 7$ \\
46 & $644 \pm 22$ & $682 \pm 28$ & $498 \pm 56$ & $498 \pm 14$ \\
47 & $532 \pm 10$ & $519 \pm 32$ & $465 \pm 17$ & $453 \pm 12$ \\
48 & $609 \pm 1$ & $624 \pm 12$ & $510 \pm 6$ & $508 \pm 9$ \\
49 & $526 \pm 8$ & $558 \pm 65$ & $432 \pm 5$ & $450 \pm 8$ \\
54 & $487 \pm 11$ & $527 \pm 31$ & $385 \pm 11$ & $408 \pm 12$ \\
56 & $627 \pm 6$ & $619 \pm 17$ & $535 \pm 9$ & $501 \pm 8$ \\
57 & $430 \pm 7$ & $443 \pm 21$ & $358 \pm 8$ & $372 \pm 10$ \\
62 & $324 \pm 15$ & $370 \pm 27$ & $272 \pm 8$ & $305 \pm 12$ \\
63 & $503 \pm 18$ & $501 \pm 54$ & $394 \pm 14$ & $410 \pm 22$ \\
73 & $794 \pm 3$ & $827 \pm 31$ & $696 \pm 31$ & $579 \pm 5$ \\
75 & $567 \pm 2$ & $559 \pm 35$ & $483 \pm 18$ & $469 \pm 14$ \\
78 & $463 \pm 7$ & $486 \pm 21$ & $379 \pm 7$ & $402 \pm 8$ \\
80 & $476 \pm 11$ & $480 \pm 21$ & $406 \pm 11$ & $402 \pm 7$ \\
82 & $389 \pm 12$ & $398 \pm 10$ & $341 \pm 5$ & $353 \pm 5$ \\
\hline Average & $553 \pm 16$ & $568 \pm 31$ & $442 \pm 20$ & $440 \pm 11$ \\
& & & &
\end{tabular}


Table 6.4: $\mathrm{AlF}_{\mathrm{w}}^{*}$ and $\mathrm{AIF}_{\mathrm{w}}$ data and average \pm SD obtained using error propagation for each patient. Note that patient 41 was not included in the analysis.

\begin{tabular}{ccc}
\hline Patient ID & AIF $_{\mathrm{w}}^{*}(\mathrm{mM})$ & AIF $_{\mathrm{w}}(\mathrm{mM})$ \\
\hline 11 & $\mathrm{~N} / \mathrm{A}$ & $\mathrm{N} / \mathrm{A}$ \\
15 & $\mathrm{~N} / \mathrm{A}$ & $\mathrm{N} / \mathrm{A}$ \\
18 & $\mathrm{~N} / \mathrm{A}$ & $\mathrm{N} / \mathrm{A}$ \\
19 & $\mathrm{~N} / \mathrm{A}$ & $\mathrm{N} / \mathrm{A}$ \\
26 & $\mathrm{~N} / \mathrm{A}$ & $0.36 \pm 0.02$ \\
40 & $0.35 \pm 0.02$ & $0.68 \pm 0.04$ \\
41 & $0.77 \pm 0.05$ & $0.34 \pm 0.02$ \\
46 & $0.36 \pm 0.06$ & $0.39 \pm 0.02$ \\
47 & $0.39 \pm 0.03$ & $0.33 \pm 0.02$ \\
48 & $0.34 \pm 0.02$ & $0.39 \pm 0.02$ \\
49 & $0.41 \pm 0.02$ & $0.46 \pm 0.03$ \\
54 & $0.50 \pm 0.03$ & $0.33 \pm 0.02$ \\
56 & $0.30 \pm 0.02$ & $0.51 \pm 0.03$ \\
57 & $0.54 \pm 0.03$ & $0.65 \pm 0.05$ \\
62 & $0.76 \pm 0.05$ & $0.45 \pm 0.04$ \\
63 & $0.49 \pm 0.03$ & $0.26 \pm 0.01$ \\
73 & $0.20 \pm 0.02$ & $0.36 \pm 0.02$ \\
75 & $0.34 \pm 0.03$ & $0.47 \pm 0.03$ \\
78 & $0.52 \pm 0.03$ & $0.45 \pm 0.02$ \\
80 & $0.45 \pm 0.03$ & $0.54 \pm 0.03$ \\
82 & $0.58 \pm 0.03$ & $0.42 \pm 0.03$ \\
\hline Average & $0.43 \pm 0.03$ &
\end{tabular}




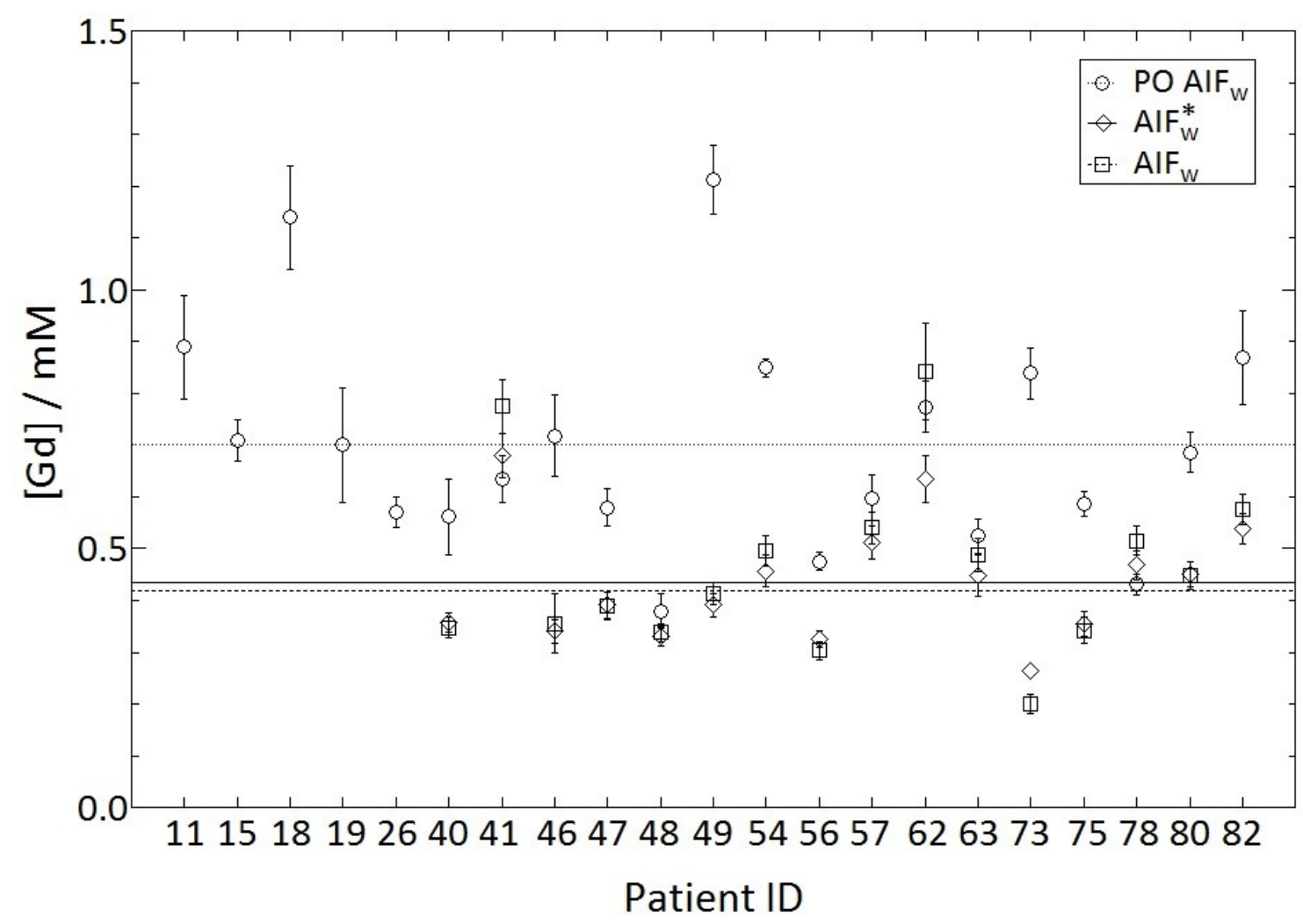

Figure 6.2: The AIF $_{w}$ for the PO AIF (circles) for each patient as well as AIF ${ }_{w}^{*}$ measured with $\mathrm{T}_{1, \mathrm{w}}^{*}$ (diamonds) and $\mathrm{AlF}_{\mathrm{w}}$ measured with $\mathrm{T}_{1, \mathrm{w}}$ (squares). The horizontal dotted, solid, and dashed lines represent the average values of the PO AIF washout, AIF ${ }_{w}^{*}$, and AlF $_{w}$ values, respectively.

washout of the translated curve matched the $\mathrm{AlF}_{\mathrm{w}}$ or $\mathrm{AlF}_{\mathrm{w}}^{*}$ value, depending on which case was being considered. An example comparison of a PO AIF with the corresponding PT AIF is shown for patient 54 in Fig. 6.3. The population-averaged AIF [67] introduced in Chapter 4 is also displayed for comparison.

In Table 6.5, the washout values for the PO AIFs are given for each patient along with the difference between these values and the $\operatorname{AIF}_{\mathrm{w}}^{*}$ and $\mathrm{AIF}_{\mathrm{w}}$ values, these differences being the amount the function is shifted going from the PO AIF to the PT AIF or the PT 


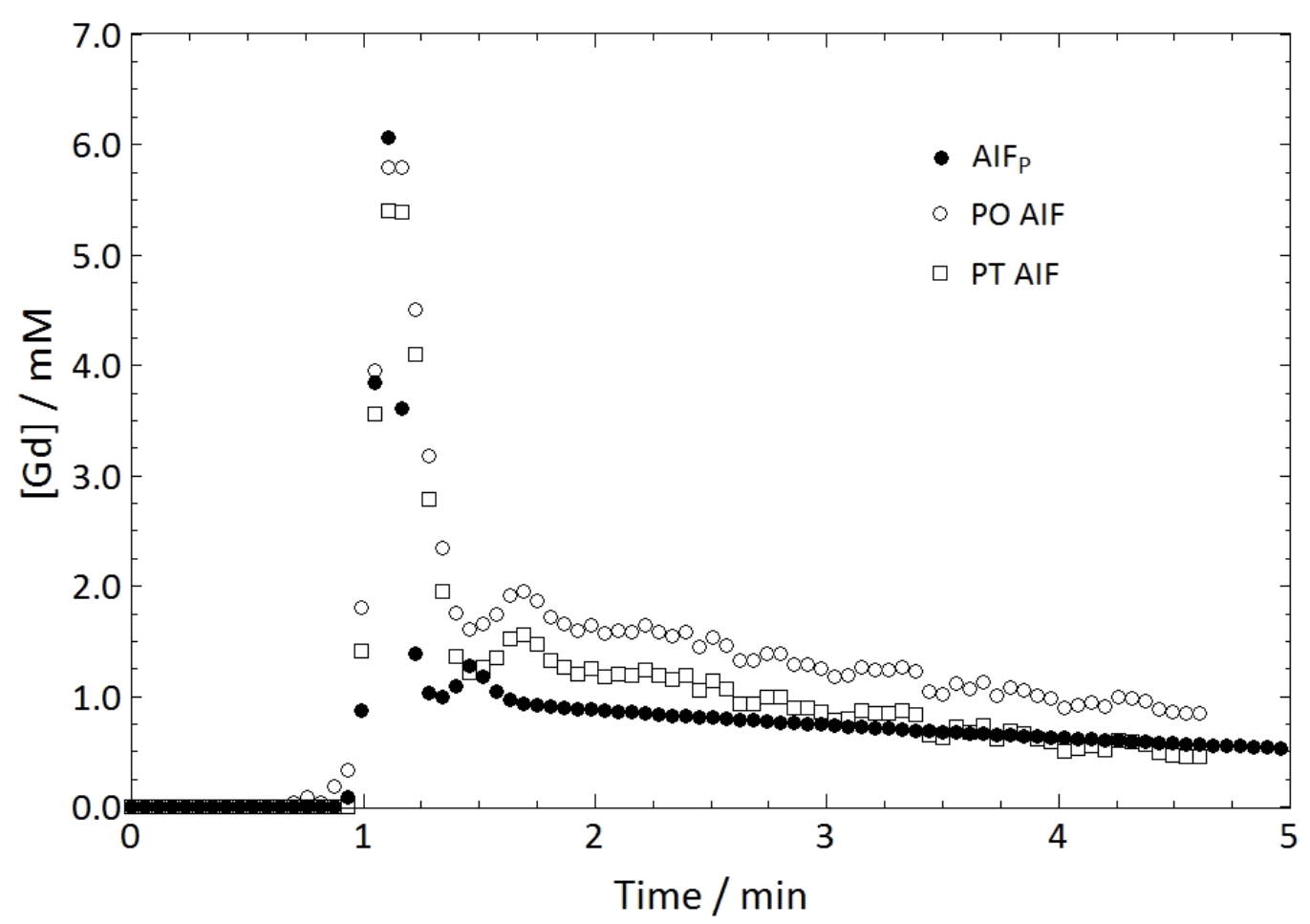

Figure 6.3: An example of a PO AIF and PT AIF acquired for patient 54 compared to a literature AIF (AIF $)$ [67]. AIF was shifted so that its peak value matched, in time, the peak from the PO and PT AIFs.

AIF* $^{*}$ The shift was to lower [Gd] values in all but two cases (patients 41 and 78 ). The range of shifts for PT AlF* was $0.14 \mathrm{mM}$ to $-0.80 \mathrm{mM}$ with a mean \pm SD of $-0.24 \pm 0.24 \mathrm{mM}$. For the PT AIFs, the range was $0.05 \mathrm{mM}$ to $-0.82 \mathrm{mM}$ and the mean \pm SD was $-0.25 \pm 0.22$ $\mathrm{mM}$.

With data from the AIF methods acquired, the resulting quantitative DCE-MRI parameters were computed. The average parameters for the PO and both PT AIF methods are plotted in Fig. 6.4 with the values displayed in Table 6.6. All parameters calculated with the PO AIF demonstrated a statistically significant difference from the PT AIF parameters with a paired t-test (all with $P<0.0001$ ). The average $v_{p}$, 
Table 6.5: Difference in PT AIF $F_{w}^{*}$ and PT AlF $F_{w}$ values compared to the PO AlF $_{w}$ value for each patient. The average value does not include patient 41.

\begin{tabular}{|c|c|c|c|}
\hline Patient ID & PO AIF $F_{w}(m M)$ & $\begin{array}{c}\text { PT AIF }{ }_{w}^{*}-\text { PO AIF } \\
(m M)\end{array}$ & $\begin{array}{c}\text { PT AIF }{ }_{w}-\text { PO AIF } \\
(m M)\end{array}$ \\
\hline 11 & $0.89 \pm 0.10$ & $\mathrm{~N} / \mathrm{A}$ & $\mathrm{N} / \mathrm{A}$ \\
\hline 15 & $0.71 \pm 0.04$ & $\mathrm{~N} / \mathrm{A}$ & $\mathrm{N} / \mathrm{A}$ \\
\hline 18 & $1.14 \pm 0.10$ & $\mathrm{~N} / \mathrm{A}$ & $\mathrm{N} / \mathrm{A}$ \\
\hline 19 & $0.70 \pm 0.11$ & $\mathrm{~N} / \mathrm{A}$ & $\mathrm{N} / \mathrm{A}$ \\
\hline 26 & $0.57 \pm 0.03$ & N/A & $\mathrm{N} / \mathrm{A}$ \\
\hline 40 & $0.56 \pm 0.07$ & -0.21 & -0.20 \\
\hline 41 & $0.63 \pm 0.04$ & 0.14 & 0.05 \\
\hline 46 & $0.72 \pm 0.04$ & -0.36 & -0.38 \\
\hline 47 & $0.58 \pm 0.04$ & -0.19 & -0.19 \\
\hline 48 & $0.38 \pm 0.03$ & -0.04 & -0.05 \\
\hline 49 & $1.21 \pm 0.07$ & -0.80 & -0.82 \\
\hline 54 & $0.85 \pm 0.01$ & -0.35 & -0.39 \\
\hline 56 & $0.48 \pm 0.01$ & -0.18 & -0.15 \\
\hline 57 & $0.60 \pm 0.04$ & -0.06 & -0.09 \\
\hline 62 & $0.77 \pm 0.05$ & -0.01 & -0.12 \\
\hline 63 & $0.52 \pm 0.03$ & -0.03 & -0.07 \\
\hline 73 & $0.84 \pm 0.05$ & -0.64 & -0.58 \\
\hline 75 & $0.59 \pm 0.02$ & -0.25 & -0.23 \\
\hline 78 & $0.43 \pm 0.02$ & 0.09 & 0.04 \\
\hline 80 & $0.69 \pm 0.04$ & -0.24 & -0.24 \\
\hline 82 & $0.87 \pm 0.09$ & -0.29 & -0.33 \\
\hline Average & $0.70 \pm 0.02$ & $-0.24 \pm 0.24$ & $-0.25 \pm 0.22$ \\
\hline
\end{tabular}




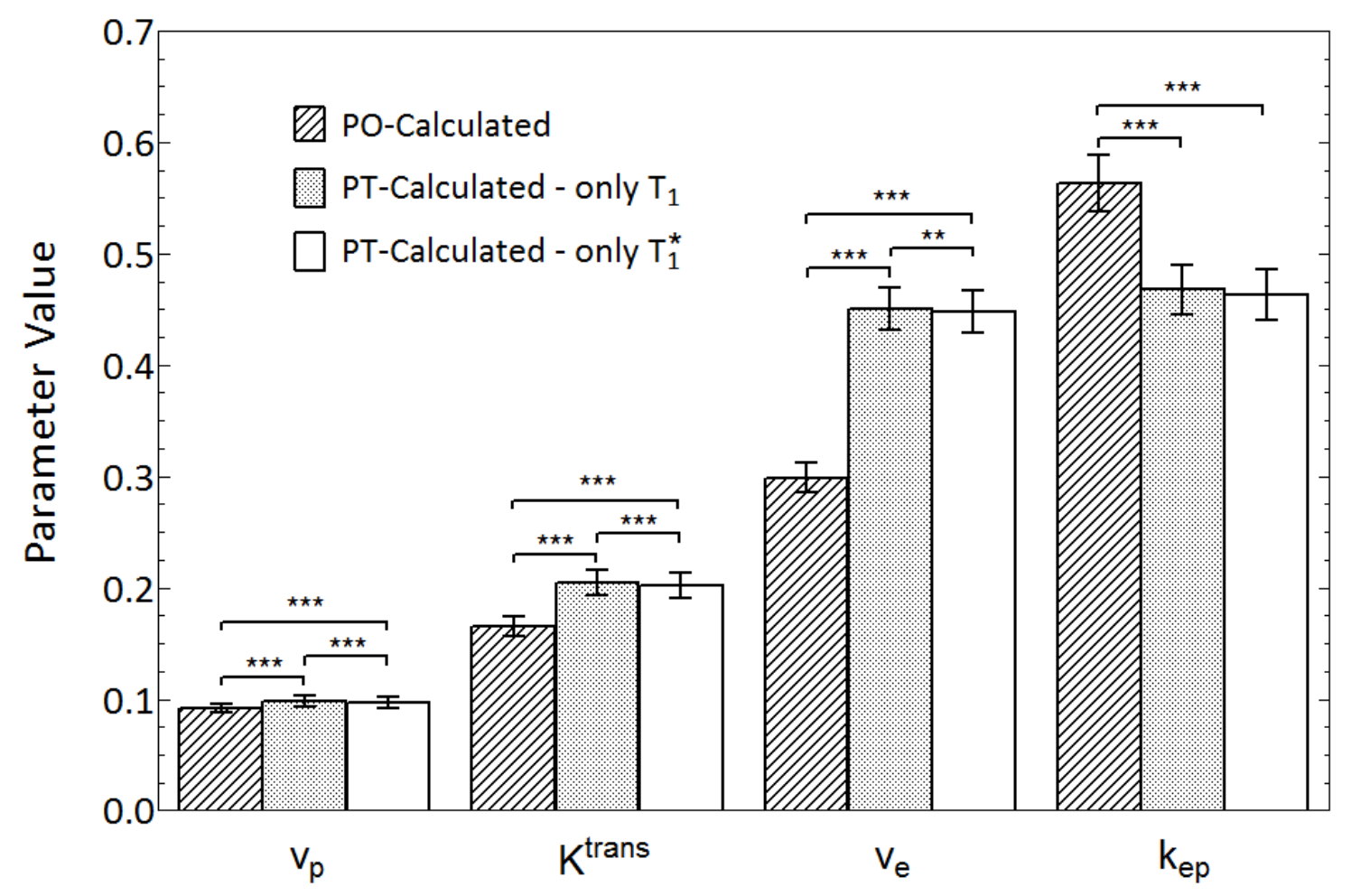

Figure 6.4: DCE-MRI parameters for enhancing tumour tissue computed using the two AIF methods for 21 patients with HGG. A significant difference between the two techniques was observed for each DCE-MRI parameter (***indicates $P \leq 0.001$ and $* * P \leq 0.01$ ). Error bars represent the standard error of the mean. Parameter units are in $\%$ for $v_{p}, v_{e}$ and $\min ^{-1}$ for $\mathrm{K}^{\text {trans }}, \mathrm{k}_{\mathrm{ep}}$. 
Table 6.6: DCE-MRI parameter values from the enhancing volumes for HGGs of grade III $(n=3)$, grade IV $(n=18)$, and grades III and IV together. The final column indicates the percent difference between the PO AIF values and (A) PT AIF or $(B)$ PT AIF $_{w}^{*}$ values. Data are listed as mean \pm standard deviation (coefficient of variation).

(A)

\begin{tabular}{ccccc}
\hline Parameter & Grade & PO AIF Value & PT AIF Value & Difference (\%) \\
\hline \multirow{2}{*}{$\mathrm{v}_{\mathrm{p}}$} & III & $0.058 \pm 0.025(43.1)$ & $0.053 \pm 0.041(77.4)$ & 9.0 \\
$(\%)$ & IV & $0.096 \pm 0.042(43.8)$ & $0.103 \pm 0.052(50.5)$ & 7.0 \\
& III \& IV & $0.092 \pm 0.048(52.2)$ & $0.100 \pm 0.052(52.0)$ & 8.3 \\
\hline $\mathrm{K}^{\text {trans }}$ & III & $0.093 \pm 0.049(52.7)$ & $0.136 \pm 0.076(55.9)$ & 37.6 \\
$\left(\mathrm{~min}^{-1}\right)$ & IV & $0.175 \pm 0.110(62.9)$ & $0.194 \pm 0.115(59.3)$ & 10.3 \\
\hline & III \& IV & $0.165 \pm 0.108(65.5)$ & $0.191 \pm 0.113(59.2)$ & 14.6 \\
\hline $\mathrm{v}_{\mathrm{e}}$ & III & $0.122 \pm 0.080(65.6)$ & $0.375 \pm 0.210(56.0)$ & 101.8 \\
$(\%)$ & IV & $0.336 \pm 0.172(51.2)$ & $0.461 \pm 0.190(41.2)$ & 31.4 \\
\hline & III \& IV & $0.322 \pm 0.182(56.5)$ & $0.456 \pm 0.191(41.9)$ & 34.4 \\
\hline $\mathrm{k}_{\mathrm{ep}}$ & III & $0.952 \pm 0.607(63.8)$ & $0.363 \pm 0.200(55.1)$ & 89.6 \\
$\left(\mathrm{~min}^{-1}\right)$ & IV & $0.502 \pm 0.220(43.8)$ & $0.422 \pm 0.190(41.2)$ & 17.3 \\
& III \& IV & $0.563 \pm 0.319(56.7)$ & $0.419 \pm 0.222(53.0)$ & 29.3 \\
\hline
\end{tabular}

(B)

\begin{tabular}{ccccc}
\hline Parameter & Grade & PO AIF Value & PT AIF ${ }_{\mathrm{w}}^{*}$ Value & Difference (\%) \\
\hline \multirow{2}{*}{$\mathrm{v}_{\mathrm{p}}$} & III & $0.058 \pm 0.025(43.1)$ & $0.053 \pm 0.036(67.9)$ & 9.0 \\
$(\%)$ & IV & $0.096 \pm 0.042(43.8)$ & $0.102 \pm 0.052(51.0)$ & 6.1 \\
& III \& IV & $0.092 \pm 0.048(52.2)$ & $0.099 \pm 0.053(53.5)$ & 7.3 \\
\hline & III & $0.093 \pm 0.049(52.7)$ & $0.138 \pm 0.070(50.7)$ & 39.0 \\
$\mathrm{~K}^{\text {trans }}$ & IV & $0.175 \pm 0.110(62.9)$ & $0.192 \pm 0.113(58.9)$ & 9.3 \\
$\left(\mathrm{~min}^{-1}\right)$ & III \& IV & $0.165 \pm 0.108(65.5)$ & $0.189 \pm 0.112(59.3)$ & 13.6 \\
\hline & III & $0.122 \pm 0.080(65.6)$ & $0.376 \pm 0.221(58.8)$ & 102.0 \\
$\mathrm{v}_{\mathrm{e}}$ & IV & $0.336 \pm 0.172(51.2)$ & $0.459 \pm 0.191(41.6)$ & 30.9 \\
$(\%)$ & III \& IV & $0.322 \pm 0.182(56.5)$ & $0.454 \pm 0.192(42.3)$ & 34.0 \\
\hline & III & $0.952 \pm 0.607(63.8)$ & $0.366 \pm 0.225(61.5)$ & 88.9 \\
$\mathrm{k}_{\mathrm{ep}}$ & IV & $0.502 \pm 0.220(43.8)$ & $0.419 \pm 0.229(54.7)$ & 18.0 \\
$\left(\mathrm{~min}^{-1}\right)$ & III \& IV & $0.563 \pm 0.319(56.7)$ & $0.416 \pm 0.227(54.6)$ & 30.0 \\
\hline
\end{tabular}


$\mathrm{K}^{\text {trans }}$, and $\mathrm{v}_{\mathrm{e}}$ values obtained from both PT AIF methods were higher when compared to the values obtained from the PO AIF for the Grade III \& IV group. Alternatively, the $k_{\text {ep }}$ values decreased from the PT AIF parameters for this group. Additionally, the difference between the parameters obtained from the PO AIF and PT AIF methods are shown in Figs. 6.5 and 6.6 for each patient.

\subsection{Discussion}

It is well known that microvasculature blood flow in tumours is significantly different from that found in healthy tissue. DCE-MRI has been used in an attempt to exploit this property and, while results have been promising, it has not shown the anticipated level of diagnostic and clinically prognostic value. The measurement of accurate perfusion parameters with MRI in a time-efficient manner is challenging. Obtaining a reliable AIF, an essential prerequisite for DCE-MRI analysis, is one of the challenges. The accuracy of $v_{p}, K^{\text {trans }}, v_{e}$, and $k_{e p}$ values obtained from DCE-MRI depends directly on how reliably the AIF can be measured $[20,22]$. This chapter introduced a clinically-viable method to accurately determine the AIF in the human brain. Furthermore, the AIF is acquired in such a manner that makes it feasible to measure a patient's individual AIF rather than resorting to the use of a population-averaged AIF. The in vivo procedure involved: 1) the careful measurement of $\mathrm{T}_{1,0}$ and $\mathrm{T}_{1, \mathrm{w}}$ using MOLLI; 2 ) the measurement of FLASH $\phi(t)$ data as the GBCA passes through the SSS; 3 ) computation of the full AIF from the $\phi(t)$ data using Eq.

2.109 (i.e. the PO AIF); 4) the calculation of an accurate AIF $_{w}$ measurement from the larger uncertainties associated with $\phi_{0}$ with the smaller uncertainties from the measured 

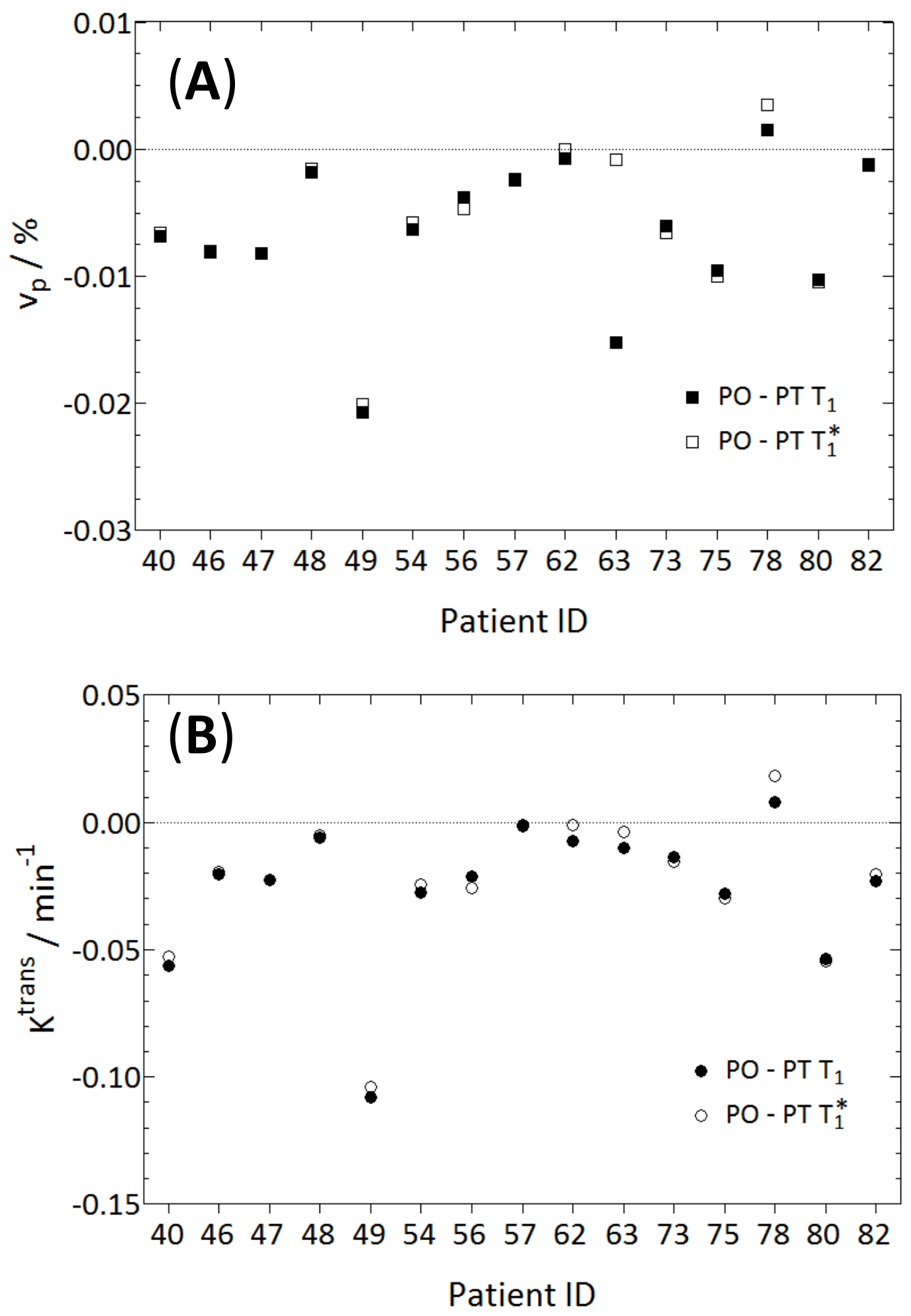

Figure 6.5: Parameter differences between the PO and both PT AIF methods for each patient for $(\mathbf{A}) \mathrm{v}_{\mathrm{p}}$ and $(\mathbf{B}) \mathrm{K}^{\mathrm{trans}}$. 

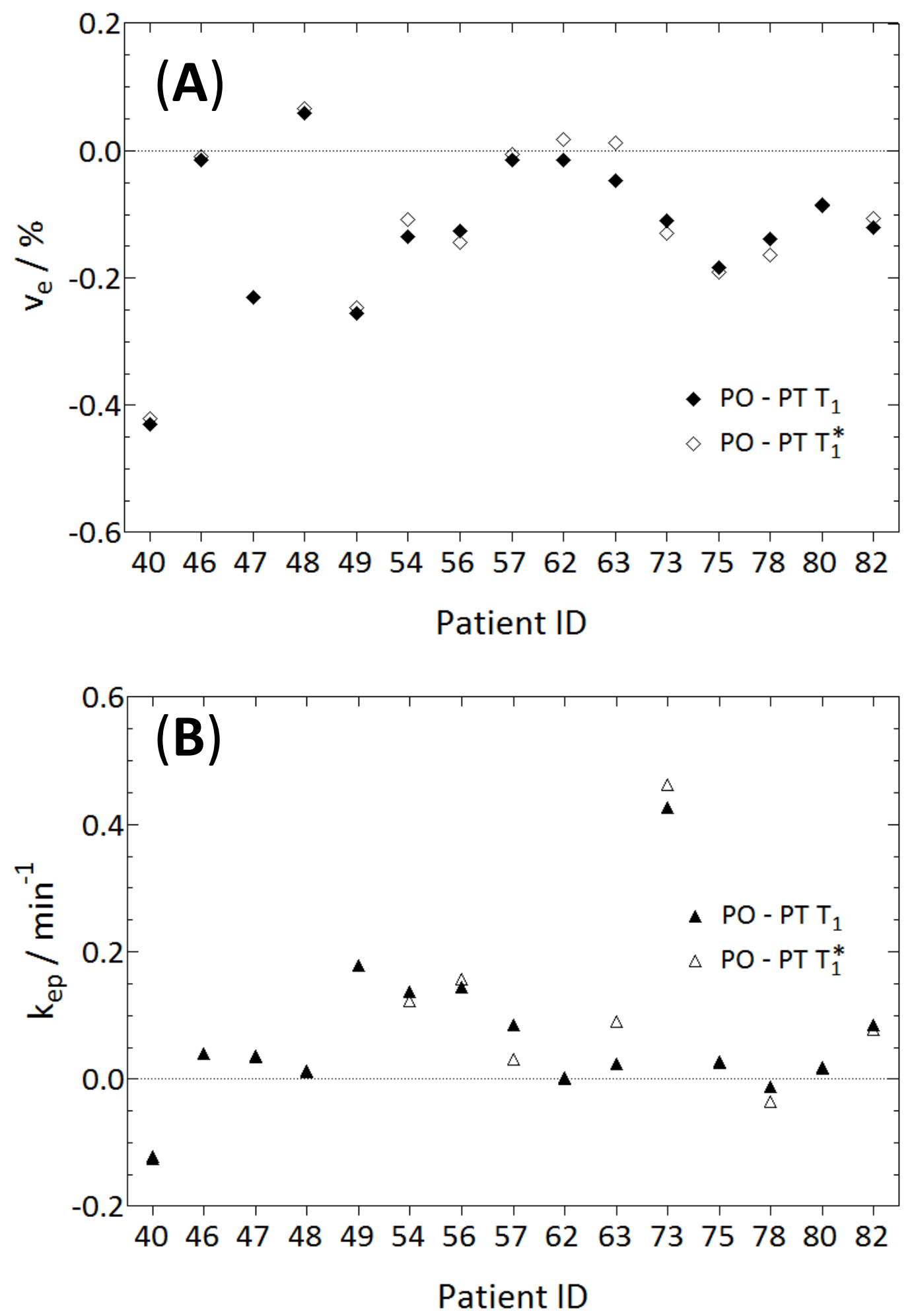

Figure 6.6: Parameter differences between the PO and both PT AIF methods for each patient for (A) $v_{\mathrm{e}}$ and (B) $k_{\mathrm{ep}}$. 
$\mathrm{T}_{1,0}$ and $\mathrm{T}_{1, \mathrm{w}}$ values using Eq. 4.12; and 5) "correcting" the PO AIF to replace washout phase $\left(\phi_{\mathrm{w}}\right)$ using Eq. 4.4 (i.e. the PT AIF).

\subsection{1 $T_{1}$ Measurements}

The $T_{1}$ data used for [Gd] computations in vivo were both the corrected $T_{1}$ and uncorrected $\mathrm{T}_{1}^{*}$ data. $\mathrm{MOLLI}_{0}$ and $\mathrm{MOLL}_{1}$ measurements were performed in a manner similarly done with the dynamic phantom acquisitions presented in Chapter 5. While it was originally designed for cardiac acquisitions, this is the first time that a MOLLI sequence has been used in the human brain for DCE-MRI. From the dynamic phantom data, MOLLI $[G d]$ data matched the $[G d]$ values obtained with the gold-standard $T_{1}$ method of EPI-IR. This helped demonstrate that the PT AIF was reliable, especially for the washout [Gd] values.

This comparison was not able to be performed in the clinical setting as EPI-IR has a long acquisition time $(6.5 \mathrm{~min})$ and is known to have significant artefacts when performed in vivo.. There is currently no other ground-truth method that can be used with human participants to determine $T_{1}$ or $[G d]$ in blood without the extraction of blood from the patient. This is viewed as an unnecessary extreme in most cases, as it is inconvenient and potentially painful for the patient. The accuracy of the dynamic phantom data suggests that MOLLI data are equally accurate in vivo. The MOLLI $T_{1,0}$ data matched the expected $\mathrm{T}_{1}$ range of blood [70,85]. Additionally, the $\mathrm{AlF}_{\mathrm{w}}$ data measured from all three methods in Table 6.4 were lower than the $\mathrm{AlF}_{\mathrm{w}}$ values obtained from the dynamic phantom. 
Theory predicts that the relaxation times measured with a Look-Locker sequence (e.g. MOLLI) need to have a correction factor applied to obtain the true $\mathrm{T}_{1}$ relaxation time values. In Chapter 4, uncorrected MOLLI $\mathrm{T}_{1}$ 's were in very strong agreement with EPI-IR results for the phantoms studied there whereas the "corrected" values were not in good agreement. Consequently, it was decided that both the corrected and the uncorrected $\mathrm{T}_{1}$ data would be used in the clinical trial presented in this chapter to see if one performed better than the other. Overall, the results with these two relaxation times were consistently so similar that it appears, from the data presented here, that whether or not the correction is used has very little effect on the results.

\subsubsection{AIF Measurements}

With the approach presented here, an adjustment of the AIF occurs as a direct result of the removal of the experimental error associated with $\phi_{0}$ and replacing it with the smaller experimental error associated with $\phi_{\mathrm{w}}$. This correction was not introduced in an arbitrary fashion but is a natural consequence of the procedure. The technique uses an additive shift of the non-zero AIF values in the [Gd] direction rather than a multiplicative scaling factor. Nonetheless, the area under the curve (AUC) significantly decreased between the two AIF methods as a result of this shift $(P=0.0004)$. While the effect of altering the AIF using a scaling factor has been reported previously $[74,75,90,91]$, a direct comparison of parameters before and after AIF correction in a human brain population has not been previously reported.

Since the time taken to acquire the AIF was finite and the fact that there will be a 
continuous decrease of a GBCA in vivo, true [Gd] equilibrium could not be achieved. Therefore, the choice of the AIF washout region for each acquired PO AIF was not consistent. While several PO AIFs were relatively stable in the washout, some were steadily decreasing until the end of the acquisition. For situations where the AIF clearly did not reach an equilibrium, the last few $(\sim 2-3)$ data points were used to extrapolate the [Gd] using a linear fit to determine the $[G d]$ at the time $M O L L I_{1}$ was performed. This was only used on a few patients to provide appropriate [Gd] values that took into account the small decrease in the $[\mathrm{Gd}]$ in the washout.

\subsubsection{DCE-MRI Parameters}

Other studies have recognized that inaccuracies in the measurement of the AIF may lead to unreliable DCE-MRI parameter values. To compensate, some groups have suggested applying a multiplicative scaling factor to the AIF $[74,90,91]$. However, the justification for introducing this scaling factor is often unclear. Furthermore, in most of these studies the AIF was not derived from the signal phase, making a direct comparison with the work presented in this thesis difficult. The use of the PT AIF can be thought of as an additive correction that maintains the shape of the AIF, whereas the multiplicative scaling factor has a more pronounced effect on larger values than it does on smaller ones, causing the peak to be relatively more attenuated.

One simulation study showed that a multiplicative scaling factor of 0.80 increased $v_{p}, K^{\text {trans }}$, and $v_{e}$ while having no effect on $k_{e p}[92]$. The results presented in Table 6.5 show a similar trend for the first three parameters, while having a decreased $\mathrm{k}_{\mathrm{ep}}$. Whether 
$k_{e p}$ increases or decreases depends on the relative changes of $K^{\text {trans }}$ and $v_{e}$. However, when these changes are due to a multiplicative scaling factor, it is not surprising that the effect cancels out, leaving $k_{e p}$ unchanged. This could be due, in part, to the large error associated with $\mathrm{v}_{\mathrm{e}}$ as it varied the most of any of the three parameters measured.

For tumours of grade III, grade IV, and grades III and IV together, it was observed that the parameters $\mathrm{v}_{\mathrm{p}}, \mathrm{K}^{\text {trans }}$, and $\mathrm{v}_{\mathrm{e}}$ computed using both PT AIF methods increased significantly relative to those computed from the PO AIF, while $k_{e p}$ decreased significantly. Although gold-standard values are not available for comparison, the values calculated with the PT AIFs are expected to be more accurate than those calculated with the PO AIFs due to the reduced experimental error of data used in the PT AIF. Since the average AUC significantly decreased, it is reasonable to expect the average DCE-MRI parameters to increase. This result can be predicted by observing the pharmacokinetic model used to obtain these parameters (the extended Tofts-Kety model, see Eq. 2.13), assuming a constant tissue $[\mathrm{Gd}]$.

\subsection{Conclusions}

High-quality clinical DCE-MRI requires an accurate AIF measurement to properly characterize tumour perfusion properties. One method to reduce errors in the AIF obtained from the traditional magnitude acquisition is to measure the change in phase induced by the presence of a GBCA as it passes through the tissue following a bolus injection. This method, while better than the SI technique, is sensitive to baseline phase errors which introduce significant uncertainty to the whole AIF. The PT AIF method was 
proposed in Chapter 4 as being superior to the PO method conventionally used. The results presented for HGG patients in this chapter show that the PT AIF is shifted to lower [Gd] values relative to the PO AIF and it is in significantly better agreement with the population averaged AIF published by Parker et al. [67].

The PT AIF method requires that a measurement of $T_{1}\left(\right.$ or $T_{1}^{*}$ ) be added to the protocol. The gold-standard method for measuring $T_{1}$, EPI-IR, cannot be used with patients due to time constraints. In the previous chapter MOLLI was shown to be a good, time efficient substitute. The work presented in this chapter validates that the PT AIF method using MOLLI is an effective, time efficient method for measuring the AIF which is feasible to use with a patient population. This validation holds regardless of which PT AIF method is used.

A comparison of DCE-MRI parameters obtained using the PO AIF with those obtained using the PT AIF shows significant differences in the parameters produced using the extended Tofts-Kety model. This study concludes that, since the DCE-MRI parameters obtained with the PT AIF appear to more reliable, they could improve the diagnostic potential for this population. Investigating this potential would be a logical next step for this research but it is beyond the scope of this thesis. 


\section{Chapter 7}

\section{Conclusions}

A tumour has a unique vascular infrastructure that is formed during a period of rapid growth. These vessels are highly permeable, leading to significantly different perfusion properties than for the surrounding healthy tissue. This difference in perfusion can be observed during clinical imaging, especially with DCE-MRI, where characteristic changes to image contrast occur as an injected gadolinium-based contrast agent (GBCA) passes through the tissue of interest. Quantitative parameters that describe the tumour perfusion can be obtained if the MRI signal change in a nearby feeding artery is also measured. These signal changes can be converted to concentration values for the GBCA ([Gd]) as a function of time as the bolus of contrast agent passes through the artery. This function is known as the Arterial Input Function (AIF). The measurement of the AIF can be performed by observing the change in either the MR signal intensity (SI) or the phase ( $\phi)$ due to changes in [Gd] as the tracer moves through the imaged slices. Unfortunately, both of these methods are prone to significant errors when performed clinically. While the change in $\mathrm{SI}$ is the more commonly performed measurement, the change in $\phi$ to compute 
the AIF (i.e. the "Phase-Only (PO) AIF) offers several advantages over the SI method, one of which is improved accuracy. These advantages include a linear relationship between $\phi$ and [Gd] and a good sensitivity to [Gd] changes over a wide range of [Gd] values (i.e. it does not saturate), including the moderately high concentrations that occur during the acquisition of the AIF.

When an AIF is acquired for a study involving brain tumours a cerebral artery is usually chosen as the location for the measurement. Due to their small diameters, the use of these arteries is particularly susceptible to experimental error from partial volume averaging (PVA) due to contamination of the MR signal by contributions from surrounding tissues. One method to reduce this error is to use a large vein, such as the superior sagittal sinus (SSS), to measure the [Gd] after the GBCA leaves the tumour volume. This function, known as the venous output function (VOF), has many similarities to the AIF and is often substituted for the AIF. In fact, the measured VOF is often a better representation of the true AIF than the measured AIF as a result of PVA which is unavoidable with small arteries. In this thesis, VOF measurements were performed and then treated as if they were AIFs. Consequently, these functions are referred to as AlFs throughout the thesis.

The effect of PVA on the signal measured from the SSS will be much smaller than with a cerebral artery, but it typically will still be present. PVA is only a problem with AIF or VOF measurements if the extravascular signal is different from the intravascular signal; however, this is typically the case for AIF and VOF measurements. When the signal phase is measured, the most important factors that cause the intravascular and extravascular phases to differ were identified to be: (i) the difference in magnetic susceptibility between 
the inside and outside of the vessel, (ii) the motion of the nuclear spins in the blood during the slice selection process, and (iii) the presence of a GBCA inside the vessel. These sources of error were studied theoretically and were simulated in vivo AIF acquisitions using realistic, clinical parameters. It was found that these errors are additive and result in a systematic overestimation of the PO AlF. This effect was determined to be SIdependent, where the phase error was largest for the AIF baseline (i.e. when $[\mathrm{Gd}]=0$ ), smallest at the AIF peak, and a value in between for the AIF washout (i.e. [Gd] equilibrium at the end of the AIF). These phase errors were predicted to be approximately $9^{\circ}, 3^{\circ}$, and $1^{\circ}$ at the baseline, washout, and peak, respectively. The large error in the baseline is significant, since the AIF is computed by subtracting the baseline phase values from each data point to get the final PO AIF curve.

To minimize the effects of this phase overestimation, a novel method to compute the AIF was proposed. Instead of using the baseline phase value to compute the AIF, it can be computed based on phase changes relative to the washout. This method requires independent knowledge of the [Gd] for the AIF washout. For the work presented here, this was obtained using fast and accurate $T_{1}$ measurements for the baseline $\left(T_{1,0}\right)$ and washout $\left(T_{1, w}\right)$ of the AIF curve which were used to compute the true [Gd] of the washout. This new technique, termed the "Phase and $\mathrm{T}_{1}$ " (PT) AIF, was created by first computing the PO AIF, subtracting off the washout [Gd] value from the whole PO AIF and then adding to each point the $[G d]$ value computed from careful $T_{1,0}$ and $T_{1, w}$ measurements. While $T_{1}$ measurements have been shown to be an accurate method for computing [Gd], their long acquisition times prevent them from being used to acquire the entire AIF. 
The PO and PT AIF methods were simulated and compared with a populationaveraged AIF obtained from a large clinical study in the literature. The effects of PVA were explicitly included in these simulations. As anticipated, the PO AIF was higher than the AIF from the literature due to the influence of PVA. The novel PT AIF method was then applied to the PO AIF data and, while it did not reproduce the original, errorless AIF, it did produce an AIF that resembled the literature AIF much more closely.

These simulations promisingly demonstrated that the PT AIF method could be used to reduce the effects of PVA. This methodology was then tested on two types of plastic tubing test objects, or phantoms. The accuracy of the PT AIF method is dependent on the accuracy of measured $T_{1}$ data, so the first set of phantoms was created to test a clinically-practical $T_{1}$ measurement method. These phantoms held non-flowing (static) water with various [Gd] levels that covered the entire range of [Gd] values expected in a clinical AIF acquisition. $T_{1}$ data were also acquired using a "gold-standard" technique (EPI$I R)$, and while it is a highly accurate method, it is very time-consuming ( $6.5 \mathrm{~min})$, and is not used clinically. A time-efficient $T_{1}$ sequence (MOLLI), which was originally developed for cardiac acquisitions, was adapted to acquire $T_{1}$ data for the brain. Two sets of MOLLI sequence parameters were developed to optimally acquire $T_{1}$ data at different [Gd] values: $\mathrm{MOLLI}_{0}$ for the baseline and $\mathrm{MOLLI}_{1}$ for the washout.

It was found that $M O L L I_{0} T_{1}^{*}$ (i.e. apparent $T_{1}$ ) values agreed well with EPI-IR $T_{1}$ data in the low $[\mathrm{Gd}]$ range $([\mathrm{Gd}] \leq 0.26 \mathrm{mM})$ and $\mathrm{MOLL}_{1} \mathrm{~T}_{1}^{*}$ data showed no statistically significant difference from EPI-IR $\mathrm{T}_{1}$ data for the rest of the $[\mathrm{Gd}]$ range. This proved that the faster MOLLI acquisition matched very well with the gold-standard $T_{1}$ measurement 
technique. The $\mathrm{MOLLI}_{0}$ and $\mathrm{MOLLI}_{1} \mathrm{~T}_{1}^{*}$ data were then combined into a single data set $\left(\mathrm{MOLLI}_{\text {comb }}\right)$ to form a calibration curve to allow for the [Gd] computation of an object once that object's $T_{1}$ value is acquired. The slope of this calibration curve is the longitudinal relaxivity, $r_{1}$, and, for the static phantom data, the measured $r_{1}$ agreed well with the literature value (5.1\% error) for the specific GBCA and medium temperature used in this work.

A flowing-water, or dynamic, phantom was then used in which both the PO AIF and $\mathrm{T}_{1}^{*}$ data were acquired. The phantom system was designed to resemble a clinical scan for a patient with a brain tumour, where the input tubing modelled the SSS. $T_{1}$ data were acquired both before and after the injection of the GBCA. While EPI-IR could not be acquired clinically, it could be used with the phantom which did not have the same time constraints. The $T_{1}$ values measured with MOLLI were found to strongly correlate with the EPI-IR $T_{1}$ values $(r=0.995, P<0.001)$ while the $[G d]$ computed from the phase had no correlation with the $[\mathrm{Gd}]$ obtained from the EPI-IR $T_{1}$ data $(r=0.067, P=0.898)$. The PT AIF method was then applied, and similar to the simulation results, the PT AIF was lower in magnitude than the PO AIF. This difference, on average, was $0.24 \mathrm{mM}$ corresponding to a phase error of $12^{\circ}$, which was similar to the theoretical $6^{\circ}$ from the simulations.

Finally, this methodology was performed for a clinical study of patients with highgrade gliomas. The clinical PO and PT AIF acquisitions resembled the data obtained from both simulations and phantoms. This difference in AIF values produced significantly different tumour perfusion parameters. As the PT AIF is predicted to be more accurate, it follows that these newly acquired perfusion parameters could provide better tumour 
diagnoses and prognoses.

DCE-MRI has been shown to be a valuable tool in the diagnosis of various tumour types. The accuracy of DCE-MRI is based on the accuracy of the AIF, but the measurement is prone to significant errors. This thesis has presented a novel phase and $T_{1}$ AIF method that is clinically-feasible was predicted by a theoretical analysis and corresponding simulations to be more accurate than current methods. Experimental results from test objects were in agreement with these theoretical predictions. DCE-MRI parameters obtained with the new method are statistically different from those obtained using the conventional phase-based approach. This strongly suggests that the new parameters are more accurate; however, since the true values for these parameters are not available this could not be tested directly. The use of the DCE-MRI parameters obtained with this new approach for determining the diagnosis and prognosis in patients suspected of having brain cancer seems promising. 


\section{List of References}

[1] Canadian Cancer Society's Advisory Committee on Cancer Statistics. Canadian Cancer Statistics 2017. Canadian Cancer Society, Toronto, ON (2017).

[2] Canadian Cancer Statistics Advisory Committee. Canadian Cancer Statistics 2018. Canadian Cancer Society, Toronto, ON (2018).

[3] N. Majtenyi, H. Gabrani-Juma, R. Klein, R. A. deKemp, G. O. Cron, T. B. Nguyen, and I. G. Cameron. "Comparing arterial input function measurements in DCE-MRI using MOLLI and phase." Medical Physics 43(6), 3644 (2016).

[4] N. Majtenyi, H. Gabrani-Juma, R. Klein, R. A. deKemp, G. O. Cron, T. B. Nguyen, and I. G. Cameron. "Comparison of input function measurements from DCE and MOLLI." Medical Physics 43(8), 4952 (2016).

[5] N. Majtenyi, G. O. Cron, Hanif Gabrani-Juma, A. Greiser, R. A. deKemp, R. Klein, T. B. Nguyen, and I. G. Cameron. "Improved arterial input function measurements using phase-versus-time and modified Look-Locker inversion recovery: Phantom validation study." Proceedings of International Society of Magnetic Resonance of Medicine 25(1), 1903 (2017).

[6] A. Del Sole, A. Falini, L. Ravasi, L. Ottobrini, D. De Marchis, E. Bombardieri, and G. Lucignani. "Anatomical and biochemical investigation of primary brain tumours." European Journal of Nuclear Medicine 28(12), 1851-1872 (2001).

[7] M. Westphal and K. Lamszus. "Circulating biomarkers for gliomas." Nature Reviews Neurology 11(10), 556-566 (2015).

[8] S. K. Joshi and R. Zuniga. High grade glioma - standard approach, obstacles and future directions. In L. R. Morgan (Ed.), Tumors of the Central Nervous System: Primary and Secondary. InTech, Rijeka, pp. 3-29 (2014).

[9] D. N. Louis, A. Perry, G. Reifenberger, A. von Deimling, D. Figarella-Branger, W. K. Cavenee, H. Ohgaki, O. D. Wiestler, P. Kleihues, and D. W. Ellison. "The 2016 World Health Organization classification of tumors of the central nervous system: A summary." Acta Neuropathologica 131(6), 803-820 (2016). 
[10] Q. T. Ostrom, L. Bauchet, F. G. Davis, I. Deltour, J. L. Fisher, C. E. Langer, M. Pekmezci, J. A. Schwartzbaum, M. C. Turner, K. M. Walsh, M. R. Wrensch, and J. S. BarnholtzSloan. "The epidemiology of glioma in adults: A 'state of the science' review." Neuro-Oncology 16(7), 896-913 (2014).

[11] S. M. Afify and M. Seno. "Conversion of stem cells to cancer stem cells: undercurrent of cancer initiation." Cancers 11(3), 345-364 (2019).

[12] S. Brem, R. Cotran, and J. Folkman. "Tumor angiogenesis: a quantitative method for histologic grading." Journal of the National Cancer Institute 48(2), 347-356 (1972).

[13] R. D. Folkerth. "Descriptive analysis and quantification of angiogenesis in human brain tumors." Journal of Neuro-Oncology 50(1), 165-172 (2000).

[14] J. Folkman. "Angiogenesis: An organizing principle for drug discovery?" Nature Reviews Drug Discovery 6(4), 273-286 (2007).

[15] J. C. Anderson, B. C. McFarland, and C. L. Gladson. "New molecular targets in the angiogenic vessels of glioblastoma tumours." Expert Reviews in Molecular Medicine 10(e23), 1-33 (2009).

[16] T. Demuth and M. E. Berens. "Molecular mechanisms of glioma cell migration and invasion." Journal of Neuro-Oncology 70(2), 217-228 (2004).

[17] J. A. Nagy, L. Benjamin, H. Zeng, A. M. Dvorak, and H. F. Dvorak. "Vascular permeability, vascular hyperpermeability and angiogenesis." Angiogenesis 11(2), 109-119 (2008).

[18] S. S. Kety. "The theory and applications of the exchange of inert gas at the lungs and tissues." Pharmacological Reviews 3(1), 1-41 (1951).

[19] J. L. Evelhoch. Tracer measurements of blood flow. In R. Gillies (Ed.), NMR in Physiology and Biomedicine. Academic Press, San Diego, CA, pp. 209-220 (1994).

[20] P. S. Tofts. "Modeling tracer kinetics in dynamic Gd-DTPA MR imaging." Journal of Magnetic Resonance Imaging 7(1), 91-101 (1997).

[21] H. K. Walker, W. D. Hall, and J. W. Hurst. Clinical Methods: The History, Physical, and Laboratory Examinations. Butterworth Publishers, Boston, MA, 3rd edition (1990).

[22] P. S. Tofts, G. Brix, D. L. Buckley, J. L. Evelhoch, E. Henderson, M. V. Knopp, H. B. W. Larsson, T.-Y. Lee, N. A. Mayr, G. J. M. Parker, R. E. Port, J. Taylor, and R. M. Weisskoff. "Estimating kinetic parameters from dynamic contrast-enhanced $\mathrm{T}_{1}$ weighted MRI of a diffusable tracer: Standardized quantities and symbols." Journal of Magnetic Resonance Imaging 10(3), 223-232 (1999).

[23] K. L. Zierler. "Theory of use of indicators to measure blood flow and extracellular volume and calculation of transcapillary movement of tracers." Circulation Research 12(5), 464-471 (1963). 
[24] C. Crone. "The permeability of capillaries in various organs as determined by the use of 'indicator diffusion' method." Acta Physiologica 58(4), 292-305 (1963).

[25] L. Østergaard, R. M. Weisskoff, D. A. Chesler, C. Gyldensted, and B. R. Rosen. "High resolution measurement of cerebral blood flow using intravascular tracer bolus passages. Part I: mathematical approach and statistical analysis." Magnetic Resonance in Medicine 36(5), 715-725 (1996).

[26] M. Bergamino, L. Bonzano, F. Levrero, G. L. Mancardi, and L. Roccatagliata. “A review of technical aspects of $T_{1}$-weighted dynamic contrast-enhanced magnetic resonance imaging (DCE-MRI) in human brain tumors." Physica Medica 30(6), 635643 (2014).

[27] Z. Liang and P. Lauterbur. Principles of Magnetic Resonance Imaging: A Signal Processing Perspective. IEEE Press, Piscataway, NJ (2000).

[28] J. Prince and J. Links. Medical Imaging Signals and Systems. Pearson Prentice-Hall, Upper Saddle River, NJ (2006).

[29] F. Reif. Fundamentals of Statistical and Thermal Physics. Waveland Press, Long Grove, IL (2009).

[30] E. M. Haacke, R. W. Brown, M. R. Thompson, and R. Venkatesan. Magnetic Resonance Imaging: Physical Principles and Sequence Design. Wiley (1999).

[31] F. M. L. Amirouche and T. Jia. "Automatic elimination of the undetermined multipliers in Kane's equations using a Pseudo Uptriangular Decomposition (PUTD) method." Computers \& Structures 27(2), 203-210 (1987).

[32] R. Deichmann and A. Haase. "Quantification of $T_{1}$ Values by SNAPSHOT-FLASH NMR Imaging." Journal of Magnetic Resonance 96(3), 608-612 (1992).

[33] D. C. Look and D. R. Locker. "Time saving in measurement of NMR and EPR relaxation times." Review of Scientific Instruments 41(2), 250-251 (1970).

[34] D. R. Messroghli, A. Radjenovic, S. Kozerke, D. M. Higgins, M. U. Sivananthan, and J. P. Ridgway. "Modified Look-Locker inversion recovery (MOLLI) for high-resolution $\mathrm{T}_{1}$ mapping of the heart." Magnetic Resonance in Medicine 52(1), 141-146 (2004).

[35] A. Oppelt, R. Graumann, H. Barfuß, H. Fischer, W. Hartl, and W. Schajor. "FISP - a new fast MRI sequence." Electromedica 54(1), 15-18 (1986).

[36] K. Scheffler and J. Hennig. " $T_{1}$ quantification with inversion recovery True-FISP." Magnetic Resonance in Medicine 45(4), 720-723 (2001).

[37] E. Henderson, G. McKinnon, T. Y. Lee, and B. K. Rutt. "A fast 3D Look-Locker method for volumetric $T_{1}$ mapping." Magnetic Resonance Imaging 17(8), 1163-1171 (1999). 
[38] J.G. Sled, I. Levesque, A. C. Santos, S. J. Francis, S. Narayanan, S. D. Brass, D. L. Arnold, and G. B. Pike. "Regional variations in normal brain shown by quantitative magnetization transfer imaging." Magnetic Resonance in Medicine 51(2), 299-303 (2004).

[39] J. G. Sled and G. B. Pike. "Quantitative imaging of magnetization transfer exchange and relaxation properties in vivo using MRI." Magnetic Resonance in Medicine 46(5), 923-931 (2001).

[40] I. Levesque, J. G. Sled, S. Narayanan, A. C. Santos, S. D. Brass, S. J. Francis, D. L. Arnold, and G. B. Pike. "The role of edema and demyelination in chronic $T_{1}$ black holes: a quantitative magnetization transfer study." Journal of Magnetic Resonance Imaging 21(2), 103-110 (2005).

[41] N. Stikov, M. Boudreau, I. R. Levesque, C. L. Tardiff, J. K. Barral, and G. B. Pike. "On the accuracy of $T_{1}$ mapping: searching for common ground." Magnetic Resonance in Medicine 73(2), 514-522 (2015).

[42] Y.-D. Xiao, R. Paudel, J. Liu, C. Ma, Z.-S. Zhang, and S.-K. Zhou. “MRI contrast agents: classification and application (Review)." International Journal of Molecular Medicine 38(5), 1319-1326 (2016).

[43] A. F. Stalder, D. v. Elverfeldt, D. Paul, J. Hennig, and M. Markl. "Variable echo time imaging: Signal characteristics of 1-M gadobutrol contrast agent at 1.5 and 3 T." Magnetic Resonance in Medicine 59(1), 113-123 (2008).

[44] J. Pintaske, P. Martirosian, H. Graf, G. Erb, K.-P. Lodemann, C. D. Claussen, and F. Schick. "Relaxivity of gadopentetate dimeglumine (Magnevist), gadobutrol (Gadovist), and gadobenate dimeglumine (MultiHance) in human blood plasma at 0.2, 1.5, and 3 Tesla." Investigative Radiology 41(3), 213-221 (2006).

[45] L. de Rochefort, T. Nguyen, R. Brown, P. Spincemaille, G. Choi, J. Weinsaft, M. R. Prince, and Y. Wang. "In vivo quantification of contrast agent concentration using the induced magnetic field for time-resolved arterial input function measurement with MRI." Medical Physics 35(12), 5328-5339 (2008).

[46] H. J Weinmann, M. Laniado, and W. Mützel. "Pharmacokinetics of GdDTPA/ dimeglumine after intravenous injection into healthy volunteers." Physiological Chemistry and Physics and Medical NMR 16(2), 167-172 (1984).

[47] G. O. Cron, J. C. Wallace, W. D. Stevens, T. Fortin, B. A. Pappas, R. C. Wilkins, F. Kelcz, and G. E. Santyr. "A comparison of $\mathrm{T}_{2}^{*}$-weighted magnitude and phase imaging for measuring the arterial input function in the rat aorta following intravenous injection of gadolinium contrast agent." Magnetic Resonance Imaging 23(5), 619-627 (2005). 
[48] R. Ellinger, C. Kremser, M. F. H. Schocke, C. Kolbitsch, J. Griebel, S. R. Felber, and F. T. Aichner. "The impact of peak saturation of the arterial input function on quantitative evaluation of dynamic susceptibility contrast-enhanced MR studies." Journal of Computer Assisted Tomography 24(6), 942-948 (2000).

[49] F. Calamante. "Arterial input function in perfusion MRI: A comprehensive review." Progress in Nuclear Magnetic Resonance Spectroscopy 74(1), 1-32 (2013).

[50] M. S. Kotys, E. Akbudak, J. Markham, and T. E. Conturo. "Precision, signal-to-noise ratio, and dose optimization of magnitude and phase arterial input functions in dynamic susceptibility contrast MRI." Journal of Magnetic Resonance Imaging 25(3), 598-611 (2007).

[51] M. Y. Zhao, M. Mezue, A. R. Segerdahl, T. W. Okell, I. Tracey, Y. Xiao, and M. A. Chappell. "A systematic study of the sensitivity of partial volume correction methods for the quantification of perfusion from pseudo-continuous arterial spin labeling MRI." Neurolmage 162(1), 384-397 (2017).

[52] M. J. P. van Osch, E. P. A. Vonken, C. J. G. Bakker, and M. A. Viergever. "Correcting partial volume artifacts of the arterial input function in quantitative cerebral perfusion MRI." Magnetic Resonance in Medicine 45(3), 477-485 (2001).

[53] P. Bouillot, B. M. A. Delattre, O. Brina, R. Ouared, M. Farhat, C. Chnafa, D. A. Steinmen, K.-O. Lovblad, V. M. Pereira, and M. I. Vargas. "3D phase contrast MRI: partial volume correction for robust blood flow quantification in small intracranial vessels." Magnetic Resonance in Medicine 79(1), 129-140 (2018).

[54] M. J. P. van Osch, J. van der Grond, and C. J. G. Bakker. "Partial volume effects on arterial input functions: shape and amplitude distortions and their correction." Journal of Magnetic Resonance Imaging 22(6), 704-709 (2005).

[55] J. Krejza, M. Arkuszewski, S. E. Kasner, J. Weigele, A. Ustymowicz, R. W. Hurst, B. L. Cucchiara, and S. R. Messe. "Carotid artery diameter in men and women and the relation to body and neck size." Stroke 37(4), 1103-1105 (2006).

[56] J. M. Serrador, P. A. Picot, B. K. Rutt, J. K. Shoemaker, and R. L. Bondar. "MRI measures of middle cerebral artery diameter in conscious humans during simulated orthostasis." Stroke 31(7), 1672-1678 (2000).

[57] B. T. Andrews, M. Dujovny, H. G. Mirchandani, and J. I. Ausman. "Microsurgical anatomy of the venous drainage into the superior sagittal sinus." Neurosurgery 24(4), 514-520 (1989).

[58] C. Foottit, G. O. Cron, M. J. Hogan, T. B. Nguyen, and I. Cameron. "Determination of the venous output function from MR signal phase: feasibility for quantitative DCEMRI in human brain." Magnetic Resonance in Medicine 63(3), 772-781 (2010). 
[59] V. C. Keil, B. Mädler, J. Gieseke, R. Fimmers, E. Hattingen, H. H. Schild, and D. R. Hadizadeh. "Effects of arterial input function selection on kinetic parameters in brain dynamic contrast-enhanced MRI." Magnetic Resonance Imaging 40(1), 83-90 (2017).

[60] C. Li, M. C. Langham, C. L. Epstein, J. F. Magland, J. Wu, J. Gee, and F. W. Wehrli. "Accuracy of the cylinder approximation for susceptometric measurement of intravascular oxygen saturation." Magnetic Resonance in Medicine 67(3), 808-813 (2012).

[61] F. W. Wehrli and E. M. Haacke. Principles of MR imaging. In J. Potchen, E. M. Haacke, J. E. Siebert, and A. Gottschalk (Eds.), Magnetic Resonance Angiography: Concepts and Applications. Mosby-Year Book, Inc., St. Louis, MO, pp. 9-34 (1993).

[62] R. Weisskoff and S. Kiihne. "MRI susceptometry: image-based measurement of absolute susceptibility of MR contrast agents and human blood." Magnetic Resonance in Medicine 24(2), 375-383 (1992).

[63] W. M. Spees, D. A. Yablonsky, M. C. Oswood, and J. J. H. Ackerman. "Water proton MR properties of human blood at 1.5 Tesla: magnetic susceptibility, $T_{1}, T_{2}, T_{2}^{*}$, and non-Lorentzian signal behavior." Magnetic Resonance in Medicine 45(4), 533-542 (2001).

[64] V. Jain, M. C. Langham, and F. W. Wehrli. "MRI estimation of global brain oxygen consumption rate." Journal of Cerebral Blood Flow \& Metabolism 30(9), 1598-1607 (2010).

[65] J. E. Jordan, N. J. Pelc, and D. R. Enzmann. "Velocity and flow quantitation in the superior sagittal sinus with ungated and cine (gated) phase-contrast MR imaging." Journal of Magnetic Resonance Imaging 4(1), 25-28 (1994).

[66] C. G. Caro, T. J. Pedley, R. C. Schroter, and W. A. Seed. The Mechanics of the Circulation. Cambridge University Press, Cambridge, 2nd edition (2011).

[67] G. J. M. Parker, C. Roberts, A. Macdonald, G. A. Buonaccorsi, S. Cheung, D. L. Buckley, A. Jackson, Y. Watson, K. Davies, and G. C. Jayson. "Experimentally-derived functional form for a population-averaged high-temporal-resolution arterial input function for dynamic contrast-enhanced MRI." Magnetic Resonance in Medicine 56(5), 993-1000 (2006).

[68] L. Georgiou, D. J. Wilson, N. Sharma, T. J. Perren, and D. L. Buckley. "A functional form for a representative individual arterial input function measured from a population using high temporal resolution DCE MRI." Magnetic Resonance in Medicine 81(3), 1955-1963 (2019). 
[69] M. A. Horsfield, J. S. Thorton, A. Gill, H. R. Jager, A. N. Priest, and B. Morgan. “A functional form for injected MRI Gd-chelate contrast agent concentration incorporating recirculation, extravasation and excretion." Physics in Medicine \& Biology 54(9), 2933-2949 (2009).

[70] T. E. Conturo and G. D. Smith. "Signal-to-noise in phase angle reconstruction: dynamic range extension using phase reference offsets." Magnetic Resonance in Medicine 15(3), 420-437 (1990).

[71] H.-L. M. Cheng. "Investigation and optimization of parameter accuracy in dynamic contrast-enhanced MRI." Journal of Magnetic Resonance Imaging 28(3), 736-743 (2008).

[72] G. J. Stanisz, E. E. Odrobina, J. Pun, M. Escaravage, S. J. Graham, M. J. Bronskill, and R. M. Henkelman. " $T_{1}, T_{2}$ relaxation and magnetization transfer in tissue at $3 \mathrm{~T}$." Magnetic Resonance in Medicine 54(3), 507-512 (2005).

[73] H. Gudbjartsson and S. Patz. "The Rician distribution of noisy MRI data." Magnetic Resonance in Medicine 34(6), 910-914 (1995).

[74] X. Li, R. A. Priest, W. J. Woodward, F. Siddiqui, T. M. Beer, M. G. Garzotto, W. D. Rooney, and C. S. Springer Jr. "Cell membrane water exchange effects in prostate DCE-MRI." Journal of Magnetic Resonance 218(1), 77-85 (2012).

[75] I. van der Schaaf, E.-J. Vonken, A. Waaijer, B. Velthuis, M. Quist, and T. van Osch. "Influence of partial volume on venous output and arterial input function." American Journal of Neuroradiology 27(1), 46-50 (2006).

[76] M. C. Schabel and D. L. Parker. "Uncertainty and bias in contrast concentration measurements using spoiled gradient echo pulse sequences." Physics in Medicine \& Biology 53(9), 2345-2373 (2008).

[77] J. G. Korporaal, C. A. T. van den Berg, M. J. P. van Osch, G. Groenendaal, M. van Vulpen, and U. A. van der Heide. "Phase-based arterial input function measurements in the femoral arteries for quantification of dynamic contrastenhanced (DCE) MRI and comparison with DCE-CT." Magnetic Resonance in Medicine 66(5), 1267-1274 (2011).

[78] G. O. Cron, C. Foottit, T. E. Yankeelov, L. I. Avruch, M. E. Schweitzer, and I. Cameron. "Arterial input functions determined from MR signal magnitude and phase for quantitative dynamic contrast-enhanced MRI in the human pelvis." Magnetic Resonance in Medicine 66(2), 498-504 (2011).

[79] F. F. J. Simonis, A. Sbrizzi, E. Beld, J. J. W. Lagendijk, and C. A. T. van den Berg. "Improving the arterial input function in dynamic contrast enhanced MRI by fitting the signal in the complex plane." Magnetic Resonance in Medicine 76(4), 1236-1245 (2016). 
[80] P. Brynolfsson, J. Yu, R. Wirestam, M. Karlsson, and A. Garpebring. "Combining phase and magnitude information for contrast agent quantification in dynamic contrast-enhanced MRI using statistical modeling." Magnetic Resonance in Medicine 74(4), 1156-1164 (2015).

[81] G. Cowan. Statistical Data Analysis. Oxford University Press Inc., New York, NY (1998).

[82] B. Driscoll, H. Keller, and C. Coolens. "Development of a dynamic flow imaging phantom for dynamic contrast-enhanced CT." Medical Physics 38(8), 4866-4880 (2011).

[83] H. Gabrani-Juma, O. J. Clark, A. Pourmoghaddas, B. Driscoll, R. G. Wells, R. A. deKemp, and R. Klein. "Validation of a multimodality flow phantom and its application for assessment of dynamic SPECT and PET technologies." IEEE Transactions on Medical Imaging 36(1), 132-141 (2017).

[84] S. Nekolla, T. Gneiting, J. Syha, R. Deichmann, and A. Haase. "T$T_{1}$ maps by k-space reduced snapshot-FLASH MRI." Journal of Compute Assisted Tomography 16(2), 327-332 (1972).

[85] X. Zhang, E. T. Petersen, E. Ghariq, J. B. De Vis, A. G. Webb, W. M. Teeuwisse, J. Hendikse, and M. J. P. van Osch. "In vivo blood T 1 measurements at 1.5 T, 3 T, 7 T." Magnetic Resonance in Medicine 70(4), 1082-1086 (2013).

[86] T. B. Nguyen, G. O. Cron, J. F. Mercier, C. Foottit, C. H. Torres, S. Chakraborty, J. Woulfe, G. H. Jansen, J. M. Caudrelier, J. Sinclair, M. J. Hogan, R. E. Thornhill, and I. G. Cameron. "Diagnostic accuracy of dynamic contrast-enhanced MR imaging using a phase-derived vascular input function in the preoperative grading of gliomas." American Journal of Neuroradiology 33(8), 1539-1545 (2012).

[87] A. Garpebring, R. Wirestam, N Östlund, and M. Karlsson. "Effects of inflow and radiofrequency spoiling on the arterial input function in dynamic contrast-enhanced MRI: a combined phantom and simulation study." Magnetic Resonance in Medicine 65(6), 1670-1679 (2011).

[88] H.-L. M. Cheng. " $T_{1}$ measurement of flowing blood and arterial input function determination for quantitative 3D $\mathrm{T}_{1}$-weighted DCE-MRI." Journal of Magnetic Resonance Imaging 25(5), 1073-1078 (2007).

[89] R. Morabito, C. Alafaci, S. Pergolizzi, A. Pontoriero, G. lati, L. Bonanno, M. Gaeta, F. M. Salpietro, and E. Mormina. "DCE and DSC perfusion MRI diagnostic accuracy in the follow-up of primary and metastatic intra-axial brain tumors treated by radiosurgery with Cyberknife." Radiation Oncology 14(65), 1-9 (2019). 
[90] X. Li, Y. Cai, B. Moloney, Y. Chen, W. Huang, M. Woods, F. V. Coakley, W. D. Rooney, M. G. Garzotto, and C. S. Springer Jr. "Relative sensitivities of DCE-MRI pharmacokinetic parameters to arterial input function (AIF) scaling." Journal of Magnetic Resonance 269(1), 104-112 (2016).

[91] A. Garpebring, P. Brynolfsson, J. Yu, R. Wirestam, A. Johansson, T. Asklund, and M. Karlsson. "Uncertainty estimation in dynamic contrast-enhanced MRI." Magnetic Resonance in Medicine 69(4), 992-1002 (2013).

[92] C. Lavini. "Simulating the effect of input errors on the accuracy of Tofts' pharmacokinetic model parameters." Magnetic Resonance Imaging 33(2), 222-235 (2015). 